\title{
Discovery and Biosynthesis of Natural Products from New Zealand Soil
} Metagenome Libraries

\author{
By
}

\section{Luke Jordan Stevenson}

A thesis submitted to the Victoria University of Wellington in fulfilment of the requirements for the degree of Doctor of Philosophy In Biotechnology

Victoria University of Wellington (2020) 



\begin{abstract}
Antibiotic discovery rates dramatically declined following the "golden age" of the 1940's to the 1960's. The platforms that underpinned that age of discovery rested upon laboratory cultivation of a small clade of bacteria, the actinomycetes, primarily isolated from soil environments. Fermentation extracts of these isolated bacteria have provided the majority of antibiotics and anticancer small molecules still used today. By applying modern genetic analysis techniques to these same environmental sources that have previously yielded such success, we can uncover new biosynthetic pathways, and bioactive compounds. The work described in this thesis investigated New Zealand soil metagenomes for this purpose.

Four large metagenome libraries were constructed from the microbiomes of diverse soil environments. These were then interrogated by a functional screening approach in a knockout Escherichia coli strain, to recover a large collection of the biosynthetic gene clusters responsible for bacterial secondary metabolite production. Using different modes of bioinformatic analysis, these gene clusters were demonstrated to have both phylogenetic divergence, and functional difference from bacterial biosynthesis pathways previously discovered from culture based studies.

Two additional biosynthetic pathways were recovered from one of these metagenome libraries, and in each case found to have novel genetic features. These gene clusters were further studied by heterologous expression within Streptomyces albus production hosts. One of these gene clusters produced small aromatic polyketide compounds, the structure of one of which was solved by chemical analytic techniques, and found to be a new chemical entity.

The second gene cluster was demonstrated to have similarity to known aureolic acid biosynthesis gene clusters - a class of potent anticancer natural products. Heterologous expression resulted in the production of many metabolites, two of which were characterised and found to be new members of this chemical class.

The research in this thesis both validates the use of metagenomic analysis for future natural product discovery efforts, and adds to a growing body of evidence that understudied clades of bacteria have an untapped biosynthetic potential that can be accessed by metagenomic methods.
\end{abstract}




\section{Acknowledgements}

I would like to thank my primary supervisor, Dr Jeremy Owen. Thank you for your support and advice, and the encouraging words when I needed them. My favourite words of wisdom: "If you really think about what it is we're trying to do, it's kind of amazing that it ever really works at all." Keep those grants coming. Also thanks to my secondary supervisor, Prof David Ackerley. Thank you for inviting me into your lab way back when, and for encouraging me to take on a $\mathrm{PhD}$ in the first place. Working with the two of you has been a privilege, and I feel extraordinarily fortunate to have learned from you both.

Thank you to the Maurice Wilkins Centre and Victoria University of Wellington for supporting me throughout my $\mathrm{PhD}$ studies. I would like to extend additional thanks to the University, for providing the supportive environment for learning, and to the technicians who keep everything running.

To my friends and lab mates, thank you for making this such an amazing place to work. In particular, thank you to the members of the MetaMax team: Matt, Mark, Liwei, Vincent, Izzie, Diana, Manu, Peng, Max, and Chanel. Starting up a new lab group can be a bit of journey, but I think we've made something pretty great. Also thanks to the Ackerley lab group: Elsie, Katherine, Becky, Alistair, Michelle, Rory, Abby, Kelsi, Jasmine, Mitch, Hannah, Vincent, Sarah, Janine, and Jenni. Sharing a space with you all has been a lot of fun and I really do appreciate everything you've taught me.

A special thanks to the chemists, Joe and Rob, for all of your assistance along the way, and for just being such kind and generous people.

Thank you to my family and friends who have helped me throughout this entire journey. 


\section{Contents}

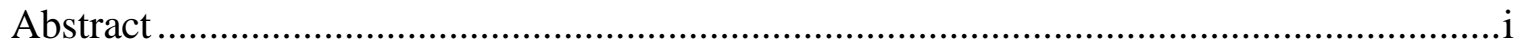

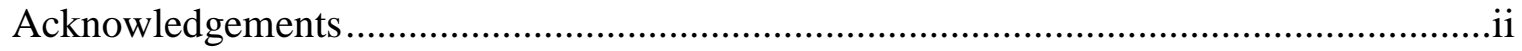

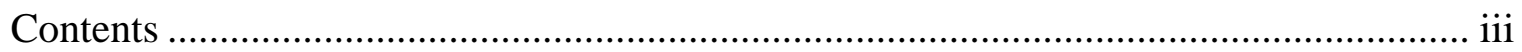

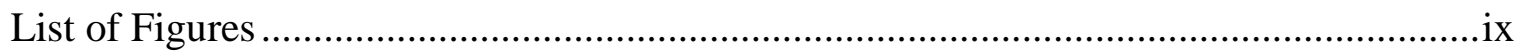

List of Tables ......................................................................................................

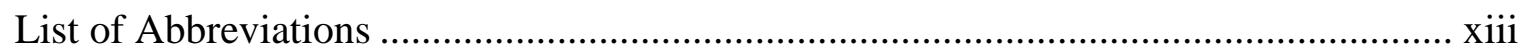

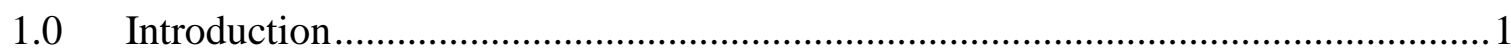

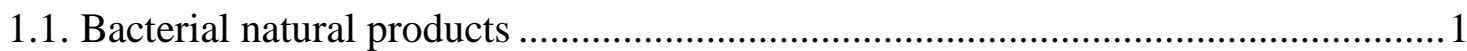

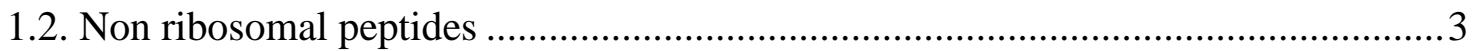

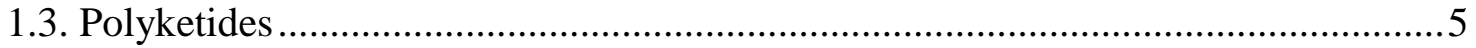

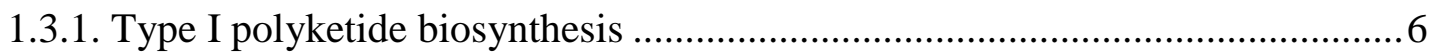

1.3.2. Type II polyketide biosynthesis ..............................................................

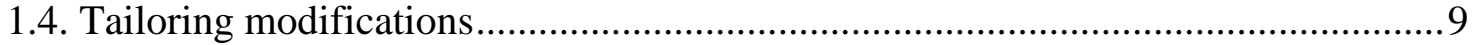

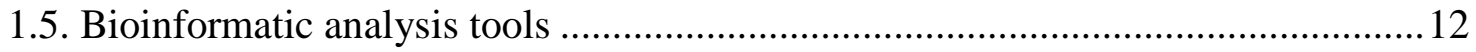

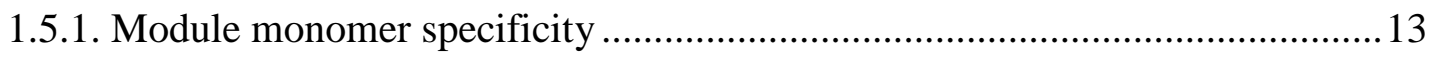

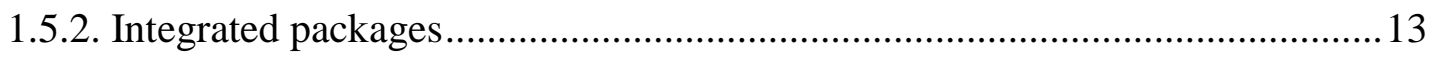

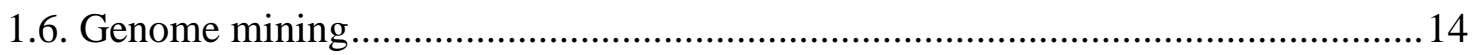

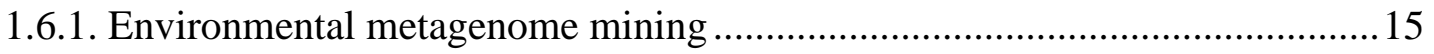

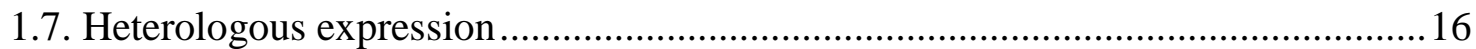

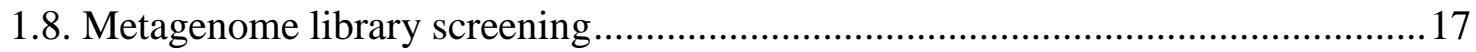

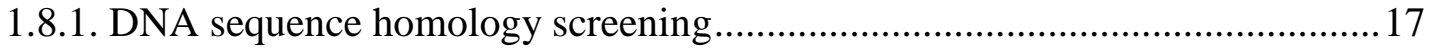

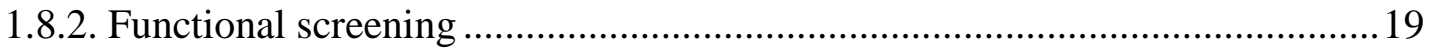

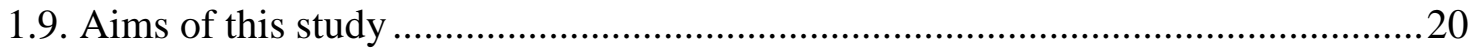

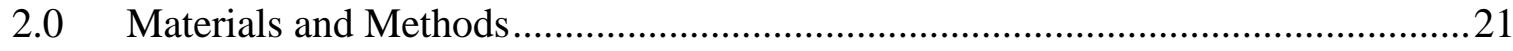

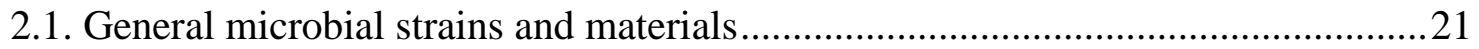


2.1.1. Bacterial and yeast strains

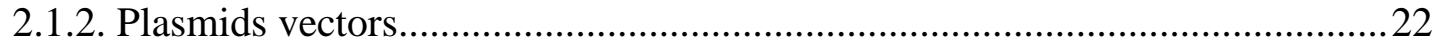

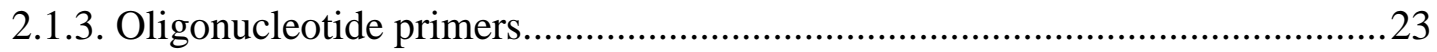

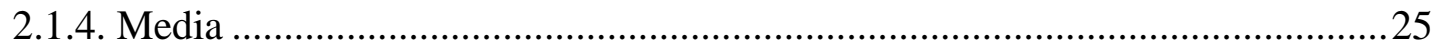

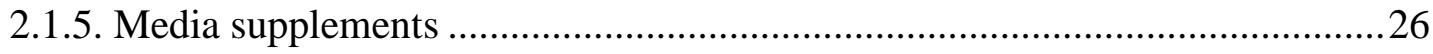

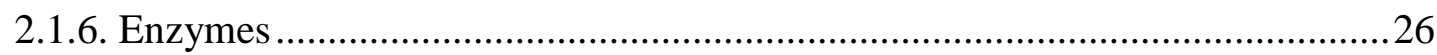

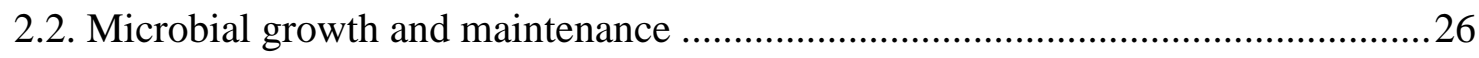

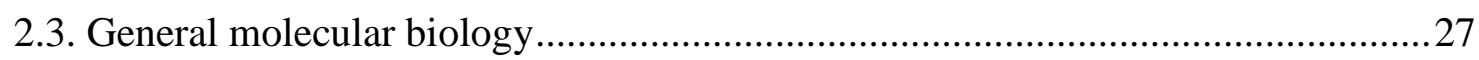

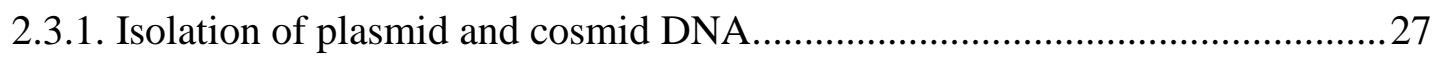

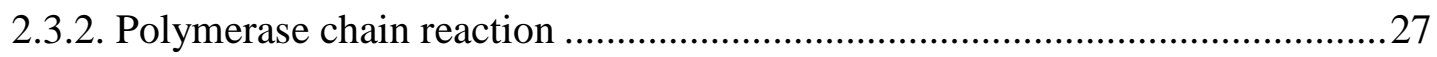

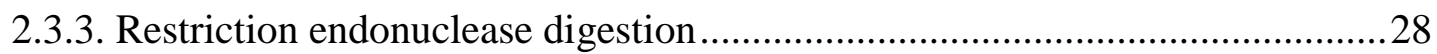

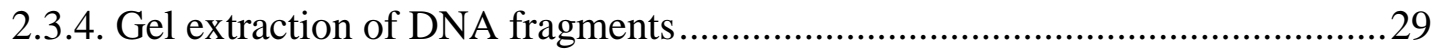

2.3.5. Preparation and transformation of competent cells ......................................29

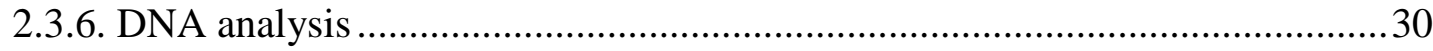

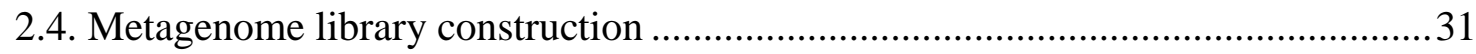

2.4.1. Environmental DNA extractions from soil ................................................... 31

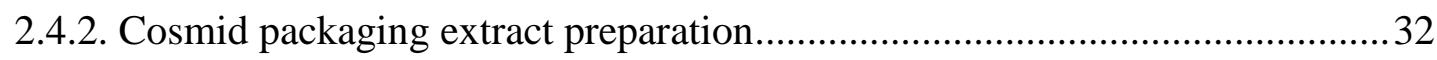

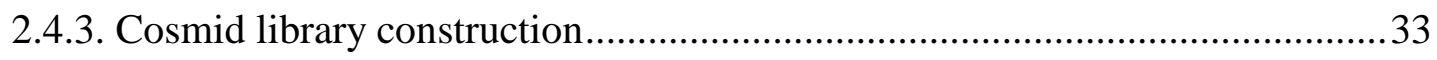

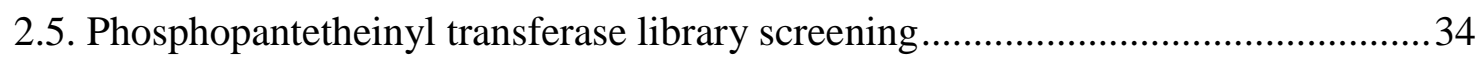

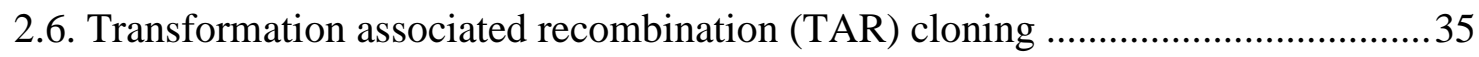

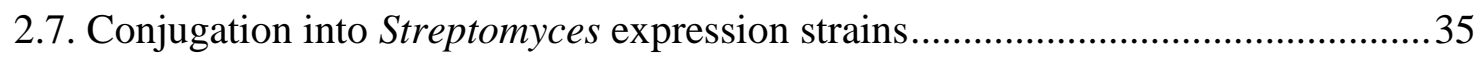

2.8. Heterologous expression of biosynthetic gene clusters ..........................................36

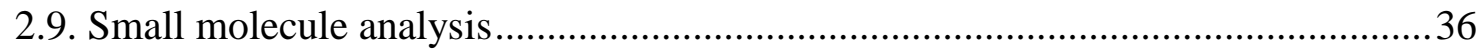

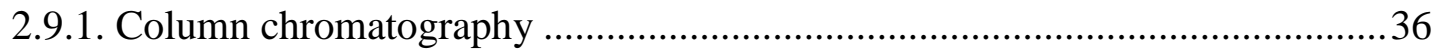

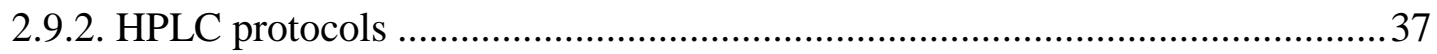

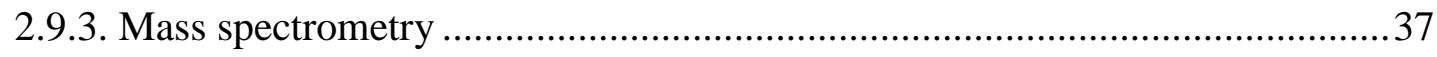

2.9.4. Nuclear Magnetic Resonance spectroscopy....................................................38 


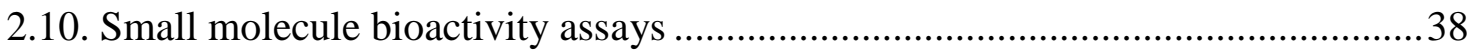

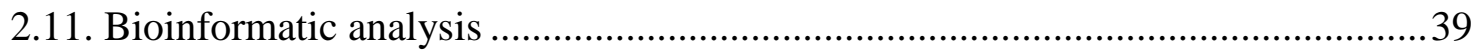

2.11.1. Assembly of next generation sequencing data............................................ 39

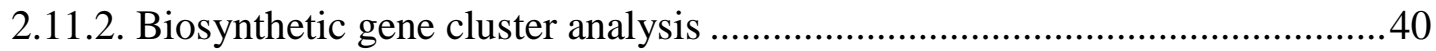

3.0 Soil environmental DNA extraction and metagenome library construction........... 43

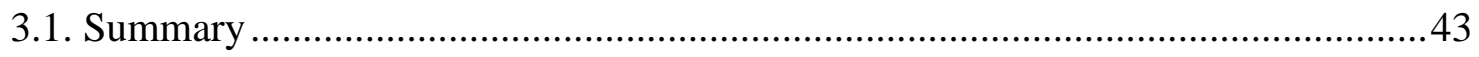

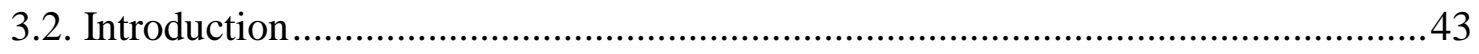

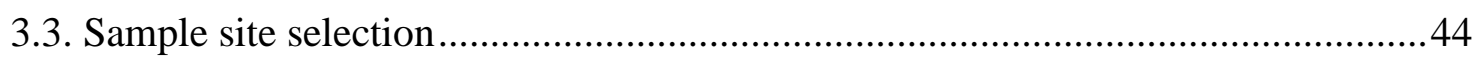

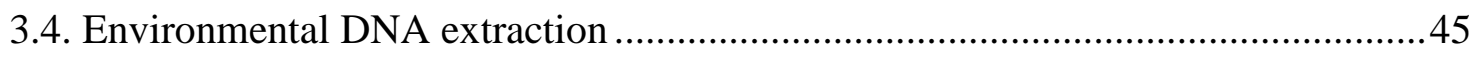

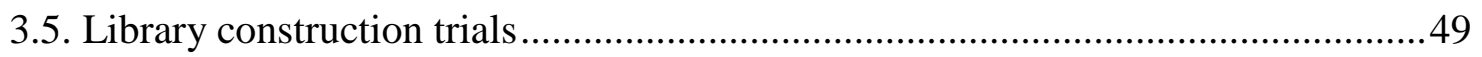

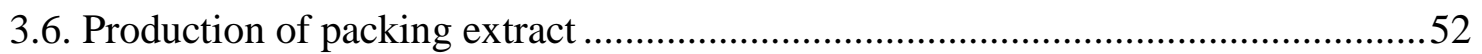

3.7. Construction of four soil metagenome cosmid libraries .....................................54

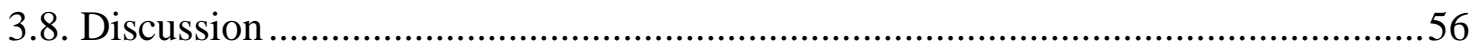

4.0 Functional screening of soil metagenome libraries and bioinformatic analysis .....59

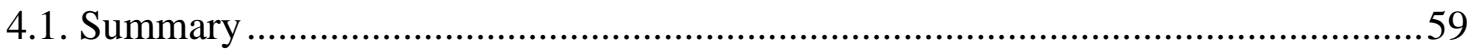

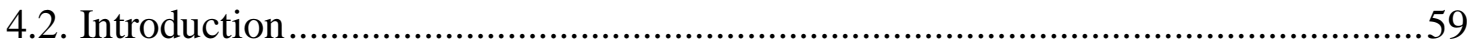

4.3. Small scale screening of the Castle Hill metagenome library ..............................61

4.4. Large scale screening and sequencing of the remaining three soil metagenome

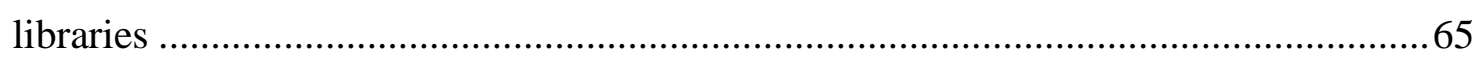

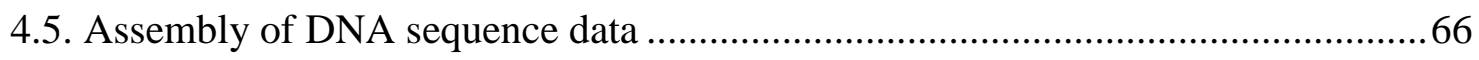

4.6. Gene level diversity in metagenome biosynthetic gene clusters .........................69

4.6.1. Taxonomic diversity of metagenome biosynthetic gene cluster genes............69

4.6.2. Functional diversity of metagenome biosynthetic gene cluster genes ..............73

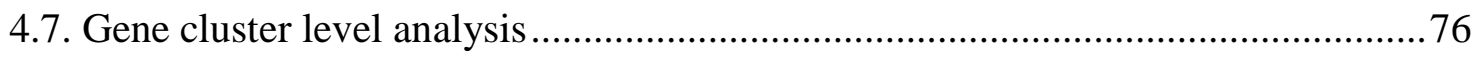

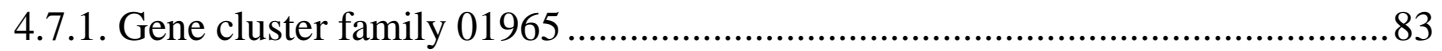

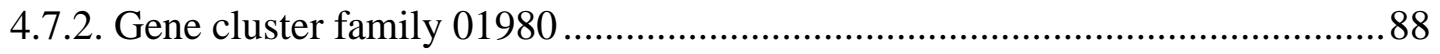

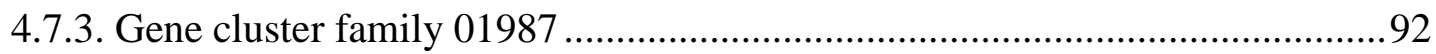


5.0 Type II polyketide biosynthetic gene cluster recovery and metabolite characterisation

5.1. Summary 101

5.2. $\mathrm{KS}_{\alpha} \mathrm{PCR}$ screening 102

5.3. Initial expression studies 103

5.4. Fermentation and compound isolation 107

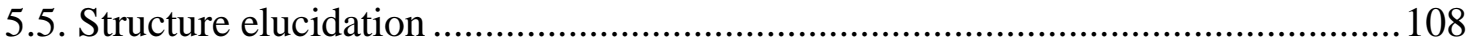

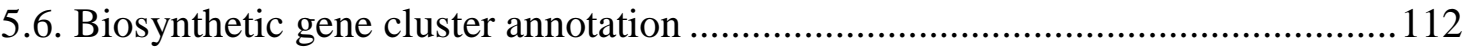

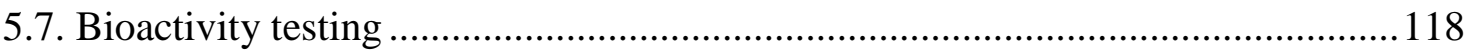

5.8. Discussion

6.0 Discovery and reconstitution of novel aureolic acid biosynthesis gene cluster and characterisation of major metabolites

6.1. Summary

6.2. Introduction 122

6.3. Reconstitution of the biosynthetic gene cluster 126

6.4. Aureolic acid gene cluster annotation 131

6.5. Heterologous expression studies 136

6.6. Isolation and structural elucidation of compound 4 143

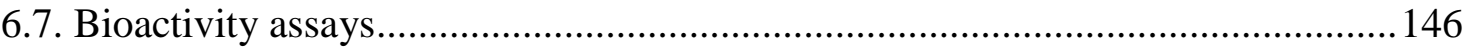

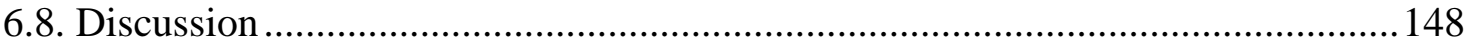

7.0 Summary, conclusions and future directions ............................................... 151

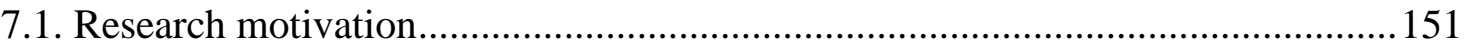

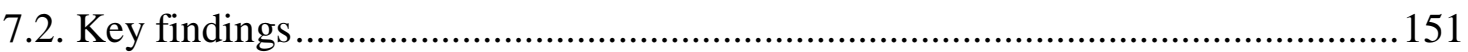

7.2.1. Sequence first biosynthetic survey........................................................... 151

7.2.2. Gene cluster and metabolites of the D9 cosmid........................................... 152

7.2.3. Novel aureolic acid gene cluster and metabolites........................................153

7.3. Future directions 
7.3.1. Biosynthetic gene clusters from functional screening .....

7.3.2. D9 cluster metabolites

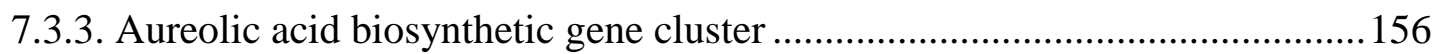

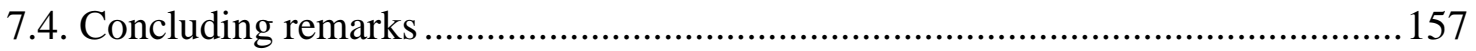

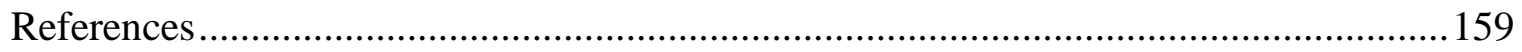

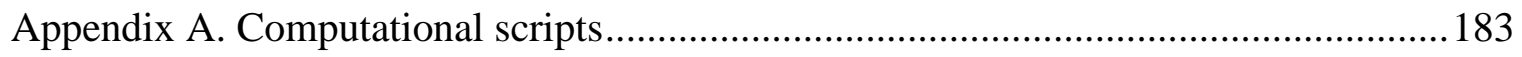

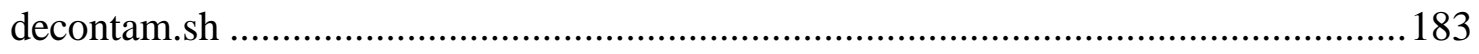

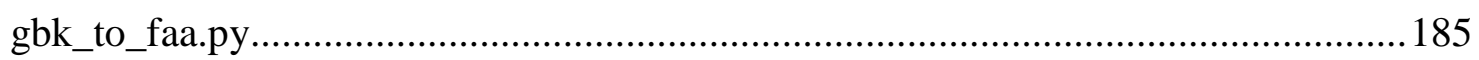

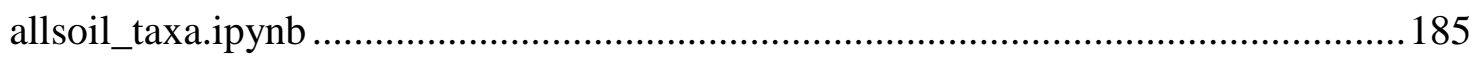

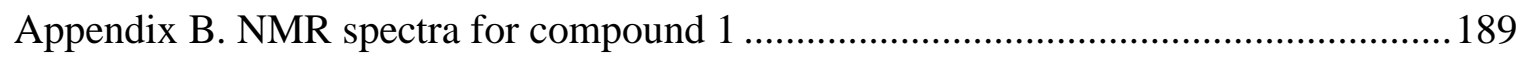

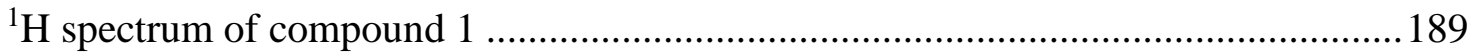

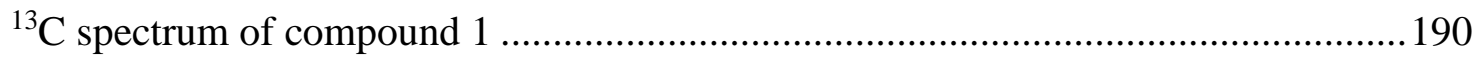

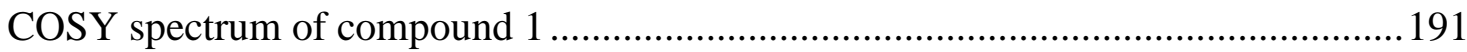

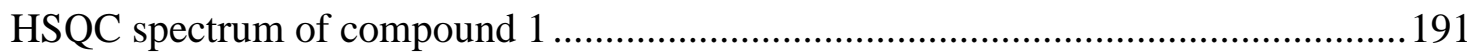

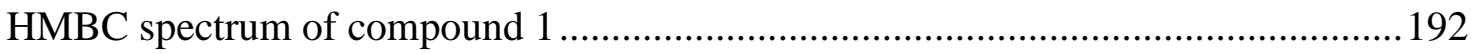

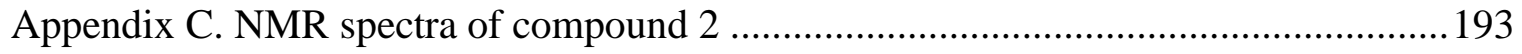

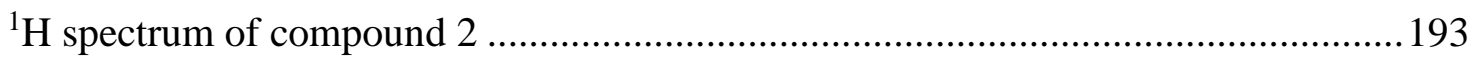

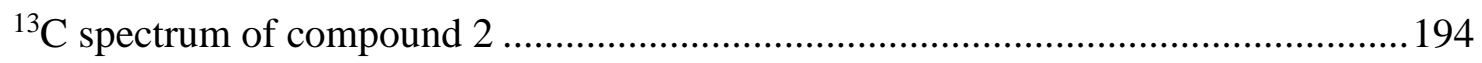

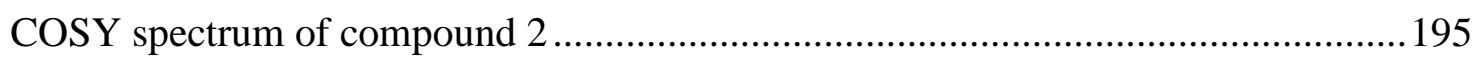

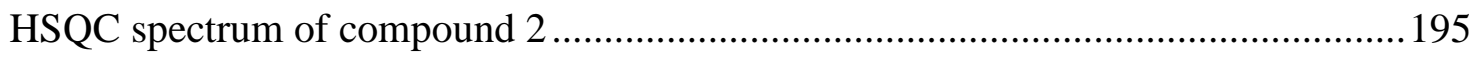

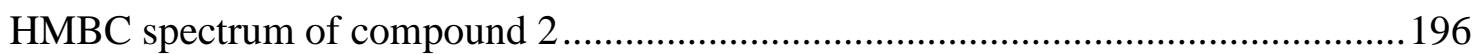

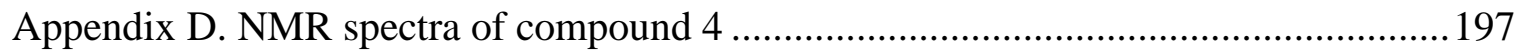

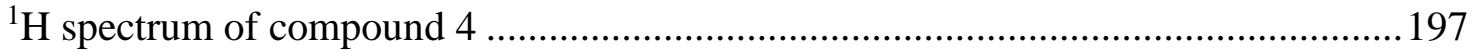

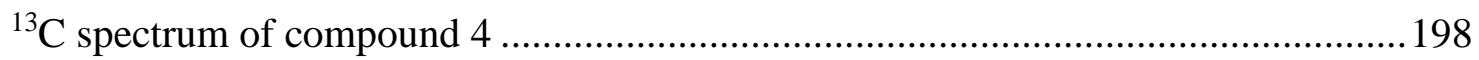

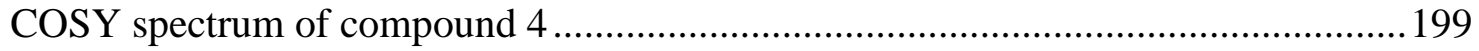

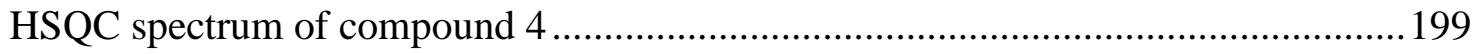

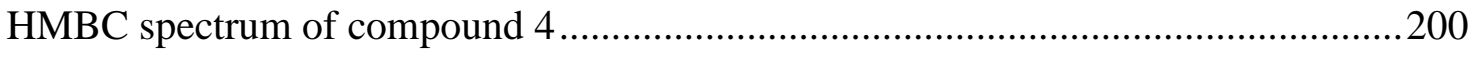


HSQC-TOCSY spectrum of compound 4

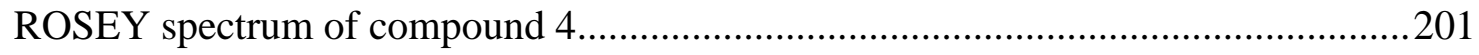

Appendix E. Extended MS/MS spectra of compounds 4, 5, and mithramycin ...............202

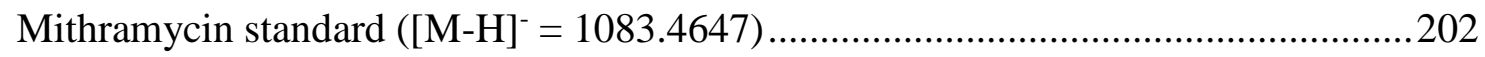

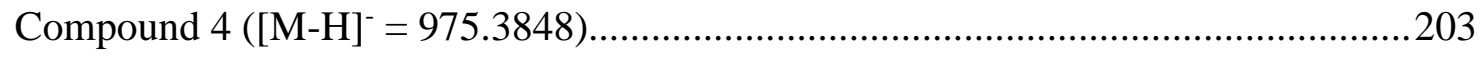

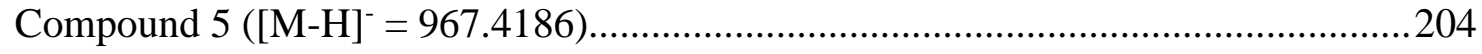




\section{List of Figures}

Figure 1.1 - Generic workflow for bacterial natural product discovery from environmental samples using a DNA sequence first approach.......................................................... 3

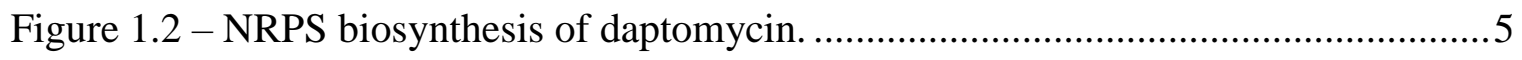

Figure 1.3 - Type I polyketide biosynthesis exemplified by epothilone............................

Figure 1.4 - A depiction of the minimal type II PKS biosynthesis machinery, and examples

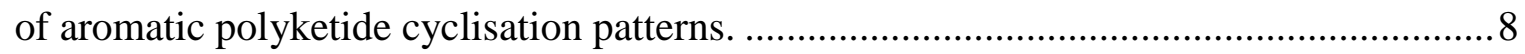

Figure 1.5 - Tailoring enzyme modifications to the aureolic acid chromomycin $\mathrm{A}_{3} \ldots \ldots . .10$

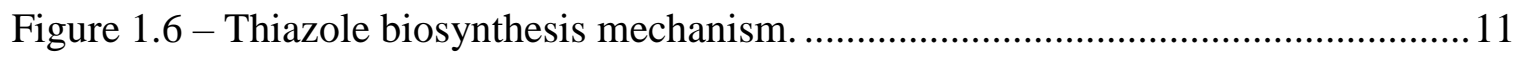

Figure 1.7 - TE-domain mediated macrocyclisation resulting in peptidolactone and

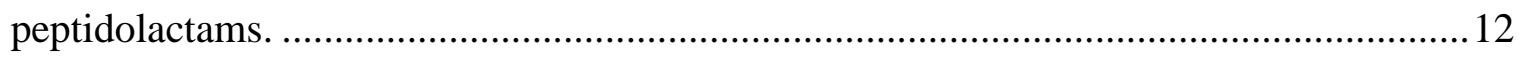

Figure 3.1 - Soil sample collection sites and soil classification map of New Zealand.... 45

Figure 3.2 - Environmental DNA extraction and grading process.................................46

Figure 3.3 - Extracts and agarose gel of environmental DNA extractions from soils 1-13.

Figure 3.4 - Agarose gel of environmental DNA extractions from soils 14-55.............. 48

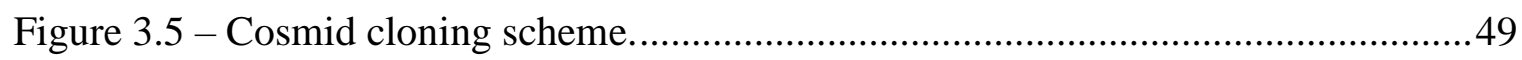

Figure 3.6 - Map of soil sample collection sites around the South Island of New Zealand.

Figure 3.7 - Restriction analysis of twelve constructed cosmids. ...................................54

Figure 3.8 - Arrayed metagenome library from Half Moon Bay soil eDNA....................55

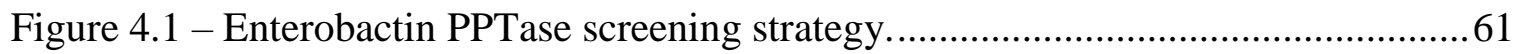

Figure 4.2 - Taxonomic distribution of open reading frames in metagenomic biosynthetic

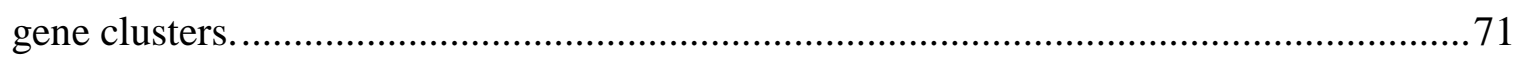

Figure 4.3 - Metagenome biosynthetic gene cluster amino acid identity plots.................75

Figure 4.4 - Biosynthetic gene cluster analysis pipeline............................................... 78

Figure 4.5 - BiG-SCAPE network diagram of all metagenome and MIBiG biosynthetic

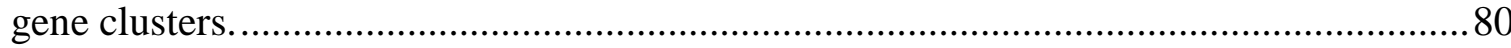

Figure 4.6 - Taxonomic classification of genes in gene cluster families 01965, 01980, 01987.

Figure 4.7 - Gene cluster family 01965 (GCF_01965). .83 
Figure 4.8 - Adenylation domain monomer selectivity for each biosynthetic gene cluster in GCF_01965.

Figure 4.9 - Speculative biosynthesis scheme for generic GCF_01965 biosynthesis.......86

Figure 4.10 - Gene cluster family 01980 (GCF_01980).

Figure 4.11 - Known compounds produced by biosynthetic gene clusters with homology to those in GCF_01980.

Figure 4.12 - Gene cluster family 01987 (GCF_01987).

Figure 4.13 - Known compounds produced by biosynthetic gene clusters with homology to those in GCF_01987.

Figure 4.14 - Speculative biosynthesis scheme for RX_70, a representative member of GCF_01987. .95

Figure 5.1 - Type II polyketide biosynthesis degenerate primer screening. 103

Figure 5.2 - HPLC trace of S. albus D9 culture extract. 104

Figure 5.3 - Key NMR correlations by COSY and HMBC for compound 1.

Figure 5.4 - Comparison of chemical structures of compound 1 and setomimycin. 112

Figure 5.5 - Metagenome polyketide biosynthetic gene cluster compared to the setomimycin biosynthetic gene cluster.

Figure 5.6 - Possible biosynthesis scheme for compound 1.

Figure 6.1 - Chemical structures of the aureolic acid family of compounds.

Figure 6.2 - Number of publications in SciFinder Scholar that make reference to aureolic acid, mithramycin or chromomycin by year up to 2018 .

Figure 6.3 - Condensed biosynthesis scheme for aureolic acids mithramycin and chromomycin $\mathrm{A}_{3}$.

Figure 6.4 - Generic cosmid overlap PCR recovery process.

Figure 6.5 - Transformation associated recombination scheme for reassembling the metagenomic aureolic acid biosynthetic gene cluster.

Figure 6.6 - Aureolic acid biosynthetic gene cluster annotation 132

Figure 6.7 - Biosynthetic pathways for production of deoxy sugars in aureolic acid biosynthesis.

Figure 6.8 - Expression of complete D3_2 metagenome aureolic acid pathway.

Figure 6.9 - MS/MS spectra of a mithramycin standard, compound 4 and compound 5.

Figure 6.10 - Constitutive expression of ring OIV_W cassette for production of "ring opened" compound 5. 
Figure 6.11 - Key NMR correlations by HMBC and COSY for the structure elucidation of the compound 4 aglycone.

Figure 6.12 - Key NMR correlations and coupling relationships for determination of saccharide chain order in compound 4.

Figure 6.13 - Structural comparison of compound 4 and 5 to the most closely related aureolic acids. 146 


\section{List of Tables}

Table 2.1 - Bacterial and yeast strains used in this study...........................................22

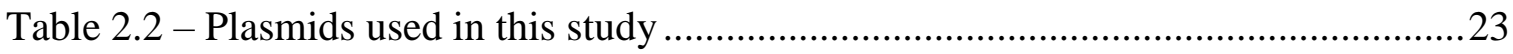

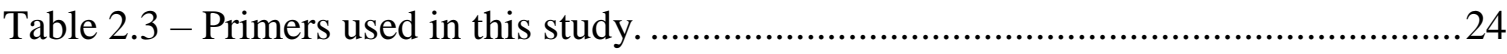

Table 2.4 - Standard Biomix Red PCR components and cycle protocol. .........................28

Table 2.5 - Standard Q5 High-Fidelity PCR components and cycle protocol. .................28

Table 3.1 - Library construction trial results for pWEB::tnc libraries. ..............................51

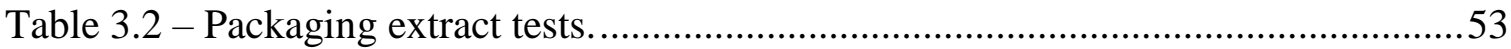

Table 3.3 - Cosmid metagenome libraries constructed in this study. ..............................56

Table 4.1 - End-sequencing of cosmids from $\Delta e n t D$ screened $\mathrm{CH}$ library......................65

Table 4.2 - Summary table of sequence data assemblies and biosynthetic gene cluster

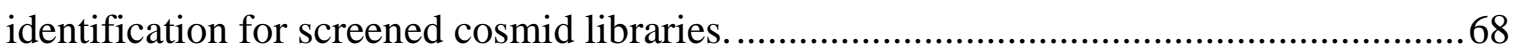

Table 4.3 - Phylum distribution table of open reading frames from metagenome sequences, and $\mathrm{MIBiG}$ and NCBI databases.

Table 4.4 - Metagenome library biosynthetic gene cluster amino acid sequence identities to nearest homologue $\mathrm{MIBiG}$ reference database.

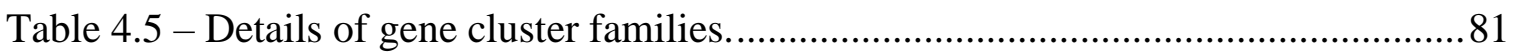

Table 4.6 - Amino acid biosynthesis genes present in GCF_01965...............................85

Table 5.1 - Metagenome D9 pathway metabolite extraction methods.............................106

Table $5.2-{ }^{1} \mathrm{H}$ and ${ }^{13} \mathrm{C}$ NMR data for compound 1 in deuterated chloroform ( $\delta$ in ppm).

Table 5.3 - Metagenome polyketide biosynthetic gene cluster gene annotations and alignment of homologous proteins from the setomimycin biosynthetic gene cluster. ....115

Table 5.4 - Bioactivity assay results of antimicrobial and cytotoxicity assays...............119

Table 6.1 - Aureolic acid biosynthetic gene cluster gene assignment table.

Table 6.2 - Preliminary zone of inhibition screening of crude culture extracts against three test strains 138

Table 6.3 - Bioactivity assay results of antimicrobial and cytotoxicity assays. 147 


\section{List of Abbreviations}

1D

2D

aa

ACP

A-domain

AI

$\operatorname{Amp}^{R}$

antiSMASH

$\mathrm{Apr}^{\mathrm{R}}$

AT-domain

BAC

BGC

BiG-SCAPE

BLAST

BLASTp

BLASTx

bp

C-domain

CH

$\mathrm{Chl}^{\mathrm{R}}$

CoA

CORASON

COSY

Cy-domain

DAD

$\operatorname{ddH}_{2} \mathrm{O}$

DH-domain

DMSO

DNA

DSS

eDNA

E-domain

EDTA

EIC

ER-domain

gDNA one dimensional

two dimensional

amino acid

acyl carrier protein

adenylation domain

adjacency index

ampicillin resistance

antibiotics and secondary metabolite analysis shell

apramycin resistance

acyltransferase domain

bacterial artificial chromosome

biosynthetic gene cluster

biosynthetic gene similarity clustering and prospecting engine

basic local alignment search tool

protein BLAST

nucleotide 6 frame translation protein BLAST

base pair

condensation domain

Castle Hill Basin (library)

chloramphenicol resistance

coenzyme A

core analysis of syntenic orthologs to prioritize natural productbiosynthetic gene clusters

${ }^{1} \mathrm{H}{ }^{1} \mathrm{H}$ correlation spectroscopy

heterocyclisation domain

diode array detector

double distilled $\mathrm{H}_{2} \mathrm{O}$

dehydratase domain

dimethyl sulfoxide

deoxyribonucleic acid

domain sequence similarity

environmental DNA

epimerisation domain

ethylenediaminetetraacetic acid

extracted ion chromatograph

enoyl reductase domain

genomic DNA 


$\begin{array}{ll}\text { GCF } & \text { gene cluster family } \\ \text { HM } & \text { Half Moon Bay (library) } \\ \text { HMBC } & { }^{1} \mathrm{H}^{13} \text { C heteronuclear multiple bond connectivity } \\ \text { HMM } & \text { hidden Markov model } \\ \text { HPLC } & \text { High-performance liquid chromatography } \\ \text { HR-MS } & \text { high resolution mass spectrometry } \\ \text { HSQC } & { }^{1} \mathrm{H}^{13} \text { C heteronuclear single quantum coherence } \\ \text { Hyg } & \text { hygromycin resistance } \\ \text { IC50 } & \text { half maximal inhibitory concentration } \\ \text { JI } & \text { Jaccard index } \\ \text { Kan } & \text { kanamycin resistance } \\ \text { kb } & \text { kilo base } \\ \text { KR-domain } & \text { ketoreductase domain } \\ \text { KS-domain } & \text { ketosynthase domain } \\ \text { KS } \boldsymbol{\alpha} & \text { ketosynthase alpha } \\ \text { KS } & \text { ketosynthase beta } \\ \text { LB } & \text { lysogeny broth } \\ \text { LCMS } & \text { liquid chromatography-mass spectrometry } \\ \text { MIBiG } & \text { minimum information about a biosynthetic gene cluster } \\ \text { MIC } & \text { minimum inhibitory concentration } \\ \text { MRSA } & \text { methicillin-resistant Staphylococcus aureus } \\ \text { MS/MS } & \text { tandem mass spectrometry } \\ \text { MT-domain } & \text { methyltransferase domain } \\ \text { NCBI } & \text { National Center for Biotechnology Information } \\ \text { NCBI-nr } & \text { NCBI non-redundant protein sequences database } \\ \text { NGS } & \text { next-generation sequencing } \\ \text { NMR } & \text { nuclear magnetic resonance } \\ \text { NP } & \text { natural product } \\ \text { NRP } & \text { non-ribosomal peptide } \\ \text { NRPS } & \text { non-ribosomal peptide synthetase } \\ \text { nt } & \text { nucleotide } \\ \text { OD } 600 & \text { optical density at 600 nm } \\ \text { ORF } & \text { open reading frame } \\ \text { Ox-domain } & \text { oxidation domain } \\ \text { PCR } & \text { polymerase chain reaction } \\ \text { PDR } & \text { pleotropic drug resistance } \\ \text { PK } & \text { polyketide } \\ \text { PKS } & \text { polyketide synthase } \\ \text { PPTase } & \text { reliventrifugal force } \\ \text { RU } & \end{array}$


RX

SDS

SL

SPAdes

TAE

TAR

taxID

TBE

T-domain

TE

TE-domain

Tris

TSB

$\mathbf{v} / \mathbf{v}$

$\mathbf{w} / \mathbf{v}$

VRE

YPD
Roxburgh (library)

sodium dodecyl sulfate

Saint Anne's Lagoon (library)

St. Petersburg genome assembler

tris/acetate/EDTA buffer

transformation associated recombination

taxonomic ID

tris/borate/EDTA buffer

thiolation domain

tris/EDTA buffer

thioesterase domain

tris(hydroxymethyl)aminomethane

tryptic soy broth

volume for volume

weight for volume

vancomycin-resistant Enterococcus faecalis

yeast peptone dextrose medium 


\subsection{Introduction}

\subsection{Bacterial natural products}

With the rise of antibiotic resistance, and amid growing fears of food insecurity in a post climate change world, interest in natural products has grown ${ }^{1,2}$. However, the 1940s to 1960s golden era of antibiotic discovery has been followed by a long "dark age", with few examples of new antibiotic class discovery in recent decades ${ }^{2,3}$. The golden era was underpinned by the Waksman platform, whereby microbes (specifically actinomycetes) derived from environmental soil samples were cultured on specific laboratory growth media, and culture extracts assayed for bioactivity ${ }^{4,5}$. This platform eventually fell down because of high rates of rediscovery, making the process unproductive and ultimately commercially non-viable ${ }^{3,6}$. This outcome has been attributed in large part to the low proportion of bacterial species in nature that can be cultured in a laboratory environment ${ }^{7-}$ ${ }^{10}$, and that few secondary metabolite pathways that will be activated under those growth conditions ${ }^{11-13}$.

There is a growing movement to return to the systematic screening of environmental bacteria as per the successful Waksman platform of the golden era, but this time applying advances in genomics and metabolic understanding ${ }^{3-5}$. In particular, molecular phylogenetic studies have validated the "plate count anomaly", providing conclusive data to support the long-held suspicion that the true microbial diversity of soil is 2-3 orders of magnitude greater than that routinely accessed in lab culture conditions ${ }^{7-10}$. Genetic interrogation of non-cultivated microbes from environmental samples (via metagenomics) is beginning to provide access to the previously inaccessible biosynthetic capacity of the bacterial majority, and leading to discovery of previously unknown drug-like natural products ${ }^{14-16}$. Natural products have a very good track record in clinic, with $73 \%$ of approved antibacterial small molecules, and $83 \%$ of small molecule anticancer drugs being either natural products, or derivatives ${ }^{2}$.

In the search for new bacterial secondary metabolites, this thesis will focus on nonribosomal peptides (NRPs) and polyketides (PKs), two important classes of natural product that span a wide range of functional properties, including antibiotics (daptomycin, erythromycin), anticancer agents (bleomycin, epothilone), and immunomodulators 
(cyclosporine A, rapamycin). NRPs and PKs are unique in terms of the understanding we have gained over the logic that underpins their biosynthesis by large (mega-)synth(et)ase enzymes ${ }^{17-20}$. Non-ribosomal peptide synthetases (NRPSs) and polyketide synthases (PKSs) are usually genetically encoded in biosynthetic gene clusters, many of which have been well studied and characterised ${ }^{21}$. This extensive characterisation has revealed rules of biosynthetic logic that make it possible to predict the core structure of the metabolite product by examining the nucleotide sequence of the synthetase genes within the gene cluster $^{22,23}$. Additionally, in a process termed retrobiosynthesis, one can work in reverse to predict the order of synthetase units likely to be encoded by a biosynthetic gene cluster to yield a particular metabolite ${ }^{24,25}$. It is this ready tractability for movement of information from genetic code to chemical metabolite, and back, that makes natural product discovery via mining of bacterial genomic sequences so appealing. Within genomic sequencing data, many NRPS and PKS biosynthetic gene clusters have been discovered by genome mining efforts, including entirely new pathways from species that had previously been well characterised 11,26-29. Even so, the sources for this natural product diversity are fundamentally the same as those used for the Waksman protocol, i.e. cultivated microbes. By applying these new discovery tools creatively to the "microbial dark matter" that is becoming accessible in metagenomes, the potential for discovery of chemical novelty is far greater.

In this study, cosmid DNA libraries from soil metagenomes were constructed, and interrogated for the bacterial secondary metabolism they contained. In this way, we sought to apply modern molecular biology techniques to traditional sources, to discover the hidden genetic and chemical diversity present in New Zealand soil bacteria. 


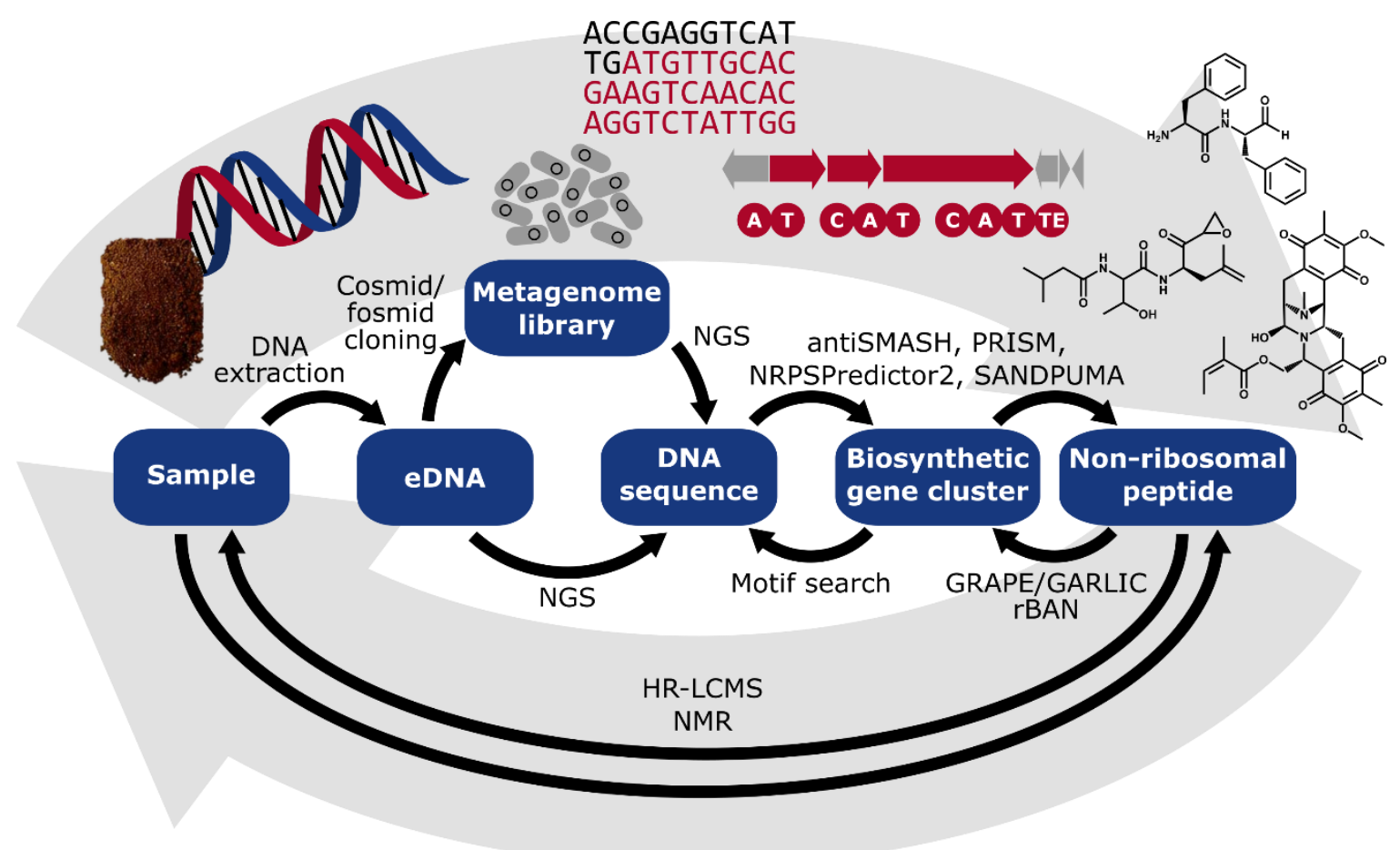

Figure 1.1 - Generic workflow for bacterial natural product discovery from environmental samples using a DNA sequence first approach. In this workflow, the environmental DNA (eDNA) is extracted from a soil sample, and is either directly sequenced, or first cloned into a metagenome library prior to next generation sequencing (NGS). In this DNA sequence data biosynthetic gene clusters can be identified using a variety of bioinformatic programs. Expression of these gene clusters or detection within environmental samples can lead to the discovery of new metabolites (e.g. non-ribosomal peptides). Reproduced from Stevenson et al. $2019^{30}$.

\subsection{Non ribosomal peptides}

The central model for NRP biosynthesis is a linear assembly line (Figure 1.2), wherein modular units in the assembly line catalyse the addition of each amino acid monomer to the growing peptide chain ${ }^{19}$. Each amino acid monomer is selected by an adenylation (A) domain that exhibits specificity for a particular substrate, which may be a proteinogenic amino acid, or one of over 500 variant monomers that have been reported to be accepted by NRPS assembly lines ${ }^{31}$. Downstream of each A-domain is a carrier protein/thiolation (T-) domain, which is modified with a 4' phosphopantetheine prosthetic group, a flexible "arm" that shuttles intermediates within and between the various catalytic domains of the modules in the assembly line. This key modification is catalysed by a phosphopantetheinyl transferase (PPTase) enzyme, which derives the 4' phosphopantetheine group from coenzyme A and tethers it to the T-domain of each NRPS module. Post-activation (by adenylation) of the substrate selected at the A-domain, it is reacted with the sulfhydryl 
group on the free end of the 4' phosphopantetheine arm. Amide bond formation can then be catalysed by condensation (C-) domains, which are typically located at the $\mathrm{N}$-terminal end of every module other than the "initiation module" at the start of the assembly line. Peptide synthesis begins when the "donor" substrate activated by the initiation module is received by the $\mathrm{C}$-domain associated with the second module in the assembly line and condensed with the "acceptor" substrate activated by the A-domain of that module. The growing peptide chain is then passed down the assembly line in this fashion, through multiple "elongation" modules, before arriving at a final "termination module". There, subsequent to formation of the final amide bond, the T-tethered peptide is presented to the terminal domain to be hydrolysed from the phosphopantetheinyl arm. A thioesterase (TE-) domain, terminal reductase (R-) domain, or a terminal C-domain may catalyse this release step, in some cases causing macrocyclisation of the peptide. Additional structural diversity in the NRP can be introduced either during synthesis, by the action of embedded tailoring domains such as methyltransferase (MT-), oxidation (Ox-), heterocyclisation (Cy-), or epimerisation (E-) domains, or post-synthesis modifications by cytochrome $\mathrm{P} 450$ enzymes ( $\beta$-hydroxylation, or oxidative cyclisation of amino acid sidechains), and glycosyltransferase enzymes ${ }^{32,33}$. NRPS pathways can be encoded as a single gene, producing a single enzyme comprised of multiple modules, but in bacterial systems are far more commonly found as a series of genes each encoding one or more modules, or even independent domains ${ }^{34,35}$. Genes are normally arranged according to the "co-linearity rule", such that the linear order of genetic modules within a biosynthetic gene cluster reflects the ultimate order of monomers in the assembled peptide ${ }^{34,35}$. Some NRPS gene clusters encode $m b t H$ homologues, which can bind to A-domains in NRP biosynthesis to increase activity, or alter specificity ${ }^{36}$. 


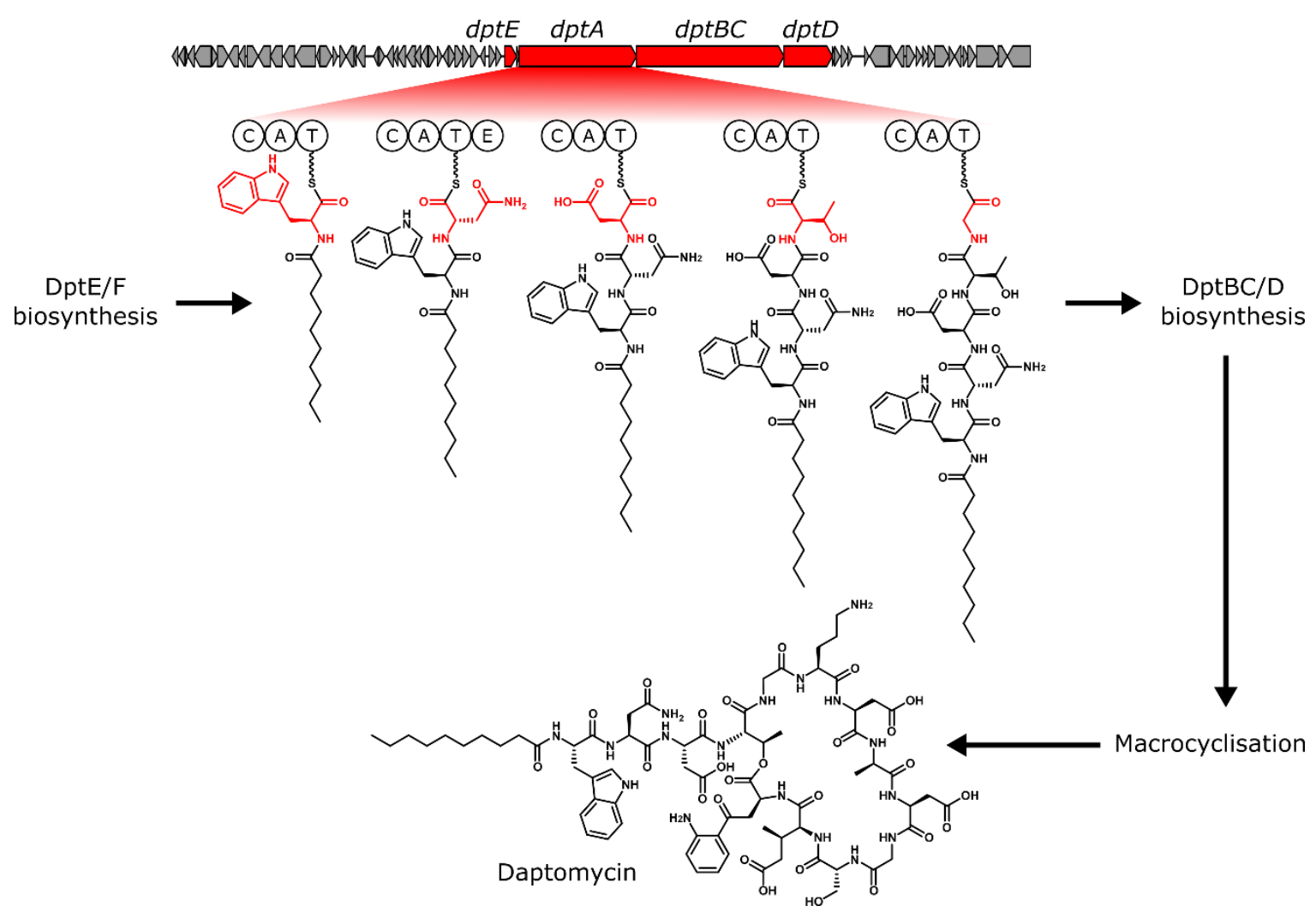

Figure 1.2 - NRPS biosynthesis of daptomycin. The biosynthetic gene cluster of daptomycin is depicted above, with the NRPS genes coloured red (dptA-E). The five modules of the NRPS enzyme DptA are then depicted, with their amino acid contribution to the growing non-ribosomal peptide chain coloured red at each step. The module A-domain specificities result in the addition of L-tryptophan, D-aspartate (due to the action of the module's E-domain), L-aspartic acid, L-threonine, and glycine.

\subsection{Polyketides}

Analogous to NRPS systems, PKS pathways assemble acyl-coenzyme A monomers into polyketide natural products ${ }^{18,37,38}$. There are three broad classifications of PKS, based on the mechanisms used to assemble the monomers. Type I PKSs are multi-enzyme pathways, with catalytic domains covalently linked (in cis) and arranged in an assembly line much like NRPSs. Type II PKSs consist of mono-functional enzymes, acting iteratively in trans to assemble multiple monomers into the PK scaffold. Type III enzymes are more common in plant species, and use acyl-CoA derived monomers directly in synthesis, without first tethering them to the phosphopantetheinylated T-domain.

Only type I and II PKS biosynthesis feature in this thesis, and their functions are discussed further below. 


\subsubsection{Type I polyketide biosynthesis}

A minimal type I PKS module consists of an acyltransferase (AT-) domain, which loads specific ketide extender units to the phosphopantetheinyl arm of the PPTase-activated Tdomain, in a manner analogous to the interaction of NRPS A- and T-domains (Figure 1.3). Ketosynthase (KS-) domains catalyse the extension of the growing PK chain by the extender unit monomer by a decarboxylative condensation reaction ${ }^{37}$. After condensation of the substrates, the newly incorporated carbonyl group may be modified, increasing the available carbon backbone diversity at each module ${ }^{18}$. Ketoreductase (KR-) domains reduce the ketone carbonyl to a hydroxyl, dehydratase ( $\mathrm{DH}-$ ) domains dehydrate across the beta carbon to remove the hydroxyl and reduce to an alkene, and enoyl reductase (ER-) domains further reduces to a single carbon-carbon bond.

Bacterial type I PKSs are arranged into modules, where each module in the assembly-line pathway extends the growing PK product by one ketide unit. Iterative type I PKS pathways have also been described in bacteria, however these are more common in fungi. Some bacterial type I PKS pathways do not have AT-domains that are covalently linked in the biosynthesis module (cis), instead the AT-domains are separately transcribed as independent enzymes that act in trans. These pathways are described as TransAT-PKS biosynthesis pathways ${ }^{39}$.

In addition to the successive reduction events which may occur within each module, methyl branching can be a source of PK chemical diversity. This can be a function of the selectivity of the AT-domain of the module for a methylmalonyl-CoA substrate rather than malonylCoA, resulting in a methylated extension to the growing PK chain ${ }^{40}$. Alternatively, as in NRPS biosynthesis, dedicated MT-domains may be present within a module to methylate the incoming extender unit ${ }^{41}$.

Type I PKS pathways may produce linear polyketides, or macrocyclic polyketides (macrolides) when hydrolytic release from the biosynthetic machinery is coupled to a cyclisation event. 


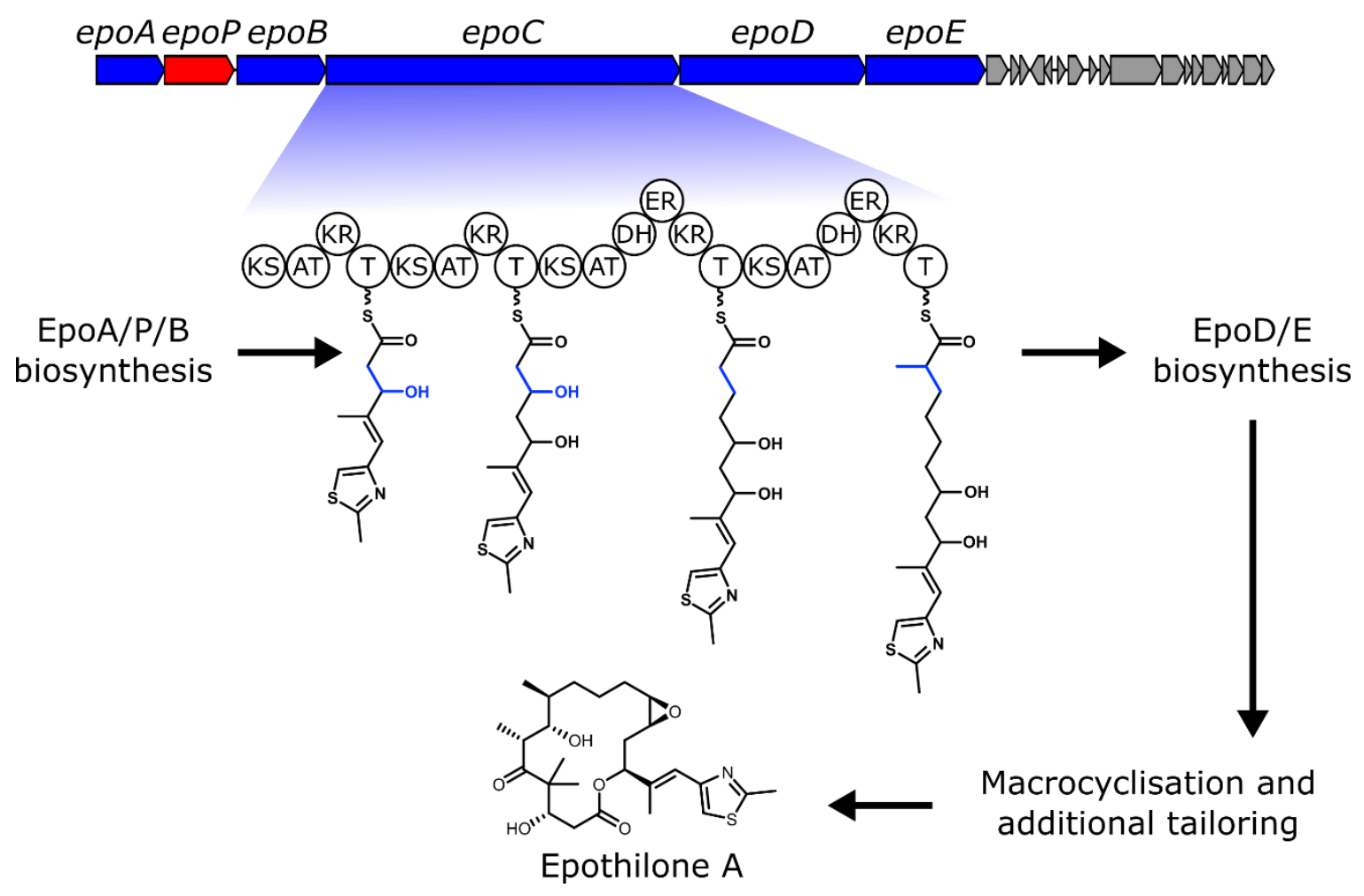

Figure 1.3 - Type I polyketide biosynthesis exemplified by epothilone. The biosynthetic gene cluster of epothilone is depicted above, with the type I polyketide genes coloured blue (epoA-E), and the single NRPS gene coloured red (epoP). The four modules of the type I PKS enzyme EpoC are then depicted, with their contribution to the growing polyketide chain coloured blue at each step. The first two modules contain a KR-domain to reduce the incorporated malonyl residue to a hydroxyl, while the final two additionally contain DH- and ER-domains to further reduce to an alkane. The final module AT-domain is selective for methylmalonyl-CoA, resulting in a branched primary carbon backbone. Formation of the thiazole ring and additional tailoring of the core structure is performed by a variety of specialised domains and enzymes, examined in further detail in Figure 1.6.

\subsubsection{Type II polyketide biosynthesis}

A minimal type II PKS consists of three enzymes, which act iteratively to produce the polyketide chain without the need for multiple modules (Figure 1.4). As in type I PKS biosynthesis, an acyl carrier protein/thiolation (ACP-/T-) domain holds the growing polyketide. Two ketosynthase proteins $\left(\mathrm{KS}_{\alpha}, \mathrm{KS}_{\beta}\right)$ function to add monomers to the growing polyketide chain ${ }^{18,42}$. $\mathrm{KS}_{\alpha}$ performs the condensation reaction, while $\mathrm{KS}_{\beta}$ (also known as the "chain length factor") halts the iterative addition of ketide units once a certain carbon chain length has been reached. Following this, the chain may be further modified by other enzymes, including cyclase, aromatase, and ketoreductase enzymes to produce core aromatic polyketide scaffolds (Figure 1.4). Tailoring functions commonly follow, 
including action by oxygenase, methyltransferase and glycosyltransferase enzymes, to decorate the core aromatic scaffold and produce diverse sets of compounds ${ }^{42,43}$.

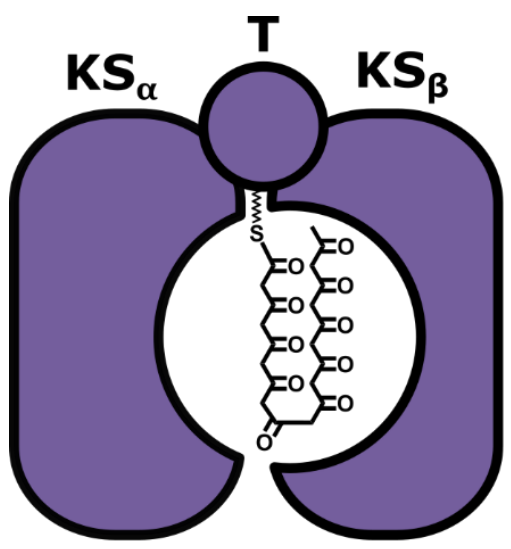
Polyketide chain release
and cyclisation<smiles>O=C1c2cc(O)cc(O)c2C(=O)C2C(O)=CC=CC12</smiles>

Anthraquinones<smiles>CC(=O)C1=C(O)Cc2cc3cc4cc(O)cc(O)c4c(O)c3c(O)c2C1=O</smiles>

Tetracyclines

Aureolic acids<smiles>CC1(O)CC(=O)c2c(cc(O)c3c2C(=O)c2c(O)cc(O)cc2C3=O)C1</smiles>

Angucyclines

Figure 1.4 - A depiction of the minimal type II PKS biosynthesis machinery, and examples of aromatic polyketide cyclisation patterns. The resulting aromatic scaffolds are often further modified by gene cluster associated tailoring enzymes to produce the final biosynthetic product. 


\subsection{Tailoring modifications}

The chemical diversity of core biosynthesised scaffolds can be further increased by tailoring functions, for both NRP and PK synthesis. These tailoring functions can occur by enzyme function in cis to core biosynthesis (occurring alongside the NRP/PK megasynth(et)ase enzymes) or trans (post release of the primary biosynthetic product) $17,32,37$

Methylation is a common modification in both NRP and PK biosynthesis. Cis-methylation (MT-) domains may be present in NRPS and type I PKS proteins, modifying the biosynthesis substrates. The substrate sites for methylation may be predicted by the location of the domain within a biosynthetic module, and the phylogeny of the domain, being classified as an N-methyltransferase (nMT-), C-methyltransferase (cMT-) or Omethyltransferase (oMT-) domain with respect to the target ${ }^{32,37}$. Methylation can also be catalysed by trans acting methyltransferase enzymes.

Amino acid side chains in NRP biosynthesis can be modified to $\beta$-hydroxy amino acids by a number of enzymes, including cytochrome P450 monooxygenases and non-heme monooxygenases of the TauD family ${ }^{32,44}$. The modified amino acids can then themselves become substrates for further modifications. For example, in ramoplanin biosynthesis the added hydroxyl group of the modified $\beta$-hydroxy aspartic acid is used as a substrate to form the lactone bond in macrocyclisation ${ }^{45}$.

Glycosylation is a common post synthesis tailoring function in both NRP and PK production ${ }^{17,32,37,46}$. In these pathways, the pre-glycosylated intermediate is termed the aglycone, which is processed by glycosyltransferases and potentially other tailoring enzymes to produce the final metabolite structure. Often the sugar substrates for glycosyltransferase enzymes are not present in the primary metabolite pool of the producer cell, and genetic pathways for the production of these non-canonical sugars may be associated with the biosynthetic gene cluster ${ }^{47}$. This is case in the chromomycin $\mathrm{A}_{3}$ biosynthetic gene cluster, where cluster associated genes encode the biosynthesis of three different deoxy sugars, along with four glycosyltransferase genes ${ }^{48}$. 


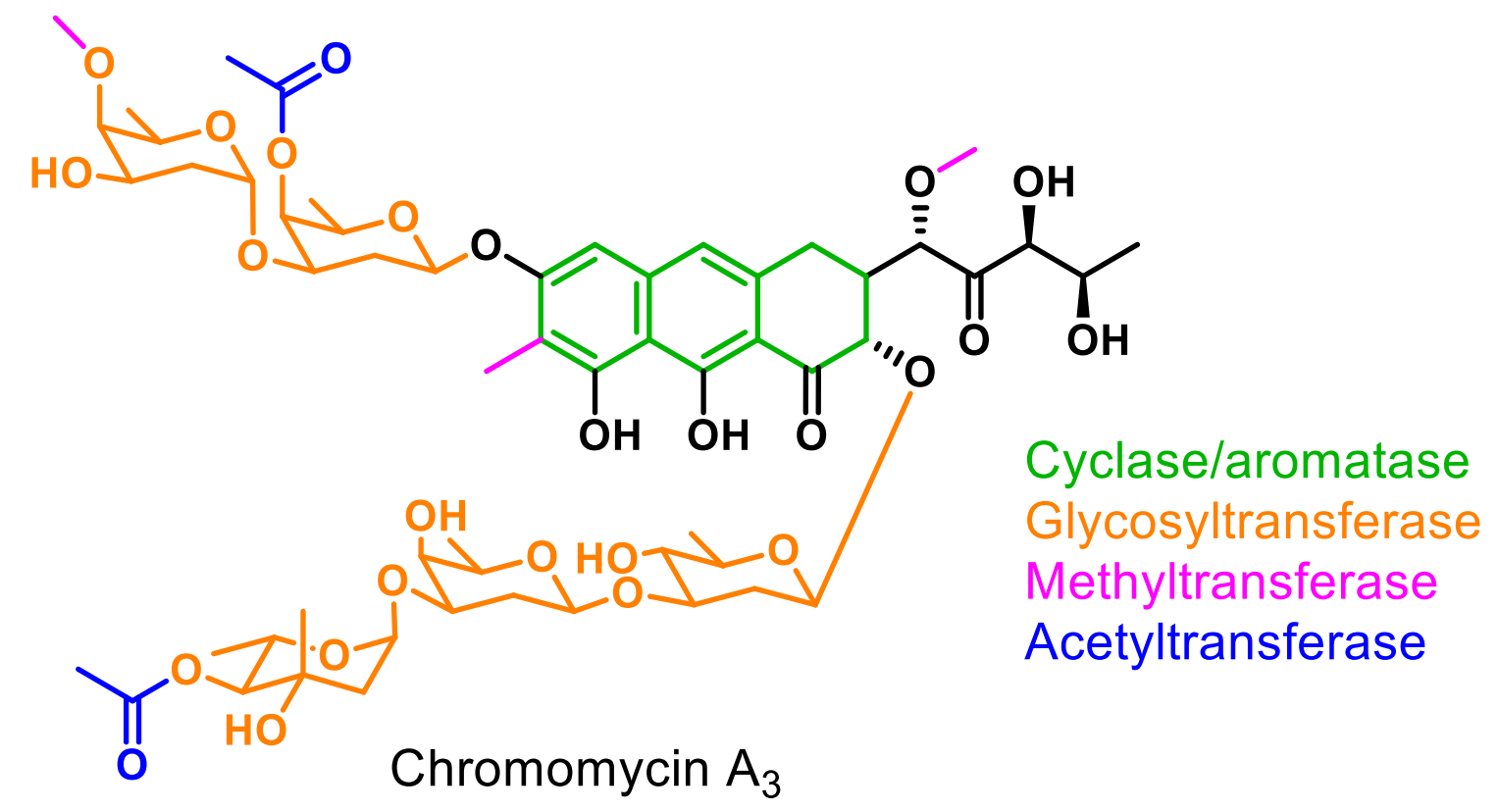

Figure 1.5 - Tailoring enzyme modifications to the aureolic acid chromomycin A3. The initial polyketide core is produced by a type II PKS, followed by cyclisation, oxidation and reduction events, along with decorations by glycosylmethyl- and acetyltransferase tailoring enzymes to produce the final bioactive compound.

Acylation of natural product intermediates is another post synthesis tailoring function represented across many biosynthesis classes ${ }^{17,49}$. N-acetyltransferases and $\mathrm{O}$ acetyltransferases have been described, acetylating natural product intermediates or monomers 50,51

Possibly the modifications that provide the greatest variation to metabolite chemical structure are the various cyclisation events. Cy-domains in NRP biosynthesis can replace C-domains in the standard NRPS module to cyclise amino acid monomers during the core peptide biosynthesis ${ }^{32,37}$. Examples of this include the formation of thiazole and oxazole residues from cysteine and serine amino acids, respectively, in the biosynthesis of epothilone, ajudazole and cystothiazole ${ }^{41,52,53}$. In each case, an Ox-domain acts subsequently to heterocyclisation to oxidise the oxazoline or thiazoline to the stable aromatic oxazole or thiazole (Figure 1.3, Figure 1.6). 

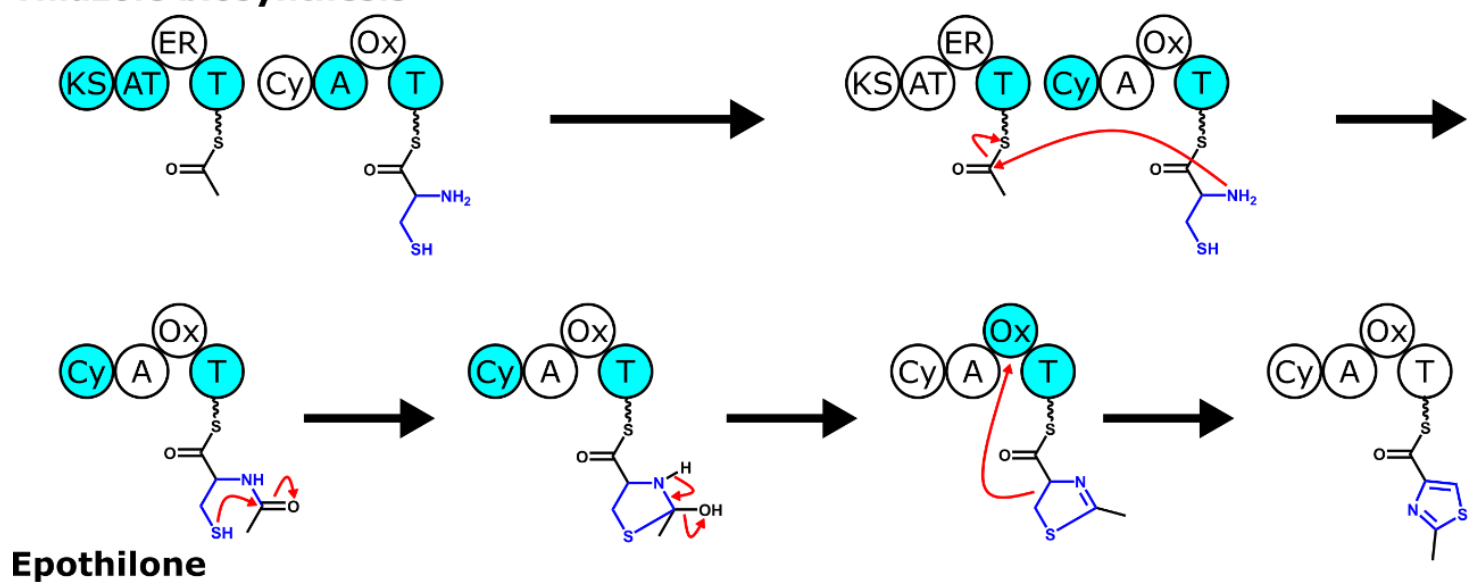

pothilone
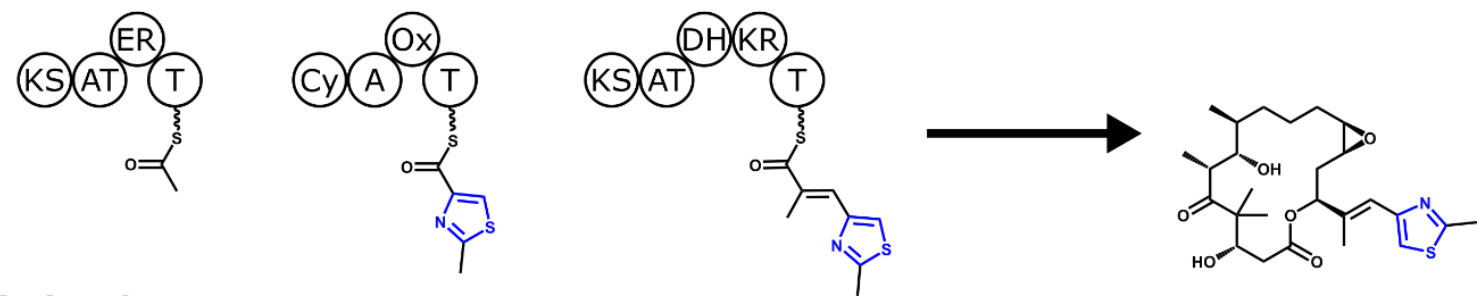

Figure 1.6 - Thiazole biosynthesis mechanism. The mechanism for the biosynthesis of the methyl-thiazole unit in epothilone is depicted, where initial monomers are loaded onto T-domains for each module (the first carrying acetyl-S residue after KS decarboxylation of malonyl group, and the second carrying cysteine as selected by the A-domain). The $\mathrm{Cy}$-domain first catalyses the formation of the $\mathrm{C}-\mathrm{N}$ bond between the two monomers, and subsequently also catalyses the cysteine cyclisation reaction. Following cyclisation, the Ox-domain desaturates to form the complete heteroaromatic moiety. Active domains at each step in biosynthesis are indicated in light blue. The preceding and post thiazole incorporation modules of epothilone biosynthesis are shown below.

Cyclisation events are key in the biosynthetic pathways of the aromatic polyketides, as previously discussed in section 1.3.2, and depicted in Figure 1.4 and Figure 1.5.

Macrocyclisation reactions are key to biosynthesis of both cyclic peptides (NRPs) and macrolides (PKs). These cyclisation events are commonly coupled to chain release from the megasynth(et)ase enzyme by a TE-domain, in both NRP and PK biosynthesis ${ }^{54}$. Intramolecular cyclisation requires an attacking nucleophile from within the metabolite chain. In NRP biosynthesis, either the N-terminal amine or a side chain amine may provide this function to produce a cyclic peptide/lactam linkage (see Figure 1.7 - polymyxin B). Alternatively, a sidechain hydroxyl may function as the TE-domain mediated nucleophile resulting in a macrolactone final product (see Figure 1.7 - daptomycin). This mechanism for TE-domain catalysed macrolactone cyclisation is the same used in PK macrolide biosynthesis (e.g. epothilone Figure 1.6). 


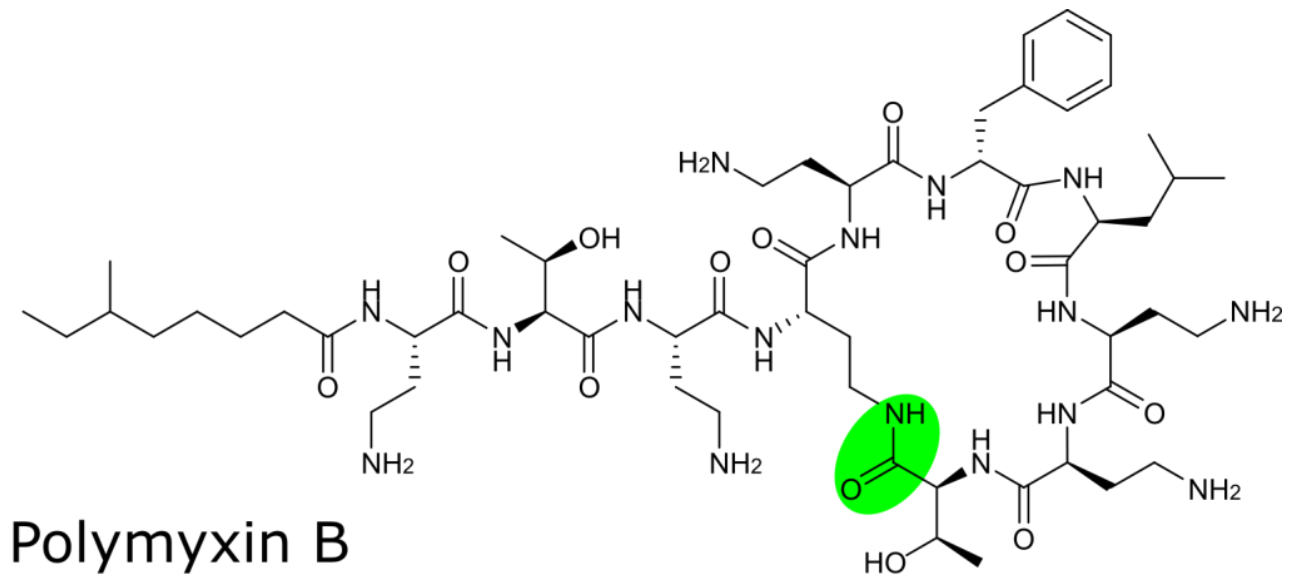

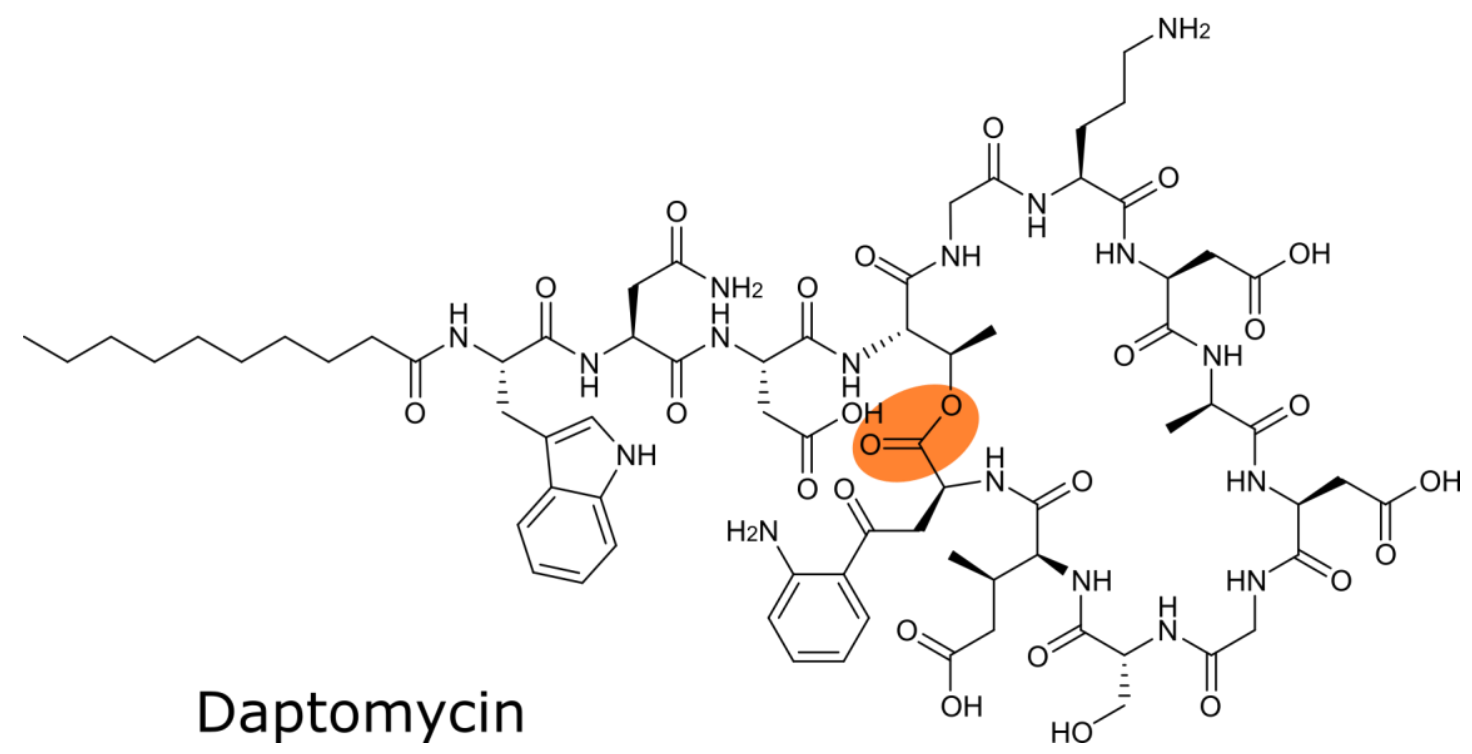

Figure 1.7 - TE-domain mediated macrocyclisation resulting in peptidolactone and peptidolactams. In polymyxin $\mathrm{B}$ biosynthesis, the deprotonated amine of the non-proteogenic diaminobutyric acid residue acts as the nucleophile to produce a cyclic peptide around the resulting lactam group (green). In daptomycin biosynthesis, the $\beta-\mathrm{OH}$ of a threonine residue acts as the nucleophile attacking group to produce a cyclic peptide around the lactone group (orange).

\subsection{Bioinformatic analysis tools}

In order to facilitate the discovery of new biosynthetic gene clusters from bacterial DNA sequence data, a number of bioinformatic tools have been produced by various research groups. These software packages can assist in not only the identification of biosynthetic gene clusters in (meta)genomic DNA sequence data, but also the annotation of genes, and prediction of the biosynthetic pathway functions. 


\subsubsection{Module monomer specificity}

For bioinformatic examination of NRPS pathways, the key analysis is prediction of the monomers selected by each A-domain, as the peptide produced from the core NRPS machinery will be a concatenation of these monomers. From the nucleotide sequence of each A-domain, the set of amino acid residues surrounding the active site binding pocket can be inferred, and used to predict monomer selectivity ${ }^{55,56}$. This was first recognised as the "Stachelhaus code", where the ten residues forming the amino acid binding pocket of an A-domain were used to predict substrate specificity ${ }^{55}$. This principle has since been expanded upon to yield many different methods for prediction of A-domain substrates. For example, Hidden Markov Model (HMM) profiles have been developed from A-domain sequences of known substrate specificity ${ }^{57,58}$. Others have used support-vector machines (SVMs), trained on sets of experimentally characterised A-domain sequences to produce tools that can predict A-domain specificity from sequence data input ${ }^{59}$, including the widely used NRPSPredictor ${ }^{60}$. These different approaches have different strengths and weaknesses, with accuracy varying according to the relationship between the query sequences and those in the datasets used in the algorithm generation. This fuelled the creation of the "ensemble" method program SANDPUMA, which uses a decision tree to apply each of the Stachelhaus active site motif system ${ }^{55}$, HMMs ${ }^{57,58}$ and SVMs ${ }^{60}$ to predict A-domain specificity with higher degrees of accuracy ${ }^{61}$.

The selectivity of AT-domains in type I PKS biosynthesis is analogous to that of the Adomains in NRPS systems. The active site amino acids of the AT-domain can likewise be used to predict the monomer selectivity, with 13 conserved active site residues, and a further 11 more distant residues implicated in substrate selection ${ }^{40}$. These amino acid positions are considered by the program SEARCHPKS, to predict AT-domain selectivity and domain organisation ${ }^{62}$. HMMs have also been developed to assess AT-domain selectivity ${ }^{57}$. Beyond substrate selection, type I PKS prediction relies on domain arrangement and the successive reduction reactions these domains may catalyse ${ }^{18,40}$.

\subsubsection{Integrated packages}

Individual monomer prediction systems have been integrated into wrapper packages for genome mining that can return full biosynthetic gene cluster identification from sequence data, putatively assign gene function, and provide metabolite predictions. Linking genetic 
information to chemical metabolite output in an accessible and accurate manner is key to sequence-first approaches to natural product discovery, providing crucial information for dereplication to avoid consistent rediscovery of the same molecules. The antibiotics and secondary metabolites analysis shell (antiSMASH) ${ }^{22,63-65}$, and prediction informatics for secondary metabolomes (PRISM) ${ }^{23,66}$, are the major pipelines used for biosynthetic gene cluster identification and prediction. The basic workflows for each involve a user-provided (meta)genome sequence being analysed for gene prediction, and then identification of gene clusters based on HMMs of signature genes (e.g., NRPS biosynthetic gene cluster constraints include the presence of $\mathrm{C}$ - and A-domains within a set distance). Genes within cluster boundaries are then fed into prediction algorithms, compared to database gene cluster sequences, and the full output from all analyses is provided in a user-friendly form.

These workflows also involve examination of the provided gene cluster sequences against reference sets, to aid in determination of function and novelty. The minimum information about a biosynthetic gene cluster (MIBiG) data standard and associated repository define a framework of biosynthetic gene cluster annotations ${ }^{21}$. Searching candidate gene clusters against centralised repositories like $\mathrm{MIBiG}$ can inform researchers about previously characterised gene functions and "sub-clusters" relevant to their candidate clusters. AntiSMASH contains on-board analysis parameters to perform these searches, via clusterBLAST and subclusterBLAST ${ }^{64}$.

The value of these platforms is especially evident for metagenome analysis. During dereplication triage stages, prioritisation of gene clusters that contain novel functional modules, A-domain sequences and gene arrangements can occur to guide selection of gene clusters that are most likely to encode synthesis of novel metabolites for expression trials.

\subsection{Genome mining}

Bioinformatic analysis of DNA sequence data from cultivated microbes has led to the discovery of many "metabolically silent" biosynthetic gene clusters in highly studied actinomycete species ${ }^{11,26-29}$. Of particular note is the $S$. coelicolor genome - prior to full genome sequence analysis, production of only 5 natural products had been achieved after 50 years of study. An additional 20 biosynthetic gene clusters were identified beyond those responsible for the known compounds after genome mining ${ }^{11}$. Through activation of these 
silent and cryptic gene clusters, compounds including the NRP iron chelator coelichelin and the polyketide coelimycin P1 have since been discovered ${ }^{67,68}$.

The genetic factors governing biosynthetic gene cluster expression and secondary metabolite production are complex, however molecular activation and heterologous expression have in some cases proven to be effective strategies to circumvent this complexity ${ }^{12,13}$.

\subsubsection{Environmental metagenome mining}

One criticism of traditional bacterial natural product discovery strategies is an overreliance on specific sub-sets of readily cultivable bacteria from soil samples - in particular the streptomycetes. These were the bacteria targeted by the Waksman platform, and as such Streptomyces species account for over two thirds of the known antibiotics ${ }^{69}$. Recent surveys into bioactive metabolite production by non-Streptomyces actinomycetes (e.g. Nocardia spp.) and non-actinomycete taxa (e.g. myxobacteria) have revealed not just an abundance of biosynthetic capacity, but also a substantially non-overlapping set of metabolite diversity ${ }^{70,71}$. By applying evolutionary and ecological principles to drug discovery efforts, many other sources for bacterial natural product discovery become attractive ${ }^{72}$. Indeed, these views are supported by a recent study into the presence of biosynthetic gene clusters in untargeted soil DNA sequence data, where many biosynthetic clusters in their assembled contigs grouped in non-actinobacterial clades ${ }^{16}$. This highlights another advantage of metagenomics strategies - that they can provide an unbiased view of the total microbial complement of an environment.

The importance of this was demonstrated in a recent study, where Jillian Banfield's team characterised thousands of biosynthetic gene clusters from hundreds of near-completely assembled bacterial genomes from soil metagenomes ${ }^{16}$. While the total species density in typical soils is commonly cited as two orders of magnitude greater than this ${ }^{8,9}$, a thorough investigation into the biosynthetic potential of microbes represented at this shotgun sequencing depth provided compelling evidence as to the abundance of biosynthetic gene clusters that can be discovered from uncultivated bacteria. Using an antiSMASH based analysis pipeline ${ }^{65}$, they discovered 1,159 biosynthetic gene clusters on assembled contigs $>10 \mathrm{~kb}, 240$ of which were NRPS, PKS or hybrid clusters ${ }^{16}$. Furthermore, the gene clusters identified in the sequencing data were of low cluster homology to those in known 
biosynthetic gene cluster databases, with 220 out of 240 sequenced NRPS and PKS clusters having less than $50 \%$ of their genes in common with any gene cluster in the MIBiG database ${ }^{21}$ (at a 50\% gene similarity cut-off level). To validate these results, the authors sought to investigate whether the gene clusters described were expressing the encoded natural product biosynthesis enzymes. A metatranscriptomics approach was employed, wherein 120 "microcosm" experiments were set up to allow RNA transcripts to be collected and sequenced directly from soil samples in response to varying additions of glucose, methanol or water over a 24-hour period. From the 180 NRPS and PKS clusters monitored, gene expression was detected in 133 gene clusters. In one particular case of a well-characterised genome, the authors observed the expression of an NRPS gene cluster, and co-expression of response regulatory genes and genes involved in cell surface remodelling, stress response, and antibiotic resistance ${ }^{16}$. This study not only validates the potential of available tools to accurately predict gene clusters from highly heterogeneous sequence data, but also provides insight into the biological and ecological roles bacterial natural products can play in their native host's response to environmental changes.

\subsection{Heterologous expression}

While the above approach demonstrates the ability to predict biosynthetic gene cluster sequence and observe expression, accessing bioactive compounds in useful quantities often requires dedicated fermentation. A core premise underpinning metagenomics is that as very few environmental bacteria can be effectively cultured in the lab environment, fermentation studies must be carried out in heterologous expression hosts. Recently a number of optimised expression hosts for large biosynthetic gene clusters have become available, with expression of potentially contaminating native biosynthetic clusters having been knocked out, together with other modifications that permit higher levels of production of desired compounds. These hosts include the Streptomyces species coelicolor ${ }^{73,74}$, avermitilis ${ }^{75}$, lividans ${ }^{76}$, and albus ${ }^{77}$. This offers a means to link gene cluster discovery with heterologous expression, via creation of large insert metagenome DNA libraries that are screened for biosynthesis genes, and then cloned into biosynthetically gifted expression hosts. 


\subsection{Metagenome library screening}

The creation of genetic libraries from metagenome samples provides many advantages over direct genetic screening of environmental DNA. Genetic libraries, when appropriately stored in E. coli hosts, are stable, renewable genetic resources for preserving the encoded information. Libraries can be arrayed into sub-pools to deconvolute the genetic information, which is particularly important for complex soil bacteria samples where species can number on the order of $10,000{ }^{8,9}$. Deconvoluted screening pools can make sequence based screening efforts more tractable ${ }^{78,79}$. Storing the library in such vectors also allows for the possibility of functional screening experiments, where expression of genes from the stored library can be used to infer information about the stored DNA content. And finally, when libraries are formed in large insert vector units, it can be a relatively simple process to convert the stored DNA into an expression unit, to permit heterologous expression of the encoded biosynthetic gene cluster.

\subsubsection{DNA sequence homology screening}

Sean Brady's group have extensively deployed homology based screening approaches, in particular by PCR screening metagenome cosmid libraries using degenerate primers that target conserved motifs sequences within A- and KS-domains. They have shown that sequencing of these PCR amplicons (natural product sequence tags) then provides insight into the diversity and phylogeny of NRPS gene clusters, allowing information about the relatedness of the metabolite structure to be inferred ${ }^{78-81}$. This permits a layer of prioritisation and dereplication before recovering gene clusters from the metagenome libraries for heterologous expression.

In an early demonstration of this approach, a single arrayed soil metagenome cosmid library was screened with degenerate PCR primers to recover 16,949 unique A-domain and 4,167 KS-domain sequences ${ }^{78}$. Based on these conserved domain sequences, the biosynthetic gene clusters that contained them were classified into predicted biosynthetic classes. Subsequent full sequencing of the recovered cosmids then validated these biosynthetic class predictions made from the conserved domain sequences with $81 \%$ accuracy ${ }^{78}$. In two recent applications of the sequence tag screening approach for NRPS discovery, the Brady group sought to target conserved A-domain sequences for calcium dependent antibiotics in their cosmid metagenome library sequences ${ }^{80,81}$. These studies culminated in the 
discoveries of two new antibiotics- the malacidins and cadasides - each of which possessed antibacterial activity against methicillin-resistant Staphylococcus aureus (MRSA).

PCR based screening has also revealed that soil metagenomes contain a rich diversity of type II PKS systems ${ }^{82,83}$. Using conserved primer sets for $\mathrm{KS}_{\alpha}$ or $\mathrm{KS}_{\beta}$ genes ${ }^{84,85}$, aromatic polyketide gene clusters have been recovered from soil metagenomes and found to produce compounds with diverse and novel structures ${ }^{82,86,87}$. These include the novel pentacyclic polyketide erdacin, which is a potent antioxidant ${ }^{86}$. Another metagenome derived type II PKS pathway produced fasamycins A and B by heterologous expression in S. albus ${ }^{87}$. These compounds were found to have antibacterial activity against the widely resistant pathogens MRSA and vancomycin-resistant Enterococcus faecalis (VRE).

The data generated from these screening campaigns has also been interrogated for higher level analysis on the abundance and diversity of natural product metabolism in soil microbiomes. Using short A- and KS-domain tag sequencing, different soil environments have been evaluated for biosynthetic diversity, finding that the distance between sample sites, changes in latitude, and changes in biome are the major contributors to observed diversity differences between samples sites ${ }^{14,15,88}$.

A different approach was recently employed by Belinda Ferrari and her team, who used long read Pacbio sequencing to interrogate sequence tags from polar desert soils ${ }^{89}$. The authors reasoned that the use of long read data to cover the entire PCR amplicon sequence ( 700 bp A-domain, and 1200-1400 bp KS/AT domains) could provide more information into gene cluster function and phylogeny than the short read sequencing previously used by the Brady group ( 200-400 bp) ${ }^{14}$. Using their long-read amplicon screening, the Ferrari team found many biosynthesis gene sequences with low sequence homology to known gene clusters, and an abundance of syringomycin/gramicidin like gene cluster sequences, suggesting richness of lipopeptide antibiotic diversity in polar soils. Other sequences indicated the presence of compounds similar to the antibiotic actinomycin, and anti-tumour agents bleomycin and epothilone. Of note, human impacted sites displayed the lowest biosynthetic diversity in sequence data ${ }^{89}$. This may indicate that the use of more isolated sample sites and deeper analytical techniques will promote recovery of novel biosynthetic diversity. 


\subsubsection{Functional screening}

Functional screening of metagenome libraries seeks to identify clones of interest by the expression of gene(s) from the metagenome insert. This can relate to the expression of an entire gene cluster and observation of metabolite output, or a screen may employ complementation/reporter strategies to observe expression of a single key gene of interest.

In the search for natural product biosynthesis pathways, the most obvious functional screening technique is to observe production of secondary metabolites from full pathway expression. This normally involves cloning a library into a screening/expression host, and observing metabolites directly (e.g. changes to colony morphology or colour), or their effects (toxicity of extracts or overlaid test strains).

Heterologous expression from metagenome libraries often relies on cooperation between the host and metagenome native transcriptional machinery, therefore host selection is an important factor. Many such experiments have relied on expression in various E. coli strains, which has proven to be adept at expression of biosynthetic gene clusters from diverse sources ${ }^{90,91}$. Sean Brady has extensively employed a strategy of constructing metagenome libraries within $E$. coli, followed by plating on agar to develop colonies, which may produce secondary metabolites derived from the metagenome insert. These can be detected visually, or by observing clearance zones when overlaid with a "test strain", such as Bacillus subtilis ${ }^{92}$. The Brady group has also extended this direct expression strategy to a diverse set of Proteobacteria expression host strains ${ }^{93,94}$, and to the actinomycete $S$. albus ${ }^{95}$. Others have attempted to extend the capacity for $E$. coli expression of natural product gene clusters in these screening platforms by co-expression of alternative RNA polymerase sigma factors, to allow E. coli to express genes from a more diverse set of promoter sites 96,97. Similar regulatory control modulation led to the discovery of the MRSA-active antibiotic tetarimycin, by overexpressing a Streptomyces antibiotic regulatory protein to upregulate biosynthesis gene expression ${ }^{98}$.

In addition to the overlay experiments, others have used complementation or reporter gene systems, to screen for metagenome clones containing genes linked to secondary metabolism. The most notable of these strategies is based around PPTase genes. As previously described (sections 1.2 and 1.3.1), PPTase enzymes catalyse a post translational modification to T-domains of both NRPS and PKS enzymes, which is required for enzyme activation and subsequent biosynthesis. As such these genes are often associated with 
biosynthetic gene clusters, meaning the recovery of a PPTase gene containing metagenome clones often results in the recovery of other biosynthesis genes. This strategy was first developed by Owen and Robins et al., where their PPTase screen in an E. coli host was reported by the activation of the NRPS blue pigment synthetase A from Streptomyces lavendulae, producing the blue pigment indigoidine ${ }^{99}$. These blue colonies could be picked to facilitate the recovery of PPTase gene containing metagenome clones. The Brady group later transferred this indigoidine screening technique to a $S$. albus host, facilitating this screening in a secondary metabolite producer strain ${ }^{100}$.

In addition to visual detection methods, PPTase expression can be used as a selection, when PPTase expression is required for cell growth and survival. This was the basis for the PPTase screen first described in Charlop-Powers at al. 2013, where an E. coli strain with its native PPTase gene knocked out $(\Delta e n t D)$ requires complementation of another PPTase to activate the NRPS pathway for a siderophore (enterobactin) under iron-restricted conditions ${ }^{101}$. In absence of a metagenome derived PPTase gene, enterobactin cannot be produced, and the cells do not acquire sufficient iron to support growth and division. Colony production is therefore selective for cells containing a metagenome derived PPTase gene. This high-throughput screening platform was used to enrich a metagenome library for clones containing PPTases, and the linked secondary metabolic gene clusters ${ }^{101}$.

\subsection{Aims of this study}

The overall aim of this study was to investigate the secondary metabolite production potential of soil bacteria in New Zealand. The specific project aims were:

1. To generate large metagenome libraries from environmental samples sourced across New Zealand.

2. To interrogate those libraries to find and recover novel biosynthetic gene clusters.

3. To investigate these gene clusters for novel biosynthesis signatures relative to known gene cluster sequences.

4. To select metagenome derived biosynthetic gene clusters for heterologous expression studies and characterise the major pathway specific metabolites. 


\subsection{Materials and Methods}

\subsection{General microbial strains and materials}

All reagents were of analytical or molecular biology grade, obtained from Sigma Aldrich unless otherwise noted. Dilutions were performed using distilled de-ionised water $\left(\mathrm{ddH}_{2} \mathrm{O}\right)$ unless otherwise stated. Solution $\mathrm{pH}$ was adjusted using $10 \mathrm{M} \mathrm{HCl}$ or $10 \mathrm{M} \mathrm{NaOH}$. Sterilisation was performed by autoclave, or by filtration through $0.22 \mu \mathrm{m}$ syringe filter. Media supplements and antibiotics were added to media following autoclaving.

\subsubsection{Bacterial and yeast strains}

The Escherichia coli, Streptomyces albus, Bacillus subtilis, and Saccharomyces cerevisiae strains used in this study are described in Table 2.1.

\begin{tabular}{|c|c|c|}
\hline Strain & Description & Source \\
\hline $\begin{array}{l}\text { E. coli DH10 } \\
\text { (EC100) }\end{array}$ & $\begin{array}{l}\mathrm{F}-\text { mcrA } \Delta(m r r-h s d \mathrm{RMS}-m c r \mathrm{BC}) \\
\phi 80 \mathrm{~d} l a c \mathrm{Z} \Delta \mathrm{M} 15 \Delta \text { lacX74 recA1 end } \mathrm{A} 1 \text { ara } \mathrm{D} 139 \\
\Delta(\text { ara, leu }) 7697 \text { gal } \mathrm{U} \text { galK } \lambda-\text { rpsL nup } \mathrm{G}\end{array}$ & Epicentre \\
\hline E. coli $\mathrm{DH} 10 \beta \Delta e n t D$ & DH10 $\Delta e n t D$ & 101 \\
\hline E. coli $\mathrm{EPI} 300$ & $\begin{array}{l}\mathrm{F}-\text { mcrA } \Delta(m r r-h s d \mathrm{RMS}-m c r \mathrm{BC}) \\
\phi 80 \mathrm{~d} l a c \mathrm{Z} \Delta \mathrm{M} 15 \Delta \text { lacX74 recA1 end } 1 \text { 1 araD139 } \\
\Delta(\text { ara, leu }) 7697 \text { galU galK } \lambda-\text { rpsL nupG trfA } \\
\text { dhfr }\end{array}$ & Epicentre \\
\hline E. coli $\mathrm{S} 17-1$ & recA pro hsdR RP42 (Tc::Mu; Km::Tn7) & 102 \\
\hline E. coli NM 759 & $\begin{array}{l}\text { recA56 } \Delta(\mathrm{mcrA}) e 14^{\circ} \Delta(\mathrm{mrr}-\mathrm{hsd}-\mathrm{mcr})(\lambda \mathrm{imm} 434 \\
\text { clts b2 } \mathrm{red} 3 \mathrm{Dam} 15 \mathrm{Sam} 7) / \lambda\end{array}$ & 103 \\
\hline E. coli $B H B 2688$ & N205 recA- $(\lambda i m m 434$ cIts b2 red Eam4 Sam7)/ $\lambda$ & 104 \\
\hline E. coli $7 N T$ & $\begin{array}{l}\mathrm{W} 3110 \Delta n f_{s} A \Delta n f_{s} B \text { yieF } \Delta y c a K \Delta m d a B \Delta a z o R \\
\Delta n e m A \Delta \text { tolC }\end{array}$ & 105 \\
\hline S. albus $\mathrm{J} 1074$ & $\begin{array}{l}\text { Isoleucine and valine auxotrophic derivative of } S \text {. } \\
\text { albus G lacking SalI-restriction activity }\end{array}$ & 106 \\
\hline
\end{tabular}


B. subtilis 168 $\triangle \operatorname{trp} C 2$

S. cerevisiae BY4727

MAT $\alpha$

his $3 \Delta 200::$ leu $2 \Delta 0::$ lys $2 \Delta 0:: \operatorname{met} 15 \Delta 0:: \operatorname{trp} 1 \Delta 63::$ ur a3 $\Delta 0 \Delta \operatorname{dn} 14$

S. cerevisiae $\triangle P D R \quad$ Yeast deletion mutant with efflux knockout y7092 MAT $\alpha$ PDR1 $\triangle$ PDR3

Table 2.1 - Bacterial and yeast strains used in this study

\subsubsection{Plasmids vectors}

\begin{tabular}{|c|c|c|}
\hline Vector & Relevant characteristics & Source \\
\hline pWEB & Cosmid vector. ColE1ori, $\mathrm{Kan}^{\mathrm{R}}, \mathrm{Amp}^{\mathrm{R}}$, cos & Epicentre \\
\hline pWEB_CH & pWEB + CH eDNA cosmid library & This study \\
\hline pWEB::tnc & Cosmid vector. ColE1ori, $\mathrm{Chl}^{\mathrm{R}}, \mathrm{Amp}^{\mathrm{R}}$, cos & Epicentre \\
\hline pWEB::tnc_HM & pWEB::tnc + HM eDNA cosmid library & This study \\
\hline pWEB::tnc_RX & pWEB::tnc + RX eDNA cosmid library & This study \\
\hline pWEB::tnc_SL & pWEB::tnc + SL eDNA cosmid library & This study \\
\hline pWEB::tnc_D9 & pWEB::tnc cosmid recovered from HM library & This study \\
\hline pWEB::tnc_D9_RF & $\begin{array}{l}\text { pWEB::tnc_D9 retrofitted with DraI digest } \\
\text { fragment from pOJ436 }\end{array}$ & This study \\
\hline pWEB::tnc_D3 & pWEB::tnc cosmid recovered from HM library & This study \\
\hline pWEB::tnc_D3_T7OL & pWEB::tnc cosmid recovered from HM library & This study \\
\hline pWEB::tnc_D3_M13OL & pWEB::tnc cosmid recovered from HM library & This study \\
\hline pOJ436 & $\begin{array}{l}\phi \mathrm{C} 31 \text { attB-int-derived integration vector. Used } \\
\text { as source of OriT, } \mathrm{Apr}^{\mathrm{R}} \text {, Integrase for } \\
\text { retrofitting pWEB::tnc for Streptomyces } \\
\text { integration }\end{array}$ & 110 \\
\hline pTARa & $\begin{array}{l}\text { BAC based } E \text {. coli/S. cerevisiae/Streptomyces } \\
\text { shuttle capture vector. } \phi \mathrm{C} 31 \text { attB-int-derived } \\
\text { integration vector. E. coli maintenance and } \\
\text { selection; oriV, } \mathrm{Chl}^{\mathrm{R}} \text {, Streptomyces integration }\end{array}$ & 111 \\
\hline
\end{tabular}




\begin{tabular}{lll} 
& and selection; OriT, $\mathrm{Apr}^{\mathrm{R}}$, Integrase, $S$. \\
& cerevisiae maintenance and selection; ARSH4, \\
& CEN6, URA3. & \\
pTARa_D3_2 & This study \\
& pWEB::tnc_D3 and pWEB::tnc_D3_M13OL & \\
pIJ10257 & $\phi$ BT1 attP-int-derived integration vector with & 112 \\
& Streptomyces constitutive expression cassette & \\
& (ermE promoter, RBS, MCS) and Hyg ${ }^{\mathrm{R}}$. & \\
pIJ10257_OIVW & pIJ10257 + D3 OIV and W genes & This study \\
\hline
\end{tabular}

Table 2.2 - Plasmids used in this study

\subsubsection{Oligonucleotide primers}

Macrogen Inc. synthesised all primers used in this study. Primers were reconstituted to a final concentration of $100 \mu \mathrm{M}$ in a $1 \mathrm{x}$ TE buffer for storage at $-20{ }^{\circ} \mathrm{C}$, with working stocks prepared by dilution to $10 \mu \mathrm{M}$ in sterile $\mathrm{ddH}_{2} \mathrm{O}$. In general, primers were designed manually in Geneious software. Primers for cosmid overlap recovery were designed using Primer3Plus. Primers for Gibson assembly were designed using the NEBuilder Assembly Tool and altered as appropriate in Geneious. Primers not designed in the course of this study are indicated in the legend of Table 2.3.

\begin{tabular}{ll}
\hline Primer name & Sequence $\left(\mathbf{5}{ }^{\prime} \rightarrow \mathbf{3}\right.$ ') \\
\hline T7promoter ${ }^{\text {a }}$ & TAATACGACTCACTATAGGG \\
M13F-pUC a & GTTTTCCCAGTCACGAC \\
KS $_{\text {a_f }}{ }^{\text {b }}$ & TSGCSTGCTTGGAYGCSATC \\
KS $_{\alpha \_r}{ }^{\text {b }}$ & TGGAANCCGCCGAABCCGCT \\
D9_T7_f & ACGTTCTTCACCCAGTCGGA \\
D9_T7_r & AAGCCAGGTCTCCTGCAACA \\
D9_KS_f & TGGACTACGTACCGCTGAAG \\
D9_KS_r & TTCGTTCTGGCCGAACTCGA \\
D9_M13_f & ATGTTCACCGTGCTGTTCGC \\
D9_M13_r & TTCTGTGCCATGTGATCCGC
\end{tabular}




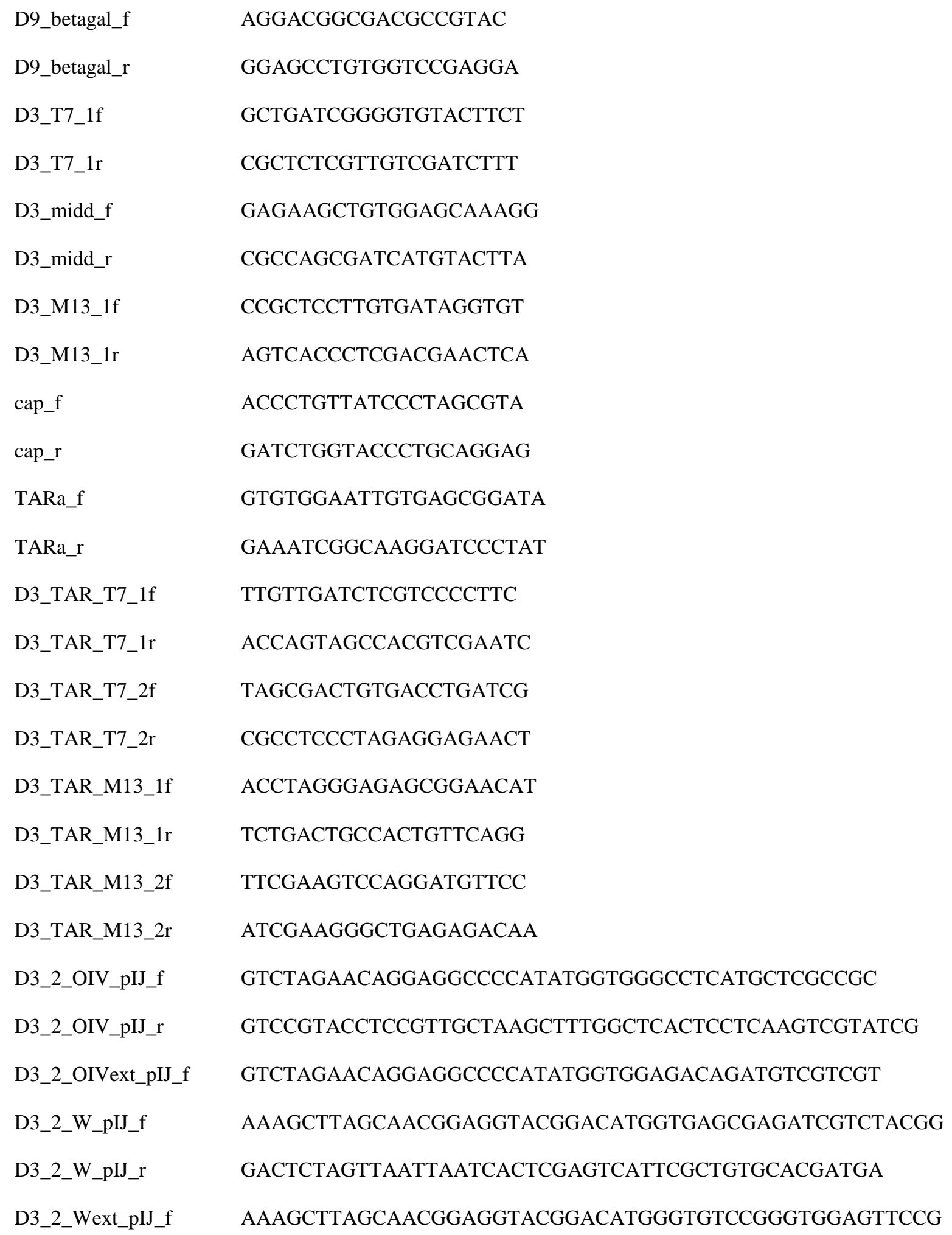




\subsubsection{Media}

Media was sterilised by autoclaving for 20 minutes at $121{ }^{\circ} \mathrm{C}$. Supplements and antibiotics were added to cool media post sterilisation. Solid media followed the below liquid media recipes with agar added prior to sterilisation to either $1.5 \%(\mathrm{w} / \mathrm{v})$ or $2 \%(\mathrm{w} / \mathrm{v})$. Media denoted with an asterisk $(*)$ were purchased as premixed powders.

Low salt lysogeny broth (LB) medium *: Tryptone $10 \mathrm{~g} / \mathrm{L}, \mathrm{NaCl} 5 \mathrm{~g} / \mathrm{L}$, yeast extract $5 \mathrm{~g} / \mathrm{L}$ Tryptic soy broth (TSB) medium *: Tryptone $17 \mathrm{~g} / \mathrm{L}$, phytone (soybean digest) $3 \mathrm{~g} / \mathrm{L}$, glucose $2.5 \mathrm{~g} / \mathrm{L}, \mathrm{NaCl} 5 \mathrm{~g} / \mathrm{L}, \mathrm{K}_{2} \mathrm{HPO}_{4} 2.5 \mathrm{~g} / \mathrm{L}$

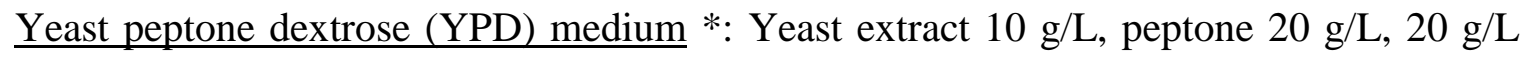
glucose

Iron limited screening agar: 1x M9 salts $\left(\mathrm{Na}_{2} \mathrm{HPO}_{4} 6.78 \mathrm{~g} / \mathrm{L}, \mathrm{KH}_{2} \mathrm{PO}_{4} 3 \mathrm{~g} / \mathrm{L}, \mathrm{NH}_{4} \mathrm{Cl} 1 \mathrm{~g} / \mathrm{L}\right.$, $\mathrm{NaCl} 0.5 \mathrm{~g} / \mathrm{L}$ ), $2 \mathrm{mM} \mathrm{MgSO} 4,100 \mu \mathrm{M} \mathrm{CaCl}_{2}$, glucose $4 \mathrm{~g} / \mathrm{L}$, casamino acids $1 \mathrm{~g} / \mathrm{L}, 100 \mu \mathrm{M}$ 2-2 dipyridyl, $10 \mu \mathrm{M}$ thiamine- $\mathrm{HCl}$

Note: all components must be sterilised separately, and combined post autoclave, immediately prior to pouring agar plates. Media should appear colourless - if all components are autoclaved in combination, media will appear brown and be unusable.

NZY medium: Casein digest $10 \mathrm{~g} / \mathrm{L}$, yeast extract $5 \mathrm{~g} / \mathrm{L}, \mathrm{NaCl} 5 \mathrm{~g} / \mathrm{L}, \mathrm{MgCl}_{2} .6 \mathrm{H}_{2} \mathrm{O} 0.1 \mathrm{~g} / \mathrm{L}$, pH 7.0

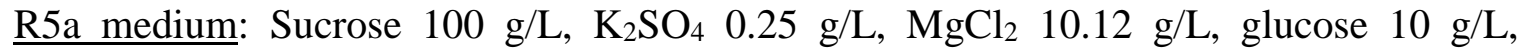
casamino acids $0.1 \mathrm{~g} / \mathrm{L}$, MOPS, $21 \mathrm{~g} / \mathrm{L}$, yeast extract $5 \mathrm{~g} / \mathrm{L}$, trace elements A $2 \mathrm{~mL} / \mathrm{L}, \mathrm{pH}$ 6.9

International Streptomyces Protocol medium 4 (ISP4): Starch 10 g/L, K2 $\mathrm{HPO}_{4} 1 \mathrm{~g} / \mathrm{L}$, $\mathrm{MgSO}_{4} .7 \mathrm{H}_{2} \mathrm{O} 1 \mathrm{~g} / \mathrm{L}, \mathrm{NaCl} 1 \mathrm{~g} / \mathrm{L}, \mathrm{NH}_{4} \mathrm{SO}_{4} 2 \mathrm{~g} / \mathrm{L}, \mathrm{CaCO}_{3} 2 \mathrm{~g} / \mathrm{L}$, trace elements B $1 \mathrm{~mL} / \mathrm{L}$, pH 7.2

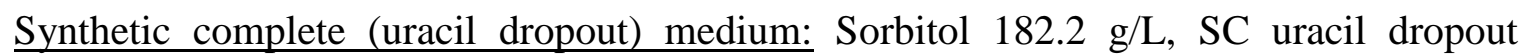
supplement $1.92 \mathrm{~g} / \mathrm{L}$, yeast nitrogen base $6.7 \mathrm{~g} / \mathrm{L}$, glucose $20 \mathrm{~g} / \mathrm{L}$ 


\subsubsection{Media supplements}

Supplements were dissolved in $\mathrm{ddH}_{2} \mathrm{O}$ unless otherwise stated. Antibiotic stock concentrations were made to 1000x the working concentration in media. Supplements were sterilised by filtration through $0.22 \mu \mathrm{m}$ syringe filter.

1000x ampicillin: $200 \mathrm{mg} / \mathrm{mL}$

1000x kanamycin: $50 \mathrm{mg} / \mathrm{mL}$

1000x chloramphenicol: $34 \mathrm{mg} / \mathrm{mL}$ in $95 \%$ ethanol

1000x apramycin: $50 \mathrm{mg} / \mathrm{mL}$

1000x hygromycin: $100 \mathrm{mg} / \mathrm{mL}$

Nalidixic acid: $25 \mathrm{mg} / \mathrm{mL}$ (10 M NaOH added dropwise to dissolve)

500x trace elements A: $\mathrm{ZnCl}_{2} 40 \mathrm{~g} / \mathrm{L}, \mathrm{FeCl}_{3} .2 \mathrm{H}_{2} \mathrm{O} 200 \mathrm{~g} / \mathrm{L}, \mathrm{CuCl}_{2} .2 \mathrm{H}_{2} \mathrm{O} 10 \mathrm{~g} / \mathrm{L}$, $\mathrm{MnCl}_{2} .2 \mathrm{H}_{2} \mathrm{O} 10 \mathrm{~g} / \mathrm{L}, \mathrm{Na}_{2} \mathrm{~B}_{4} \mathrm{O}_{7} \cdot 10 \mathrm{H}_{2} \mathrm{O} 10 \mathrm{~g} / \mathrm{L},\left(\mathrm{NH}_{4}\right) 6 \mathrm{Mo}_{7} \mathrm{O}_{24} .4 \mathrm{H}_{2} \mathrm{O} 10 \mathrm{~g} / \mathrm{L}$

1000x trace elements B: $\mathrm{CaCO}_{3} 100 \mathrm{~g} / \mathrm{L}, \mathrm{FeSO}_{4} .7 \mathrm{H}_{2} \mathrm{O} 100 \mathrm{~g} / \mathrm{L}, \mathrm{ZnSO}_{4} .7 \mathrm{H}_{2} \mathrm{O} 100 \mathrm{~g} / \mathrm{L}$, $\mathrm{MnCl}_{2} .7 \mathrm{H} 2 \mathrm{O} 100 \mathrm{~g} / \mathrm{L}$

\subsubsection{Enzymes}

Restriction endonucleases, recombinant shrimp alkaline phosphatase (rSAP), NEBuilder Hifi DNA assembly mastermix, Q5 DNA polymerase mastermix, and Cas9 were obtained from New England Biolabs.

End-It DNA End-Repair Kit and Fast-link DNA ligase were obtained from Epicentre.

Biomix red mastermix was obtained from Bioline.

\subsection{Microbial growth and maintenance}

LB was used for routine growth and maintenance of all E. coli and B. subtilis. TSB was used for $S$. albus. YPD was used for S. cerevisiae. Growth temperatures used were typically: $37{ }^{\circ} \mathrm{C}$ for E. coli, $30{ }^{\circ} \mathrm{C}$ for B. subtilis, S. albus, and S. cerevisiae. For liquid cultures aeration was provided by shaking at $200 \mathrm{rpm}$. Medium-term maintenance was 
achieved by storing agar plates containing bacteria and yeast at $4{ }^{\circ} \mathrm{C}$ for up to two weeks. For long-term maintenance of strains, an aliquot of overnight culture was mixed 1:1 with $80 \%$ glycerol and stored at $-80{ }^{\circ} \mathrm{C}$. S. albus spore stocks were generated by plating strains on ISP4 agar plates and culturing for $1-2$ weeks, followed by scraping sporing bodies into sterile $\mathrm{ddH}_{2} \mathrm{O}$, filtering out mycelium through sterile cotton wool, collecting spores by centrifuge, and resuspending in sterile $20 \%$ glycerol for storage at $-20{ }^{\circ} \mathrm{C}$.

To ensure maintenance of plasmids with antibiotic resistance markers, the appropriate antibiotic(s) were added to liquid and solid media.

\subsection{General molecular biology}

\subsubsection{Isolation of plasmid and cosmid DNA}

Plasmid and cosmid DNA was isolated from overnight $E$. coli cultures in LB, using the standard Qiagen protocol with spin columns and the following buffer recipes:

Buffer P1: 50 mM Tris-HCl pH 8.0,10 mM EDTA, $100 \mu \mathrm{g} / \mathrm{mL}$ RNaseA

Buffer P2: $200 \mathrm{mM} \mathrm{NaOH}, 1 \%$ SDS

Buffer N3: 4.2 M Gu-HCl, 0.9 M potassium acetate, $\mathrm{pH} 4.8$

Buffer PB: $5 \mathrm{M} \mathrm{Gu}-\mathrm{HCl}, 30 \%$ isopropanol

Buffer PE: 10 mM Tris-HCl pH 7.5, 80\% ethanol

Buffer EB: 10 mM Tris-HCl pH 8.5

Minipreps were eluted with $60 \mu \mathrm{L}$ EB or TE buffer, preheated to $60{ }^{\circ} \mathrm{C}$.

\subsubsection{Polymerase chain reaction}

All PCRs were performed in a Bio-Rad T100 Thermal Cycler.

Diagnostic PCRs were performed using BioMix Red 2x Master Mix (Bioline). Where the product of a PCR was to be used for cloning, Q5 High-Fidelity 2x Master Mix (NEB) was used. 
Annealing temperatures were determined for each primer set based on the melting temperature of the primers in the specific PCR mixture using the manufacturers' instructions.

Biomix Red PCR components

Biomix Red PCR protocol

\begin{tabular}{ll|ll|l}
\hline Component & $\begin{array}{l}\text { Final } \\
\text { concentration }\end{array}$ & Temperature & Time \\
& $0.1-10 \mathrm{ng}$ DNA & $95{ }^{\circ} \mathrm{C}$ & $5 \mathrm{~min}$ \\
\hline DNA template & $0.5 \mu \mathrm{M}$ & $95{ }^{\circ} \mathrm{C}$ & $20 \mathrm{~s}$ & \\
Forward primer & $0.5 \mu \mathrm{M}$ & $\mathrm{T}_{\mathrm{A}}{ }^{\circ} \mathrm{C}$ & $20 \mathrm{~s}$ & 30 cycles \\
Reverse primer & $72{ }^{\circ} \mathrm{C}$ & $20 \mathrm{~s} / \mathrm{kB}$ & \\
Biomix Red 2x MM & $1 \mathrm{x}$ & $72{ }^{\circ} \mathrm{C}$ & $5 \mathrm{~min}$ & \\
$\mathrm{ddH}_{2} \mathrm{O}$ & To final volume & & \\
\hline
\end{tabular}

Table 2.4 - Standard Biomix Red PCR components and cycle protocol.

Q5 Hifi PCR components

Q5 Hifi PCR protocol

\begin{tabular}{ll|ll|l}
\hline Component & $\begin{array}{l}\text { Final } \\
\text { concentration }\end{array}$ & Temperature & Time \\
& $0.1-10 \mathrm{ng}$ DNA & $98{ }^{\circ} \mathrm{C}$ & $30 \mathrm{~s}$ & \\
\hline DNA template & $0.5 \mu \mathrm{M}$ & $98{ }^{\circ} \mathrm{C}$ & $10 \mathrm{~s}$ & \multirow{2}{*}{30 cycles } \\
Forward primer & $0.5 \mu \mathrm{M}$ & $\mathrm{T}_{\mathrm{A}}{ }^{\circ} \mathrm{C}$ & $20 \mathrm{~s}$ & \\
Reverse primer & $1 \mathrm{x}$ & $72{ }^{\circ} \mathrm{C}$ & $20 \mathrm{~s} / \mathrm{kB}$ & \\
Q5 Hifi 2x MM & To final volume & $72{ }^{\circ} \mathrm{C}$ & $5 \mathrm{~min}$ & \\
$\mathrm{ddH}_{2} \mathrm{O}$ & & &
\end{tabular}

Table 2.5 - Standard Q5 High-Fidelity PCR components and cycle protocol.

\subsubsection{Restriction endonuclease digestion}

Restriction digests were carried out according to the manufacturer's directions. Where the digest products were to be used in downstream processes (e.g. ligations, Gibson assembly) restriction enzymes were heat inactivated and the DNA recovered by isopropanol precipitation, using a Zymospin column, or gel purified as appropriate. 


\subsubsection{Gel extraction of DNA fragments}

Extraction of DNA from agarose gel $(4 \mathrm{~kb}-10 \mathrm{~kb})$ was performed using MinElute Gel Extraction kit (Qiagen) following the manufacturer's instructions. Extraction of larger DNA fragments $(10-20 \mathrm{~kb})$ from low melting point agarose gels was performed by $\beta$ Agarase I (NEB) digestion and DNA precipitation following manufacturer's instructions.

For gel purification of eDNA prior to cosmid library construction, electroelution was used to recover eDNA from agarose gels (eDNA extraction procedure 2.4.1). The eDNA sample(s) were loaded into appropriate sized wells of a $0.8 \%$ agarose gel (without ethidium bromide stain), alongside the $\lambda$-HindIII marker, and run at $80 \mathrm{~V}$ for 2 hours in $1 \mathrm{x}$ TAE. The tank run buffer was changed to fresh buffer, and gel run overnight at $18 \mathrm{~V}$. The following morning, the marker lanes of the gel, and a portion of the eDNA containing sizes were excised from the gel, and post-stained in $0.5 \mu \mathrm{g} / \mathrm{mL}$ ethidium bromide TAE solution. Using a UV-transilluminator, the section of the gel corresponding to high molecular weight DNA (above the $23 \mathrm{~kb}$ marker band) was noted, and used as a guide to excise the portion of the gel that had not been exposed to ethidium bromide or UV light. The DNA in the resulting gel slice was recovered by electroelution, using a CB.S Scientific Electroeluter/concentrator ECU-040-20, running overnight at $100 \mathrm{~V}$ in $1 \mathrm{x}$ TAE buffer. The resulting elution was collected, and the concentrated using a 30,000 kDa molecular weight cut-off column centrifugal concentrator (Amicon), to a volume of $\sim 250 \mu \mathrm{L}$. This sample was then isopropanol precipitated, washed in $70 \%$ ethanol, and dry DNA pellet resuspended in $25 \mu \mathrm{L}$ TE for further processing (2.4.3).

\subsubsection{Preparation and transformation of competent cells}

Electrocompetent E. coli cells were routinely prepared from a number of strains (e.g. EC100, EPI300, S17-1). The desired strain was cultured overnight in 5mL LB broth at 37 ${ }^{\circ} \mathrm{C} / 200 \mathrm{rpm}$ for $12-16 \mathrm{~h} .10 \mathrm{~mL}$ of the overnight culture was then used to inoculate $500 \mathrm{~mL}$ LB medium in a baffled, sterile $2000 \mathrm{~mL}$ conical flask. The resulting culture was grown at $37{ }^{\circ} \mathrm{C} / 200 \mathrm{rpm}$ until an $\mathrm{OD}_{600}$ of $0.35-0.4$ was reached, at which point the culture was transferred into a sterile $750 \mathrm{~mL}$ centrifuge bottle and placed on ice for 15-30 min with occasional swirling. Cells were then collected by centrifugation $\left(3900 \mathrm{rcf}, 10 \mathrm{~min}, 4{ }^{\circ} \mathrm{C}\right.$ ), the supernatant discarded, and the pellet and bottle gently washed with sterile $\mathrm{ddH}_{2} \mathrm{O}$ without resuspension. The pellet was then resuspended in a total volume of $50 \mathrm{~mL}$ ice cold 
sterile $10 \% \mathrm{v} / \mathrm{v}$ glycerol, and transferred to a sterile $50 \mathrm{~mL}$ centrifuge tube. Cells were again pelleted by centrifugation (3900 $\mathrm{rcf}, 10 \mathrm{~min}, 4{ }^{\circ} \mathrm{C}$ ) and the resulting pellet resuspended $500 \mu \mathrm{L}$ ice cold sterile $10 \%$ v/v glycerol. $40 \mu \mathrm{L}$ aliquots were then distributed into pre-chilled $1.5 \mathrm{~mL}$ microfuge tubes on ice. Cell aliquots were snap-frozen using a metal tube block cooled to $-80{ }^{\circ} \mathrm{C}$. Frozen aliquots of cells were stored at $-80{ }^{\circ} \mathrm{C}$ until needed. When appropriate for plasmid maintenance, antibiotics were added to LB broth for growth steps.

Cell aliquots were removed from storage and placed on ice until thawed. Purified/desalted DNA/assembly was added to the cell aliquots to a volume not exceeding $10 \%$ of the aliquot. The tube was gently flicked to mix the contents and transferred to an ice cold, sterile $2 \mathrm{~mm}$ gap electroporation cuvette. Cells were then electroporated $(2.5 \mathrm{kV}, 25 \mu \mathrm{F}$, $100 \Omega$ ) using a Bio-Rad GenePulser Xcell, and $1 \mathrm{~mL}$ LB medium was immediately added with the cells gently resuspended and transferred to a sterile $15 \mathrm{~mL}$ tube. The cells were then incubated at $37{ }^{\circ} \mathrm{C} / 200 \mathrm{rpm}$ for $1 \mathrm{~h}$ for recovery, before plating on LB agar plates containing appropriate antibiotics, and incubated at $37{ }^{\circ} \mathrm{C}$ overnight for colony growth.

\subsubsection{DNA analysis}

\subsubsection{Agarose gel electrophoresis}

Agarose gels of various concentrations were used for analysis of DNA with different size distributions, ranging from $0.5 \%(\mathrm{w} / \mathrm{v})$ to $2 \%(\mathrm{w} / \mathrm{v})$. Lower concentrations of agarose were used for analysis of higher molecular weight DNA, while smaller DNA (screening PCR products) was performed with higher agarose concentration. All agarose gels were made in $1 \mathrm{x}$ TAE buffer (40 mM Tris-acetate, $1 \mathrm{mM}$ EDTA), and run submerged in 1x TAE buffer. Running conditions were also varied for different DNA analysis, with lower voltage $(80 \mathrm{~V})$ used for high molecular weight DNA, and higher voltage (up to $140 \mathrm{~V}$ ) for low molecular weight DNA.

\subsubsection{Nanodrop measurement of DNA concentration}

Miniprepped DNA was assessed for concentration and purity using a Thermoscientific NanoDrop ND-1000 ${ }^{\mathrm{TM}}$ spectrophotometer. 


\subsubsection{DNA sequencing}

Macrogen Inc. performed Sanger DNA sequencing, Illumina HiSeq 100 bp PE sequencing, and PacBio SMRT sequencing.

GeneWiz performed sequencing of individual cosmids using Illumina HiSeq 150 bp PE sequencing.

\subsection{Metagenome library construction}

Methods for extraction of environmental DNA (eDNA) and construction of the cosmid metagenome libraries was based on Brady $2007^{92}$. Detailed descriptions and alterations are described below.

\subsubsection{Environmental DNA extractions from soil}

Soil samples of at least $1 \mathrm{~kg}$ were collected, and contaminating material (i.e. sticks, stones, bark, bugs etc.) removed prior to further processing. A sample of $250 \mathrm{~g}$ was weighed into a $1 \mathrm{~L}$ centrifuge bottle, with $270 \mathrm{~mL}$ heated $\left(70{ }^{\circ} \mathrm{C}\right)$ lysis buffer $(100 \mathrm{mM}$ Tris- $\mathrm{HCl}, 100$ mM Na EDTA, $1.5 \mathrm{M} \mathrm{NaCl}, 1 \%$ (w/v) cetyl trimethyl ammonium bromide, $\mathrm{pH} 8.0$ ) added on top of the soil. This was mixed to ensure the soil thoroughly wetted, and returned to the water bath to reach $70{ }^{\circ} \mathrm{C}$. A volume of $30 \mathrm{~mL}$ heated $\left(70{ }^{\circ} \mathrm{C}\right) 20 \% \mathrm{SDS}(\mathrm{w} / \mathrm{v})$ was added to the centrifuge tube, inverted to mix, and returned to the water bath. The sample was incubated for 2 hours, with gentle inversion every 30 minutes. The sample was then rapidly cooled in an ice/water bucket for 30 minutes, with inversion once in this time. The soil and chilled precipitate was then separated from the clarified lysate by centrifugation at $4500 \mathrm{rcf}$ for 35 minutes at $4{ }^{\circ} \mathrm{C}$. The supernatant was recovered, volume measured, and returned to room temperature by brief incubation in the warm water bath. Isopropanol was added to $0.7 \mathrm{x}$ the supernatant volume, mixed by gentle inversion to precipitate the eDNA. Following a 30 minute room temperature incubation, the precipitated eDNA was collected by centrifugation at $4500 \mathrm{rcf}$ for 35 minutes at $4{ }^{\circ} \mathrm{C}$. The supernatant was discarded, and the eDNA pellet and centrifuge bottle was washed with $100 \mathrm{~mL}$ of ice cold $70 \%(\mathrm{v} / \mathrm{v})$ ethanol. The sample was again centrifuged at $4500 \mathrm{rcf}$ for 10 minutes at $4{ }^{\circ} \mathrm{C}$. The supernatant was discarded, and the bottle briefly centrifuged again to collect the remaining ethanol, which was removed by pipette. The eDNA pellet was briefly air dried (no more 
than 15 minutes). A minimum volume of TE buffer was added to cover the eDNA pellet, and this was left to slowly resuspend overnight at room temperature. The following morning, gentle swirling was employed to complete the resuspension, and the sample was transferred to a $15 \mathrm{~mL}$ centrifuge tube for storage. Some samples required additional TE, or brief incubation at $50{ }^{\circ} \mathrm{C}$ to fully resuspend the eDNA pellet.

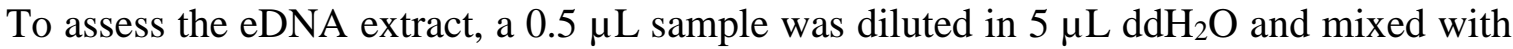
loading dye to $1.2 \mathrm{x}$ final concentration. This sample was run alongside a $\lambda$-HindIII marker on a $0.8 \%$ agarose gel at $100 \mathrm{~V}$ for 2 hours, and then post-stained overnight in $0.5 \mu \mathrm{g} / \mathrm{mL}$ ethidium bromide TAE solution prior to visualisation in a UV trans-illuminator.

\subsubsection{Cosmid packaging extract preparation}

The use of high-grade reagents appears to be critical for success in this protocol. The following suppliers were used for each reagent.

- Agar (Becton, Dickinson and Company (BD) Bacto ${ }^{\text {TM }}$ Agar (Ref 214010))

- Casein digest (Becton, Dickinson and Company (BD) Difco ${ }^{\mathrm{TM}}$ Casein Digest (Ref 211610))

- Yeast extract (Becton, Dickinson and Company (BD) Bacto ${ }^{\mathrm{TM}}$ Yeast Extract (Ref 212750))ATP, pH 7.0

- $\quad \beta$-mercaptoethanol (Sigma-Aldrich 2-Mercaptoethanol (M3148))

- EDTA (Sigma-Aldrich EDTA (EDS))

- Lysozyme (Sigma-Aldrich Lysozyme from chicken egg white (62970))

- $\mathrm{MgCl}_{2} .6 \mathrm{H}_{2} \mathrm{O}$ (Sigma-Aldrich Magnesiumchloride hexahydrate (M2670))

- Putrescine dihydrochloride (Sigma-Aldrich (P5780))

- Spermidine tryhydrochloride (Sigma-Aldrich (85578))

- Sucrose (Sigma-Aldrich (S7903))

- Tris-base (Sigma-Aldrich Trizma® Base BioXtra (T6791))

- ATP (Sigma-Aldrich Adenosine 5'-triphosphate disodium salt hydrate (A7699))

- $\mathrm{HCl}$ (Sigma-Aldrich HCL 36.5-38\% (H1758) but Univar Hydrochloric acid 36\% conc. Analytical Reagent grade worked as well)

- $\mathrm{NaOH}$ (to adjust $\mathrm{pH}$, may not need to be specific supplier, used Scharlau Sodium hydroxide pellets, Reagent Grade (SO 0425)) 
- Chloroform (does not need to be from a specific supplier)

Using the above reagents, the protocol described in Techniques in Aquatic Toxicology, Volume 2, chapter 38, appendix 1: Preparation of $\lambda$ packaging extracts (Winn and Norris 2010) ${ }^{113}$ was followed. Minor changes to the protocol were made to suit laboratory specific conditions.

\subsubsection{Cosmid library construction}

The eDNA substrate for cosmid library construction was first extracted from soil samples (section 2.4.1), and then size selected by agarose gel extraction and electroelution (section 2.3.4). The DNA sample was quantified by nanodrop (section 2.3.6.2), then end-repaired using End-It DNA End-Repair Kit (Epicentre) according to the manufacturer's instructions. The DNA was isopropanol precipitated to remove the end-repair enzymes, and resuspended in TE buffer.

The cosmid vector (pWEB or pWEB::tnc) was miniprepped (section 2.3.1), and digested with SmaI restriction enzyme (NEB), and dephosphorylated with Antarctic phosphatase (NEB) according to the manufacturer's instructions. The sample was heated to deactivate the enzymes, and the DNA isopropanol precipitated and resuspended in TE buffer.

The prepared cosmid vector (250 ng) and eDNA substrate (125 ng) were then ligated in a final reaction volume of $5 \mu \mathrm{L}$ using Fast-link DNA ligase (Epicentre) according to the manufacturer's instructions. One tube of each packaging extract strain were thawed quickly, and combined ( $40 \mu \mathrm{L} \mathrm{A}, 60 \mu \mathrm{L} \mathrm{B}$ ) to create the usable packaging extract. Ligated DNA was packaged by adding $12.5 \mu \mathrm{L}$ of packing extract per ligation reaction, incubation at $30{ }^{\circ} \mathrm{C}$ for 90 minutes, followed by the addition of a further $12.5 \mu \mathrm{L}$ packaging extract and incubation step. $250 \mu \mathrm{L}$ of phage dilution buffer $(10 \mathrm{mM}$ Tris-HCl pH 8.3, $100 \mathrm{mM}$ $\mathrm{NaCl}, 10 \mathrm{mM} \mathrm{MgCl}_{2}$ ) was then added, followed by $7 \mu \mathrm{L}$ chloroform. The sample was gently mixed, the chloroform pelleted by brief centrifugation, and the diluted phage heads added in a 1:10 ratio to an ice cold, day culture of E. coli EC100 (or EC100 $\Delta e n t D$ ) grown to $\mathrm{OD}_{600}$ of $1.0 \mathrm{in} \mathrm{LB} 10 \mathrm{mM} \mathrm{MgSO}_{4}$. The phage head/cell mixture was incubated at room temperature for 20 minutes, then aliquotted across 96 wells (if desired) and incubated at 37 ${ }^{\circ} \mathrm{C} 200 \mathrm{rpm}$ for 75 minutes. The recovered, transfected $E$. coli cells were then diluted in LB with appropriate antibiotics for cosmid selection, and titre samples of select wells plates 
plated on LB agar with antibiotics, for colony counting and library size estimations. The remaining cultures were incubated overnight at $37^{\circ} \mathrm{C} 200 \mathrm{rpm}$ to replicate the library clones in liquid culture. The following morning, samples were taken from each of the 96 aliquots to make glycerol stocks for long term storage, alongside minipreps of each library "well".

The input volumes for ligation and packaging reactions described above are the minimal usable volumes, and the reactions were scaled linearly as desired for larger cosmid library construction.

\subsection{Phosphopantetheinyl transferase library screening}

Prior to screening, metagenome libraries were divided into "screening pools" of various sizes. This is discussed further in Chapter 3.

Each screening pool was cultured overnight in LB with appropriate antibiotics from a glycerol stock. Overnight cultures were sub inoculated into fresh LB with antibiotics (100 $\mu \mathrm{L}$ into $3 \mathrm{~mL}$ fresh media) and cultured for an additional 3 hours. From the fresh culture, $1 \mathrm{~mL}$ was transferred to a sterile microcentrifuge tube, and the cells collected by centrifuging at top speed for 1 minute. The supernatant was discarded, and the cells resuspended in $1 \mathrm{~mL} 10 \%(\mathrm{v} / \mathrm{v})$ sterile glycerol. The cells were collected by centrifuge again, with this wash step repeated twice more. The cells were resuspended in $1 \mathrm{~mL} 10 \%$ glycerol and then a sample diluted 1/10 in 10\% (v/v) glycerol for $\mathrm{OD}_{600}$ measurement in a spectrophotometer. Using the formula $\mathrm{OD}_{600}$ of $1.0=1.24 \times 10^{8}$ cells $/ \mathrm{mL}$, the cells were diluted in $10 \%(\mathrm{v} / \mathrm{v})$ glycerol to a final concentration of $3 \times 10^{7}$ cells $/ \mathrm{mL}$. The first round of screening requires 6 million cells per screening plate, therefore $200 \mu \mathrm{L}$ of the diluted cell stock was spread over a single $150 \mathrm{~mm}$ diameter iron limited screening agar plate, which was then incubated at $37{ }^{\circ} \mathrm{C}$ for no more than 13 hours.

Following the incubation, the plates were monitored closely, and once small, translucent patches of colonies appeared on the agar surface, $2-3 \mathrm{~mL}$ of sterile $10 \%$ glycerol was added to the agar plate, and the surface scraped to suspend the colony cells in the liquid. This was then collected, and the cell stock washed in $10 \%$ glycerol three times as above. A washed cell sample was diluted into $10 \%$ glycerol for $\mathrm{OD}_{600}$ measurement as above, and then the stock diluted to a concentration of 50,000 cells per $\mathrm{mL}$. The second round of screening requires 10,000 cells per agar plate, therefore $200 \mu \mathrm{L}$ of this diluted cell stock 
was spread over another fresh $150 \mathrm{~mm}$ diameter iron limited screening agar plate. The plate was again incubated at $37^{\circ} \mathrm{C}$ for no more than 13 hours.

Once small colonies began to appear on the agar surface, they were picked onto another iron limited screening agar plate, and incubated overnight at $37{ }^{\circ} \mathrm{C}$. Restreak colonies that successfully grew were then transferred to LB broth with appropriate antibiotics, and cultured overnight, prior to preparation of glycerol stocks for long term storage.

\subsection{Transformation associated recombination (TAR) cloning}

The Kallifidias and Brady $2012{ }^{114}$ protocol was followed for the preparation of yeast spheroplasts, and for the transformation of the spheroplasts with pTARa capture vector and overlapping clones.

The major divergences from this protocol were as follows; S. cerevisiae BY4727 $\triangle$ NHEJ strain was used for spheroplast production, as the knocked out non-homologous end joining DNA repair mechanism reduced the "no-insert" cloning background rate considerably. The pTARa capture vector capture arms were designed in silico and ordered as synthetic DNA blocks, rather than generation by PCR. The cosmid overlap clones were pre-digested by in vitro CRISPR-Cas9, guided to the cosmid vector ends, rather than restriction endonuclease digestion.

The CRISPR guide RNAs target to the pWEB::tnc vector $~ 190 \mathrm{nt}$ upstream of the cosmid insert site (GGTTATTGTCTCATGAG), and 115 nt downstream of the cosmid insertion site (GTTAAATTGCTAACGCAGTC).

\subsection{Conjugation into Streptomyces expression strains}

Constructs to be conjugated into Streptomyces were first transformed by electroporation (see 2.3.5) into $E$. coli S17-1. Overnight cultures of the S17-1 "donor" strain were cultured with appropriate antibiotics in $5 \mathrm{~mL}$ LB broth overnight. The following day, the $0.5 \mathrm{~mL}$ of the overnight culture was sub inoculated into $10 \mathrm{~mL}$ of fresh $\mathrm{LB}$ with appropriate antibiotics, and cultured at $37{ }^{\circ} \mathrm{C}$ until $\mathrm{OD}_{600}$ reached $0.5-1.0 . \mathrm{S} 17-1$ cultures were then placed on ice for at least 10 minutes to halt growth, washed in fresh ice-cold LB broth twice to remove all antibiotics, and resuspended in a final volume of $1 \mathrm{~mL} \mathrm{LB}$. 
A volume of $\sim 5 \mu \mathrm{L}$ of the "recipient" $S$. albus strain spore stock was diluted into $0.5 \mathrm{~mL}$ TSB or LB, and heat shocked at $50{ }^{\circ} \mathrm{C}$ for 10 minutes to inactivate restriction endonucleases.

The donor and recipient stocks were then mixed in a 1:1 v/v ratio, and centrifuged at 4000 rcf for 5 minutes. The cell pellet was resuspended in $50-100 \mu \mathrm{L}$ of the residual $\mathrm{LB}$, and spread onto ISP4 agar, without antibiotics. Plates were incubated for $12-16$ hours at $30{ }^{\circ} \mathrm{C}$, and then flooded with $1 \mathrm{~mL}$ of the appropriate antibiotic stock mix (for pTARa $0.5 \mathrm{mg} / \mathrm{mL}$ nalidixic acid, $1.25 \mathrm{mg} / \mathrm{mL}$ apramycin sulfate, and for pIJ10257 $0.5 \mathrm{mg} / \mathrm{mL}$ nalidixic acid, $2.0 \mathrm{mg} / \mathrm{mL}$ hygromycin). Plates were incubated at $30{ }^{\circ} \mathrm{C}$ for $24-72$ hours until colonies emerged.

\subsection{Heterologous expression of biosynthetic gene clusters}

Starter cultures of $50 \mathrm{~mL}$ TSB were inoculated with $5 \mu \mathrm{L}$ of the desired Streptomyces spore stock, and cultured at $30{ }^{\circ} \mathrm{C}$ shaking for $24-48$ hours. Mature TSB cultures were then used to inoculate R5a expression culture at a $1 \%$ v/v concentration, and incubated at $30{ }^{\circ} \mathrm{C} 200$ rpm for 6 - 14 days as appropriate. R5a was at times supplemented with 20 g/L Superclo Dianon HP20 when desired for compound extraction.

\subsection{Small molecule analysis}

\subsubsection{Column chromatography}

All solvents used for column chromatography were of HPLC grade. Solvent mixtures are reported as \% v/v unless otherwise stated.

Reversed-phase column chromatography was achieved using Supelco Dianion ${ }^{\circledR}$ HP20 chromatographic resin.

Size exclusion chromatography employed Sephadex ${ }^{\circledR}$ LH20 resin.

Normal phase flash column chromatography used SiliaFlash F60 $(40-63 \mu \mathrm{m})$ silica as a column gel resin. 


\subsubsection{HPLC protocols}

An Agilent Technologies 1200 Series HPLC System was used. This system contained the following modules: 1200 series G1322A Degasser, G1311A Quaternary pump, G1329A autosampler, G1316A thermostatted column compartment, 1100 Series G1315B Diode array detector (DAD), and 1200 series G1364C analytical fraction collector

A generic HPLC protocol was used for most initial analyses of culture extract. This was a linear gradient from $10 \%$ to $100 \%$ acetonitrile $/ \mathrm{H}_{2} \mathrm{O}(0.1 \%$ acetic acid) over 25 minutes, with subsequent 7 minute hold at $100 \%$ acetonitrile ( $0.1 \%$ acetic acid). A NUCELODUR (® $\mathrm{C}_{18}$ HTec reversed-phase column was used (150 mm x $4.6 \mathrm{~mm}, 5 \mu \mathrm{m}$; Machery-Nagel), with $1.5 \mathrm{~mL} / \mathrm{min}$ flow rate and a column temperature of $40{ }^{\circ} \mathrm{C}$. DAD monitored $220 \mathrm{~nm}$, $252 \mathrm{~nm}, 280 \mathrm{~nm}, 320 \mathrm{~nm}, 380 \mathrm{~nm}$ wavelengths and spectrum absorbance from 200-800nm.

Bioactivity guided fractionation experiments employed several rounds of HPLC extract separation, in each case with shallower gradients. The analytical fraction collector module was employed to collect fractions at 1 minute time intervals for agar spot bioassay and MS analysis. Each of these experiments used Hypersil GOLD ${ }^{\mathrm{TM}} \mathrm{C}_{18}$ reversed-phase column ( $250 \mathrm{~mm} \times 4.6 \mathrm{~mm}, 5 \mu \mathrm{m}$; Thermo Scientific) with $1 \mathrm{~mL} / \mathrm{min}$ flow rate at a temperature of $40{ }^{\circ} \mathrm{C}$. The initial HPLC protocol used a gradient of $10-100 \%$ acetonitrile/ $\mathrm{H}_{2} \mathrm{O}(0.1 \%$ formic acid) over 35 minutes. The second round gradient was $40-70 \%$ acetonitrile/ $\mathrm{H}_{2} \mathrm{O}$ ( $0.1 \%$ formic acid) over 35 minutes. The third and final round employed a gradient of 30 $65 \%$ acetonitrile/ $\mathrm{H}_{2} \mathrm{O}(0.1 \%$ formic acid $)$ over 70 minutes.

\subsubsection{Mass spectrometry}

Mass spectrometry analysis was conducted on a Agilent 6530 Accurate Mass Q-TOF LCMS equipped with a 1260 Infinity binary pump.

In cases where a column chromatography was used upstream of the MS module, an InfinityLab Poroshell 120 EC-C 18 column was used $(100 \mathrm{~mm}$ x $2.1 \mathrm{~mm}, 2.7 \mu \mathrm{m}$; Agilent Technologies).

Agilent MassHunter Qualitative Analysis B.05.00 software was used for MS data analysis. 


\subsubsection{Nuclear Magnetic Resonance spectroscopy}

A $600 \mathrm{MHz}$ Varian Direct Drive spectrometer equipped with a 5 mm PFG dual broadband probe was used to record the NMR spectra.

MestReNova version 12.03-21384 software was used for NMR data analysis.

\subsection{Small molecule bioactivity assays}

For agar spot bioactivity assays, an overnight culture of B. subtilis 168 grown in LB medium was diluted $1 / 1000\left(\mathrm{v} / \mathrm{v}\right.$ ) in molten LB agar medium (cooled to $50{ }^{\circ} \mathrm{C}$ in a water bath). Agar plates were poured, set, and the surfaces briefly air dried. Test compounds or extracts were suspended in either methanol or DMSO, and 1-2 $\mu \mathrm{L}$ spotted onto the agar surface and allowed to absorb into the agar medium prior to overnight incubation at $30{ }^{\circ} \mathrm{C}$. Solvent only spots (methanol or DMSO) were used as a negative control. Results were assessed by a zone of clearance around the location of the spot.

Minimum inhibitory concentration (MIC) studies were performed using protocols derived from the Clinical and Laboratory Standards Institute (CLSI) and the European Committee on Antimicrobial Susceptibility Testing (EUCAST) standards ${ }^{115}$. The protocol in Wiegand et al. 2008 was followed to test isolated compounds against test strains of E. coli 7NT and B. subtilis 168. All tests were carried out in $\mathrm{MH}$ medium at $30{ }^{\circ} \mathrm{C}$, with 16 hour growth time in biological triplicate $(n=3$ independent experiments). Positive controls of tetracycline and mithramycin A were included in the analysis. All compounds were assayed from $128 \mu \mathrm{g} / \mathrm{mL}$ in two-fold serial dilution steps across 20 dilution stages, except for mithramycin, where the starting concentration was $32 \mu \mathrm{g} / \mathrm{mL}$. Sterility (media only) and growth (cells only) controls were included in each plate assayed.

The same protocol was followed for fungal MICs against $S$. cerevisiae $\Delta \mathrm{PDR}$, with the following changes. The growth medium used was YPD. The positive control antibiotic was changed from tetracycline to nystatin. The end-point measurement was changed from an eyesight measure to optical density, where a growth inhibited well was one where the OD measurement was $<90 \%$ of the growth control well (as per EUCAST guidelines for fungal susceptibility testing).

Antitumour assays were performed by Dr Abigail Sharrock using the following protocol, with positive control compound mithramycin. A standard $48 \mathrm{~h}$ MTS cell proliferation assay 
was used to evaluate cytotoxic activity against the human colon carcinoma cell line HCT116 ( $n=4$ independent experiments). Cells were treated with compound at various concentrations, and a dose-response was generated relative to a control of untreated HCT116 cells.

\subsection{Bioinformatic analysis}

Additional computational scripts are provided in Appendix A.

\subsubsection{Assembly of next generation sequencing data}

Illumina HiSeq data (whether 100 bp PE, or 150 bp PE reads), always passed through a pre-assembly QC and data curation pipeline. This pipeline was encoded in the decontam.sh shell script (written by fellow PhD student Mathew Storey, Appendix A).

PacBio raw reads QC statements were given by the sequence provider (Macrogen Inc.). The read files (*.fastq) were concatenated into a single .fastq file prior to assembly.

Assembly of sequencing data in Chapter 4 used hybrid-meta-SPAdes, with the following parameters:

module load spades/3.12.0

spades.py --meta --threads 40 --memory 200 -k 21,33,55,77 -

-pe1-1 1P.fq --pe1-2 2P.fq --pacbio PB.fastq -o

assemb1y. out

Sequencing data of cosmids in Chapters 5 and 6 did not involve "long" PacBio reads, and were thus assembled solely from "short" Illumina HiSeq PE reads, using the following parameters:

module load spades/3.12.0

spades . py --meta --threads 40 --memory 200 -k 21, 33, 55 , 77 --pe11 1 . fq--pe1-2 2P. fq-o assembly.out 


\subsubsection{Biosynthetic gene cluster analysis}

\subsubsection{Antibiotic and secondary metabolite analysis shell (antiSMASH)}

Nucleotide .fasta files were analysed using antiSMASH v.4.1.0 ${ }^{22}$, with the following parameters:

antismash in.fasta --clusterblast--subclusterblast --

knownclusterblast --smcogs--cpus 24 --outputfolder

antiSMASH.out

\subsubsection{Basic Local Alignment Search Tool (BLAST)}

Routine BLAST search was performed using the NCBI BLAST Command Line Application, using either the NCBI-nr database, or custom BLAST databases (e.g. searching against the MIBiG database protein sequences) using the makeblastdb command.

Larger BLAST jobs (large query sets searches against large databases in Chapter 4) were performed using DiamondBLAST ${ }^{116}$, as the search algorithm is orders of magnitude faster than standard BLAST.

diamond blastp - d /path/to/database - q /path/to/query.faa o output.txt --outfmt 6 --max-hsps 1 --max-target-seqs 1

DIAMOND was also used for taxonomy search of query sequences against the NCBI taxonomy database.

diamond blastp -d /path/to/taxa/database - q /path/to/query.faa -o output.txt --outfmt 102 --max-hsps 1 --max-target-seqs 1

\subsubsection{Biosynthetic gene similarity clustering and prospecting engine (BiG-SCAPE)}

BiG-SCAPE v0.0.0 117,118 was used to group biosynthetic gene clusters into similarity networks, running antiSMASH output as the input files. The accompanying CORASON pipeline was run alongside BiG-SCAPE from the same input commands.

source activate bigscape 
python BiG-SCAPE-master/bigscape.py -i /gbks --mode glocal --cutoffs $0.50 .6 \quad 0.7$--cores 36 --mix --include_singletons --mibig -o bigscape.out 


\subsection{Soil environmental DNA extraction and metagenome library construction}

\subsection{Summary}

Metagenomic approaches to natural product discovery can take many forms, most of which require construction of clonal libraries harbouring genomic fragments from uncultivated bacteria. This work focused on gene cluster discovery from soil microbiomes, which are among the most complex microbial communities known (on the order of 10,000 species per environment ${ }^{7,9,10}$ ). The complexity of soil communities precludes facile direct sequencing. The strategy chosen in this study was to create cosmid libraries of the soil eDNA, which were separated out into less complex pools. These pools can later be screened to enrich for cosmid clones containing relevant biosynthetic genes, further reducing complexity and allowing for direct DNA sequencing and subsequent functional studies.

In this chapter, 55 soil samples were gathered from across the South Island (Te Waiponamu) of Aoteroa New Zealand. Environmental DNA (eDNA) was extracted from each soil sample, and assessed for quality and purity. Top candidate soils by these metrics progressed to "cloning trials", to assess empirically the ease with which cosmid libraries could be produced from each sample. Four samples were then chosen for full scale eDNA extraction and cosmid library construction.

The resulting libraries were used in the research described in later chapters $(4,5$, and 6), where they were interrogated by both functional and sequence based screening for markers of natural product biosynthesis.

\subsection{Introduction}

Soil microbial environments have historically been a privileged source of biodiversity for the discovery of bioactive natural products ${ }^{2,69}$. The relative abundance of natural products discovered from soil microbes has been hypothesised to be a result of the large number of bacterial species, on the order of 10,000 per soil environment ${ }^{7,9,10}$, and the competition between those species for space and resources within those environments ${ }^{119}$. A common 
response to this competition is the production of bacterial toxins to reduce or halt the growth of competing species ${ }^{120}$.

While many natural products have been isolated from soil microbes historically, the New Zealand soil microbial complement has been relatively under studied. The biodiversity of New Zealand is unique compared when compared to the rest of the world, due in part to relative isolation of the islands since the separation from the Gondwana landmass $80 \mathrm{Ma}$ 121,122 . This isolation has resulted in a marked divergence in evolutionary outcomes for New Zealand macroflora and fauna. It is therefore possible that the microbial life in New Zealand may also be evolutionarily distinct from the other environments studied to date. This unexplored biodiversity will undoubtedly contain undiscovered biosynthetic diversity, which could be exploited for the development of future antibiotics.

It has been demonstrated that the biosynthetic genetic capacities of a soil's microbial complement changes with distance between sample sites, changes in latitude, and changes in biome ${ }^{14,15,88}$. The geographic isolation of New Zealand, and diversity of biomes in such a compact area make it an attractive area for natural product discovery efforts.

\subsection{Sample site selection}

Ideally, collected soil samples would be representative across each of the metrics of sample site biome, distance and latitude ${ }^{14,15,88}$. For practical reasons, sampling was restricted to one island of Aoteroa New Zealand. The South Island (Te Waiponamu) was selected over the North Island (Te Ika-a-Māui), as the shape and orientation of the island allowed a greater range of latitudes to be covered more quickly than with the North Island. The South Island also has a high diversity of soil types (Figure 3.1), significant variation in rainfall patterns from the West Coast to the East, and soil drainage properties that could impact microbial population composition ${ }^{123}$. Localised areas of soil diversity are also present in the South Island - e.g. the limestone base of Castle Hill Basin.

Soil samples were gathered on two separate sampling trips, with soils 1-13 gathered in August 2015, and samples 14-55 in February 2016. 


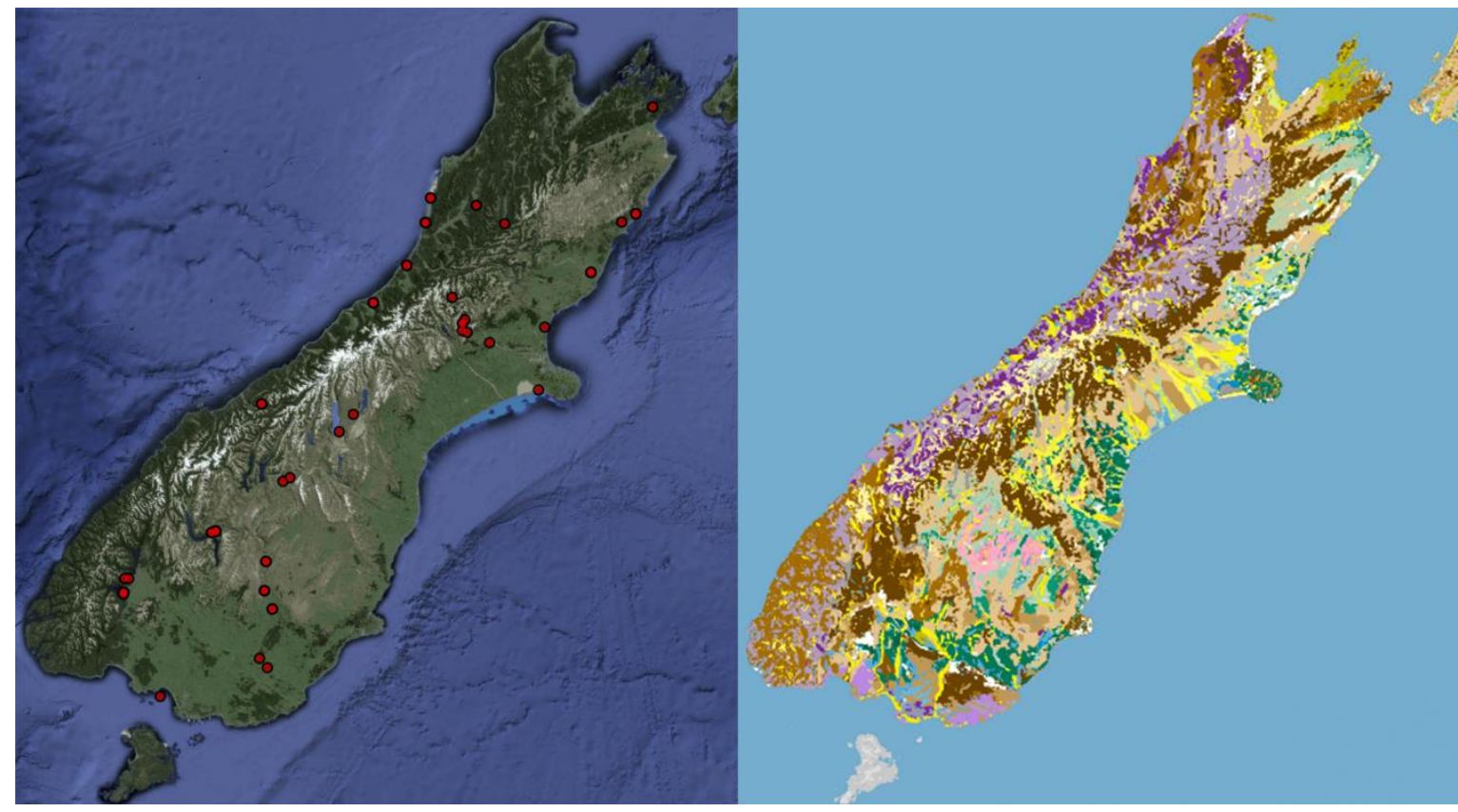

Figure 3.1 - Soil sample collection sites and soil classification map of New Zealand. The 55 soil sample collection sites are represented by red dots on the left hand map. Soil classification map (right) was generated on Manaaki Whenua Landcare Research Soils Map Viewer, with data sourced from Land Information New Zealand (CC BY-NC-ND 3.0 NZ). Soil classification map is coloured according to New Zealand Soil Classification Order (15 divisions) and Group (74 divisions) ${ }^{123}$.

\subsection{Environmental DNA extraction}

Initially, a published protocol for high molecular weight soil eDNA extraction (Brady 2007) was followed for each soil sample ${ }^{92}$. However, many of the soil eDNA extracts resulting from this protocol were found to be unsuitable for downstream analysis - often being too contaminated with salts and detergents for analysis via gel electrophoresis. After significant changes to the published protocol, useable eDNA extracts were recovered. The fully optimised protocol is provided in detail in Chapter 2. In brief, soil samples were weighed, and combined with a heated lysis buffer containing salts, detergents and buffers to lyse bacterial cells, neutralise humic acid contaminants and protect the free DNA. After two hours of lysis, the samples were cooled to precipitate excess salts, detergents and cellular components, prior to centrifugation to remove soil and precipitates. The DNA was recovered from the clarified lysate by isopropanol precipitation. The DNA pellets were washed, briefly dried and resuspended in TE buffer. All agarose gel electrophoresis was performed in 1x TAE rather than 0.5x TBE (as in Brady 2007), as boric acid inhibits downstream molecular biology processes. 
Following optimisation of the extraction protocol, a scaled down version was used to extract eDNA from small samples $(6-7 \mathrm{~g})$ of each soil, and the resulting eDNA extracts assessed to identify soils that were likely to yield DNA that was able to be efficiently cloned. The primary metrics by which the suitability of eDNA was examined before proceeding to cloning trials were amount of eDNA extracted, purity of eDNA, and the molecular weight of the eDNA (Figure 3.2). The amount of eDNA extracted per unit of soil was not so important, as most samples had $>2 \mathrm{~kg}$ of useable soil, which would yield sufficient DNA for library construction even for soil with low abundance of biomatter. Cosmid cloning requires DNA in the size range of 30-40 kb, so eDNA extracts without useable amounts of DNA in this size range cannot be used for library construction. DNA with low purity (i.e. high degree of soil contaminants including humic acid species) is difficult to clone as the contaminants strongly inhibit the downstream enzymatic processing of DNA (end repair, ligations, phage packaging).

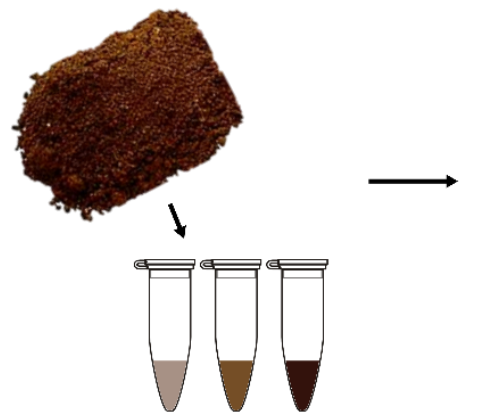

Soil eDNA is extracted, with rough grading based on extract colour, darker colour indicating more sample contamination.

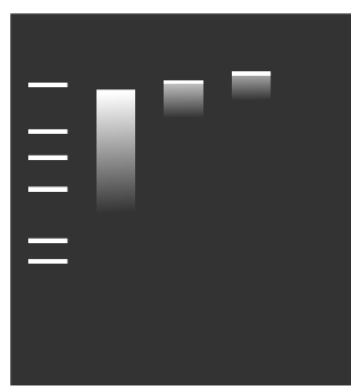

Agarose gel based assessment of eDNA, high quality samples have band above $23 \mathrm{~kb}$ marker and minimal smearing.

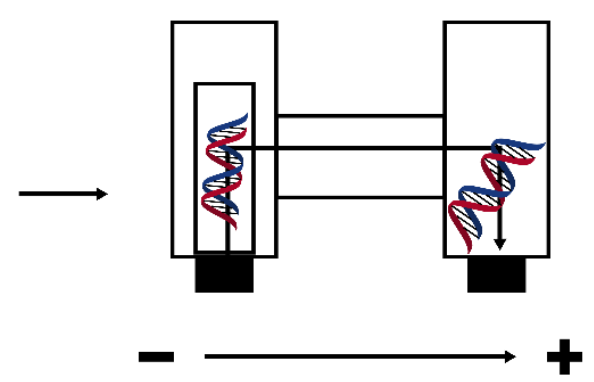

Gel extraction of high molecular weight eDNA from gel slices using electroelution, where DNA migrates from gel slice and is collected in adjacent chamber.

Figure 3.2 - Environmental DNA extraction and grading process. Once the eDNA had been extracted from the soil samples, the initial gating occurred whereby samples were graded for apparent purity (colour of sample) and appearance by agarose gel electrophoresis. Samples with reasonable purity and high molecular weight DNA were taken forward for size selection and electroelution from agarose gel slice. The purified, size selected eDNA samples then proceeded to cosmid cloning trials.

The primary metric for assessing eDNA samples was by agarose gel electrophoresis (Figure 3.2). The molecular weight distribution of the extracted DNA was assessed by running a $15 \mu \mathrm{L}$ sample on a $0.8 \%$ agarose gel (Figure 3.3 and Figure 3.4). In standard agarose gel electrophoresis, DNA does not migrate linearly as a function of molecular weight - high 
molecular weight DNA tends to cluster and co-migrate through the gel. To assess the presence of suitably high molecular weight DNA for use in cosmid library construction, a DNA band above the $23 \mathrm{~kb}$ marker band of a $\lambda$-HindIII digest was considered to be a positive result. Colour of the eDNA sample was also used as a secondary metric for the degree of soil contamination in the eDNA sample, however final decisions on which soils to proceed with were made based on agarose gel results, as most contamination could be removed during the downstream size selection and electroelution steps.

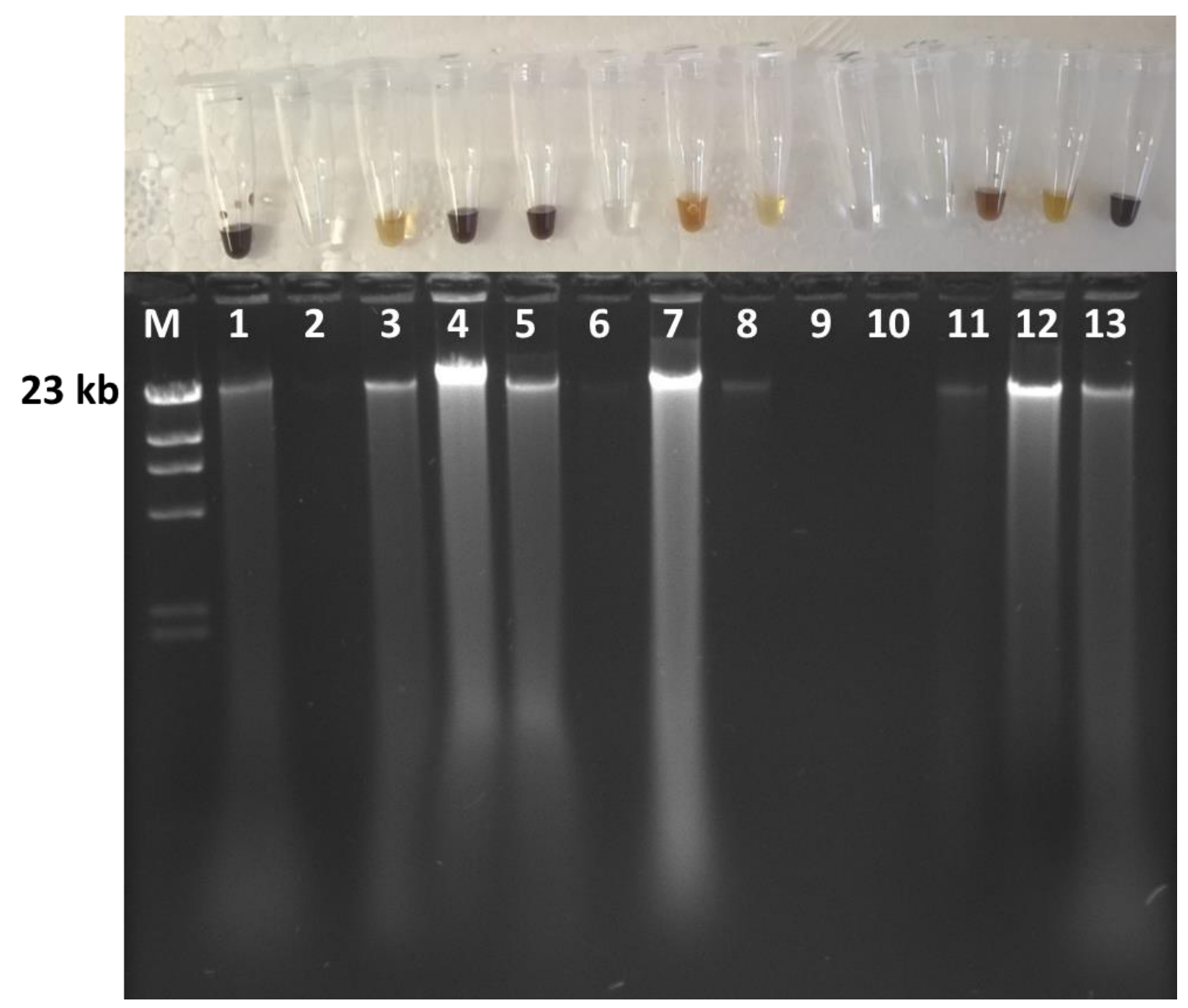

Figure 3.3 - Extracts and agarose gel of environmental DNA extractions from soils 1-13. Upper panel shows the variation in appearance of eDNA extractions. Lower panel shows the results of agarose gel electrophoresis of these eDNA extracts. The $\lambda$-HindIII marker is used to assess whether the molecular weight of the DNA is suitable for cosmid cloning, with DNA above the top marker band (23kb) indicating a positive result. 


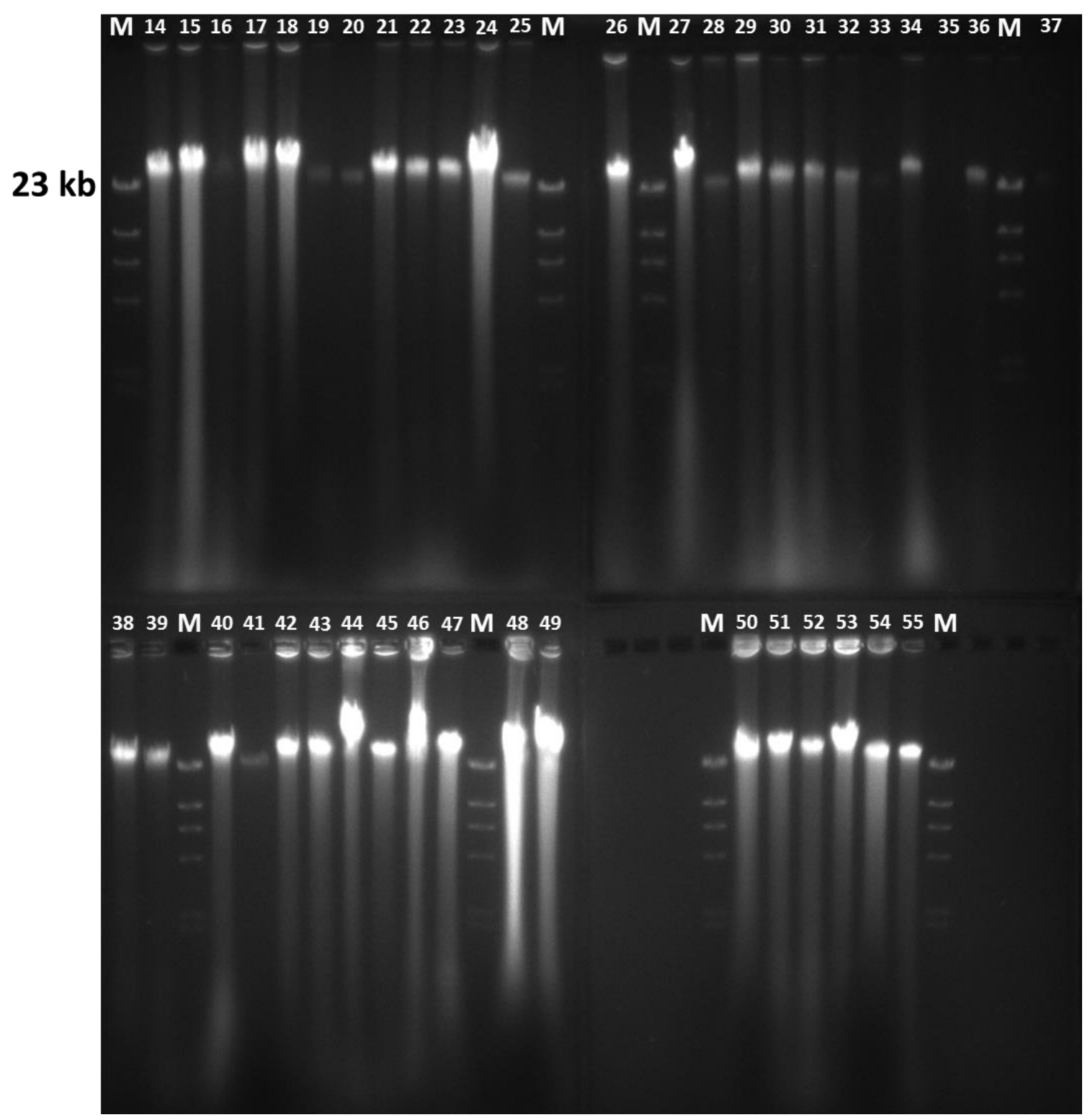

Figure 3.4 - Agarose gel of environmental DNA extractions from soils 14-55. Some samples yielded very high amounts of eDNA, thus overloading the gel causing uneven banding (e.g. sample 24). Other soils appeared to contain auto-fluorescing impurities, which interfered with DNA visualisation (e.g. sample 48).

Based on this qualitative scoring system, soil samples $3,4,7,8,12,14,17,28,34,40,44$, $46,52,53$, and 55 were taken forward for large scale extractions and cloning efficiency trials. Larger scale extractions were performed on $250 \mathrm{~g}$ of each selected soil, and the resulting extracts were again primarily graded by the results of agarose gel electrophoresis.

The large scale eDNA extracts were then further processed prior to any enzymatic reactions. This was achieved by size selection agarose gel electrophoresis, where an agarose gel was run overnight at low voltage $(20 \mathrm{~V})$, to achieve separation of high 
molecular weight DNA from both low molecular weight DNA and soil contaminants. The high molecular weight eDNA was excised from the gel, and the DNA recovered by electroelution from the gel slice, followed by concentration in a $30,000 \mathrm{kDa}$ molecular weight cut off column and isopropanol precipitation of the DNA (Figure 3.2).

\subsection{Library construction trials}

In order to redundantly cover the majority of microbial genomes in a soil, approximately 10 million cosmid clones are required ${ }^{111}$. Construction of libraries of this size is significantly easier when high quality DNA that clones with high efficiency is used. To evaluate the suitability of each eDNA sample for cosmid library preparation, the series of reactions required for library construction were performed in small scale. Construction of large soil metagenome libraries is a time consuming and expensive process, therefore only the eDNA samples most amenable to library construction (i.e. those that will require the fewest packaging reactions) were carried forward into full scale library construction.

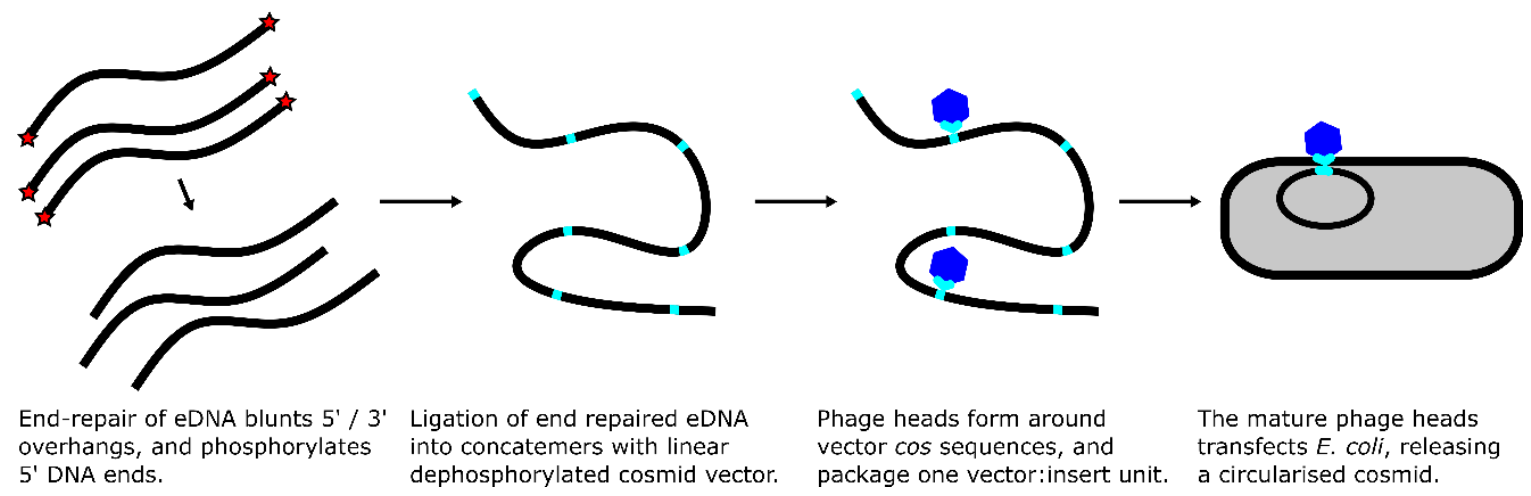

Figure 3.5 - Cosmid cloning scheme. The isolated eDNA is initially end-repaired to blunt and phosphorylate the DNA fragment ends, followed by ligation in vector:eDNA concatemers with dephosphorylated cosmid vector. The cos sequences on the cosmid vectors provide attachment points for the $\lambda$-phage heads to assemble around a single vector:insert DNA unit, which the mature phage head transfects into $E$. coli to create a circular cosmid.

The downstream ligation reaction in cosmid cloning requires the eDNA insert to have blunt ends and 5'-phosphorylation. Our extraction process generates eDNA samples which have been randomly fragmented throughout the extraction processes, and an end-repair step was therefore necessary to generate cloning competent DNA. End repair was carried out using 
the End-It DNA End-Repair Kit (Epicentre), which removes overhanging 5'/3' nucleotide overhangs and carries out 5' phosphorylation. This kit contains T4 DNA polymerase, which fills in 5' overhangs with 5 ' $\rightarrow 3^{\prime}$ ' polymerase functionality, and removes 3 ' overhangs with 3' $\rightarrow 5^{\prime}$ exonuclease activity. T4 DNA polynucleotide kinase phosphorylates 5' ends of the blunted DNA molecules.

The cosmid DNA vector for cloning trials (and library construction) of eDNA samples 113 was commercially prepared pWEB, provided in the MaxPlax cosmid cloning kit (Epicentre). For subsequent cloning trials of samples 14-55, the pWEB::tnc vector was used, and was prepared in house. pWEB::tnc is a preferable cosmid cloning vector, as its shorter vector sequence (pWEB 8179bp, pWEB::tnc 5812bp) permits a larger eDNA insert size in cosmid cloning, where the final construct is size limited by phage packaging restrictions. pWEB::tnc empty vector was miniprepped from an E. coli EC100 strain, digested with SmaI restriction endonuclease (blunt end cut), and dephosphorylated using Antarctic phosphatase enzyme to remove 5' phosphates from the blunt DNA ends.

Ligation reactions using T4 DNA ligase combine the blunt, dephosphorylated vector sequence with the blunt phosphorylated insert sequence, linking the two substrates in insert:vector concatemers (Figure 3.5). The opposite phosphorylation patterns of the two DNA substrates result in ligation of long concatemers of alternating units. The addition of MaxPlax phage packaging extract (Epicentre) results in the formation of mature $\lambda$-phage heads where each contain a single vector/insert unit, which forms a complete cosmid when transfected to E. coli cells.

This process was carried out for each soil where large scale eDNA extraction was performed, and the cloning trial results of the pWEB::tnc reactions (soils 14-55) is summarised below in Table 3.1. A single packaging reaction was performed for each sample, and dilutions of the resulting E. coli cell suspension plated out on LB agar supplemented with ampicillin and chloramphenicol to select for cosmid containing cells. The number of colonies resulting from each dilution were used to calculate the cosmids that could be constructed in a single packaging reaction for each eDNA sample. From this result, the number of packaging reactions that would be required to construct a full 10 million member library was calculated. 


\begin{tabular}{|c|c|c|c|c|c|c|}
\hline $\begin{array}{l}\text { Soil } \\
\text { eDNA } \\
\text { sample }\end{array}$ & $\begin{array}{l}\text { Undiluted } \\
\text { transfection }\end{array}$ & $\begin{array}{l}\text { 1/10 } \\
\text { dilution }\end{array}$ & $\begin{array}{l}\text { 1/100 } \\
\text { dilution }\end{array}$ & $\begin{array}{l}\text { Colonies in } \\
110 u \mathrm{~L} \\
\text { adhered cells }\end{array}$ & $\begin{array}{l}\text { Cosmids in } \\
\text { one packaging } \\
\text { reaction }\end{array}$ & $\begin{array}{l}\text { Packaging } \\
\text { reactions for } 10 \\
\text { million clones }\end{array}$ \\
\hline 14 & 36 & 5 & 0 & 110 & 3080 & 3246.8 \\
\hline 16 & TMTC & 41 & 6 & 902 & 25256 & 395.9 \\
\hline 17 & TMTC & 41 & 5 & 902 & 25256 & 395.9 \\
\hline 18 & TMTC & 55 & 5 & 1210 & 33880 & 295.2 \\
\hline 24 & TMTC & 96 & 8 & 2112 & 59136 & 169.1 \\
\hline 38 & TMTC & 91 & 11 & 2002 & 56056 & 178.4 \\
\hline 40 & TMTC & 14 & 1 & 308 & 8624 & 1159.6 \\
\hline 43 & TMTC & 26 & 2 & 572 & 16016 & 624.4 \\
\hline 49 & TMTC & 91 & 6 & 2002 & 56056 & 178.4 \\
\hline 50 & TMTC & 44 & 5 & 968 & 27104 & 369.0 \\
\hline 53 & TMTC & 136 & 18 & 2992 & 83776 & 119.4 \\
\hline 55 & TMTC & 55 & 6 & 1210 & 33880 & 295.2 \\
\hline Control & TMTC & TMTC & 93 & 20460 & 572880 & 17.5 \\
\hline
\end{tabular}

Table 3.1 - Library construction trial results for pWEB::tnc libraries. For each soil eDNA sample, a single packaging reaction was completed, and $10 \mu \mathrm{L}$ of packaged phage heads was transfected into $100 \mu \mathrm{L}$ of a mature E. coli EC100 culture, recovered and dilutions of $50 \mu \mathrm{L}$ volume plated onto LB agar with ampicillin and chloramphenicol for cosmid selection. Resulting colony counts were used to assess cloning efficiency and predict the reaction required for library construction. Control DNA insert was provided in the MaxPlax cosmid cloning kit (Epicentre). TMTC = "too many to count".

The results showed a large degree of variation in the cloning efficiencies of the different soil eDNA preparations. Although soil 24 had high cloning efficiency, the large scale eDNA extract had very low yield of eDNA, and the sample became too limiting to construct a library. From the cloning trial results, the four soil samples predicted to require the fewest packaging reactions to produce a 10 million clone library were selected; soil 4 (Castle Hill Basin/CH), soil 38 (Roxburgh/RX), soil 49 (Saint Anne's Lagoon/SL), and soil 53 (Half Moon Bay/HM). These soils are also reasonably evenly geographically distributed across the South Island, and cover a range of soil environments, providing conditions for distinct microbial communities in each soil chosen (Figure 3.6). 


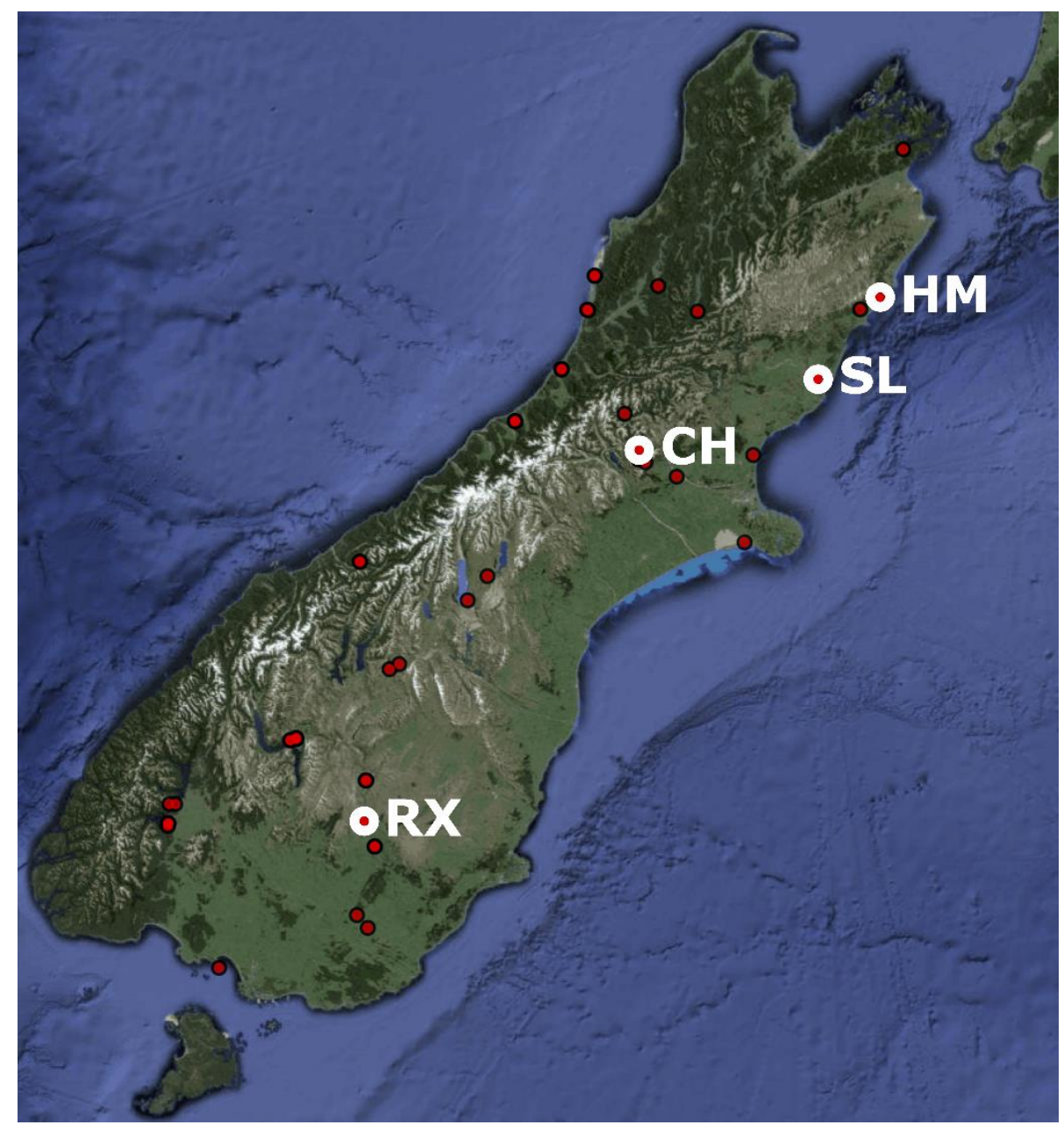

Figure 3.6 - Map of soil sample collection sites around the South Island of New Zealand. All soil sample sites indicated by a red dot, with the soil samples used in the construction of large eDNA libraries indicated by white circles Castle Hill Basin (CH), Half Moon Bay (HM), St. Anne's Lagoon (SL), Roxburgh (RX).

\subsection{Production of packing extract}

During the course of the library construction process, commercial supplies of phage packaging extract became unavailable. In order to complete the partially constructed cosmid metagenome libraries, a new source of packaging extract was required. In collaboration with postdoctoral researcher Mark Calcott, we were able to produce the packaging extract ourselves, using a modified version of the protocol from Winn and Norris $2005^{113}$.

Cloning trial results indicated that the "in-house" produced packaging extract had a cloning efficiency greater than the two previously available commercial kits (Agilent SuperCos and Epicentre MaxPlax packing extracts), summarised in Table 3.2. The three packaging 
extracts were used to construct small cosmid libraries from three DNA sources; Roxburgh soil eDNA (RX), Saint Anne's Lagoon soil eDNA (SL), and a control insert DNA sample (Control) that was included with the MaxPlax cosmid cloning kit (Epicentre). Packaging reactions were carried out under standard conditions, adhered to E. coli EC100 cells, and dilutions of the recovered cells plated out onto LB agar supplemented with ampicillin and chloramphenicol. The colonies that grew from each packaging reaction were counted, and used to measure the packaging efficiency of each extract.

\begin{tabular}{lllll}
\hline Substrate DNA & Packaging extract & $\begin{array}{l}\text { Undiluted } \\
\text { transfection }\end{array}$ & $\mathbf{1 / 1 0}$ dilution & $\mathbf{1 / 1 0 0}$ dilution \\
\hline RX & Agilent SuperCos & TMTC & 127 & 14 \\
RX & Epicentre MaxPlax & TMTC & 136 & 14 \\
RX & "In-house" & TMTC & 358 & 36 \\
\hline SL & Agilent SuperCos & TMTC & 165 & 23 \\
SL & Epicentre MaxPlax & TMTC & 162 & 16 \\
SL & "In-house” & TMTC & 479 & 42 \\
\hline Control & Agilent SuperCos & TMTC & TMTC & 74 \\
Control & Epicentre MaxPlax & TMTC & TMTC & TMTC \\
Control & "In-house" & TMTC & TMTC & TMTC \\
\hline
\end{tabular}

Table 3.2 - Packaging extract tests. Identical cosmid packaging reactions were set up on three different template DNA sources (metagenomic eDNA from Roxburgh soil (RX) and St. Anne's Lagoon (SL), and the control DNA from the Epicentre cosmid cloning kit). These different DNA substrates were then packaged in using three different packaging extracts, two commercial (Agilent SuperCos, Epicentre MaxPlax) and an "In house" prepared extract. Dilutions of each resulting packaging reaction adhered to E. coli $\mathrm{EC} 100$ cells were plated on LB agar with ampicillin and chloramphenicol for selection, and the colonies counted after 16 hours incubation. TMTC $=$ too many to count.

The control DNA substrate produced too many colonies to get an accurate measure of packaging efficiency. The two soil samples however, both had higher packaging efficiency in the "in-house" produced packaging extract than in either commercial extract, by a factor of 2-3. The packaging efficiency of the "in-house" produced packaging extract was then verified by culture of several colonies from the agar plates, and extracting the cosmids they contained. Restriction analysis of the resulting cosmid preparations confirmed the presence of suitably sized complete cosmid clones, each with a unique digested banding pattern (and therefore a unique DNA insert) in Figure 3.7. 


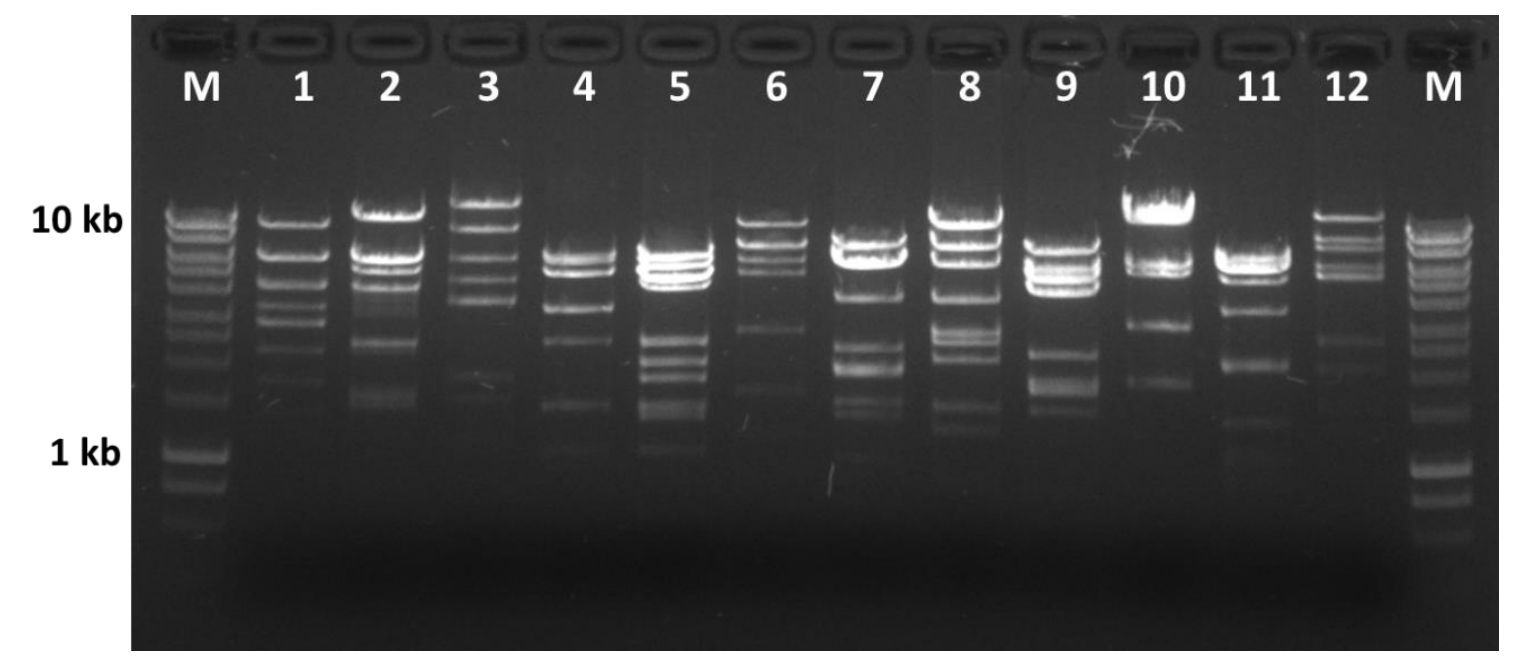

Figure 3.7 - Restriction analysis of twelve constructed cosmids. Maker lanes with Hyperladder I (Bioline) is used for reference. All cosmids were digested with HindIII and BamHI in combination, and present with different banding patterns when separated by agarose gel electrophoresis. The banding patterns and size distributions indicate all cosmids contain unique metagenome inserts.

\subsection{Construction of four soil metagenome cosmid libraries}

For large scale library construction, multiple packaging reactions were combined into a single tube, adhered to E. coli cells (EC100 for CH library/EC100 $\Delta e n t D$ HM, RX and SL libraries), and then arrayed in 96 well plate format, where each pool contained clonal copies of 2,000 - 46,000 unique cosmid clones (Table 3.3). An arrayed library is more useful for screening purposes, where different wells, or pools of wells can be screened independently, rather than the library as a whole. This can result in greater sensitivity and depth of screening. A visual representation of the arrayed library is provided in Figure 3.8. Each of the four library plates was separated evenly across 96 wells and then cultured overnight for replication of each unique cosmid. The following morning, wells were then independently pooled by rows (i.e. a subsample of wells 1-12 combined into pool "R1"). A subset of each of the row pools was then combined into a "mega-pool" (MP) that contains a representation of all of the cosmids contained within that cosmid library plate. The three levels of complexity for each library plate (well, row pool and mega pool) can be used for screening/sequencing where different levels of DNA complexity are desired. 


\section{HM-A}
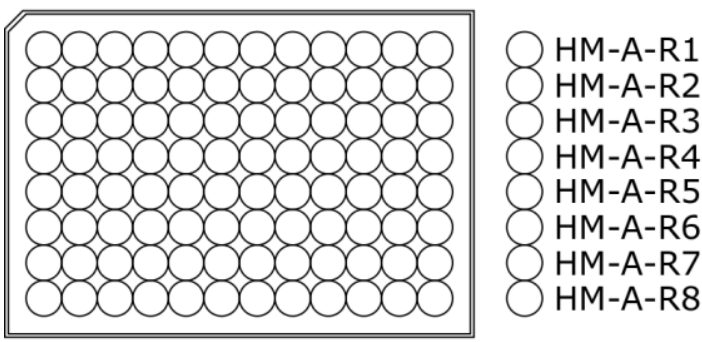

HM-A-MP

\section{$\mathrm{HM}-\mathrm{C}$}
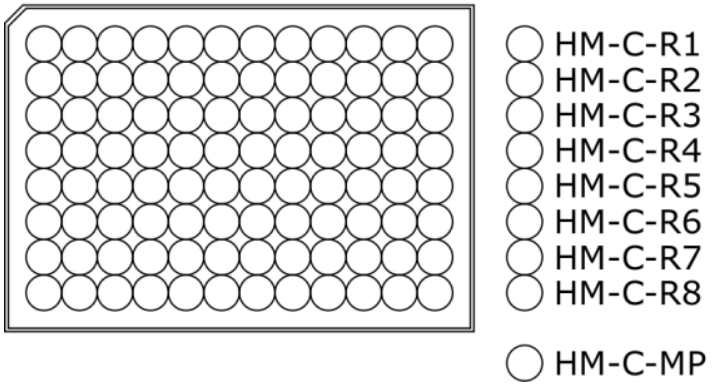

HM-B

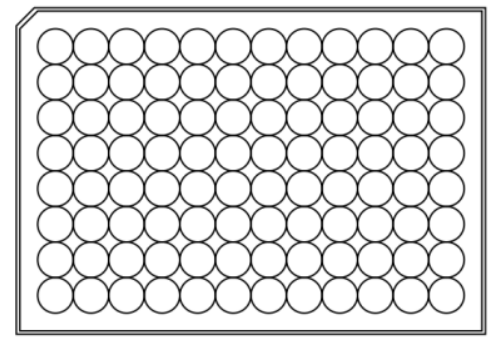

$H M-B-R 1$

$\mathrm{HM}-\mathrm{B}-\mathrm{R} 2$

$\mathrm{HM}-\mathrm{B}-\mathrm{R} 3$

HM-B-R4

HM-B-R5

HM-B-R6

HM-B-R7

HM-B-R8

HM-B-MP
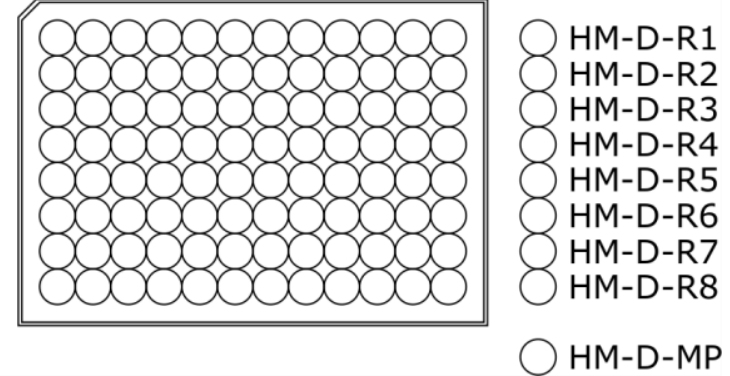

Figure 3.8 - Arrayed metagenome library from Half Moon Bay soil eDNA. Prior to replication, each library plate was aliquoted across 96 wells, to give a plate of 96 independent subsets of equal complexity (number of unique cosmids). After replication (overnight incubation) subsets of wells are combined into "row pools" (R1-8), where each row pool contains a representative of all of the cosmids contained in each parent well. Row pools for each plate are then combined into "mega pools" (MP), where each mega pool contained a representative of all of the cosmids in the library plate it represents.

To estimate the number of cosmid clones in each library plate, "titre" experiments were conducted during the construction process. In these experiments, a small sample of recovered cells $(50 \mu \mathrm{L}$ from each of three $5 \mathrm{~mL}$ wells) were spread on LB agar supplemented with antibiotics, and the remaining sample was grown to confluence and used in glycerol stock preparations. The resulting number of colonies on each titre plate was used to calculate the number of unique cosmid clones in the corresponding well. In general, each 96 well plate was constructed independently, on different days, with the number of packaging reactions used per plate being informed by the results of previous days. The library construction efficiency at the large scale was dramatically improved over the small-scale trials; for example, HM library (soil 53) was estimated from the small-scale trial to require 119 packaging reactions, while the 10.6 million clone library was eventually created using 46.5 packaging reactions. The improvement for the RX and SL libraries that were created with the homemade packaging extract was even more dramatic (due in part to 
the use of the "in-house" prepared packaging extract). This may have been a result of the greater accuracy and efficiency when following a single, large-scale preparation protocol, rather than many small-scale reactions in parallel.

Details of the final constructed libraries are summarised in Table 3.3 below, including the host strain of $E$. coli, the cosmid vector used, and the number of cosmid clones in each library plate and overall library sizes.

\begin{tabular}{lllll} 
& $\begin{array}{l}\text { Castle Hill } \\
\text { Basin }(\boldsymbol{C H})\end{array}$ & $\begin{array}{l}\text { Half Moon } \\
\text { Bay }(\boldsymbol{H M})\end{array}$ & $\begin{array}{l}\text { Roxburgh } \\
(\boldsymbol{R X})\end{array}$ & $\begin{array}{l}\text { Saint Anne's } \\
\text { Lagoon }(\boldsymbol{S L})\end{array}$ \\
\hline Host strain & EC100 & EC100 $\Delta$ entD & EC100 $\Delta e n t D$ & EC100 $\Delta e n t D$ \\
\hline Cosmid Vector & pWEB & pWEB::tnc & pWEB::tnc & pWEB::tnc \\
\hline Plate A & 384,000 & $1,230,000$ & $1,750,000$ & 370,000 \\
Plate B & 384,000 & $4,170,000$ & $1,080,000$ & $1,130,000$ \\
Plate C & 768,000 & $2,950,000$ & $3,570,000$ & $4,440,000$ \\
Plate D & 768,000 & $2,200,000$ & $2,090,000$ & $2,900,000$ \\
Plate E & 768,000 & - & 200,000 & $4,430,000$ \\
Plate F & 768,000 & - & - & - \\
Plate G & 313,0000 & - & - & - \\
Plate H & 313,0000 & - & - & - \\
Plate I & 291,0000 & - & - & - \\
Plate J & 291,0000 & - & - & $13,270,000$ \\
\hline Total library size & $15,920,000$ & $10,550,000$ & $8,690,000$ & \\
\hline
\end{tabular}

Table 3.3 - Cosmid metagenome libraries constructed in this study. Number of unique clones per library plate "mega pool" and total library complement are provided, along with E. coli host strain and cosmid vector.

\subsection{Discussion}

In this chapter, four large insert cosmid libraries were constructed from soil eDNA metagenome samples. These soil samples were collected across the South Island of New Zealand, taking into account geographic spacing and the biomes of the collected samples. However, upon beginning the construction of these libraries we soon discovered that the majority of the DNA sequences contained in the eDNA extracts were highly novel and divergent from the genetic information contained in online sequence databases. Because of this, the primary metric for selection of soils to use became cloning efficiency, and soil 
sample geography became a secondary concern. Nonetheless, the four soil samples used in full-scale cosmid metagenome library construction had reasonably evenly distributed origins across the South Island.

Significant optimisation of all protocols involved in eDNA isolation and library construction was required. Initial soil eDNA extraction attempts failed due to multiple missing steps in published protocols - including the correct composition of extraction buffers and critical sample cooling/heating steps, as well as suboptimal buffer composition for agarose gel extractions. Updated protocols have been provided in Chapter 2. The precipitation of buffer components can be avoided by combining preheated lysis buffer (made without SDS) with soil samples, and reheating before separately adding hot SDS solution, improving eDNA extraction dramatically. By cooling samples in an ice bucket post-lysis and prior to centrifuging out soil matter, salts and detergents co-pellet with soil, improving purity of the final product, as charged salts and detergents will inhibit DNA migration in agarose gel electrophoresis. Overnight drying of eDNA pellets as described in Brady $2007^{92}$ results in DNA pellets that cannot be resuspended - a quick dry procedure was followed instead. Published protocols instruct that eDNA purification should be performed in $0.5 x$ TBE agarose gel, however the boric acid in TBE buffer can inhibit downstream enzymatic reactions, and is very difficult to dissociate from DNA. 1x TAE was used instead to greater success.

Making the packaging extract in-house also required significant optimisation. Initial attempts all failed, due to the use of standard grade reagents. When high grade reagents for all media, buffers and enzymes in the process were used, the packing extract production was successful. The protocol with all regent specifications has been provided in Chapter 2 . This optimised protocol produced packaging extracts that were more efficient in cosmid clone production than the extract from commercial providers. The final products of the protocol are proteins used in the formation of phage capsids. These proteins are very sensitive to environmental conditions, and it is possible that the samples purchased from commercial suppliers may degrade in quality somewhat during potentially long transport time to New Zealand. Fresh samples produced in the lab and immediately used are likely to produce more optimal results, as demonstrated in section 3.6.

The four large libraries described in this chapter are a valuable resource, representing a preserved, renewable genetic snapshot of the soil microbial complement. In total, these 
libraries contain nearly 50 million cosmids, each carrying $30-40 \mathrm{~kb}$ of metagenome DNA. This is a collection of DNA information on the order of $1 \times 10^{12}$ bases, or a terabase of genetic data. As the median bacterial genome is $3.65 \mathrm{Mb}^{124}$, this is the raw data value equivalent of almost half a million bacterial genomes. The true representation of bacterial genome information is far less than this however, as each of the four soils likely contain around 10,000 bacterial species ${ }^{7,9,10}$, and these species are not likely equally weighted in either their genome size, population share in the soil, or their amenability to the lysis and cloning procedures followed in the course of this chapter. In addition, many of the metagenome insert sequences, though unique will contain "overlapping" genetic information. This is however, a desirable outcome that is exploited in the study described in Chapter 5 to piece together a biosynthetic gene cluster from multiple metagenome cosmid sequences. The intent behind making libraries of such a large size was to oversample, and gain high levels of coverage for each sequence in the library. Nevertheless, the amount of genomic diversity contained within these libraries is great, and if properly interrogated could lead to many discoveries of value to science and industry.

These libraries are the source material for all experimental work described in the remainder of this thesis (Chapters 4, 5, and 6). Other laboratory group members have also used the libraries for their own investigations into natural product metabolism and enzyme discovery. 


\subsection{Functional screening of soil metagenome libraries and bioinformatic analysis}

\subsection{Summary}

Phosphopantetheinyl transferase (PPTase) activity is a required posttranslational modification for many families of biosynthetic gene clusters (including NRPS, PKS, and hybrid biosynthesis systems). PPTases can be promiscuous, and activate carrier protein/thiolation domains outside of their native substrate. In the research described in this chapter, a PPTase knockout E. coli strain was used (E. coli $\Delta e n t D)$ to screen the four soil metagenome libraries described in Chapter 3. In this screen, libraries are enriched for cosmid clones carrying PPTase genes that are able to be expressed in E. coli and activate the enterobactin biosynthesis pathway, rescuing the screening strain when placed under iron starvation. This powerful enrichment tool focuses on NRPS and PKS biosynthetic gene clusters, to allow an economical use of DNA sequencing resources.

The resulting cosmid pools were then sequenced using a combination of Illumina HiSeq $100 \mathrm{bp}$ PE reads and PacBio sequencing platforms, to achieve the largest ever metagenome survey of mega-synth(et)ase gene clusters. The hybrid assembly DNA sequences of this dataset were then passed through the antiSMASH, BiG-SCAPE, CORASON, and custom analysis pipelines, to first annotate the presence of (partial) biosynthetic gene clusters in the recovered cosmids, and then identify related gene clusters and draw conclusions about the presence and uniqueness of soil metagenome biosynthetic gene clusters relative to those known in cultured genome databases. The primary question of this study was "Is the secondary metabolic profile of soil microbiomes effectively captured in the subset of bacteria that are readily cultivable?"

\subsection{Introduction}

Metagenome libraries should by design contain the (near) complete genetic complement of the organisms in the environmental sample used to construct the library. The four soil metagenome libraries described in Chapter 3 were made using an eDNA extraction protocol optimised for bacterial DNA extraction. This study focused on biosynthetic gene clusters 
involved in secondary metabolite production, which makes up on average $0.6 \%$ of a bacterial genome ${ }^{125}$, with estimates ranging from $2 \%$ to up to $5-10 \%$ amongst the "biosynthetically gifted" actinomycete phyla ${ }^{125,126}$. In order to retrieve the rare cosmids that contain secondary metabolic genes, a PPTase selection strategy was employed.

As previously discussed, PPTase enzymes are required for activation of NRPS and PKS biosynthetic systems (sections 1.2, 1.3, 1.8.2). The genes for PPTase enzymes are often located within NRPS and PKS biosynthetic gene clusters, where they serve to activate the thiolation domains of the megasynth(et)ase enzymes. PPTase screening has previously been employed to retrieve natural product biosynthesis genes from metagenomic libraries 99,101. In this study, the $\Delta e n t D$ iron-limiting selection strategy described in Charlop-Powers et al. 2013 was used ${ }^{101}$.

In iron-limiting conditions, many bacteria use siderophore compounds, which are exported from the cell, sequester iron from the environment, and transport the iron back into the cell. Laboratory E. coli strains typically possess genes for the biosynthesis of the siderophore enterobactin, which is produced by an NRPS biosynthesis system (Figure 4.1). The enterobactin biosynthesis pathway requires activation by the PPTase EntD to attach phosphopantetheine arms to the T-domains of EntB and EntF before substrate monomers 2,3-dihydroxybenzoic acid (DHB) and L-serine can be recruited, linked to form DHB-Ser, and cyclised with two other DHB-Ser molecules to form enterobactin (Figure 4.1). When the entD gene is absent, $E$. coli can only produce enterobactin if another active PPTase gene is present within the cell that can activate the T-domains of EntB and EntF. As enterobactin is required for survival under iron-limiting conditions, this knockout strain of E. coli (EC100 $\Delta e n t D)$ can be used to screen cosmid metagenome libraries for cosmid clones containing PPTase genes, and consequently, the biosynthetic gene clusters that they are often linked to. 


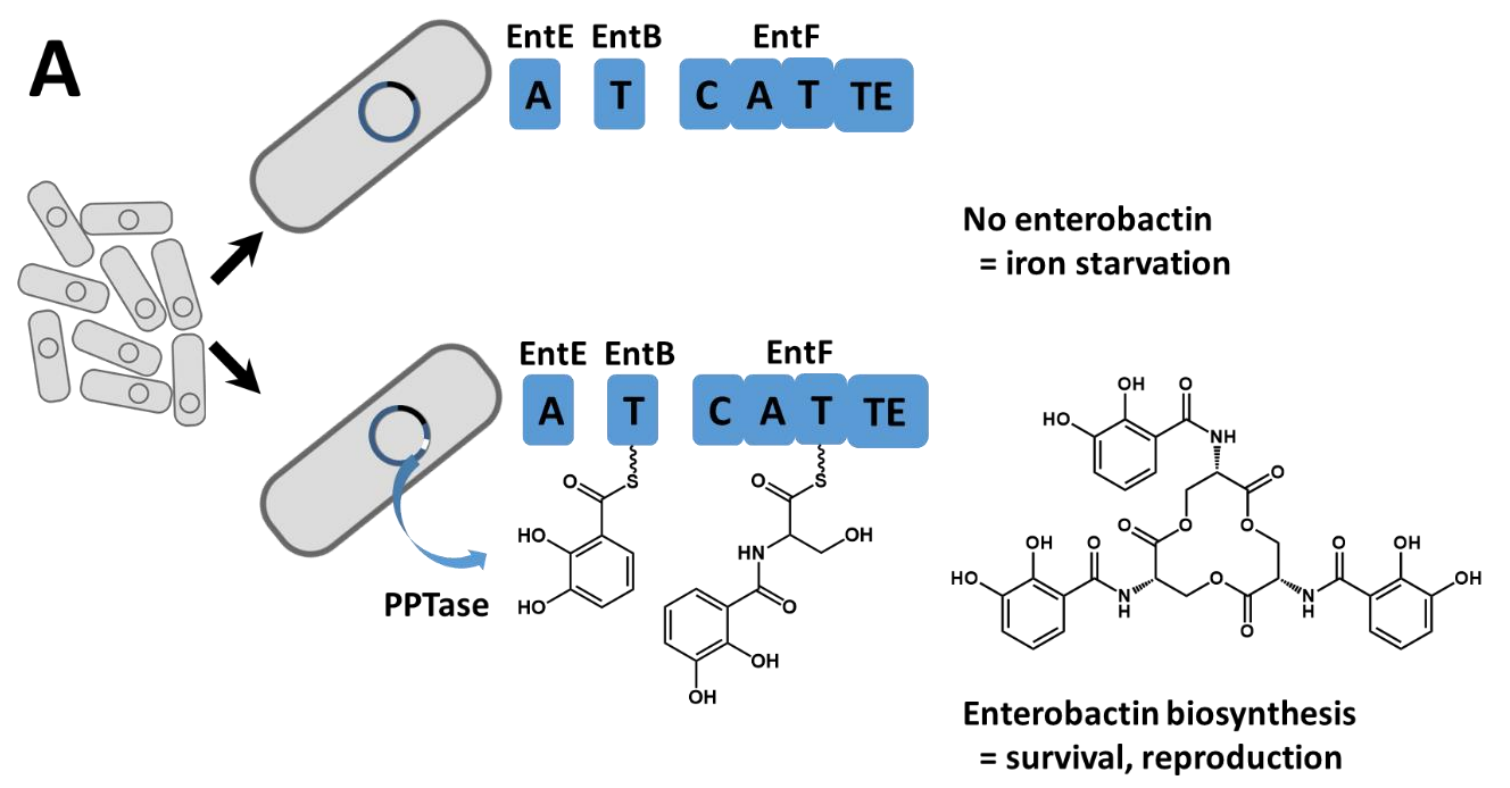

\section{B}

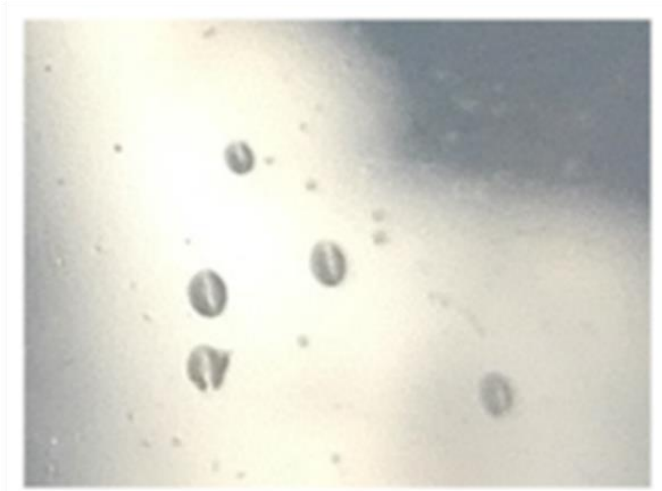

Figure 4.1 - Enterobactin PPTase screening strategy. A) In a pool of E. coli $\triangle e n t D$ cells each containing a cosmid from a metagenome library, those without a PPTase gene (upper) do not possess the ability to activate enterobactin biosynthesis, and thus cannot reproduce in iron-limiting conditions. However, when a PPTase gene is present (lower), the T-domains of EntB and EntF can be activated, permitting biosynthesis of enterobactin and allowing the E. coli $\Delta$ entD cells to sequester sufficient iron for survival and reproduction. B) Large colonies shown are "hit" colonies of this screening process, with the ability to produce enterobactin and therefore rapidly grow on iron-limited media. Smaller satellite colonies surround the larger colonies, surviving by taking up the enterobactin produced by the larger colonies.

\subsection{Small scale screening of the Castle Hill metagenome library}

Initially, pilot $\Delta e n t D$ screening was performed on the Castle Hill metagenome cosmid library. Screening occurred concurrently with the Castle Hill library construction in E. coli strain EC100 (Chapter 3). This necessitated the transfer of the cosmid library from the original EC100 strain into the knockout EC100 $\Delta e n t D$ strain to facilitate iron limited selection of PPTase gene containing cosmids. During this process, I discovered that transferring such large constructs from a library of $\sim 16$ million clones reliably with the 
required over-coverage was intractable. I found cosmids had a transformation efficiency $\sim 2$ logs less than plasmids, and to be confident I was screening the full library I would have liked to achieve $>10$-fold coverage of transformed cosmids into the test strain. In addition to this, reports in the literature suggest that extended cultivation of libraries biases the content, and therefore the results of a screen ${ }^{127}$. Retransformation was therefore concluded, in the long run, to be both intractable and not desirable. For this reason, the three later metagenome libraries (HM, RX, SL) were constructed directly in the EC100 $\Delta$ entD screening strain (Chapter 3). The Castle Hill library screening results were used as a "pilot study" - the results of which justified the effort of constructing three further libraries, and the data was fed into later analysis.

Transformation at this small scale was performed using $1 \mu \mathrm{g}$ of cosmid DNA from each of the $\mathrm{CH}$ library plate pools, each containing between 384,000 and 3.13 million unique clones, into EC100 $\Delta e n t D$ electrocompetent cells. These transformations gave results on the order of $1 \times 10^{5}-1 \times 10^{6}$ transformants per library plate, which translates to coverage of $0.05-0.85$ fold depending on the pool. Ideally $>10$-fold coverage would be achieved to give some assurance that most cosmids existing within the library had transferred to the screening strain. As we had decided at this point to construct future libraries in the test strain directly, these existing transformation pools were used to test the screening protocol and gather a preliminary dataset.

The screening protocol used is detailed in Chapter 2, and is a slightly modified version of the protocol in Charlop-Powers et al. $2013^{101}$. In brief, cultures of the library pools in EC100 $\Delta e n t D$ test strain were grown in LB + antibiotics overnight (for pWEB libraries; ampicillin and kanamycin). Cultures were then washed and diluted in $10 \%$ glycerol to remove media nutrients, and so that 6 million cells could be added to each $150 \mathrm{~mm}$ diameter iron-limited selection agar plate. Agar plates were incubated at $37{ }^{\circ} \mathrm{C}$ for no more than 13 hours to limit the growth of "satellite colonies" that quickly emerge by scavenging the enterobactin produced by "hit colonies" to facilitate their own growth (Figure 4.1B). The resulting colonies were scraped from the agar surface en masse, washed again, and plated at a lower titre of 10,000 cells per selection plate, and again incubated at $37{ }^{\circ} \mathrm{C}$ for no more than 13 hours. The emergent colonies were individually picked (with care taken to avoid satellite colonies) onto selection media one last time, before being transferred to LB media to prepare storage stocks and cosmid preparation for DNA sequencing. 
Key alterations in the protocol, when compared to that from Charlop-Powers et al. 2013, included: changing the starting source from a titered glycerol cell stock to an $\mathrm{OD}_{600}$ calibrated clean cell suspension, increasing the initial plating density to 6 million cells per plate, eliminating the intermediate growth phase in LB, and the use of $10 \%$ glycerol as a wash solution. The primary aim of implementing these changes was to increase the diversity of recovered sequences by avoiding outgrowth steps that might bias the enrichment. The modifications also facilitated the screening of many pools in parallel, eliminated unnecessary and time-consuming steps, and optimised the protocol for lab specific conditions.

In total, 491 colonies from the $\mathrm{CH}$ library were picked from this screening process. Prior to expensive next-generation sequencing of these cosmids, a small validation of the sequencing experiment was performed on 48 isolated colonies. These colonies were miniprepped, and the resulting cosmids Sanger sequenced from the outer edges of the metagenome inserts they contained (sequencing primers T7promoter and M13F-pUC). The sequence data was quality trimmed and analysed by BLASTx to align to the nearest homologue protein sequence on National Center for Biotechnology Information nonredundant protein sequences database (NCBI-nr) database (Table 4.1).

The results summarised in Table 4.1 show that 39/48 cosmids (81\%) align to a protein sequence potentially implicated in natural product biosynthesis or resistance. It is important to note that this sequencing only provides information on $\sim 1 \mathrm{~kb}$ of DNA sequence at each end of the cosmid insert sequence (i.e. $~ 6 \%$ of the full metagenome insert), and a negative result here does not necessarily indicate a lack of biosynthetic gene cluster on the sequenced cosmid. The data clearly demonstrate an enrichment for cosmids containing genes linked to secondary metabolism/biosynthetic gene clusters.

In addition to the presence of biosynthesis linked genes, this data shows that out of 48 cosmids, 32 were unique. The overrepresentation of some cosmids (cosmid 4 occurred 11 times, cosmid 27 occurred 3 times, and cosmids 6, 26, and 28 each occurred twice) could be a result of the "bottlenecking" of the cosmid population that occurred when transforming the cosmid pools into the screening strain of E. coli, and the extended culture/recovery time that results from this process. 


\begin{tabular}{|c|c|c|c|c|c|c|}
\hline & T7promoter alignment & $\begin{array}{l}\% \\
\text { identity }\end{array}$ & M13F-pUC alignment & $\begin{array}{l}\% \\
\text { identity }\end{array}$ & $\begin{array}{l}\text { Potential } \\
\text { NP function } \\
1\end{array}$ & $\begin{array}{l}\text { Potential } \\
\text { NP function } \\
2\end{array}$ \\
\hline 1 & serine protease & 48.05 & No result & & Resistance & \\
\hline 2 & $\begin{array}{l}\text { 4'-phosphopantetheinyl } \\
\text { transferase }\end{array}$ & 43.3 & MBOAT family protein & 48.75 & Biosynthesis & \\
\hline 3 & $\begin{array}{l}\text { cyclic peptide export } \mathrm{ABC} \\
\text { transporter }\end{array}$ & 72.84 & PAS domain S-box protein & 71.24 & Drug-efflux & \\
\hline 4 & $\begin{array}{l}\text { cyclic peptide export } \mathrm{ABC} \\
\text { transporter }\end{array}$ & 73.78 & PAS domain S-box protein & 71.24 & Drug-efflux & \\
\hline 5 & beta-lactamase TEM-1 & 88.75 & tetracycline resistance protein & 97.06 & $\begin{array}{l}\text { Antibiotic } \\
\text { resistance }\end{array}$ & \\
\hline 6 & $\mathrm{ABC}$ transporter permease & 47.22 & non-ribosomal peptide synthetase & 56.13 & Drug-efflux & Biosynthesis \\
\hline 7 & $\begin{array}{l}\text { cyclic peptide export } \mathrm{ABC} \\
\text { transporter }\end{array}$ & 72.84 & PAS domain S-box protein & 71.24 & Drug-efflux & \\
\hline 8 & $\begin{array}{l}\text { cyclic peptide export } \mathrm{ABC} \\
\text { transporter }\end{array}$ & 72.84 & PAS domain S-box protein & 71.24 & Drug-efflux & \\
\hline 9 & $\begin{array}{l}\text { cyclic peptide export ABC } \\
\text { transporter }\end{array}$ & 72.84 & PAS domain S-box protein & 71.24 & Drug-efflux & \\
\hline 10 & MBL fold metallo-hydrolase & 51.14 & bacterioferritin & 73.28 & $\begin{array}{l}\text { Antibiotic } \\
\text { resistance }\end{array}$ & \\
\hline 11 & methyltransferase & 37.18 & Hypothetical protein & & Biosynthesis & \\
\hline 12 & $\begin{array}{l}\text { cyclic peptide export } \mathrm{ABC} \\
\text { transporter }\end{array}$ & 73.62 & PAS domain S-box protein & 71 & Drug-efflux & \\
\hline 13 & $\begin{array}{l}\text { cyclic peptide export ABC } \\
\text { transporter }\end{array}$ & 73.78 & PAS domain S-box protein & 71.24 & Drug-efflux & \\
\hline 14 & $\mathrm{ABC}$ transporter permease & 43.12 & non-ribosomal peptide synthetase & 55.45 & Drug-efflux & Biosynthesis \\
\hline 15 & $\begin{array}{l}\text { cyclic peptide export } \mathrm{ABC} \\
\text { transporter }\end{array}$ & 73.78 & PAS domain S-box protein & 71.24 & Drug-efflux & \\
\hline 16 & $\begin{array}{l}\text { non-ribosomal peptide } \\
\text { synthetase }\end{array}$ & 60.66 & $\begin{array}{l}\text { efflux RND transporter } \\
\text { periplasmic adaptor subunit }\end{array}$ & 56.95 & Biosynthesis & Drug-efflux \\
\hline 17 & $\begin{array}{l}\text { cyclic peptide export } \mathrm{ABC} \\
\text { transporter }\end{array}$ & 72.84 & PAS domain S-box protein & 70.47 & Drug-efflux & \\
\hline 18 & $\begin{array}{l}\text { non-ribosomal peptide } \\
\text { synthetase }\end{array}$ & 90.18 & SDR family oxidoreductase & 92.47 & Biosynthesis & \\
\hline 19 & $\begin{array}{l}\text { cyclic peptide export } \mathrm{ABC} \\
\text { transporter }\end{array}$ & 72.84 & PAS domain S-box protein & 70.87 & Drug-efflux & \\
\hline 20 & $\begin{array}{l}\text { cyclic peptide export } \mathrm{ABC} \\
\text { transporter }\end{array}$ & 73.08 & PAS domain S-box protein & 70.87 & Drug-efflux & \\
\hline & No result & & metallophosphoesterase & 31.89 & & \\
\hline 21 & & & & & & \\
\hline 22 & $\begin{array}{l}\text { type II secretion system } \\
\text { protein }\end{array}$ & 46.31 & non-ribosomal peptide synthetase & 56.32 & Drug-efflux & Biosynthesis \\
\hline 23 & Hypothetical protein & & No result & & & \\
\hline 24 & $\begin{array}{l}\text { beta-ketoacyl-ACP synthase } \\
\text { I }\end{array}$ & 98.61 & $\begin{array}{l}\text { ribonucleoside-diphosphate } \\
\text { reductase subunit alpha }\end{array}$ & 98.85 & Biosynthesis & \\
\hline 25 & alanine--tRNA ligase & 90.21 & $\begin{array}{l}\text { DUF1501 domain-containing } \\
\text { protein }\end{array}$ & 74.86 & & \\
\hline 26 & $\begin{array}{l}\text { efflux RND transporter } \\
\text { periplasmic adaptor subunit }\end{array}$ & 69.83 & $\begin{array}{l}\text { acyl-[ACP]--phospholipid O- } \\
\text { acyltransferase }\end{array}$ & 63.73 & Drug-efflux & Biosynthesis \\
\hline 27 & ornithine cyclodeaminase & 83.85 & $\begin{array}{l}\text { uroporphyrin-III C- } \\
\text { methyltransferase }\end{array}$ & 57.83 & $\begin{array}{l}\text { Precursor } \\
\text { biosynthesis }\end{array}$ & Biosynthesis \\
\hline 28 & $\begin{array}{l}\text { 2,3-diaminopropionate } \\
\text { biosynthesis protein } \mathrm{SbnB}\end{array}$ & 83.33 & $\begin{array}{l}\text { uroporphyrin-III C- } \\
\text { methyltransferase }\end{array}$ & 68.82 & $\begin{array}{l}\text { Precursor } \\
\text { biosynthesis }\end{array}$ & Biosynthesis \\
\hline 29 & $\begin{array}{l}\text { 2,3-diaminopropionate } \\
\text { biosynthesis protein SbnB }\end{array}$ & 83.33 & $\begin{array}{l}\text { uroporphyrin-III C- } \\
\text { methyltransferase }\end{array}$ & 58.33 & $\begin{array}{l}\text { Precursor } \\
\text { biosynthesis }\end{array}$ & Biosynthesis \\
\hline 30 & DedA family protein & 55.1 & S9 family peptidase & 65.96 & Drug-efflux & $\begin{array}{l}\text { Antibiotic } \\
\text { resistance }\end{array}$ \\
\hline 31 & $\begin{array}{l}\text { NADH-quinone } \\
\text { oxidoreductase subunit } \mathrm{C}\end{array}$ & 41.28 & $\begin{array}{l}\text { DUF3488 domain-containing } \\
\text { protein }\end{array}$ & 57.38 & & \\
\hline 32 & $\begin{array}{l}\text { conserved membrane protein } \\
\text { of unknown function }\end{array}$ & 41.3 & S9 family peptidase & 73.24 & & $\begin{array}{l}\text { Antibiotic } \\
\text { resistance }\end{array}$ \\
\hline 33 & No result & & HPr kinase & 41.34 & & \\
\hline 34 & $\begin{array}{l}\text { non-ribosomal peptide } \\
\text { synthetase }\end{array}$ & 50 & HPr kinase & 41.11 & Biosynthesis & \\
\hline 35 & $\begin{array}{l}\text { 4'-phosphopantetheinyl } \\
\text { transferase }\end{array}$ & 46.64 & $\begin{array}{l}\text { MarR family transcriptional } \\
\text { regulator }\end{array}$ & 35.81 & Biosynthesis & \\
\hline 36 & $\begin{array}{l}\text { HAMP domain-containing } \\
\text { protein }\end{array}$ & 99.08 & cysteine synthase CysM & 92.8 & & $\begin{array}{l}\text { Precursor } \\
\text { biosynthesis }\end{array}$ \\
\hline 37 & No result & & phage DNA packaging protein & 48.15 & & \\
\hline 38 & ornithine cyclodeaminase & 83.75 & $\begin{array}{l}\text { uroporphyrin-III C- } \\
\text { methyltransferase }\end{array}$ & 89.36 & $\begin{array}{l}\text { Precursor } \\
\text { biosynthesis }\end{array}$ & Biosynthesis \\
\hline 39 & beta-lactamase TEM-1 & 56.17 & No result & & $\begin{array}{l}\text { Antibiotic } \\
\text { resistance }\end{array}$ & \\
\hline
\end{tabular}




\begin{tabular}{|c|c|c|c|c|c|}
\hline $\begin{array}{l}\text { phosphoenolpyruvate } \\
\text { synthase }\end{array}$ & 95.21 & $\begin{array}{l}\text { 4'-phosphopantetheinyl } \\
\text { transferase }\end{array}$ & 40.82 & $\begin{array}{l}\text { Precursor } \\
\text { biosynthesis }\end{array}$ & Biosynthesis \\
\hline No result & & DUF3187 family protein & 65.26 & & \\
\hline $\begin{array}{l}\text { HAMP domain-containing } \\
\text { protein }\end{array}$ & 93.81 & cysteine synthase CysM & 99.11 & & $\begin{array}{l}\text { Precursor } \\
\text { biosynthesis }\end{array}$ \\
\hline ornithine cyclodeaminase & 83.85 & $\begin{array}{l}\text { uroporphyrin-III C- } \\
\text { methyltransferase }\end{array}$ & 58.94 & $\begin{array}{l}\text { Precursor } \\
\text { biosynthesis }\end{array}$ & Biosynthesis \\
\hline glycosyltransferase & 31.13 & DNA packaging protein & 77.91 & Biosynthesis & \\
\hline $\begin{array}{l}\text { serine/threonine protein } \\
\text { kinase }\end{array}$ & 43.86 & alkaline phosphatase & 40.54 & & \\
\hline $\begin{array}{l}\text { 2,3-diaminopropionate } \\
\text { biosynthesis protein } \mathrm{SbnB}\end{array}$ & 82.24 & No result & & $\begin{array}{l}\text { Precursor } \\
\text { biosynthesis }\end{array}$ & \\
\hline $\begin{array}{l}\text { NADH-quinone } \\
\text { oxidoreductase subunit C }\end{array}$ & 41.28 & $\begin{array}{l}\text { DUF3488 domain-containing } \\
\text { protein }\end{array}$ & 59.3 & & \\
\hline $\begin{array}{l}\text { efflux RND transporter } \\
\text { periplasmic adaptor subunit }\end{array}$ & 67.37 & $\begin{array}{l}\text { acyl-[ACP]--phospholipid O- } \\
\text { acyltransferase }\end{array}$ & 62.26 & Drug-efflux & Biosynthesis \\
\hline
\end{tabular}

Table 4.1 - End-sequencing of cosmids from $\Delta e n t D$ screened CH library. Sanger sequencing data was trimmed and a BLASTx search performed against the NCBI-nr database, with percent identity to result in the adjacent column. "No result" indicates no alignment returned from BLASTx search against NCBI-nr database. The BLASTx alignment results were then annotated, where the protein could be conceivably involved in a natural product biosynthesis pathway.

Of the colonies that were picked from the screening plates of the $\mathrm{CH}$ library, 263 were cultured and pooled together ( $\mathrm{CH} \Delta e n t D$ pool), and the cosmids isolated for next generation sequencing using both the PacBio SMRT cell and Illumina HiSeq 2500100 bp PE platforms. Only around half of the cosmids that were picked were sent for sequencing, due to concerns around sequencing depth being too low to get the required coverage for full cosmid sequence assembly. Upon assembly of the sequence data, this was found not to be an issue, and the later sequencing runs for the remaining three libraries were performed with the full set of screened cosmids in each instance. NGS sequence data assembly is discussed in detail in section 4.5.

\subsection{Large scale screening and sequencing of the remaining three soil metagenome libraries}

Having verified that the screening procedure was capable of rapidly enriching for biosynthetic systems, three further cosmid libraries were constructed directly in the EC100 $\Delta e n t D$ screening strain of E. coli (see Chapter 3), and screened by the same process. Most bacteria contain only one Sfp-type PPTase per genome ${ }^{101,128}$, and the median bacterial genome size is $3.65 \mathrm{Mb}^{124}$. Estimates for soil metagenome complexity range from 1,00010,000 unique species per sample, therefore, each 10 million clone library should contain between 1,000-10,000 unique PPTase genes covered many times. However, other factors severely limit the number of clones likely to pass through the selection gating; genes must 
be able to be transcribed from native promoter sequences in the E. coli host, transcripts must translate in spite of codon bias, and the resulting PPTase enzyme must both fold correctly and function with the T-domain substrate of both EntB and EntF (Figure 4.1). These many levels of selection likely preclude all but a small proportion of the total PPTase genes in each metagenomic library from discovery - indeed Charlop-Powers et al. found only Sfp type PPTase enzymes were able to complement the enterobactin siderophore biosynthesis pathway ${ }^{101}$. As over-sampling in this screening process is desirable, and to balance interests in gaining high rates of coverage per cluster of interest, with the labour involved in recovering each colony, 1,500 cosmids were set as a target per library.

After the results shown in Table 4.1 were obtained, a more considered strategy was applied to avoid enrichment bias. From the $\mathrm{CH} \Delta e n t D$ screening process I had observed $70-150$ colonies per final round screening plate, and here aimed to recover 1,500 colonies per library. I therefore divided the 10.55 million cosmid HM library into 15 screening pools of $\sim 7 \times 10^{5}$ unique clones each, combining library row pools to achieve this. The same process was followed for the RX (8.69 million cosmids) and SL libraries (13.27 million cosmids), where screening pools of $\sim 5.8 \times 10^{5}$ and $\sim 8.9 \times 10^{5}$ clones were prepared respectively. In each instance, after two rounds of screening, 1,500 colonies were picked (i.e. a further 4,500 colonies), which were then cultured separately before being combined with the other cultures from their respective libraries, and miniprepped to extract the cosmids. The three resulting cosmid pools (HM $\Delta e n t D$ pool, RX $\Delta e n t D$ pool, SL $\Delta e n t D$ pool) were each separately sequenced using both PacBio RSII and Illumina HiSeq 2x100 bp PE chemistries.

\subsection{Assembly of DNA sequence data}

Optimisation of sequence assembly parameters was performed with the HM $\Delta e n t D$ pool sequence data. This data was used rather than the $\mathrm{CH}$ AentD data (which was acquired first), as it made more sense to use a large dataset for parameter optimisation (1500 colonies in the HM $\Delta e n t D$ dataset and only 263 in the $\mathrm{CH} \Delta e n t D$ dataset).

Each dataset consisted of a combination of PacBio long reads and Illumina HiSeq 2500100 bp PE short reads. The DNA sequence encoding biosynthetic gene clusters are often very repetitive (due to the repeating domain structure of the encoded megasynth(et)ase enzymes), and highly conserved (repeated domains have high sequence identity). We reasoned that the PacBio long read data would best be able to resolve the repetitive 
sequences of highly conserved regions, and that the relatively high error rate of PacBio sequencing technology could be overcome by the highly accurate short read Illumina sequencing data.

The SPAdes genome assembler program was used for NGS data assembly ${ }^{129}$. This program is widely used in the literature for NGS data assembly, and has "hybrid assembly" functions (hyrbidSPAdes), that can take in a combination of long and paired end short read data ${ }^{130}$. In addition, the metagenome assembly function (metaSPAdes) has been specially designed to assemble potentially fragmented and diverse metagenome data ${ }^{131}$.

Initial attempts to assemble the NGS data resulted in long contig sequences with the pWEB/pWEB::tnc vector backbone interspersed in the sequences. This caused issues with resolving the biosynthetic gene clusters contained in the sequences, as the biosynthetic gene cluster was often divided by insertion of the cosmid vector. These could not always be removed post assembly, as some assembled sequences did not contain the full metagenome insert for the cosmid, so could not necessarily be circularised. Those that were "complete", often also partially repeated the sequence, in a sense reading around the circular cosmid multiple times. Another common assembly error was the erroneous concatenation of separate cosmid sequences, linked by the vector sequence which all cosmids share. All of these issues resulted in a need to remove the vector sequence prior to NGS data assembly.

A custom shell script was used (decontam.sh written by $\mathrm{PhD}$ student Mathew Storey, Appendix A) that combined the removal of contaminating DNA sequences (in this case, pWEB/pWEB::tnc vector backbone, and E. coli genomic DNA) from raw Illumina paired end read data, in a pipeline along with read quality assessment, and trimming low quality base calls and sequencing adapters from the reads. Quality assessment was performed at the start with FastQC, followed by removal of contaminating sequences using bowtie ${ }^{132}$. Trimmomatic then removed adapter sequences and low quality base calls ${ }^{133}$, followed by another FastQC analysis to ensure the quality of the output reads. PacBio raw read files were concatenated into a single .fastq file for assembly.

Using these input files, six assembly parameters were evaluated, with the Illumina reads used either as unprocessed input, vector decontaminated prior to assembly, or vector decontaminated and quality trimmed prior to assembly. These were assembled either as Illumina only input (standard SPAdes), with Illumina and PacBio input (hybridSPAdes), and with or without the --meta parameter flagged (metaSPAdes). Assemblies were 
evaluated based on the length of the longest assembled contig, the number of contigs greater than $30 \mathrm{~kb}$ (cosmid inserts expected to be 30-40 kb), and the proportion of Sanger sequencing cosmid end reads that mapped correctly onto the ends of the assembled sequences.

The highest rated assembly in these metrics was a hybrid metaSPAdes assembly using both vector decontaminated and quality trimmed Illumina reads and PacBio long read input. These same assembly parameters were then applied to the remaining three datasets $(\mathrm{CH}$ $\Delta e n t D, \mathrm{RX} \Delta e n t D$, and SL $\Delta e n t D)$, which were also assessed in the same way for assembly quality.

By mapping the remaining ends of the vector sequences to the contigs, the number of "complete cosmid" sequences in each assembled dataset could be counted (i.e. how many contigs started with a vector sequence on one end, then contain $30-40 \mathrm{~kb}$ of intervening sequence before ending with vector sequence on the final end), summarised in Table 4.2. The longest 500 contigs in each assembled dataset were extracted, and analysed using antiSMASH v4 for biosynthetic gene cluster identification ${ }^{22}$. The number and type of identified gene clusters are summarised in Table 4.2. In each of the screened metagenome library sequence datasets, antiSMASH found multiple biosynthetic gene cluster sequences, with a bias toward NRPS, PKS and hybrid biosynthesis systems. This was the expected result, as the metagenome screening strategy selected for PPTase gene containing cosmids, and PPTase genes are required for activation of NRPS and PKS biosynthesis.

\begin{tabular}{llll|llll} 
& & & & \multicolumn{4}{c}{ Cluster type } \\
\hline Assembly & $\begin{array}{l}\text { Contigs } \\
\text { >30kb }\end{array}$ & $\begin{array}{l}\text { “complete } \\
\text { cosmids" }\end{array}$ & $\begin{array}{l}\text { antiSMASH } \\
\text { identified } \\
\text { clusters }\end{array}$ & $\begin{array}{l}\text { NRPS } \\
\text { clusters }\end{array}$ & $\begin{array}{l}\text { PKS } \\
\text { clusters }\end{array}$ & $\begin{array}{l}\text { NRPS/PKS } \\
\text { hybrid } \\
\text { clusters }\end{array}$ & Other \\
\hline CH $\Delta e n t D$ & 69 & 64 & 68 & 20 & 21 & 16 & 11 \\
HM $\Delta e n t D$ & 268 & 221 & 155 & 60 & 19 & 46 & 30 \\
RX $\Delta e n t D$ & 136 & 106 & 135 & 93 & 7 & 23 & 12 \\
SL $\Delta e n t D$ & 210 & 164 & 145 & 72 & 22 & 28 & 23 \\
\hline Total & 683 & 555 & 503 & 245 & 69 & 113 & 76 \\
\hline
\end{tabular}

Table 4.2 - Summary table of sequence data assemblies and biosynthetic gene cluster identification for screened cosmid libraries. 


\subsection{Gene level diversity in metagenome biosynthetic gene clusters}

This analysis pipeline resulted in the identification of 503 gene clusters; 68 in the Castle Hill Basin library, 155 in the Half Moon Bay library, 135 in the Roxburgh library, and 145 in the Saint Anne's Lagoon library. Many of these biosynthetic gene clusters are partial clusters - either as a result of the full pathway not being captured on a single cosmid sequence (insert size typically $30-40 \mathrm{~kb}$ ), or the full pathway not being assembled in the NGS assembly process. For this level of analysis, completeness of the biosynthetic gene cluster sequence is not strictly important, as here I have investigated the diversity of individual gene sequences identified from the putative biosynthetic gene clusters. Later analysis into gene cluster level similarity does not assume completeness of biosynthetic gene cluster sequence, and therefore similarity metrics can be calculated from partial biosynthetic gene cluster sequences.

To interrogate the amino acid sequences encoded by the partial biosynthetic gene cluster sequences, the cosmid assemblies were first analysed by antiSMASH ${ }^{22}$. A custom python script (gbk_to_faa.py, Appendix A) was then used to extract individual predicted gene product amino acid sequences for each biosynthetic gene cluster from the antiSMASH generated Genbank files. A single amino acid fasta file was generated in this way for each metagenomic library. In addition to the four metagenome libraries created in this study, the $\Delta e n t D$ selected cosmid sequencing data from Charlop-Powers et al. 2013 was included in this analysis, hereafter referred to as the Rockefeller University library (RU). The RU $\Delta e n t D$ data was generated by a similar screening technique used in this study, from a metagenomic library of American soil origin. The DNA sequence of $100 \Delta e n t D$ selected cosmids was made publicly available by Charlop-Power et al., and antiSMASH analysis detected 55 biosynthetic gene clusters in this dataset. A total of 11,124 ORFs/amino acid sequences were recovered from the 558 biosynthetic gene clusters using this method $(\mathrm{CH}$ $\Delta e n t D=1416, \mathrm{HM} \Delta e n t D=3403, \mathrm{RX} \Delta e n t D=2310, \mathrm{SL} \Delta e n t D=2741, \mathrm{RU} \Delta e n t D=1254$ sequences).

\subsubsection{Taxonomic diversity of metagenome biosynthetic gene cluster genes}

In most cases, the biosynthetic gene clusters enriched in our data did not contain $16 \mathrm{~S}$ or other robust phylogenetic markers, necessitating an alternative approach for elucidating 
putative phylogenetic origins. Each of the detected open reading frames were analysed using Diamond BLAST taxonomic classification, to generate the closest associated NCBI taxonomic ID (taxID) ${ }^{116}$. TaxonKit was used to generate complete tax ID reference lists for each major soil bacterium phyla ${ }^{134}$. Then, a custom pipeline written in python (allsoil_taxa.ipynb, Appendix A) was used to classify the query taxIDs at the phylum level according to the generated reference lists. Results present above phylum level classification were disregarded (i.e. ORF classification at the level of "bacteria", superphylum groupings, "uncultured organism", or "no result" were removed from analysis - 6,392 of 11,124 ORFs), as were ID classifications not grouped into one of the specified phyla (77 of the 11,124 ORFs).

Also included in this analysis pipeline were two database sequence sets, the first of which were the protein sequences in the curated "Minimum Information about a Biosynthetic Gene" (MIBiG) repository v1.4 ${ }^{21}$. From the MIBiG dataset, 1,999 protein sequences out of $31,023(6.4 \%)$ were excluded from the analysis for the same reasons as above. The MIBiG database represents the largest centralised and experimentally verified collection of biosynthetic gene clusters, and serves here to provide a snapshot of the current state of knowledge for bacterial secondary metabolism genes. From the 1830 biosynthetic gene clusters in the MIBiG database, less than 30 are tagged with "uncultured" organism origin. Therefore, this database can be used as a model for biosynthetic gene clusters found in traditional culture based natural product discovery pipelines. In all analyses in this chapter, comparisons to the MIBiG database will be made, to provide insight into the differences in genetic/biosynthetic diversity found in soil metagenomes relative to the current state of knowledge of biosynthetic gene clusters. The other database set included in this analysis was all $\sim 8,000$ bacterial genomes in the NCBI database. These genome sequences were processed in a manner intended to mirror the experimental PPTase screening applied to the soil metagenome libraries (section 4.4). The genomes were parsed through antiSMASH to identify biosynthetic gene cluster sequences, and from this set, only .gbk files containing both a PPTase (4446 clusters) and classified as NRPS and/or PKS biosynthetic gene clusters were used (2458 clusters). This dataset contained 115,751 protein sequences, and $22,983(19.9 \%)$ were excluded for not being assigned to one of the major phyla.

The resulting taxonomic distributions of ORFs in each library and database were plotted in a split bar chart for visualisation (Figure 4.2) and described in Table 4.3. This analysis pipeline is not intended to give a representation of the phylogenetic distribution of the 
bacteria species present in each soil environment, but rather a relative measure of each phylum's contribution to the aggregate secondary metabolism of the soil. The database sequence distributions, likewise are displayed for comparison purposes to show how the soil metagenome datasets compare to the current state of knowledge on bacterial secondary metabolism, whether that be in dedicated biosynthetic gene cluster database (MIBiG), or in the context of all known bacterial genome sequences (NCBI).
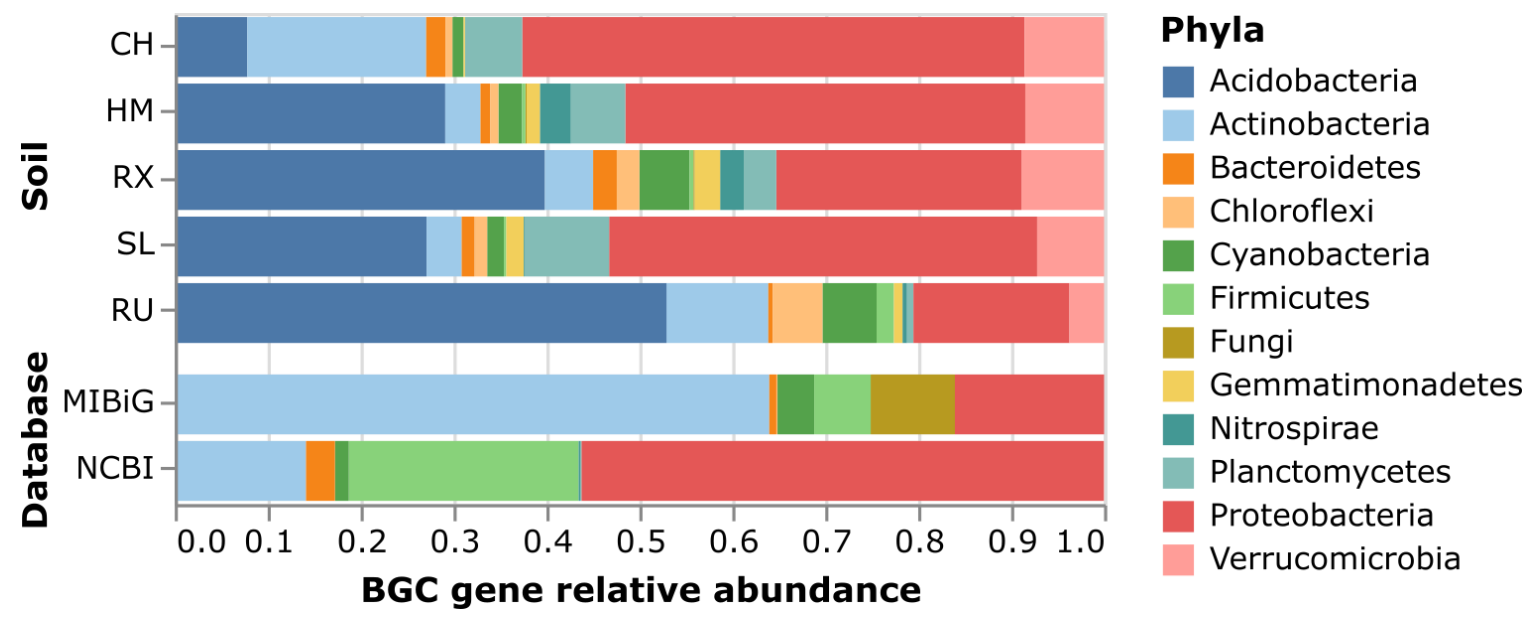

Figure 4.2 - Taxonomic distribution of open reading frames in metagenomic biosynthetic gene clusters. Open reading frames associated with biosynthetic gene clusters from the four soil metagenome libraries created in this study (CH, HM, RX, SL), and identically collected publicly available data from Rockefeller University (RU) were assigned to phyla. The open reading frames from two database sets of biosynthetic gene clusters: MIBiG, and all PPTase containing modular biosynthetic gene clusters from NCBI bacterial genomes, were classified in the same way. ORFs not assigned to the phylum level or below were discarded. Plots were created using Python, with the Vega based visualisation library Altair.

The five soil libraries have notably different distributions of bacterial phyla, and are also markedly different from the MIBiG and NCBI data in this regard (Figure 4.2). While Actinobacteria are often referred to as a biosynthetically gifted phylum (with many antibiotics having been discovered in Actinobacterial Streptomyces sp.) biosynthetic genes recovered in this study and classified as Actinobacterial were in the minority for each soil studied. The greater relative abundance of Actinobacteria in the database sets is likely a result of historical "inertia" in research efforts - two-thirds of microbial derived drugs came from streptomycetes, encouraging future bias towards these clades ${ }^{69,135}$. This figure maps almost perfectly onto the $64 \%$ of biosynthetic gene cluster genes sequences in MIBiG being classified as Actinobacterial in origin. From this analysis it would seem the field of 
microbial natural products discovery is biased towards actinomycetes in a way that the distribution of biosynthesis genes in the environment is not. Likewise, the NCBI dataset contains many actinomycete derived biosynthetic gene clusters, likely owing to DNA sequencing of actinomycetes for genome mining efforts. The other abundant phyla are likely overrepresented in the biosynthetic gene clusters present in NCBI as a result of these organisms being studied for other purposes (Bacteroidetes, Firmicutes and Proteobacteria are all abundant in many different environments, and contain common commensal and pathogenic species).

In each soil metagenome library, the majority of secondary metabolism genes classify as either Acidobacteria or Proteobacterial in origin, although the relative distribution of these groups varied widely between metagenomes. Other understudied bacterial phyla including the Chloroflexi, Gemmatimonadetes, Nitrospirae, Planctomycetes, and Verrucomicrobia, have significant contributions to soil secondary metabolism with either extremely low, or no representation in MIBiG (Table 4.3). The biosynthetic potential of these clades is likewise underrepresented in the NCBI sequence database, demonstrating these clades are understudied in a general sense - not solely for biosynthesis. It is apparent that the experimental workflow described in this chapter provide access to biosynthetic diversity that has been missed by culture-based discovery efforts.

\begin{tabular}{llll}
\hline Phyla & $\begin{array}{l}\text { Metagenome } \\
\text { ORFs }(\boldsymbol{\%})\end{array}$ & $\begin{array}{l}\text { MIBiG ORFs } \\
(\boldsymbol{\%})\end{array}$ & $\begin{array}{l}\text { NCBI ORFs } \\
(\boldsymbol{\%})\end{array}$ \\
\hline Acidobacteria & 29.9 & - & 0.2 \\
Actinobacteria & 6.9 & 64.0 & 13.9 \\
Bacteroidetes & 1.5 & 0.8 & 3.1 \\
Chloroflexi & 1.7 & 0.1 & - \\
Cyanobacteria & 3.1 & 4.0 & 1.5 \\
Firmicutes & 0.4 & 6.1 & 24.7 \\
Fungi & 0.1 & 9.1 & - \\
Gemmatimonadetes & 1.6 & - & - \\
Nitrospirae & 1.6 & - & 0.2 \\
Planctomycetes & 5.8 & - & 0.1 \\
Proteobacteria & 39.6 & 16.1 & 56.2 \\
Verrucomicrobia & 7.8 & - & 0.1 \\
\hline
\end{tabular}

$\overline{\text { Table } 4.3 \text { - Phylum distribution table of open reading frames from metagenome sequences, }}$ and MIBiG and NCBI databases. 


\subsubsection{Functional diversity of metagenome biosynthetic gene cluster genes}

By comparing the predicted amino acid sequences from identified biosynthetic gene clusters to known database sequence, inferences can be made both about the potential biological function of the predicted genes, and about the novelty encoded. One of the major reasons for investigating metagenome libraries for natural product biosynthesis, is to discover genes with low identity to known genes, and are therefore likely to provide novelty in the natural product that results from the metabolic pathway.

Different studies have used different thresholds for correlating sequence homology level to functional gene novelty ${ }^{16,89,101,136}$. Here I chose to use a low threshold value of 50\% protein level homology. It is likely that a far higher identity score could be justified for novelty as two genes with 50\% amino acid homology are likely only distantly related, and can have very different functions - however due to the speculative nature of this analysis I decided to be conservative.

Each of the five amino acid fasta files were compared to the curated MIBiG v1.4 database ${ }^{21}$, by using protein blast (BLASTp) analysis against a custom blast database generated using the MIBiG amino acid fasta data set. The results of this analysis are summarised in Table 4.4. As the MIBiG database is the largest repository for experimentally verified natural product biosynthetic gene clusters, and has over $98 \%$ of entries originating in cultured organisms, similarity to MIBiG sequence data will be used as a metric for novelty of gene function in small molecule biosynthesis found in the metagenome libraries.

The Roxburgh library was the only library to contain an amino acid sequence that was identical (100\% identity) to one from the MIBiG reference database. No other library contained a sequence with greater than $89 \%$ identity to a sequence in that database. For each library, the average percent identity to a database sequence was less than $40 \%$ (both mean and median percentage identity). 


\begin{tabular}{llllll}
\hline & $\begin{array}{l}\text { CH } \\
\text { library }\end{array}$ & $\begin{array}{l}\text { HM } \\
\text { library }\end{array}$ & $\begin{array}{l}\text { RX } \\
\text { library }\end{array}$ & $\begin{array}{l}\text { SL } \\
\text { library }\end{array}$ & $\begin{array}{l}\text { RU } \\
\text { library }\end{array}$ \\
\hline Number of amino acid sequences & 1404 & 3375 & 2293 & 2727 & 1242 \\
Maximum percentage identity & 85.5 & 88.9 & 100 & 82.7 & 84.3 \\
Mean percentage identity & 38.3 & 36.9 & 38.1 & 36.9 & 38.3 \\
Median percentage identity & 36.1 & 35.5 & 36.5 & 35.3 & 36.8 \\
Minimum percentage identity & 19.6 & 18.1 & 15.7 & 18.6 & 18.6 \\
Percentage of sequences with $\geq 50 \%$ identity & 12.5 & 9.8 & 12.6 & 9.6 & 11.8 \\
Percentage of sequences with $<50 \%$ identity & 87.5 & 90.2 & 87.4 & 90.4 & 88.2 \\
\hline
\end{tabular}

Table 4.4 - Metagenome library biosynthetic gene cluster amino acid sequence identities to nearest homologue MIBiG reference database. The amino acid sequences from each antiSMASH analysed pool of metagenome biosynthetic gene clusters were extracted and then compared using BLASTp to the MIBiG reference database amino acid sequences.

The benchmark for novelty was set at sequences with a percentage identity to the closest matching database sequence of less than 50\%. Using this metric, each metagenome library assessed here had over $85 \%$ of the predicted gene products passing the benchmark into novelty. The "least novel" library by this metric was the Roxburgh library, which was still overwhelmingly novel, in that only $12.6 \%$ of the predicted gene products had amino acid sequence identity to a sequence in the MIBiG database greater than $50 \%$. This assignment of 'least novelty' is consistent with the Roxburgh library also having the highest maximum identity sequence of $100 \%$, and the highest median identity score for any of the metagenome libraries. The "most novel" library by this metric was the Saint Anne's Lagoon library, where only $9.6 \%$ of the predicted gene products had $>50 \%$ amino acid sequence identity to a sequence in the MIBiG database. This is concordant with the Saint Anne's Lagoon library also having the lowest mean and median identity sequence scores, and the lowest maximum identity score of any library created in this study.

This BLASTp analysis was then repeated, with each antiSMASH processed $\Delta e n t D$ amino acid fasta dataset compared to a custom reference dataset consisting of all the other soil metagenome datasets (i.e. $\mathrm{CH} \Delta e n t D$ was compared to a set consisting of $\mathrm{HM}+\mathrm{RX}+\mathrm{SL}+\mathrm{RU}$ $\triangle e n t D$ pool ORFs). The results of both this analysis, and the previous BLASTp analysis against the MIBiG database are summarised in histograms in Figure 4.3. 
Castle Hill $\Delta e n t D$ pool

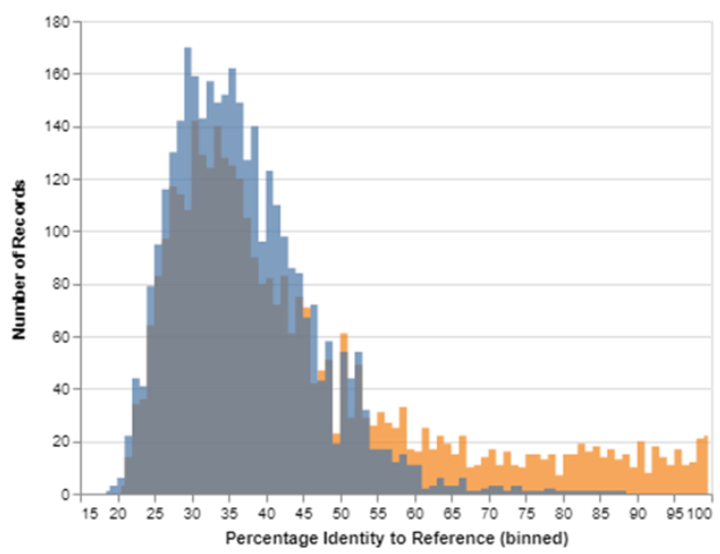

Roxburgh $\triangle e n t D$ pool

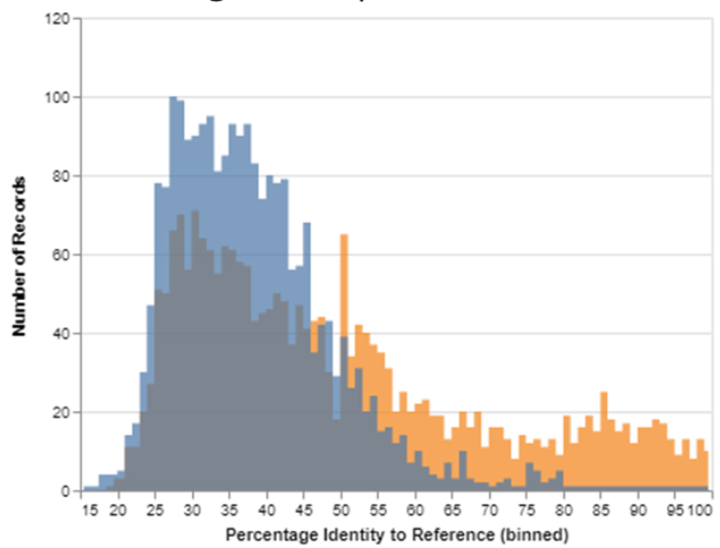

Rockefeller $\Delta e n t D$ pool

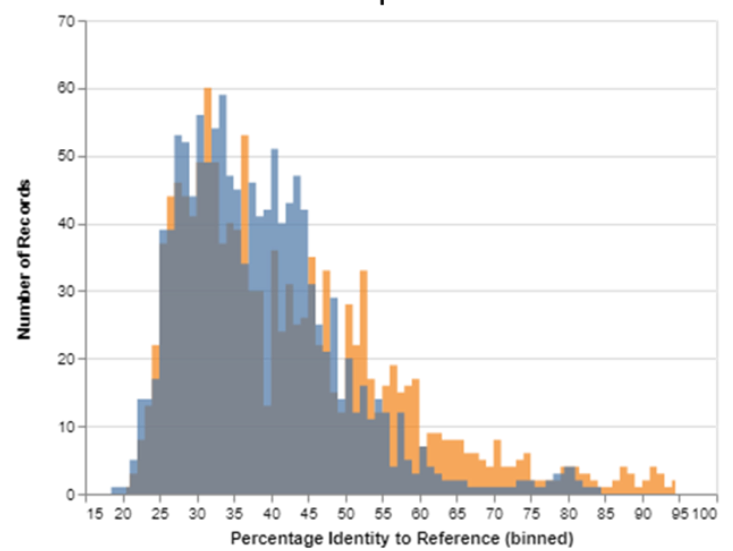

Half Moon Bay $\Delta e n t D$ pool

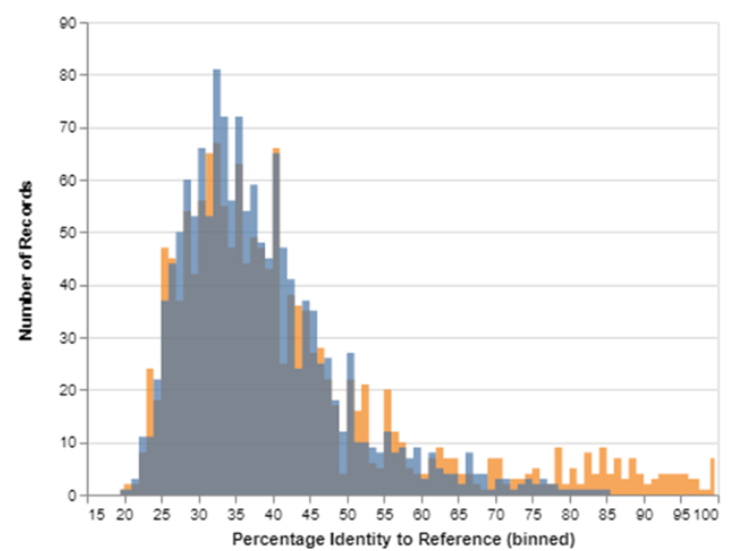

Saint Anne's Lagoon $\Delta e n t D$ pool

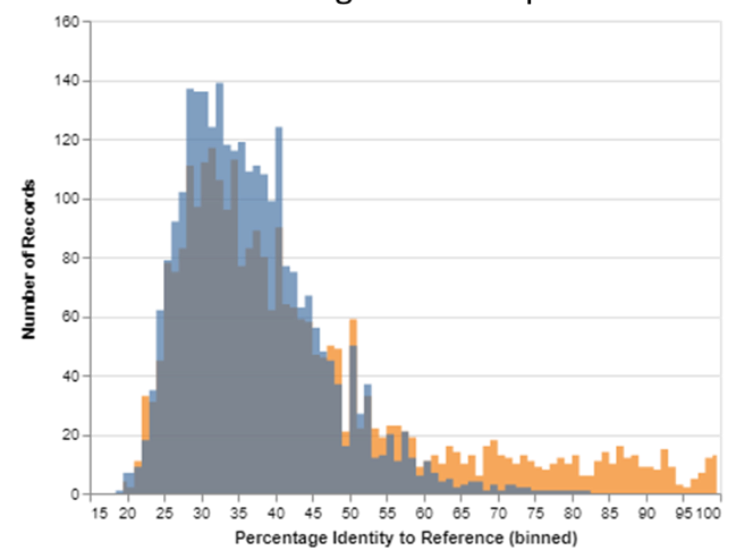

Metagenome compared to MIBiG database amino acid sequences

Metagenome compared to all other metagenome amino acid sequences

Figure 4.3 - Metagenome biosynthetic gene cluster amino acid identity plots. The amino acid sequences from each antiSMASH analysed pool of metagenome biosynthetic gene clusters were extracted and then compared to a reference database of amino acid sequences from either MIBiG (blue) or the remaining metagenome library pools (orange). Histograms were plotted according to the BLASTp highest percentage identity against frequency, with maximum binning set to 100. Plots were created using Python, with the Vega based visualisation library Altair. 
The data summarised in Table 4.4 can be more clearly interpreted in Figure 4.3. The histograms where each soil metagenome amino acid sequence was aligned to the sequences in the MIBiG database (in blue) illustrate that, in all cases, the soil metagenomes have low overall homology to the MIBiG database sequences (most sequences <50\% homology). This is interesting, as the MIBiG reference dataset is a verified collection of natural product biosynthetic gene clusters (mostly NRPS and PKS), and was therefore expected to contain a similar set of functionalities to the metagenome $\Delta e n t D$ biosynthetic gene cluster sequences.

Figure 4.3 also shows the results of the BLASTp analysis comparing each soil biosynthetic gene cluster amino acid sequence set to all other metagenome datasets (in orange). This analysis demonstrates that each soil has a higher degree of amino acid sequence similarity to other soil metagenomes than to sequences in published cultured databases (MIBiG). This relationship is even apparent for the RU library, which appears more related to New Zealand soil metagenomes than cultured sequences, many of which would be from American soils. Also important to note is that each soil, despite having sequences closely related to other soil metagenomes, also contains a majority of sequences that are unique (i.e. below $50 \%$ identity to anything in the metagenome dataset).

This analysis suggests that while soils metagenomes have some "shared identity", represented by the right-shifted orange histograms in Figure 4.3, the majority of sequences appear to be highly novel. Critically, while these highly novel sequences are accessible through metagenomic analysis, they do not appear accessible through standard isolation of lab-cultivable bacteria.

\subsection{Gene cluster level analysis}

Novelty of gene sequence, or the amino acid sequences encoded, as assessed in section 4.6 can provide indication of novel metabolism. However, it is the interaction of all of the genes in a biosynthetic pathway that contribute to the novelty of the final natural product output. To examine the biosynthetic gene cluster sequences as collections of genes, each of the $\Delta e n t D$ sequencing pool antiSMASH output datasets was combined along with the biosynthetic gene clusters from Charlop-Powers et al. 2013 (RU $\Delta e n t D$ ), and the full MIBiG database. This combined dataset was then analysed using the BiG-SCAPE and CORASON packages (Figure 4.4) ${ }^{117,118}$. BiG-SCAPE (Biosynthetic Gene Similarity 
Clustering and Prospecting Engine) takes biosynthetic gene cluster inputs and creates sequence similarity networks of the biosynthetic gene clusters, which are grouped into gene cluster families (GCFs). The networks are made by comparing each pairwise combination of biosynthetic gene clusters in the query set to generate a distance score. This score is calculated using a weighted combination of three metrics - the Jaccard index (JI) to measure the similarity of gene content, adjacency index (AI) to measure shared pairs of adjacent domains, and domain sequence similarity (DSS) index to measure the sequence differences between the query domains and a reference set. The combined score falls between 0 (identical clusters) and 1 (no shared features), and is weighted differently for biosynthetic gene cluster classes (e.g. type I PKS have higher weighting for DSS and JI score than other PKS systems, where AI is highly weighted) ${ }^{117}$. The pairwise distances, in addition with the user-defined cut-off values, are used to determine the families, and the network relationships. CORASON (CORe Analysis of Syntenic Orthologs to prioritize Natural Product-Biosynthetic Gene Clusters) constructs "multi-locus phylogenies" from groups of biosynthetic gene clusters (in this case, GCFs). This gives a more granular view of the relationships between similar biosynthetic gene clusters.

BiG-SCAPE analysis was trialled with a range of input parameter settings. Below a distance cut-off value of 0.5 , metagenome biosynthetic gene clusters would not link in networks with the MIBiG reference biosynthetic gene clusters. A cut-off value for similarity of 0.6 was used, as above this value, biosynthetic gene clusters with very few common features would form tight networks. However, even at high cut-off values of 0.9 or 1.0, it was rare for MIBiG dataset biosynthetic gene clusters to belong to the same gene cluster family as metagenome biosynthetic gene clusters - although links between families of MIBiG biosynthetic gene clusters and metagenome biosynthetic gene clusters were observed more often. The "Mixed" network parameter was used instead of the biosynthetic class-based networks (e.g. NRPS, PKSI etc.), as the mixed network gave a better overall analysis of hybrid biosynthesis (e.g. NRPS/PKS hybrids, heavily glycosylated NRPS biosynthetic gene clusters, etc.). Singletons were included in the network analysis, to provide a full overview of the data. The "glocal" alignment mode was used for the network construction, as this mode compares subsets of each biosynthetic gene cluster's pfam concatemers ("subclusters"), meaning that the algorithm can reliably align even partial or incomplete biosynthetic gene clusters to complete biosynthetic gene clusters without penalty. This is a key requirement for the data generated in this study, as the library 
construction and screening methods used to generate these sequencing pools capture a mixture of complete and incomplete biosynthetic gene clusters.

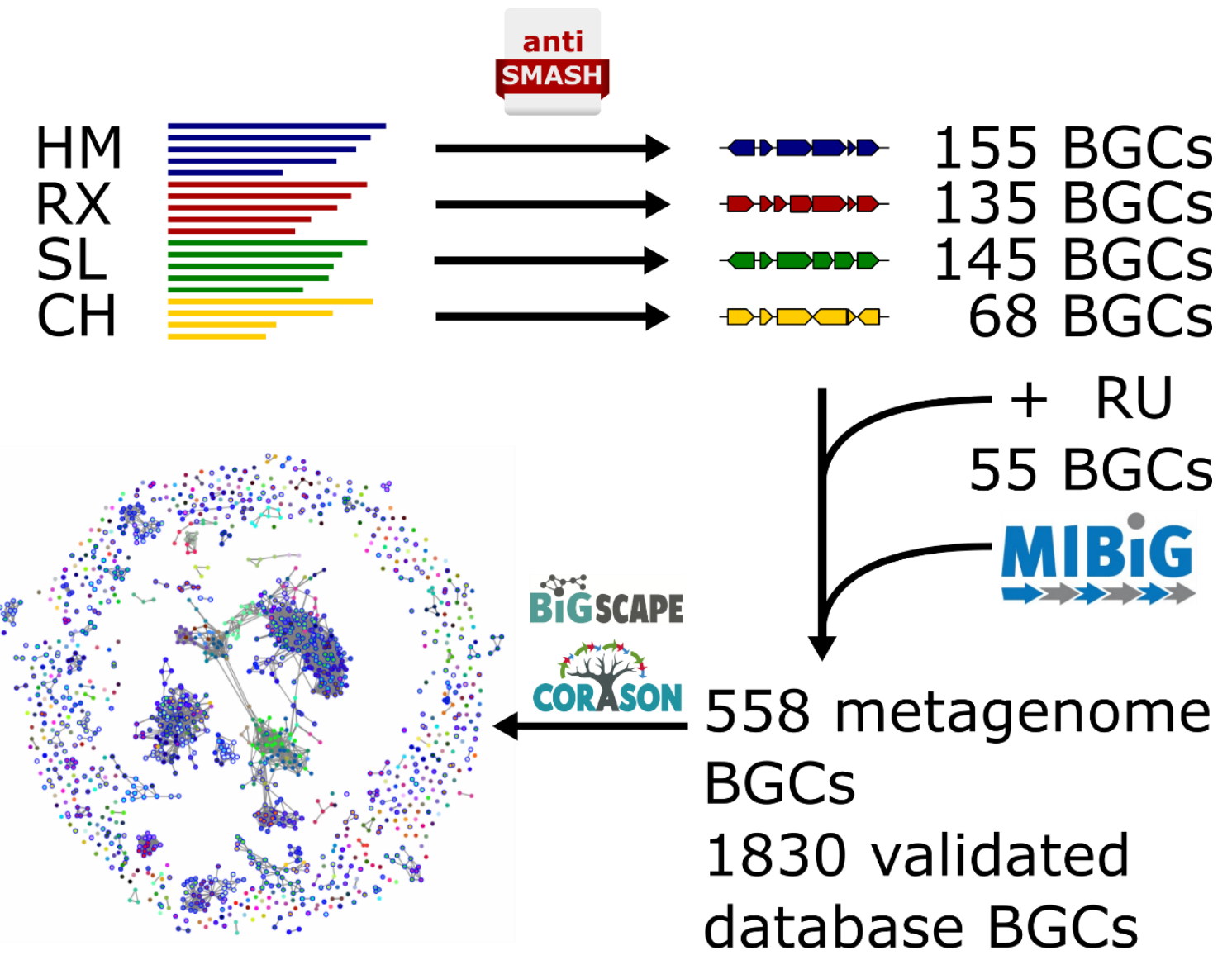

Figure 4.4 - Biosynthetic gene cluster analysis pipeline. DNA assemblies from the four screened metagenome libraries (and RU $\Delta e n t D$ ) were first analysed by antiSMASH v4 software, to identify biosynthetic gene clusters and predict gene function. The five metagenome datasets along with the MIBiG database biosynthetic gene clusters were then analysed using BiG-SCAPE to create networks of related biosynthetic gene clusters, grouping biosynthetic gene clusters into gene cluster families (GCFs). GCFs were also analysed using CORASON to create GCF phylogenetic trees. The BiG-SCAPE network displayed was created with the 558 metagenome biosynthetic gene clusters detailed in the figure, along with all MIBiG biosynthetic gene clusters, clustered at cut-off value 0.6 , and is a mixed network of all biosynthesis classes.

The network files generated by BiG-SCAPE were then transferred to Cytoscape for further analysis (Figure 4.5). In this network, nodes represent individual biosynthetic gene clusters (MIBiG clusters in blue, metagenome in red), linked relative to BiG-SCAPE gene cluster alignment scores. Large network hubs of gene cluster families linked to type I PKS, NRPS, type II PKS/Saccharide, and TransAT-PKS secondary metabolism were identified, although each grouping did contain significant diversity (i.e. the NRPS network contained 
many hybrid biosynthetic gene clusters). Each of these large networks contained gene clusters from both the metagenome biosynthetic gene clusters found in this study and from the MIBiG database. Five smaller networks were also found to contain only metagenome biosynthetic gene clusters, and all were hybrid NRPS/PKS systems with very low identity to previously observed secondary metabolism. Most other metagenome biosynthetic gene clusters formed small two or three member networks, or singlets. Singlets were mostly very small biosynthetic gene clusters from small contigs out of the DNA sequence data assembly. 


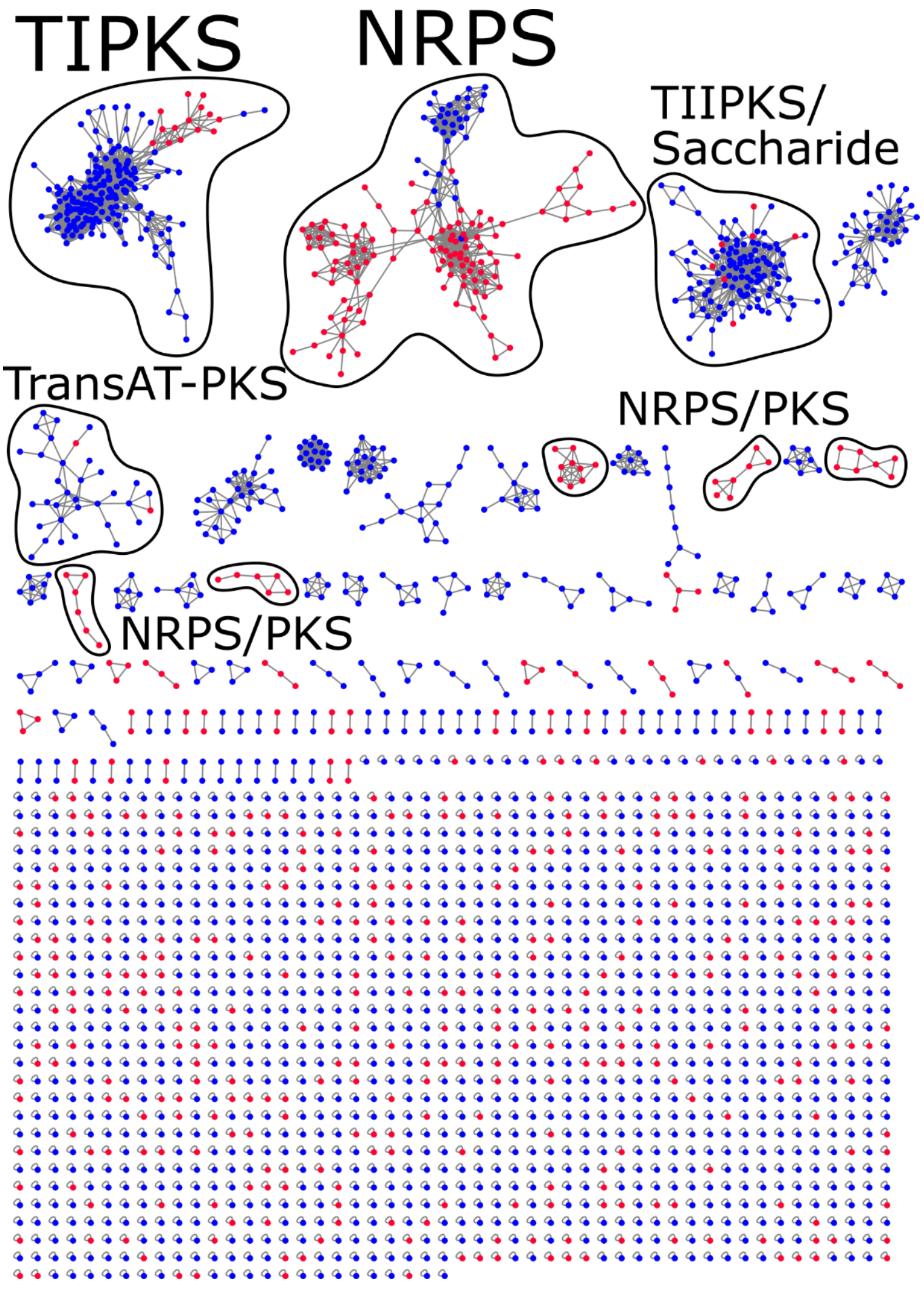

Figure 4.5 - BiG-SCAPE network diagram of all metagenome and MIBiG biosynthetic gene clusters. Nodes indicate either metagenome biosynthetic gene clusters (red) or MIBiG biosynthetic gene clusters (blue), with edges (grey) indicative of gene cluster similarity. The four major sub-networks containing at least one metagenome biosynthetic gene cluster are annotated with the major biosynthetic class represented. The next five smaller subnetworks containing solely 
metagenome biosynthetic gene clusters are all annotated NRPS/PKS, as these biosynthetic gene clusters all contain mixed biosynthesis systems, and have low similarity to any characterised biosynthetic gene cluster.

Three of the gene cluster families (GCFs) assigned by BiG-SCAPE were chosen to be investigated in more detail. GCF_01965 (Figure 4.7) and GCF_01987 (Figure 4.12) are a part of the large NRPS subnetwork hub that contains few MIBiG biosynthetic gene clusters, and therefore could represent some novel genetic and biosynthetic diversity. GCF_01980 (Figure 4.10) was selected as the BIG-SCAPE network showed the family as an outgroup of a subnetwork of type I PKS biosynthetic gene clusters from the MIBiG database (Figure 4.5 - TIPKS subnetwork). Each of these gene cluster families contain biosynthetic gene clusters from across multiple soil metagenomes, as detailed in Table 4.5, and contains unique biosynthetic features.

\begin{tabular}{llllllll}
\hline & CH & HM & RX & SL & RU & MIBiG & $\begin{array}{l}\text { Biosynthesis } \\
\text { class }\end{array}$ \\
\hline GCF_01965 & 0 & 2 & 6 & 3 & 0 & 0 & NRPS \\
GCF_01980 & 4 & 1 & 1 & 8 & 0 & 1 & TIPKS \\
GCF_01987 & 0 & 4 & 5 & 1 & 0 & 0 & NRPS \\
\hline
\end{tabular}

Table 4.5 - Details of gene cluster families. Gene cluster families (GCFs) were classified by BiG-SCAPE, and biosynthetic gene clusters were further analysed using the BiG-SCAPE pfam assignment feature, antiSMASH classifcations and BLASTp analysis. TIPKS = type I PKS.

Each of the metagenome derived biosynthetic gene clusters in the selected families (excluding the MIBiG entry in GCF_01980) were grouped and analysed by the same taxonomic classification pipeline used in 4.6.1. The results are provided in Figure 4.6. In each gene cluster family, one phylum is linked to the vast majority of gene function: Acidobacteria for GCF_01965 and GCF_01987, and Proteobacteria for GCF_01980. The three families analysed here were selected for their conserved relationship across soil metagenomes, and their divergence in the BiG-SCAPE network from MIBiG biosynthetic gene clusters. The biosynthesis patterns here appear to be "hidden" from culture-based discovery pipelines, and yet are abundant in this metagenomic analysis. It is therefore reasonable that these gene cluster families arise from phyla that are underrepresented in the 
MIBiG database relative to their representation in aggregate soil secondary metabolism (Section 4.6.1).

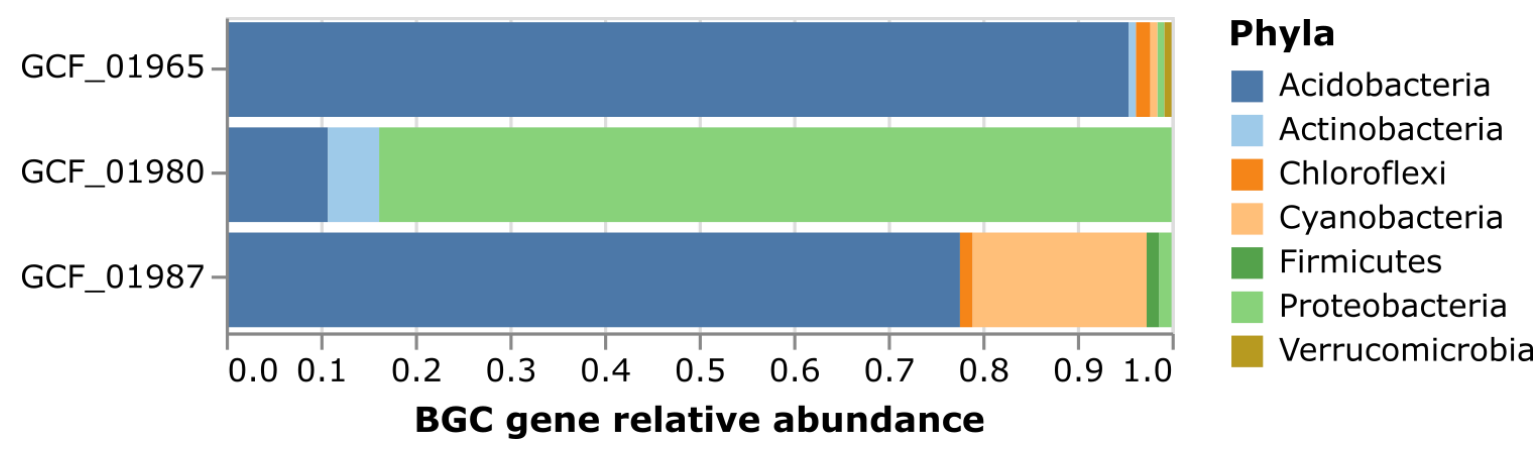

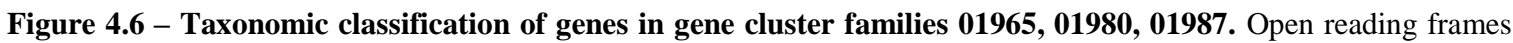
associated with GCF_01965, GCF_01980, GCF_01987 biosynthetic gene clusters were assigned to phyla. This was performed using Diamond BLAST taxonomy classification and the NCBI taxonomy database. ORFs not assigned to the phylum level or below were discarded. Plots were created using Python, with the Vega based visualisation library Altair.

In addition to the use of BiG-SCAPE and CORASON, the clusterBLAST module output of antiSMASH is used in the following sections to discuss the relatedness of the biosynthetic gene clusters in each family to other known biosynthetic gene clusters. The clusterBLAST module performs an alignment search of a biosynthetic gene cluster against a reference set to find gene clusters with related gene function. This is a complementary approach to the BiG-SCAPE alignments (based on pfam annotation), and the results of both approaches are discussed in sections 4.7.1, 4.7.2, and 4.7.3.

All three gene cluster families examined here contain sequence level novelty in their core biosynthetic ORFs (i.e. low-level homology A-domain/KS-domain), and extensive tailoring functionalities. It is often not possible to predict with accuracy the exact function of specific tailoring enzymes (e.g., a glycosyl-transferase enzyme may attach a sugar to a core natural product scaffold, but which specific sugar and where it attaches must be determined experimentally). However, the presence of multiple tailoring functions indicates evolutionary selection for a highly modified natural product, which can correlate to more biological specificity or potency ${ }^{17,32,137}$. 


\subsubsection{Gene cluster family 01965}

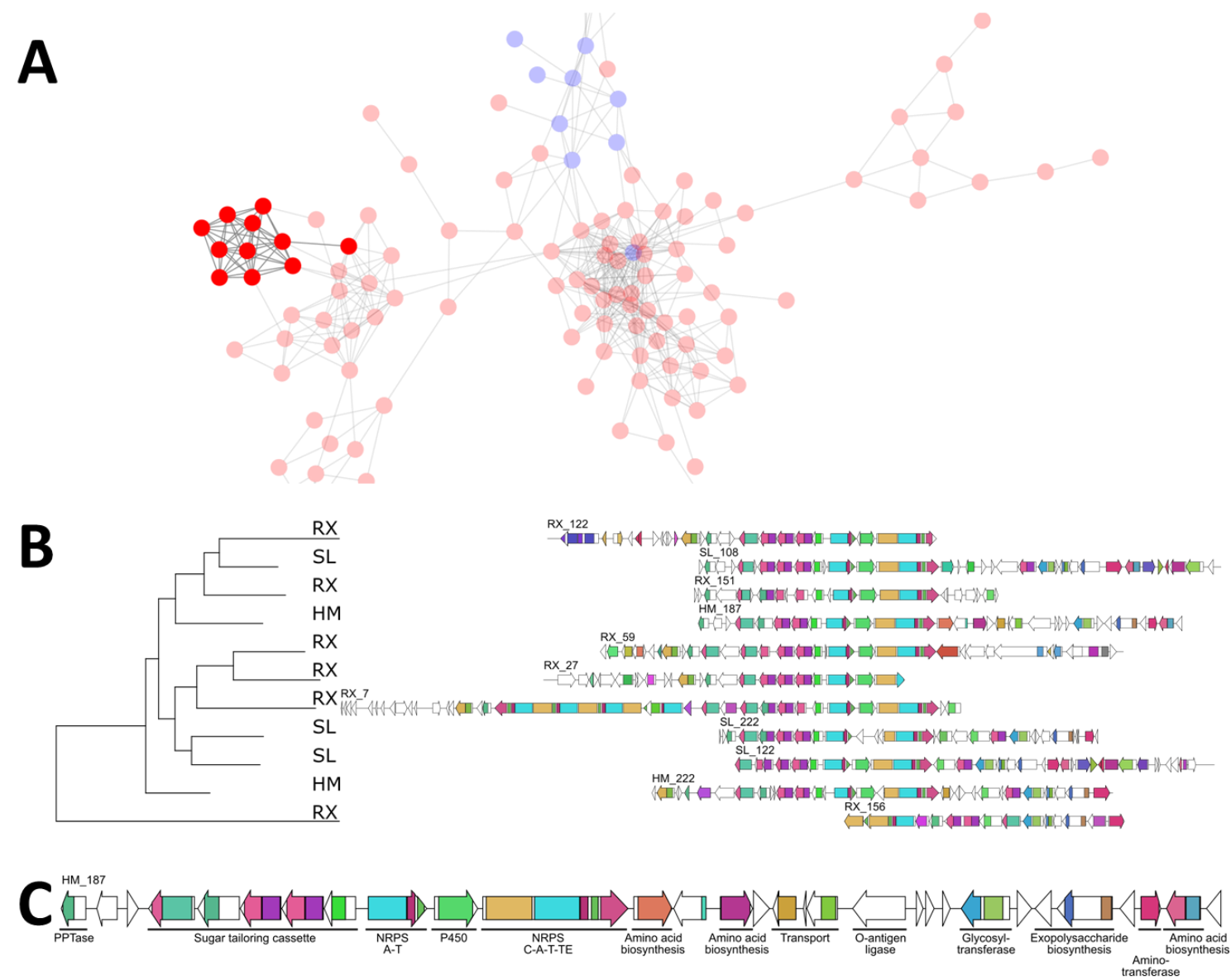

Figure 4.7 - Gene cluster family 01965 (GCF_01965). A) BiG-SCAPE network extracted into Cytoscape, where nodes represent biosynthetic gene clusters from soil metagenomes (red) and MIBiG database biosynthetic gene clusters (blue). The eleven nodes belonging to GCF_01965 are highlighted. B) CORASON phylogenetic tree of the biosynthetic gene clusters in GCF_01965. The left graph plots the phylogenetic tree annotated with soil origin of each biosynthetic gene cluster. The gene cluster ORFs are depicted on the right, centred around the conserved ORFs that define the gene cluster family. C) HM_187 biosynthetic gene cluster is shown in detail, with major natural product biosynthesis genes annotated.

GCF_01965 is a family of small NRPS gene clusters, mostly containing one A-T and one C-A-T-TE module (with the exceptions of RX_7, which contains all of the core family modules, in addition to a secondary larger NRPS system, and RX_156 which appears to be a partial gene cluster). The A-domain of the A-T module is predicted to select for leucine in all cases, and the prediction for the substrate specificity of the C-A-T-TE module is uncertain (Figure 4.8); pHMM predicts either glycine or valine, SANDPUMA predicts alanine, SVM predicts a "hydrophilic amino acid", and the Stachelhaus prediction is a weak 
match for glutamic or aspartic acid. These predictions do not reach a reasonable consensus, as glycine, valine and alanine are aliphatic, and glutamic/aspartic acid are charged with far larger side chains. It is possible that the A-domain selects a modified, non-proteogenic amino acid, as multiple amino acid tailoring/biosynthesis enzymes are present across the gene cluster family. Alternatively these A-domains may represent alternative binding modes, or monomers that are not captured in the training data that underpins existing prediction algorithms (e.g. only one methionine selective A-domain has ever been characterised $\left.^{138-140}\right)$.
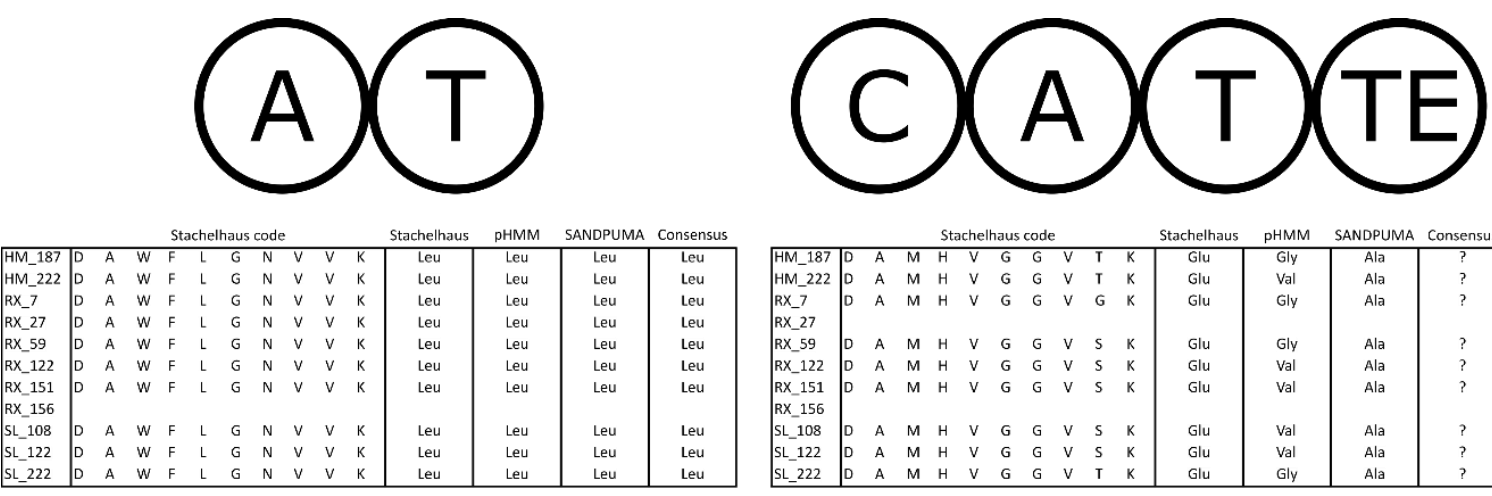

\begin{tabular}{|c|c|c|c|c|c|c|c|c|c|c|c|c|c|c|}
\hline & & & & & chelha & ausc & & & & & Stachelhaus & PHMM & SANDPUMA & Consensus \\
\hline HM_187 & D & A & M & $\mathrm{H}$ & $\mathrm{V}$ & & 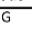 & $\mathrm{v}$ & $T$ & K & Glu & Gly & Ala & $?$ \\
\hline HM_222 & D & A & M & H & v & G & G & v & T & K & Glu & Val & Ala & \\
\hline $\begin{array}{l}R X_{1}=7 \\
R X_{2} 27\end{array}$ & D & A & M & $\mathrm{H}$ & $v$ & G & G & $v$ & G & k & Glu & Gly & Ala & ? \\
\hline RX_59 & D & A & M & H & $\mathrm{v}$ & G & G & v & s & k & Glu & Gly & Ala & ? \\
\hline RX_122 & D & A & M & $\mathrm{H}$ & $\mathrm{v}$ & G & G & v & $\mathrm{s}$ & k & Glu & Val & Ala & ? \\
\hline RX_151 & D & A & M & H & v & G & G & $\mathrm{v}$ & s & k & Glu & Val & Ala & ? \\
\hline RX_156 & & & & & & & & & & & & & & \\
\hline SL_108 & D & A & M & H & v & G & G & v & s & $\mathrm{k}$ & Glu & Val & Ala & ? \\
\hline SL_122 & D & A & M & н & $\nabla$ & G & G & $\nabla$ & s & k & Glu & Val & Ala & ? \\
\hline SL- 2222 & $D_{D}$ & A & M & $\mathrm{H}$ & $\mathrm{v}$ & G & G & $\mathrm{v}$ & I & K & Glu & Gly & Ala & $?$ \\
\hline
\end{tabular}<smiles>CC(C)CC(N)C(=O)O</smiles><smiles>[R]C(N)C(=O)O</smiles><smiles>[R]C(NC(=O)C(N)CC(C)C)C(=O)O</smiles>

Figure 4.8 - Adenylation domain monomer selectivity for each biosynthetic gene cluster in GCF_01965. No consensus monomer could be predicted for any of the A-domains in the C-A-T-TE module, while the A-T module always selected leucine. The unknown monomer is depicted as an amino acid with an " $\mathrm{R}$ " sidechain.

Additional amino acid biosynthesis or tailoring functionality is present in all clusters except for RX_151. Five gene clusters have a methionine aminopeptidase two genes downstream from the PPTase gene. Others include aminotransferase enzyme acting as a cysteine 
desulfurase (6/11), cysteine synthase (3/11), ornithine cyclodeaminase (1/11), and homoserine dehydrogenases (3/11) genes. All biosynthetic gene clusters encoding a cysteine synthase also encode cysteine desulfurase, suggesting a metabolic pathway $\left(\mathrm{O}_{3}-\right.$ acetyl-L-serine $\rightarrow$ L-cysteine $\rightarrow$ L-alanine) that may be involved in the overall gene cluster output. Another point of note, 8/11 biosynthetic gene clusters have either methionine aminopeptidase or homoserine dehydrogenase genes, which liberate methionine, or produce intermediates of methionine biosynthesis respectively.

\begin{tabular}{lccccc}
\hline & $\begin{array}{l}\text { Methionine } \\
\text { aminopeptidase }\end{array}$ & $\begin{array}{l}\text { Cysteine } \\
\text { desulferase }\end{array}$ & $\begin{array}{l}\text { Cysteine } \\
\text { synthase }\end{array}$ & $\begin{array}{l}\text { Ornithine } \\
\text { cyclodeaminase }\end{array}$ & $\begin{array}{l}\text { Homoserine } \\
\text { dehydrogenase }\end{array}$ \\
\hline HM_187 & 1 & 1 & 1 & 1 & 1 \\
HM_222 & 1 & 1 & & & \\
RX_7 & 1 & & & & \\
RX_27 & 1 & & & & \\
RX_59 & 1 & & & & \\
RX_122 & 1 & & & & \\
RX_151 & & 1 & & \\
RX_156 & & 1 & 1 & & 1 \\
SL_108 & & 1 & 1 & & \\
SL_122 & & 1 & & & \\
SL_222 & & 1 & & \\
\hline
\end{tabular}

Table 4.6 - Amino acid biosynthesis genes present in GCF_01965.

The gene arrangement of the biosynthetic gene cluster is highly conserved across the gene cluster family, and there are a large number of conserved tailoring enzymes present. Nine out of eleven biosynthetic gene clusters in this family have a cytochrome P450 (P450) gene in between the two NRPS ORFs. Conserved domain search ${ }^{141}$ of the P450s central to almost all of the gene clusters in GCF_01965 returns P450s of diverse function. Of the three highest rated functions however, two are linked to different functions in to cyclodipeptide biosynthesis (either involved in cyclodipeptide synthesis or in cyclodipeptide side chain hydroxylation ${ }^{142}$ ), and the third is implicated in aiding glycosyltransferase function ${ }^{143}$. P450s are common tailoring enzymes in cyclodipeptide biosynthesis, which often involve NRPS enzymes at the core of the pathway ${ }^{144}$. The central location of the P450 gene in the NRPS cassette suggests a function in the core peptide biosynthesis, which may be the production of a cyclodipeptide ${ }^{145}$. 
Immediately upstream of the NRPS modules is a sugar tailoring cassette, consisting of a polysaccharide deacetylase, three or four glycosyl transferases, and a polysaccharide biosynthesis enzyme gene. Many of the gene clusters in this family have additional glycosyltransferases and polysaccharide biosynthesis genes throughout their clusters. Genes predicted to encode an O-antigen ligase/Wzy_C are present in six of the gene clusters, which may indicate additional polysaccharide biosynthesis. The functions of these gene products are generally difficult to predict, and may be implicated in the production of glycopeptide (glyco-cyclodipeptide), or perhaps the cluster linked polysaccharide biosynthesis may have a self-resistance function to the small molecule. No glycosylated cyclodipeptide has yet been described, however a previous genome mining study for cyclodipeptide biosynthesis did find associated glycosyltransferase genes ${ }^{146}$.
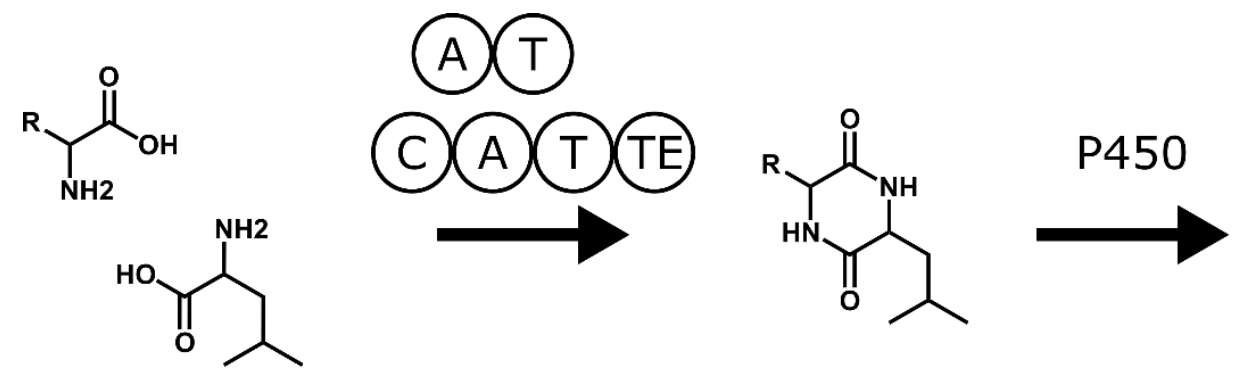<smiles>[R]C1NC(=O)C(C(O)C(C)C)NC1=O</smiles>
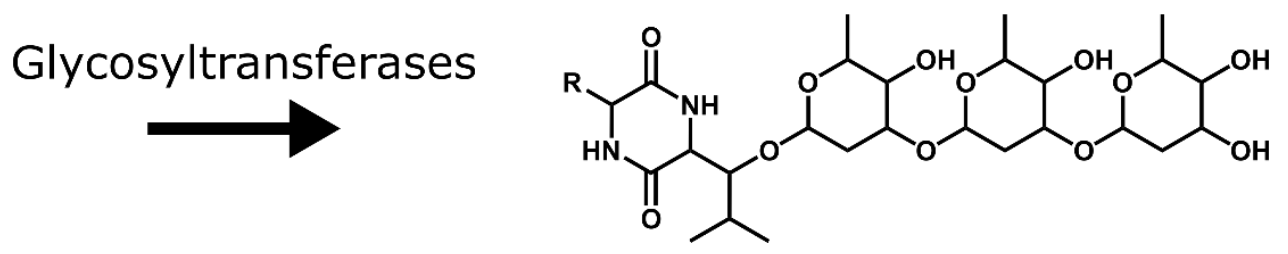

Figure 4.9 - Speculative biosynthesis scheme for generic GCF_01965 biosynthesis. In this proposed biosynthesis scheme, the leucine activated by the A-T module and the amino acid activated by the C-A-T-TE module are cyclised, likely by the TE-domain function. The P450 may act to hydroxylate an amino acid side chain (here depicted as $\beta$ hydroxylation of the lysine side chain, as the other amino acid in the cyclodipeptide is unknown). The hydroxylated side chain is then used as a substrate for three glycosyltransferase enzymes (here transferring deoxy-hexose sugars). The glycosyl-transferases may have multiple sites of sugar attachment, and each cluster in this family contains between three and six glycosyl-transferase genes.

A proposed biosynthesis scheme for a glyco-cyclodipeptide small molecule is given in Figure 4.9. This scheme suggests cyclodipeptide formation from leucine and an unspecified amino acid, where the leucine is $\beta$-hydroxylated, and then tri-glycosylated (each 
biosynthetic gene cluster contains between three and six glycosyltransferase genes). This biosynthesis scheme is speculative, although bears some resemblance to other cyclodipeptides ${ }^{145}$, including the cyclomarazines ${ }^{147}$ and thaxtomin ${ }^{142,148}$. It is also possible the biosynthetic gene clusters of this family produce a glycosylated linear-dipeptide, or a different arrangement of the two amino acids activated by the NRPS systems.

An antiSMASH clusterBLAST analysis suggested low overall similarity to any known NRPS biosynthetic gene cluster, and ten of the eleven biosynthetic gene clusters returned no result in a clusterBLAST search to any biosynthetic gene cluster (with more than one gene). RX_7 returned a 37\% identity to the nostopeptolide biosynthetic gene cluster, however this stemmed from the secondary NRPS ORF rather than the core family genes. This low similarity to any known biosynthetic gene cluster is particularly interesting, as members of GCF_01965 were found multiple times in all three of the large metagenome soil libraries screened in this study (HM, RX, SL), suggesting that while gene clusters of this type have not been previously observed, this biosynthesis scheme is relatively common in uncultivated soil bacteria. 


\subsubsection{Gene cluster family 01980}

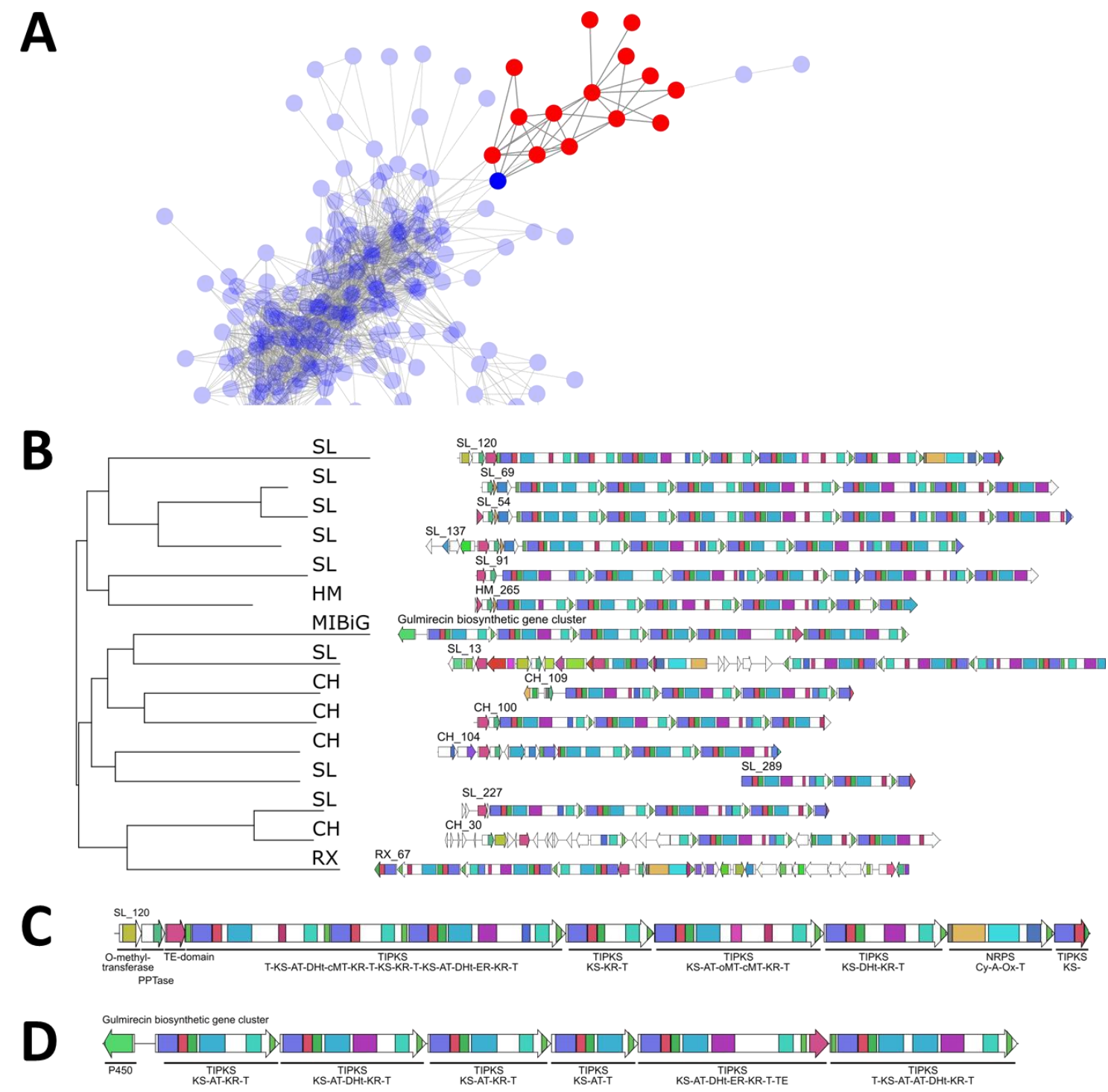

Figure 4.10 - Gene cluster family 01980 (GCF_01980). A) BiG-SCAPE network extracted into Cytoscape, where nodes represent biosynthetic gene clusters from soil metagenomes (red) and MIBiG database biosynthetic gene clusters (blue). The fifteen nodes belonging to GCF_01980 are highlighted. B) CORASON phylogenetic tree of the biosynthetic gene clusters in GCF_01980. The left graph plots the phylogenetic tree annotated with soil/database origin of each biosynthetic gene cluster. Biosynthetic gene cluster ORFs are depicted on the right. C) SL_120 biosynthetic gene cluster is shown in detail, with major natural product biosynthesis genes annotated. D) Gulmirecin is the lone MIBiG database biosynthetic gene cluster in this gene cluster family, and is annotated for reference.

GCF_01980 is a family of 14 multi-module type I PKS biosynthesis gene clusters, including a single entry from the MIBiG database - gulmirecin. Gulmirecin A and B are 
macrolide antibiotics (Figure 4.11), produced by a type I PKS gene cluster, and exhibit micromolar antibiotic activity against staphylococci without human cell cytotoxicity ${ }^{149}$. Like gulmirecin, all of the soil metagenome derived biosynthetic gene clusters in this family appear to encode highly reduced polyketides, with many DH-domains and 1-4 ERdomains per clusters. Most have at least one cis C-methyl transferase (cMT-) domain, and one cluster (SL_120) has two O-methyl transferase (oMT-) domains (one cis, and one trans). All of the metagenome biosynthetic gene clusters in GCF_01980 contain truncated genes on at least one edge of their sequence, suggesting these biosynthetic gene clusters are larger than the $\sim 35 \mathrm{~kb}$ that can be captured on a single cosmid, and that there are other biosynthetic features to these clusters not present in this dataset.

Three of the 14 metagenome biosynthetic gene clusters in this family have one complete NRPS module, which is not present in gulmirecin. One other $\left(\mathrm{CH}_{-} 109\right)$ contains a truncated C-domain, potentially indicating the start of an NRPS module on the complete biosynthetic gene cluster. The A-domain of the C-A-T module in RX_67 is predicted to select for glycine. The two Cy-A-Ox-T modules both have A-domains predicted to select for cysteine (in SL_13 and SL_120), an organisation known for cyclisation of cysteine to thiazole, as is seen in epothilone (Figure 4.11) biosynthesis ${ }^{41}$. Four of the metagenome biosynthetic gene clusters that do not contain any NRPS biosynthetic genes do contain an $m b t H$ gene, which function to aid in NRPS A-domain substrate selection, and have been used a "molecular beacon" to indicate NRPS biosynthesis in genome mining approaches ${ }^{36,150}$. This potentially indicates that these clusters, if complete, would indeed contain NRPS biosynthesis (HM_265, SL_54, SL_69, SL_137). These clusters bring the proportion of metagenome biosynthetic gene clusters in GCF_01980 to over half (8/14) with evidence of NRPS metabolism (complete/partial NRPS modules or $m b t H$ genes). The $m b t H$ genes all occur in a cassette consisting of a free TE, PPTase, $m b t H$ and a sulfotransferase. A sulfotransferase enzyme is also present immediately upstream of the truncated C-domain in $\mathrm{CH}_{-}$109.

Despite BiG-SCAPE grouping gulmirecin into GCF_1980, antiSMASH clusterBLAST analysis of all the metagenome biosynthetic gene clusters in this family indicated more similarity to oxazole/thiazole containing NRPS-type I PKS gene clusters (ajudazol ${ }^{53}$, epothilone $^{41}$, cystothiazole ${ }^{52}$ - Figure 4.11), although with low overall similarity scores between $16 \%-53 \%$. The aggregate evidence suggests that if the complete gene clusters were 
captured, many would contain an NRPS module (C-A-T/Cy-A-Ox-T), which may encode thiazole/azole metabolism.

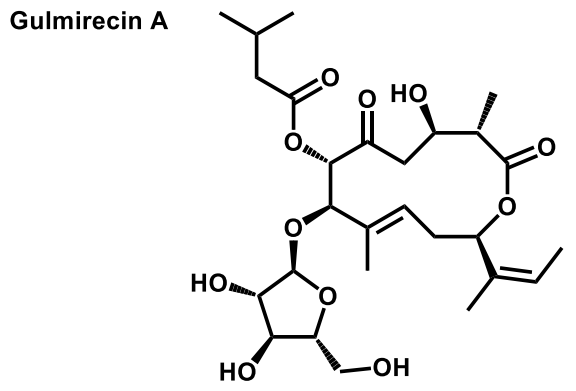

Epothilone A<smiles>CO[C@@H](C[C@@H]1O[C@H]1CCC[C@H](C)[C@H](O)[C@H](C)C(=O)C(C)(C)[C@@H](O)CC(=O)O)/C(C)=C/c1csc(C)n1</smiles>

Cystothiazole A<smiles>COC(=O)/C=C(/OC)[C@H](C)[C@H](/C=C/c1csc(-c2csc(C(C)C)n2)n1)OC</smiles>

Stigmatellin A<smiles>C/C=C(C)/C=C/C=C/[C@H](OC)[C@@H](C)[C@H](OC)[C@@H](C)CCc1oc2c(O)c(OC)cc(OC)c2c(=O)c1C</smiles>

Ajudazole A<smiles>C=C(C/C=C/C=C/CC/C=C/CN(C)C(=O)/C=C(\C)OC)c1nc(C[C@H](C)[C@H]2OC(=O)c3c(ccc(C)c3O)[C@H]2O)co1</smiles>

Ambruticin<smiles>CC[C@H]1O[C@@H](/C(C)=C/[C@H](C)/C=C/[C@@H]2[C@@H](/C=C/[C@@H]3O[C@H](CC(=O)O)C[C@H](O)[C@H]3O)[C@@H]2C)CC=C1C</smiles>

Chlorizidine A<smiles>O=C1c2c(cc(O)c([C@@H]3CCc4cc(Cl)c(Cl)n43)c2O)-c2cc(Cl)c(Cl)n21</smiles>

Phenalamide A1<smiles>CC(/C=C/C=C/C=C/C=C(\C)C(=O)NC(C)CO)=C\C(C)C(O)/C(C)=C/C(C)CCc1ccccc1</smiles>

Myxalamid A<smiles>CCC(C)/C=C(\C)[C@H](O)[C@@H](C)/C=C(C)/C=C/C=C/C=C/C=C(\C)C(=O)N[C@@H](C)CO</smiles>

Figure 4.11 - Known compounds produced by biosynthetic gene clusters with homology to those in GCF_01980. Compounds depicted are the results of biosynthetic pathways that were indicated by antiSMASH clusterBLAST feature to be the "Most similar" biosynthetic gene cluster to at least one gene cluster in GCF_01980. Additionally, gulmirecin was included in the gene cluster family when constructed using BiG-SCAPE. 
The other class of biosynthesis that antiSMASH clusterBLAST highlights as having similarity to some of the members of GCF_01980 are partially cyclised type I PKSs (stigmatellin, ambruticin, phenalamide - Figure 4.11), which are more similar to the gulmirecin biosynthetic gene cluster BiG-SCAPE groups in to GCF_01980.

This evidence, taken as a whole, indicates GCF_01980 has the basis for the biosynthesis for a range of long polyketide compounds. Many of these likely contain at least one amino acid derived motif, potentially a thiazole/azole (evidenced by the C-A-T/Cy-A-Ox-T domains, and the presence of $\mathrm{mbtH}$ in other clusters). The range of cis and trans acting tailoring genes/domains found in all members of this gene cluster family (DH-, ER-, cMT, oMT-, Cy-, Ox-, free TE, mbtH, sulfotransferase) provides further evidence for the production of highly specialised metabolites, which could have interesting biological functions. GCF_01980 is a highly modified family of polyketides that is present across all four of the New Zealand soil metagenomes screened in this study, although more abundant in the $\mathrm{CH}$ and SL libraries than HM and RX. 


\subsubsection{Gene cluster family 01987}

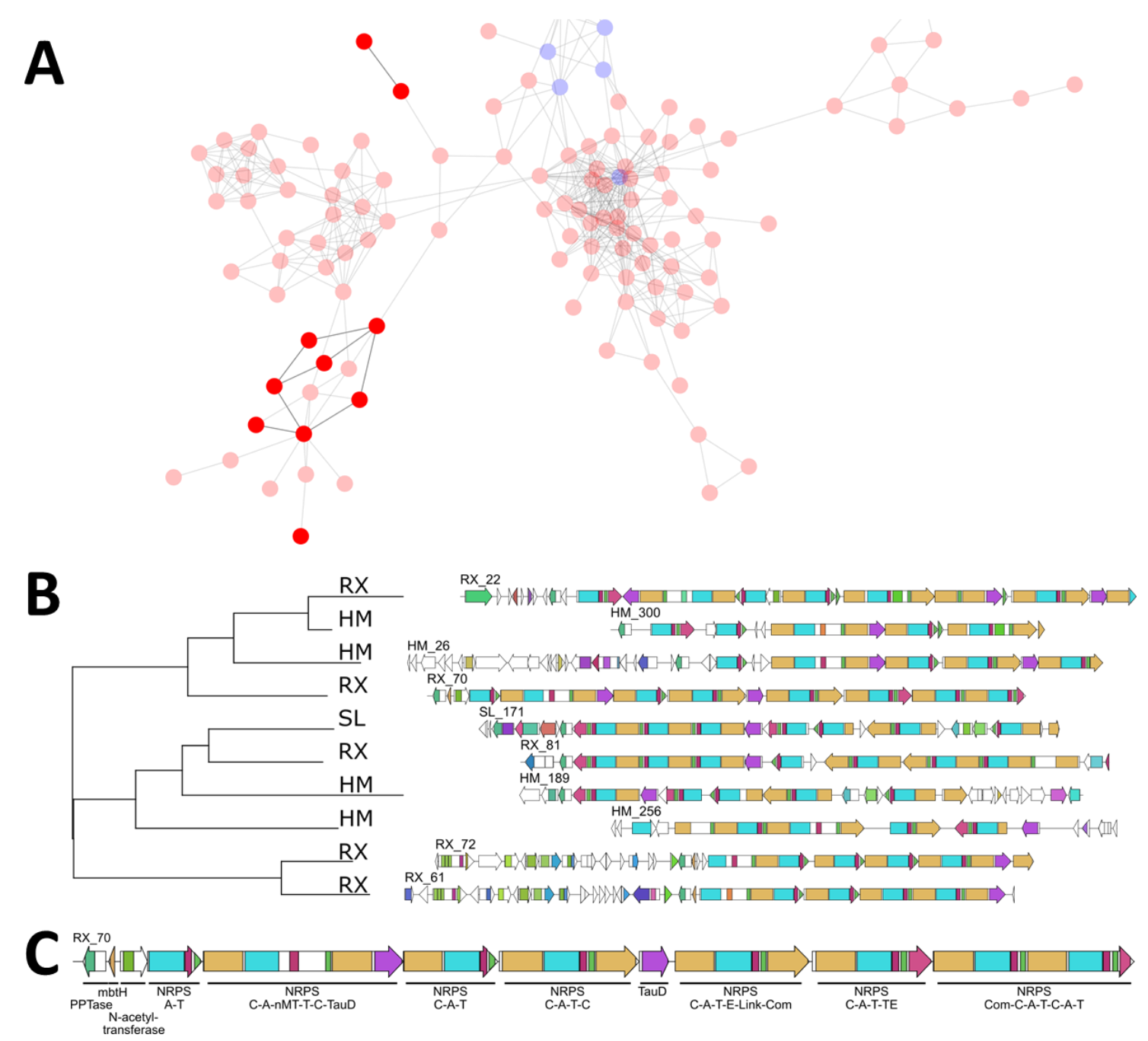

Figure 4.12 - Gene cluster family 01987 (GCF_01987). A) BiG-SCAPE network extracted into Cytoscape, where nodes represent biosynthetic gene clusters from soil metagenomes (red) and MIBiG database biosynthetic gene clusters (blue). The ten nodes belonging to GCF_01987 are highlighted. B) CORASON phylogenetic tree of the biosynthetic gene clusters in GCF_01987. The left graph plots the phylogenetic tree annotated with soil origin of each biosynthetic gene cluster. The biosynthetic gene cluster ORFs are depicted on the right. C) RX_70 biosynthetic gene cluster is shown in detail, with major natural product biosynthesis genes annotated.

GCF_01987 is a family of large, multimodular NRPS biosynthetic gene clusters. Many of the A-domain substrate specificities in this family cannot be accurately predicted by the ensemble of tools included in antiSMASH. Most of the biosynthetic gene clusters contain cis modifying domains, with methyl-transferase FkbM and nMT-domains occurring most frequently. Three biosynthetic gene clusters (HM_300, RX_22, RX_70) contain an NRPS 
module consisting of C-A-T-E-"Link"-Com, where a epimerisation domain is followed by an "inter-modular linker" ${ }^{151}$, and a Com-domain (see Figure 4.12C and Figure 4.14 for RX_70). Inter-modular linkers and Com-domains aid in module-module association of the NRPS biosynthesis pathway. In each case, the C-domain of this module, and that of the subsequent downstream module are characteristic of DCL type C-domains, indicating a Damino acid substrate is condensed into the peptide chain. This arrangement of DCL, E-, and linker-domains suggest the growing peptide is passed to the DCL type C-domain to condense a D-amino acid substrate.

The most notable and distinguishing feature of the GCF_01987 biosynthetic gene clusters are the many instances of genes with pfam annotation as "taurine catabolism dioxygenase (TauD)". These TauD homologue genes are present at least once per cluster (two in RX_70 and three in RX_22). In most cases these are independent ORFs, however six clusters contain TauD homologues C-terminal to an NRPS ORF. In four of these clusters, this NRPS ORF contains a cis MT/FkbH-domain. TauD is a non-heme mononuclear iron hydroxylase enzyme that catalyses the release of sulphite from taurine and other sulfonic acids ${ }^{152}$. TauD homologues have been implicated in NRPS biosynthesis in hydroxylating monomers to $\beta$-hydroxy amino acids. The tailoring function may be catalysed by independent enzymes in biosynthetic gene clusters, or as domains cis to an NRPS cluster, hydroxylating tethered aspartic acid residues to $\beta$-hydroxyaspartic acid ${ }^{44,153}$. Non-heme oxygenases have also been implicated in $\beta$-hydroxylation of asparagine in calciumdependent antibiotics ${ }^{154}$. As all of the TauD-domains in this family are C-terminal linked to an NRPS enzyme, and immediately follow a C-domain, it is likely the TauD domain/enzyme acts to hydroxylate the amino acid activated by the subsequent module. Where biosynthetic gene clusters appear to follow co-linearity, the terminal TauD is always followed by a module selecting Asp or Asn monomers. Free cis acting TauD enzymes are sometimes encoded prior to an Asp/Asn selective module, however these clusters all have two or three Asp/Asn encoding A-domains for potential sites of action if the enzyme does not function co-linear with the gene arrangement.

Analysis of the gene clusters by antiSMASH clusterBLAST revealed low overall identity to any known clusters $(0-60 \%)$. Those that were identified were mostly cyclic peptides/cyclic lipopeptides (Figure 4.13 - microsclerodermins ${ }^{155}$, hapalosin ${ }^{156}$, nostocyclopeptides ${ }^{157}$, puwainaphycins ${ }^{158}$, vioprolide ${ }^{159}$ ). The peptides produced by the biosynthetic gene clusters in GCF_01987 are likely highly modified, with multiple different 
non-proteinogenic amino acids. These gene clusters were found in the three larger metagenomic libraries screened in this study, but were most abundant in HM and RX.

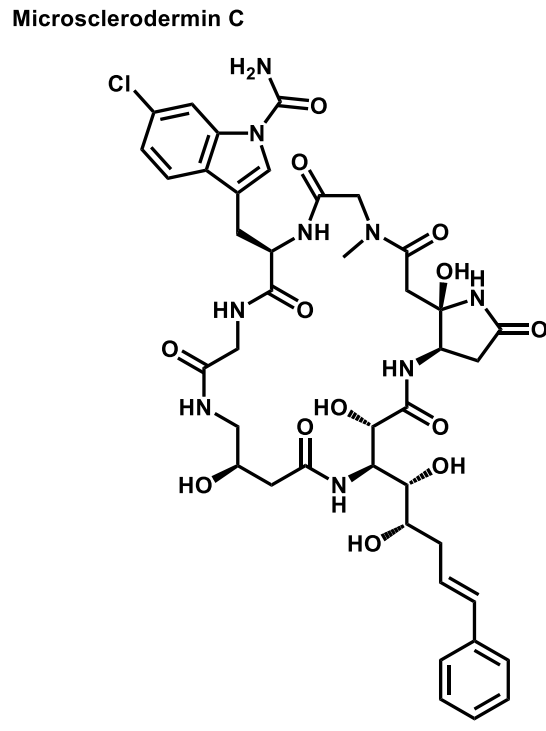

Puwainaphycin A

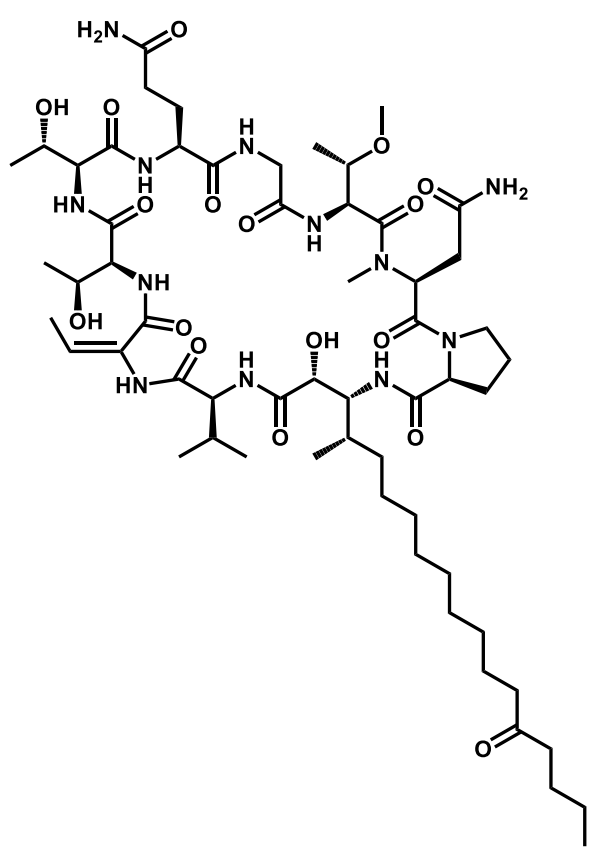

Hapalosin<smiles>CCCCCCC[C@H](OC(=O)C[C@@H](O)[C@H](Cc1ccccc1)N(C)C(=O)[C@H](C)C(C)C)C(=O)O</smiles>

Nostocyclopeptide A1<smiles>CCC(C)NC(=O)C(NC(=O)C(CCC(N)=O)NC(=O)CNC(=O)C(Cc1ccc(O)cc1)N=CC(CC(C)C)NC(=O)C1CC(C)CN1C(=O)C(CO)NC(=O)C(CC)CC)C(C)CC</smiles>

Vioprolide

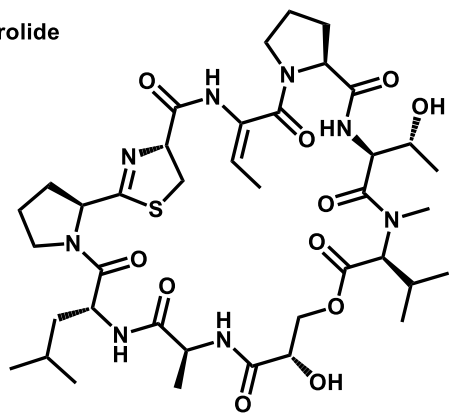

Figure 4.13 - Known compounds produced by biosynthetic gene clusters with homology to those in GCF_01987. All compounds depicted are the results of biosynthetic pathways that were indicated by antiSMASH clusterBLAST feature to be the "Most similar" biosynthetic gene cluster to at least one gene cluster in GCF_01987. 
A)

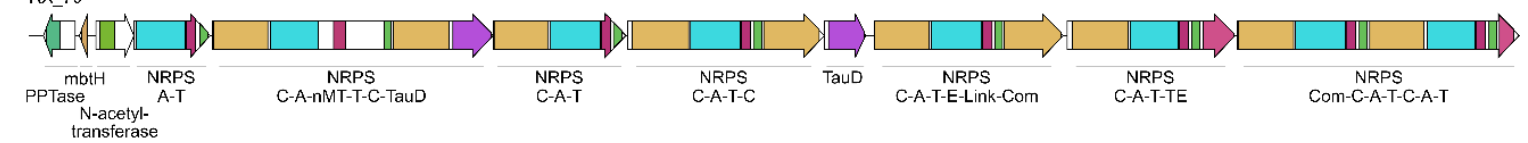

B)
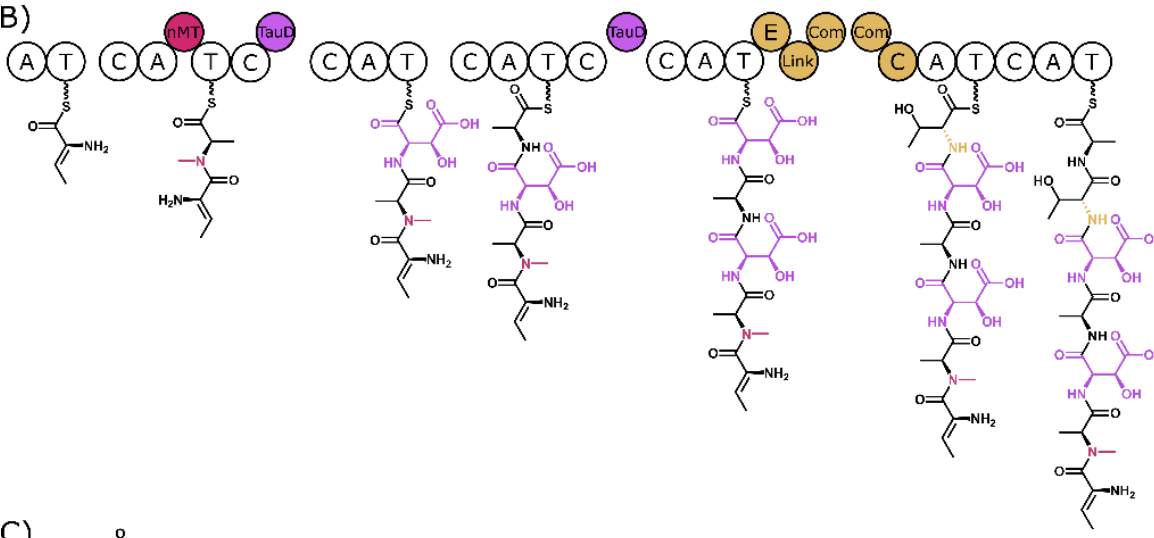

(C) A(P (T)

C)
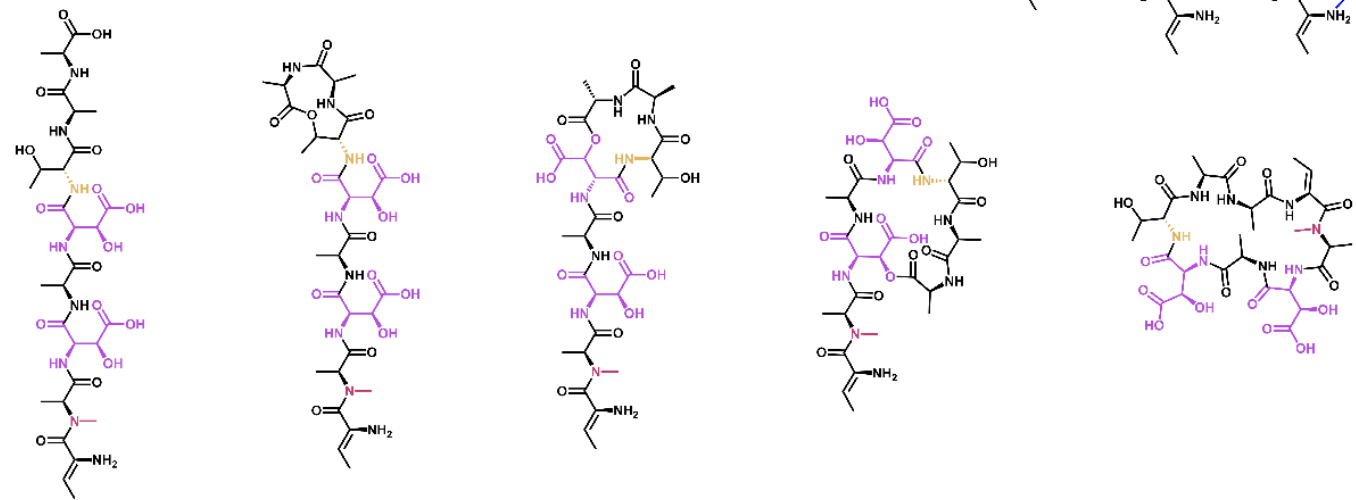

Figure 4.14 - Speculative biosynthesis scheme for RX_70, a representative member of GCF_01987. Amino acid specificity for each A-domain as predicted by a consensus of the algorithms in antiSMASH v4. Tailoring domains and their functions are coloured for emphasis; $\mathrm{N}$-methyltransferase (red), TauD type monooxygenase (purple), epimerisation domain (yellow). TauD enzymes are shown to function in $\beta$-hydroxylation of the amino acid activated by the subsequent module (in both cases on aspartic acid). The final peptide may be released linearly, or cyclised with one four possible nucleophile groups present on the structure (the five blue arrows indicate all possible mechanisms for hydrolysis. C) The five potential molecules of each possible TE-domain release mechanism.

A proposed biosynthesis mechanism for RX_70 is illustrated in Figure 4.14-B. This scheme follows NRPS co-linearity principles with the exception of the final two NRPS ORFs being in opposite order, to link the C-terminal and N-terminal Com-domains together. This order of biosynthesis also places the NRPS module containing the TE-domain as the terminal unit. In the proposed biosynthesis, the first alanine residue is N-methylated, and both aspartic acid residues are $\beta$-hydroxylated by TauD to form $\beta$-hydroxyaspartic acid. The first aspartic acid is modified by a TauD-domain at the C-terminal end of the second NRPS ORF. The second aspartic acid is modified by a free TauD enzyme, which is transcribed co-linearly between the fourth and fifth NRPS ORFs. Five potential peptide structures are 
provided in Figure 4.14-D, with the TE-domain either releasing a linear peptide, or catalysing cyclisation with any of the four available nucleophile attacking groups $(\mathrm{N}$ terminal amine, $\beta-\mathrm{OH}$ of Thr or either of the two $\beta-\mathrm{OH}-\mathrm{Asp}$ ), to form a cyclic peptide or peptidolactone. These mechanisms have been previously observed, with the $\beta-\mathrm{OH}$ of standard Thr used as a nucleophile for cyclisation in daptomycin ${ }^{154,160}$, and modified $\beta$ hydroxylated amino acids used in peptidolactone formation in ramoplanin ( $\beta$-OH-Asn) 45,154. The N-acetyl transferase encoded within the RX_70 biosynthetic gene cluster is not shown to act here, as acetyl transferases often function as resistance elements, rather than in biosynthesis ${ }^{161}$. However, if the $\mathrm{N}$-acetylation of the terminal amine were part of the biosynthesis mechanism, this would block the "head-to-tail" peptide cyclisation, leaving only the $\beta-\mathrm{OH}$ residues available for macrocylisation via the peptidolactone.

\subsection{Discussion}

The aim of the experiments and analysis described in this chapter was to enrich the metagenome libraries described in Chapter 3 for secondary metabolism biosynthesis, and then to examine those biosynthesis genes to explore the "landscape" for bacterial secondary metabolism in New Zealand soils. In particular, I sought to discern whether the secondary metabolic profile of soil microbiomes had been effectively captured in the subset of bacteria that are readily cultivable, and whether new biosynthetic patterns could be uncovered by examining non-cultivated bacterial sources. Based on previous studies into the untapped potential of non-cultivated bacteria, we hypothesised that these metagenome libraries would contain novel biosynthesis pathways that could produce novel chemistry.

Direct sequencing of soil metagenomes is still a significant technical challenge, and requires a large investment in generation of raw sequencing data and subsequent computation. To get around this problem, we employed the $\Delta e n t D$ PPTase selection process to enrich four large soil metagenome libraries for natural product biosynthetic gene clusters. In total, $\sim 5000$ colonies were picked from this screening approach, leading to the discovery of 503 metagenome-derived biosynthetic gene clusters, 427 of which were NRPS and/or PKS systems (Table 4.2). By way of comparison, the largest metagenome sequencing project reported to date uncovered 326 partial and complete NRPS and PKS biosynthetic gene clusters ${ }^{16}$ (however, many other clusters of different metabolic classes were also uncovered in that study). Future investigations can potentially make use of these gene 
sequences and the analysis in this chapter to recover the full gene clusters from these libraries, and use heterologous expression (as applied in Chapter 5 and Chapter 6) to produce bioactive metabolites from these biosynthetic gene clusters.

The gene products encoded in the biosynthetic gene clusters from each library primarily exhibited $<50 \%$ identity to known biosynthetic gene cluster protein sequences (Table 4.4 , Figure 4.3). This indicates that the biosynthetic gene clusters discovered in the course of this work are evolutionarily distinct from previously studied biosynthetic gene clusters. It is therefore likely that these gene clusters have the potential to produce unique metabolites, which could be used as lead compounds to generate new drugs in the future.

The gene products from each library, likewise, exhibited low homology to sequences from all the other libraries (Figure 4.3). This demonstrated that there is potential for unique metabolism in different environmental samples gathered, and therefore potentially a very large resource of biosynthetic diversity that has not been previously studied. Also demonstrated by this analysis was that some metabolism is more conserved between different metagenome samples. This implies that common clades of microbial life present across multiple environments produce novel metabolites, and are not accessible to cultivation-based discovery methods. Taxonomic analysis of the gene products encoded in these biosynthetic gene clusters further supports this conclusion, as the majority of the gene products classify into bacterial phyla that are underrepresented in the study of natural product biosynthesis (e.g. Acidobacteria, Proteobacteria - Figure 4.2, Table 4.3, Figure $4.6)$.

By examining the gene cluster families found in common between the New Zealand soil metagenomes, some interesting collections of related secondary metabolism pathways were uncovered (GCF_01965, GCF_01980, GCF_01987). These three families were also demonstrated to have their likely taxonomic origins in Gram-negative bacterial phyla Acidobacteria and Proteobacteria (Figure 4.6). In each case, these contained multiple biosynthetic gene clusters without precedence in previous studies. Because of this, it was not always possible to make fully informed predictions about the natural products that could result from these pathways. Nonetheless, in each case some hypotheses were made, and comparisons drawn to known, bioactive and valuable biosynthesis pathways. The analyses revealed numerous "hidden" secondary metabolism patterns, that are seemingly abundant in the soil microbiome and rare or entirely absent in cultured bacteria. 
GCF_01965 was speculated to encode biosynthesis mechanisms for a polyglycosylatedcyclodipeptide. To my knowledge there are no explicit examples of such structures in published literature. However, extensive genome mining of non-NRPS tRNA-derived cyclodipeptide containing biosynthetic gene clusters from over 93,000 bacterial genomes did find evidence of deoxy sugar biosynthesis and glycosyltransferase genes associated with cyclodipeptide biosynthetic gene clusters ${ }^{146}$. The authors of that study suggested this indicates some clusters do produce glycosylated cyclodipeptides, albeit from a different biosynthetic mechanism than proposed in section 4.7.1.

GCF_01980 is a collection of incomplete biosynthetic gene clusters that likely encode hybrid type I PKS-NRPS biosynthesis, potentially producing macrolide polyketide molecules with thiazole residues. Known metabolites from these types of biosynthesis pathways have interesting and valuable antibiotic and anticancer bioactivities. For example, multiple epothilone derivatives have been explored in clinical trials as anticancer agents stabilising microtubules ${ }^{162}$.

GCF_01987 is a family of NRPS biosynthesis pathways that likely produces a diverse set of metabolites. The peptides produced are however likely to be highly modified, with many tailoring functions present throughout the gene clusters, including methylation, epimerisation, and likely TE-domain catalysed macrocyclisation. The notable abundance of TauD homologues throughout the gene cluster family may indicate a common structural identity or metabolite function. TauD homologues in NRPS biosynthesis are certainly not unprecedented, however the abundance of these gene clusters throughout the four libraries is surprising, and is worthy of further investigation. Beyond GCF_01987, TauD homologues were present in other NRPS gene cluster families, including two biosynthetic gene clusters in GCF_01965. Other metagenome gene cluster families contained abundances of TauD homologues on a level similar to GCF_01987, including GCF_01850, GCF_01985,GCF_02018, and GCF_02164.

Taxonomic analysis of the genes products uncovered in this pipeline revealed a markedly different distribution of bacterial phyla in each of the soil metagenome libraries as compared to the biosynthetic gene clusters present in the MIBiG database (Figure 4.2). As 98\% of the entries in MIBiG originated with cultured bacteria/fungi, it is reasonable to conclude that the bias present in this dataset is a result of discovery pipelines being almost exclusively reliant on culturable bacteria. Many of these culture-based discovery pipelines 
also use media selective for actinomycetes (e.g. actinomycete isolation agar AIA) and other Gram-positive bacteria by the inclusion of nalidixic acid in the agar media. The majority of the biosynthetic diversity of each of the soils examined in this study cannot be accessed in these workflows, being clades not commensurate with culture on selective media, or Gram-negative species killed off by selective antibiotics commonly used. It seems this is a self-perpetuating bias - a study by Katz and Baltz into 100 important and biosynthetically diverse microbial produced small molecules showed $83 \%$ were produced by actinomycetes, $12 \%$ by fungi, and $5 \%$ by "other bacteria" ${ }^{6}$. Researchers target actinomycete secondary metabolites because of historical successes ${ }^{69}$, however a growing body of evidence (including the results of this study) may provide reason to encourage diversification of efforts. Recent surveys into bioactive metabolite production of non-Streptomyces actinomycetes (e.g. Nocardia spp. ${ }^{71}$ ) and non-actinomycete taxa (e.g. Myxobacteria ${ }^{70}$ ) have revealed not just an abundance of biosynthetic capacity, but a potentially nonoverlapping set of metabolite diversity relative to highly studied clades. By looking further outwards towards different bacterial phyla emphasised in section 4.6.1 (e.g. Acidobacteria), it is likely entirely new bioactive metabolites can be found.

This data adds to a growing body of evidence that future success in the bacterial natural products field may be contingent on a shift in focus towards Gram-negative bacteria ${ }^{90}$. However, dedicated discovery platforms based on Acidobacteria culture will require significant optimisation of culture conditions. One study in soil bacteria lab cultivation found diverse soil Acidobacteria species can be cultured on agar media, although colonies only appeared after 12-24 weeks of culture time, with colonies too small to see with the naked eye $(25-200 \mu \mathrm{m}){ }^{163}$. Researchers found colony sizes and growth rates usually increase with subculture however. Others have found significant increases in cultivability of rare bacterial taxa by relatively simple changes in media composition ${ }^{164}$, sonication to separate adhered fast and slow growing species, and use of non-agar gelling agents ${ }^{165}$.

In order to access novel biosynthesis encoded by the biosynthetic gene clusters described in this chapter, heterologous expression is likely to be required ${ }^{91}$. As previously discussed, the dominance of actinomycete study in natural product discovery has provided us with many well characterised heterologous expression hosts ${ }^{73,75-77}$. Likewise, there already exist a number of diverse Proteobacteria heterologous expression systems ${ }^{90,93,94}$. Firmicutes 166,167, and Cyanobacteria ${ }^{168}$ also have expression hosts that have been previously utilised for small molecule production. However, these four phyla only make up 50\% of the 
biosynthetic genes discovered in this study (Table 4.3 - Actinobacteria 6.9\%, Cyanobacteria 3.1\%, Firmicutes $0.4 \%$, Proteobacteria 39.6\%). Acidobacteria are a major component of the soil microbiome ${ }^{163,164,169}$, and also a major contributor to the secondary metabolism of the soil (this study, and Crits-Christoph et al. $2018^{16}$ ). If Acidobacterial biosynthetic gene clusters could be shown to express within validated Gram-negative expression hosts, or an appropriate host developed, a further $29.9 \%$ of the biosynthetic potential of the soils from this study could be accessed (bringing the total to almost $80 \%$ ). A more well developed set of expression systems would also provide access to the secondary metabolism of other Gram-negative phyla uncovered in this study - including Verrucomicrobia (7.8\% of total metagenome), Planctomycetes (5.8\%), Nitrospirae (1.6\%), Gemmatimonadetes (1.6\%), and Bacteroidetes (1.5\%) - approaching potential full access of the secondary metabolism in the collected sequence data.

All of the cosmid metagenome libraries examined in this study contained novel natural product biosynthesis pathways. This is an encouraging finding for the study of natural product biosynthesis, indicating that there are many environments possessing undiscovered natural product biosynthetic capacity. 


\subsection{Type II polyketide biosynthetic gene cluster recovery and metabolite characterisation}

\subsection{Summary}

Aromatic polyketides are produced by a conserved core set of proteins in type II PKS biosynthesis $-\mathrm{KS}_{\alpha}, \mathrm{KS}_{\beta}$ and an $\mathrm{ACP}$ (section 1.3.2). The abundance of conjugated pi-bonds in these metabolites often results in visibly coloured compounds, facilitating their detection in heterologous expression systems.

Another member of the research lab group (Dr. Liwei Liu) had screened the HM cosmid metagenome library described in Chapter 3 with degenerate primers, and recovered 29 cosmids containing $\mathrm{KS}_{\alpha}$ genes. In this chapter, I describe taking one of these cosmids (D9), sequencing the metagenome insert, and characterising the genetic pathway it contains. I performed heterologous expression studies of this biosynthetic gene cluster in S. albus $\mathrm{J} 1074$, resulting in the production of at least three compounds of interest. One major product was isolated, purified, and its structure elucidated using a combination of highresolution mass spectrometry (HR-MS) and 1 dimensional and 2 dimensional NMR experiments. This analysis revealed that the major product of the pathway (compound $\mathbf{1}$ ) was new, and in bioactivity studies against a diverse panel of test strains it possessed weakmoderate Gram-positive and -negative antibacterial activity, and anticancer cytotoxicity.

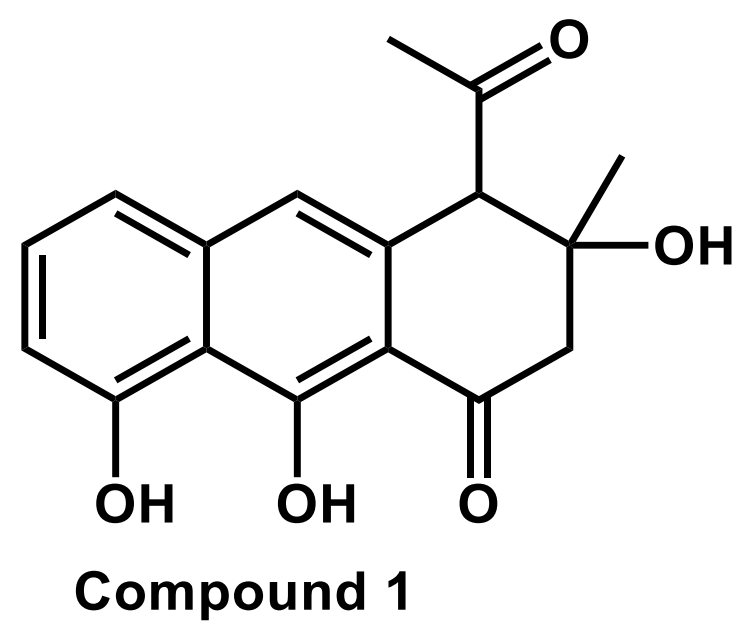




\section{2. $\mathrm{KS}_{\boldsymbol{\alpha}} \mathrm{PCR}$ screening}

The Half Moon Bay cosmid metagenome library (HM) that was described in Chapter 3 was screened by Dr Liwei Liu using degenerate primers for the conserved $\mathrm{KS}_{\alpha}$ gene ${ }^{85}$, required for type II polyketide biosynthesis (Figure 5.1). Type II polyketides originate from iterative biosynthetic systems, where acyl/malonyl-CoA monomers are added to the growing polyketide chain, increasing the carbon chain length 2 carbon units with each cycle ${ }^{18,42}$. When the growing polyketide fills the synthesis pocket, the polyketide product is released, and can be modified, typically by cyclase, aromatase and ketoreductase enzymes to yield aromatic compounds ${ }^{18,42}$. Further modifications that are common in yielding a mature bioactive molecule include: methylation, glycosylation, oxygenase catalysed rearrangements, halogenation and reduction ${ }^{42,43}$. The 29 cosmids that were recovered from the HM library were sequenced en masse by Illumina HiSeq $2500100 \mathrm{bp}$ PE sequencing, and then retrofitted with a fragment from a DraI digest of pOJ436 that contained an origin of transfer (OriT), an integrase and an apramycin resistance cassette $\left(\mathrm{Apr}^{\mathrm{R}}\right)$, prior to conjugation into $S$. albus. I then subjected the resulting strains to further analysis.

Two cosmids were chosen for further study: One cosmid (D3) contained a partial gene cluster for an aureolic acid type pathway, as determined by the initial DNA sequencing results and subsequent antiSMASH analysis. The aureolic acids are very potent class of anticancer antibiotics, and there is a lot of interest in the natural product field to find new congeners of this class ${ }^{49,170,171}$. There are also only two known biosynthetic gene clusters for aureolic acids, therefore a new biosynthetic pathway would constitute a relevant contribution to the research field. Initial sequencing of the cosmid containing this pathway resolved to a single contig, which was analysed by antiSMASH 4.0 to show a high cluster homology to the two known aureolic acid biosynthetic gene clusters. However, on an individual gene level, homology was low, and significant divergence in gene arrangement was apparent. The cluster was obviously incomplete, with a truncated ORF that had homology to a required resistance element in aureolic acid biosynthesis. For this reason, significant genetic reconstitution of the pathway was required, and examination of this pathway formed the basis of Chapter 6. 


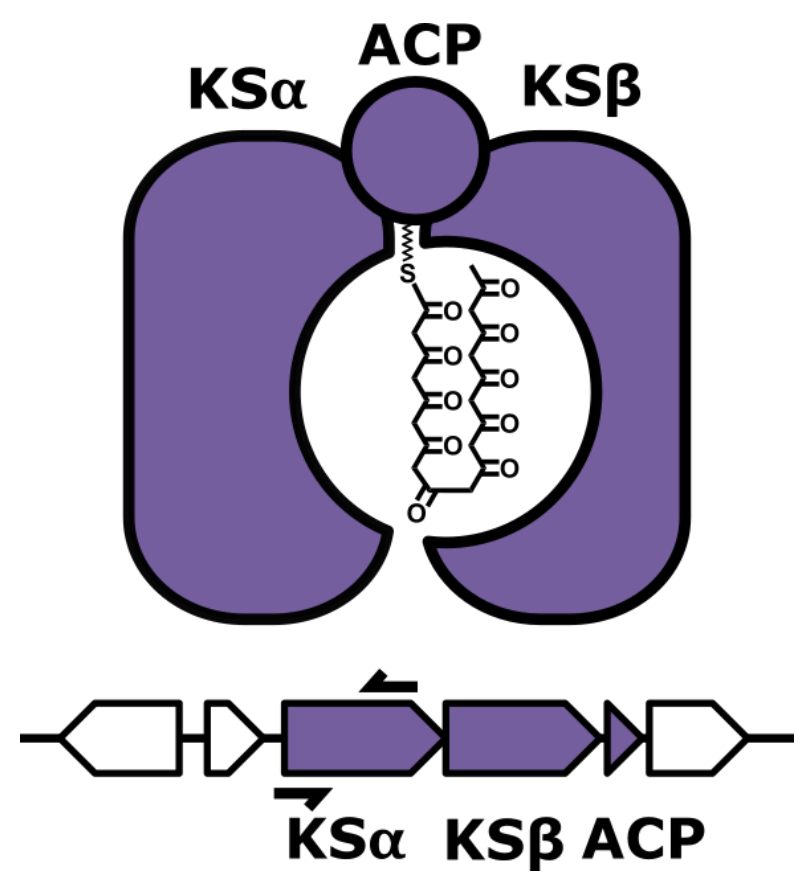

Figure 5.1 - Type II polyketide biosynthesis degenerate primer screening. The core PKS unit is depicted, showing the growing polyketide chain tethered to the acyl carrier protein domain (ACP), and filling the synthesis pocket. The $\mathrm{KS}_{\alpha}$ domain is thought to catalyse the synthesis via successive condensation reactions, while the $\mathrm{KS}_{\beta}$ domain regulates the number of addition cycles (also known as the "chain length factor"). The degenerate screening primers used in this study were based on a conserved region of the $\mathrm{KS}_{\alpha}$ gene ${ }^{85}$.

Another cosmid (D9) contained an apparent angucycline type biosynthesis pathway, and when conjugated into $S$. albus produced a large amount of what initially appeared to be two coloured metabolites; one green and one purple. Preliminary testing indicated the green metabolite had antibiotic activity against $B$. subtilis 168 . Initial sequencing of the D9 cosmid could not assemble a full length contig with any assembly program used. Investigation into this genetic pathway continued however, not on the basis of genetic information, but as a result of the large amount of polyketide compound being produced from heterologous expression in the $S$. albus culture.

\subsection{Initial expression studies}

Heterologous expression of the pathway was conducted at $30{ }^{\circ} \mathrm{C}$ in $\mathrm{R} 5$ a medium, using an inoculum of $1 \%$ mature S. albus D9 TSB culture. Expression cultures were fermented for 7-10 days at $30{ }^{\circ} \mathrm{C} 200 \mathrm{rpm}$, with culture medium generally taking on a dark green/brown colour after 3-4 days of fermentation. Following fermentation, compound extraction from 
the culture was performed. Initial trials (Figure 5.2) were performed by bulk ethyl acetate extraction of the culture medium, where the medium was combined $1: 1 \mathrm{v} / \mathrm{v}$ with ethyl acetate, mixed, and the upper organic phase recovered followed by complete solvent removal in vacuo.
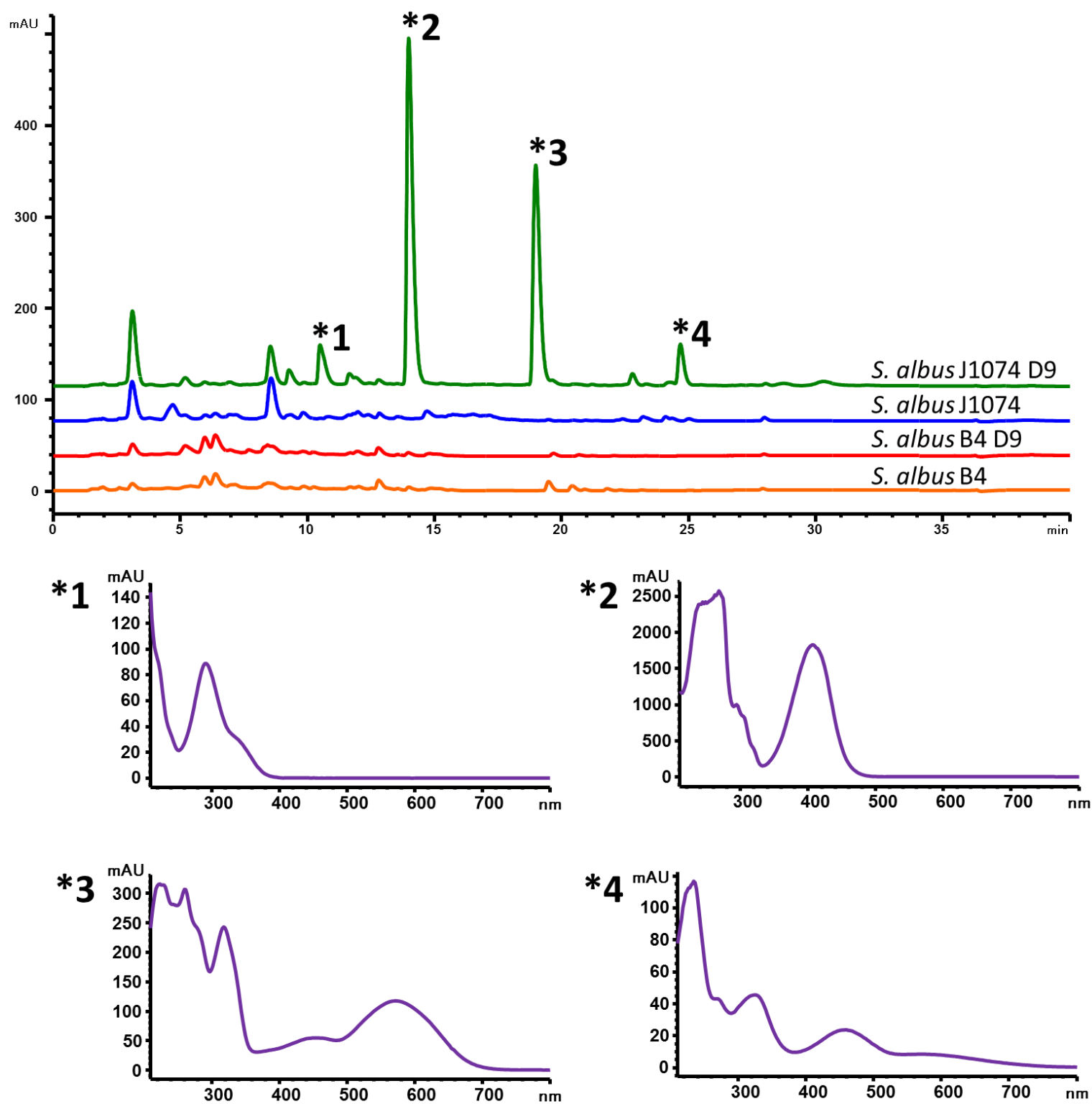

Figure 5.2 - HPLC trace of S. albus D9 culture extract. The $S$. albus background extract (minus pathway) is shown in blue, and S. albus with the D9 metagenome biosynthetic pathway in green. Alternative expression system S. albus B4 extract traces are in orange (minus pathway) and red (with D9 biosynthetic pathway). The retention time on a reversedphase $\mathrm{C}_{18}$ column is shown on the $\mathrm{X}$-axis, and absorbance at $320 \mathrm{~nm}$ on the $\mathrm{Y}$-axis. The major metabolites present in the pathway positive extract are indicated by an asterisk (*), with corresponding UV absorbance spectra depicted below. 
Four new peaks were observed on the HPLC trace of the $S$. albus: $: D 9$ culture relative to an S. albus control (Figure 5.2). Peak 2 is the green product of fermentation, and peak 3 is the purple product. Neither peak 1 nor 4 presented as coloured compounds. In preliminary bioactivity assays, where the HPLC-isolated peaks 1-4 were spotted onto LB agar infused with test strain B. subtilis 168 , peak 2 possessed significant antibiotic activity, which was not observed with any other isolated peak. For this reason, compound isolation efforts were focussed on the metabolites of peak 2 .

During the course of studying this pathway, a "zero background" strain of S. albus J1074 was produced by Andriy Luzhetskyy's research group, in which the 15 native biosynthetic gene clusters had been removed ${ }^{77}$. Alongside the gene deletions, the researchers had introduced additional $a t t B$ integration sites, to increase the copy number of introduced gene clusters for heterologous expression (S. albus B4). However, extracts of this producing strain expressing the cosmid metagenome pathway contained only trace amounts of the peak 2 associated metabolites (Figure 5.2). This strain was not used going forward.

In order to identify the best method for scaled up compound isolation, HP20 resin based extraction of the culture medium, and solvent extraction from dry cell mass were compared to bulk ethyl acetate extraction. $10 \mathrm{~mL}$ of a mature $\mathrm{R} 5 \mathrm{a}$ culture was extracted $1: 1$ with ethyl acetate, and the organic phase separated. A second $10 \mathrm{~mL}$ of mature R5a culture was then centrifuged to separate a cell pellet from cell-free supernatant. The supernatant was added to $0.5 \mathrm{~g}$ of HP20 resin, and shaken for 2 hours to allow the organic compounds to adsorb to the resin. The resin was collected, and eluted first with methanol, then acetone. Meanwhile, the cell mass recovered from the centrifugation step was extracted first with methanol, and then with acetone. The five extracts were dried in vacuo, and then resuspended in methanol or acetone, and the extraction methods assessed by HPLC, where the integration of peaks was used to measure the recovery of each product. 


\begin{tabular}{lllll}
\hline & \multicolumn{3}{l}{ HPLC peak } & \\
& $\mathbf{1}$ & $\mathbf{2}$ & $\mathbf{3}$ & $\mathbf{4}$ \\
\hline Retention time (min) & 10.5 & 14 & 19 & 25 \\
\hline Ethyl acetate extract & 679.1 & 1605 & 1190.4 & 661.2 \\
HP20 methanol elution & 50.7 & 929.9 & 179.2 & - \\
HP20 acetone elution & - & 676.8 & 182.4 & - \\
Cell pellet methanol extract & 33.8 & 71.1 & 231.6 & 1165 \\
Cell pellet acetone extract & - & - & - & 25.16
\end{tabular}

Table 5.1 - Metagenome D9 pathway metabolite extraction methods. Identical expression cultures in R5a medium were extracted using the stated methods, and the extracts analysed by HPLC. The major identified pathway associated metabolite peaks on the HPLC trace were integrated to compare extraction yields under the various conditions.

The ethyl acetate extraction method was successful in recovering the greatest amounts of peak 2 and 3 (Table 5.1). While HP20 extraction was able to recover similar quantities of peak 2, it did not provide similar recovery of the other products. From these results, it is clear the vast majority of peak 2 metabolites are present in the culture media, potentially indicating active metabolite export from the production strain. HP20 compound extraction from culture media produced "dirtier" extracts than ethyl acetate extraction, as assessed by weight of crude extract, indicating non-specific adsorption of culture media components to the resin. For these reasons, ethyl acetate extraction was used as the primary extraction method. Further empirical testing of extraction conditions demonstrated that ethyl acetate extraction was not improved under acidified conditions ( $\mathrm{pH} 2-3$ ), with secondary extraction on acidified aqueous phase yielding $50.4 \%$ of the integration of peak 2 recovered in primary neutral phase extraction. The results suggested that that the compound(s) in peak 2 do not possess acidic functional group(s) that would require protonation under acidified conditions for ethyl acetate extraction. Neutral ethyl acetate extraction was therefore used for metabolite extraction from expression cultures.

LC-MS analysis of the ethyl acetate culture extract was unable provide an accurate mass for peaks 1 and 4. Investigation into the compounds associated with these peaks was halted here, as polyketide compounds in general are easily ionisable in negative ion mode and no indication of antibiotic activity had been observed for these compounds. Peak 2 was found to contain two ionisable metabolites, corresponding to deprotonated molecules with 
negative $\mathrm{m} / \mathrm{z}$ of 299.0917 (minor) and 323.0919 (major). Peak 3 contained a single metabolite with a negative $\mathrm{m} / \mathrm{z}$ of 319.0623 .

For clarity, observed masses will hereafter be referred to as compound $\mathbf{1}$ (peak 2 minor constituent with m/z 299.0.917 [M-H]'), compound 2 (peak 2 major constituent with $\mathrm{m} / \mathrm{z}$ 323.0.919 [M-H] $]^{-}$), and compound 3 (peak 3 m/z $319.0623[\mathrm{M}-\mathrm{H}]^{-}$).

\subsection{Fermentation and compound isolation}

Initial compound isolation experiments focussed on recovery of compounds $\mathbf{1}, \mathbf{2}$, and $\mathbf{3}$. For prefractionation of the crude ethyl acetate extracts, normal phase chromatography and size exclusion chromatography were employed separately.

A benchtop normal phase silica column was used to separate the crude ethyl acetate extract of a mature R5a expression culture. The silica $(10 \mathrm{~mL})$ was equilibrated with chloroform $(0.1 \%$ acetic acid), and ethyl acetate extract from $1 \mathrm{~L}$ of culture was loaded onto the column in the same solvent. The column was then eluted with $80 \mathrm{~mL}$ chloroform $(0.1 \%$ acetic acid $)$ followed by $80 \mathrm{~mL} 2 \%$ methanol/chloroform ( $0.1 \%$ acetic acid). The major metabolites coeluted in $2 \%$ methanol/chloroform $(0.1 \%$ acetic acid) as a dark green band that was collected in bulk. Remaining bound metabolites were eluted in $4 \%$ and $8 \%$ methanol chloroform ( $0.1 \%$ acetic acid) elutions ( $80 \mathrm{~mL}$ volumes each). Fractions were assessed by HPLC, which demonstrated that the majority of compounds 1-3 had been collected in the single fraction. With MS analysis following semipreparative HPLC purification, it became apparent the majority of compound $\mathbf{2}$ had degraded, and was only minimally detected in downstream purification fractions, whereas compound $\mathbf{1}$ appeared to remain stable.

Size exclusion chromatography using LH20 resin had the same effect, where compound 2 degraded in the process of purification, while compound 1 remained stable and was readily recovered. Compound $\mathbf{3}$ was also isolated from both of these procedures, however in very small quantities. Compound $\mathbf{3}$ is a strong purple pigment, facilitating ready detection, however upon isolation was found to be low in abundance. When isolated, compound 3 quickly precipitated out of solution, and was not sufficiently soluble in any NMR solvent to acquire usable data.

As compound 2 was produced in culture at high abundance, further attempts at isolation were conducted. The most successful was achieved by combining the ethyl acetate and 
HP20 extraction methods. The cell free supernatant from $5 \mathrm{~L}$ of $S$. albus D9 fermentation in R5a was recovered by centrifugation. The $5 \mathrm{~L}$ of supernatant was then run onto $200 \mathrm{~mL}$ of HP20 in a benchtop column to bind the metabolites to the resin. This was then washed successively with $1 \mathrm{~L}$ of $\mathrm{H}_{2} \mathrm{O}$ and $1 \mathrm{~L} 20 \%$ acetone/ $\mathrm{H}_{2} \mathrm{O}$ to remove polar metabolites and media components (sugars, amino acids etc.). The target metabolites were eluted from the $\mathrm{HP} 20$ in $1 \mathrm{~L}$ of $80 \%$ acetone $/ \mathrm{H}_{2} \mathrm{O}$, which was collected in bulk. Roughly half of the solvent volume of the $80 \%$ acetone $/ \mathrm{H}_{2} \mathrm{O}$ elution was removed under reduced pressure, and the sample was brought back up to $1 \mathrm{~L}$ volume with $\mathrm{H}_{2} \mathrm{O}$, to achieve a more polar solvent mixture permissible to partitioning with ethyl acetate. The $1 \mathrm{~L}$ sample was extracted twice with $1 \mathrm{~L}$ ethyl acetate, and the organic phases were pooled and dried completely under reduced pressure. From a small sample of this extract, $6.0 \mathrm{mg}$ of compound $\mathbf{2}$ was recovered by semipreparative $\mathrm{C}_{18}$ HPLC, with a linear gradient from $30-50 \%$ acetonitrile $/ \mathrm{H}_{2} \mathrm{O}$ over 30 minutes (Phenomenex $10 \mathrm{~mm} \times 250 \mathrm{~mm}, 4 \mathrm{~mL} / \mathrm{min}, 25^{\circ} \mathrm{C}$ ), where compound 1 had a retention time of 18.1 minutes, and compound 2 had a retention time of 17.8 minutes.

\subsection{Structure elucidation}

From the compound isolation efforts, sufficient quantities of the two green (peak 2) associated metabolites - compounds $\mathbf{1}$ and $\mathbf{2}$ - were recovered for NMR studies. NMR experiments were performed in collaboration with $\mathrm{PhD}$ candidate Joe Bracegirdle from Victoria University of Wellington's School of Chemical and Physical Sciences.

Although the final attempted extraction method recovered a large amount of compound 2 , stability issues continued to hamper structure elucidation. Compound $\mathbf{2}$ was detected by high-resolution mass spectrometry in negative mode at $\mathrm{m} / \mathrm{z} 323.0919[\mathrm{M}-\mathrm{H}]^{-}$and in positive mode at $\mathrm{m} / \mathrm{z} 325.1072[\mathrm{M}+\mathrm{H}]^{+}$, consistent with a molecular formula $\mathrm{C}_{19} \mathrm{H}_{16} \mathrm{O}_{5}$ (predicted $[\mathrm{M}+\mathrm{H}]^{+}=325.1070,[\mathrm{M}-\mathrm{H}]^{-}=323.0925$ ). This formula indicates a highly unsaturated molecule with hydrogen deficiency index of 12. This made structure elucidation by NMR challenging, with the molecular formula going against "Crews rule" for tractability of NMR characterisation with a ratio of $\mathrm{H}: \mathrm{C}<1{ }^{172,173}$. While the compound was sufficiently soluble in DMSO- $\mathrm{d}_{6}$ to gather high quality NMR data, over the course of these experiments the compound degraded. As this compound degraded on the timescale to collect NMR data, later stage acquisitions had missing data, and this compound structure could not be solved (NMR spectra in Appendix C). 
The indicative molecular formula for compound $\mathbf{3}, \mathrm{C}_{19} \mathrm{H}_{12} \mathrm{O}_{5}\left(\mathrm{~m} / \mathrm{z} 319.0623[\mathrm{M}-\mathrm{H}]^{-}\right.$, $321.0751[\mathrm{M}+\mathrm{H}]^{+}$) differs from compound 2 by a loss of 4 hydrogens. This could be the result of successive reduction or aromatisation of compound 2. Compound $\mathbf{3}$ therefore had an even greater hydrogen deficiency index (14), with solubility and low abundance also inhibiting attempts to gather NMR data.

Compound 1 however was stable, soluble, and abundant and high-quality NMR data was acquired. Accurate masses were obtained in both negative and positive mode: m/z 299.0917 $[\mathrm{M}-\mathrm{H}]^{-}$and $\mathrm{m} / \mathrm{z} 301.1088[\mathrm{M}+\mathrm{H}]^{+}$. This is consistent with a molecular formula $\mathrm{C}_{17} \mathrm{H}_{16} \mathrm{O}_{5}$ (predicted $[\mathrm{M}+\mathrm{H}]^{+}=301.1070,[\mathrm{M}-\mathrm{H}]^{-}=299.0925$ ). Although the formula also indicates a proton deficient molecule (hydrogen deficiency index $=10$ ) that deviates from Crews rule, correlations were observed in the two-dimensional data sets (COSY, HMBC, HSQC) that fully defined the planar structure of the molecule (Figure 5.3, NMR spectra in Appendix B).

The NMR analysis of compound $\mathbf{1}$ was performed with the sample in deuterated chloroform $\left(\mathrm{CDCl}_{3}\right)$. Proton resonance $\left({ }^{1} \mathrm{H}\right)$ and proton decoupled carbon resonance spectra $\left({ }^{13} \mathrm{C}\right)$ were acquired, and the planar structure of the compound was assigned using correlations from two-dimensional NMR techniques: ${ }^{1} \mathrm{H}{ }^{13} \mathrm{C}$ heteronuclear single quantum coherence (HSQC), ${ }^{1} \mathrm{H}^{1} \mathrm{H}$ correlation spectroscopy (COSY), and ${ }^{1} \mathrm{H}^{13} \mathrm{C}$ heteronuclear multiple bond connectivity (HMBC). The ${ }^{1} \mathrm{H}$ and ${ }^{13} \mathrm{C}$ chemical shifts and ${ }^{1} \mathrm{H}$ multiplicities are provided in Table 5.2, and the key correlations from 2D homo/hetero-nuclear NMR experiments overlaid on the structure in Figure 5.3. 


\begin{tabular}{|c|c|c|c|}
\hline Position & $\delta H(p p m)$ & $\begin{array}{l}\text { Multiplicity } \\
\text { (J, Hz) }\end{array}$ & $\delta \mathbf{C}(\mathbf{p p m})$ \\
\hline 1 & & & 202.5 \\
\hline 2 & & & 108.4 \\
\hline 3 & & & 166.8 \\
\hline 4 & & & 113.5 \\
\hline 5 & & & 158.5 \\
\hline 6 & 6.90 & $\mathrm{~d}(7.8)$ & 112.1 \\
\hline 7 & 7.51 & $\mathrm{t}(7.9)$ & 133.2 \\
\hline 8 & 7.17 & $\mathrm{~d}(8.0)$ & 118.5 \\
\hline 9 & & & 139.1 \\
\hline 10 & 7.08 & $\mathrm{~s}$ & 119.4 \\
\hline 11 & & & 132.1 \\
\hline 12 & 4.19 & $\mathrm{~s}$ & 63.1 \\
\hline 13 & & & 71.9 \\
\hline $14 \mathrm{a}$ & 3.50 & $\mathrm{~m}$ & 47.7 \\
\hline $14 b$ & 2.73 & $\mathrm{~m}$ & \\
\hline 15 & & & 205.8 \\
\hline 16 & 2.41 & $\mathrm{~s}$ & 32.2 \\
\hline 17 & 1.48 & $\mathrm{~s}$ & 27.6 \\
\hline $3-\mathrm{OH}$ & 9.77 & $\mathrm{~s}$ & \\
\hline $5-\mathrm{OH}$ & 16.3 & $\mathrm{~s}$ & \\
\hline
\end{tabular}




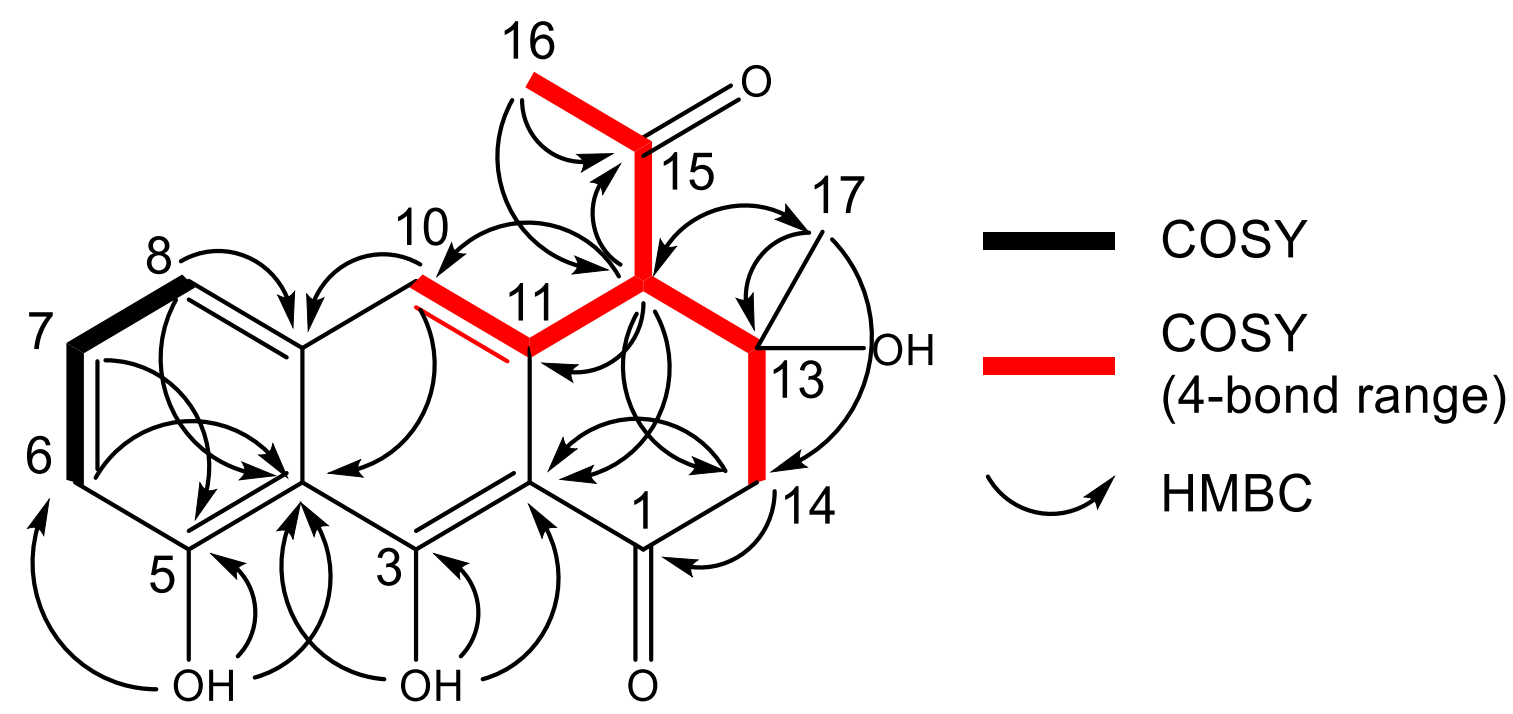

Figure 5.3 - Key NMR correlations by COSY and HMBC for compound 1. Note the long range COSY coupling (red) of H12 to H10, H14b, and H16 exclusively.

The established molecular formula of compound $1\left(\mathrm{C}_{17} \mathrm{H}_{16} \mathrm{O}_{5}\right)$ was consistent with the 17 resonances observed in the ${ }^{13} \mathrm{C}-\mathrm{NMR}$ spectrum. COSY correlations from $\mathrm{H} 6$ and $\mathrm{H} 8$ aromatic doublets $\left(\delta_{\mathrm{H}} 6.90,7.17\right)$ to the $\mathrm{H} 7$ triplet $\left(\delta_{\mathrm{H}} 7.51\right)$ indicated a 1,2,3-trisubstitued aromatic ring. $\mathrm{HMBC}$ correlations from an exchangeable proton $\left(\delta_{\mathrm{H}} 9.77\right)$ to $\mathrm{C} 6\left(\delta_{\mathrm{C}} 112.1\right)$ indicated a phenol at C5 $\left(\delta_{\mathrm{C}} 158.5\right)$, also with correlations to C4 and C6. The first aromatic ring was completed with $\mathrm{HMBC}$ correlations around the ring structure, extending into the adjacent ring, where a second exchangeable proton $\left(\delta_{\mathrm{H}}\right.$ 16.30) displayed HMBC correlations with $\mathrm{C} 4$ and $\mathrm{C} 2\left(\delta_{\mathrm{C}} 113.5,108.4\right)$, indicating a second phenol at $\mathrm{C} 3\left(\delta_{\mathrm{C}} 166.8\right)$. The $\mathrm{H} 12$ singlet $\left(\delta_{\mathrm{H}} 4.19\right)$ showed $\mathrm{HMBC}$ correlations to $\mathrm{C} 2$ and $\mathrm{C} 10\left(\delta_{\mathrm{C}} 108.4,119.4\right)$, and long-range COSY coupling to H10, H14b, and $\mathrm{H} 16\left(\delta_{\mathrm{H}} 7.08,2.73,2.41\right)$. H12 and $\mathrm{H} 16$ HMBC correlate to the $\mathrm{C} 15$ ketone carbonyl $\left(\delta_{\mathrm{C}} 205.7\right)$ indicative of an acetyl group at C12. The $\mathrm{C} 17$ methyl mutually correlates with $\mathrm{H} 12$, and $\mathrm{H}_{3} 17$ singlet correlates to $\mathrm{C} 13$ and $\mathrm{C} 14\left(\delta_{\mathrm{C}} 71.9,47.7\right)$. The $\mathrm{C} 13$ chemical shift $\left(\delta_{\mathrm{C}} 71.9\right)$ indicates deshielding consistent with a hydroxyl substituent. HMBC correlations from $\mathrm{C} 14$ protons to $\mathrm{C} 1$ and $\mathrm{C} 2\left(\delta_{\mathrm{C}} 202.5\right.$, 108.4) complete the ring, with $\mathrm{C} 1$ resonance indicative of a ketone.

This compound structure is a novel chemical entity, with no exact matches in any searched database. A substructure search of the SciFinder database however, revealed that the compound 1 structure matched the heavier half of the antibiotic setomimycin, produced by Streptomyces aurantiacus ${ }^{174,175}$. 

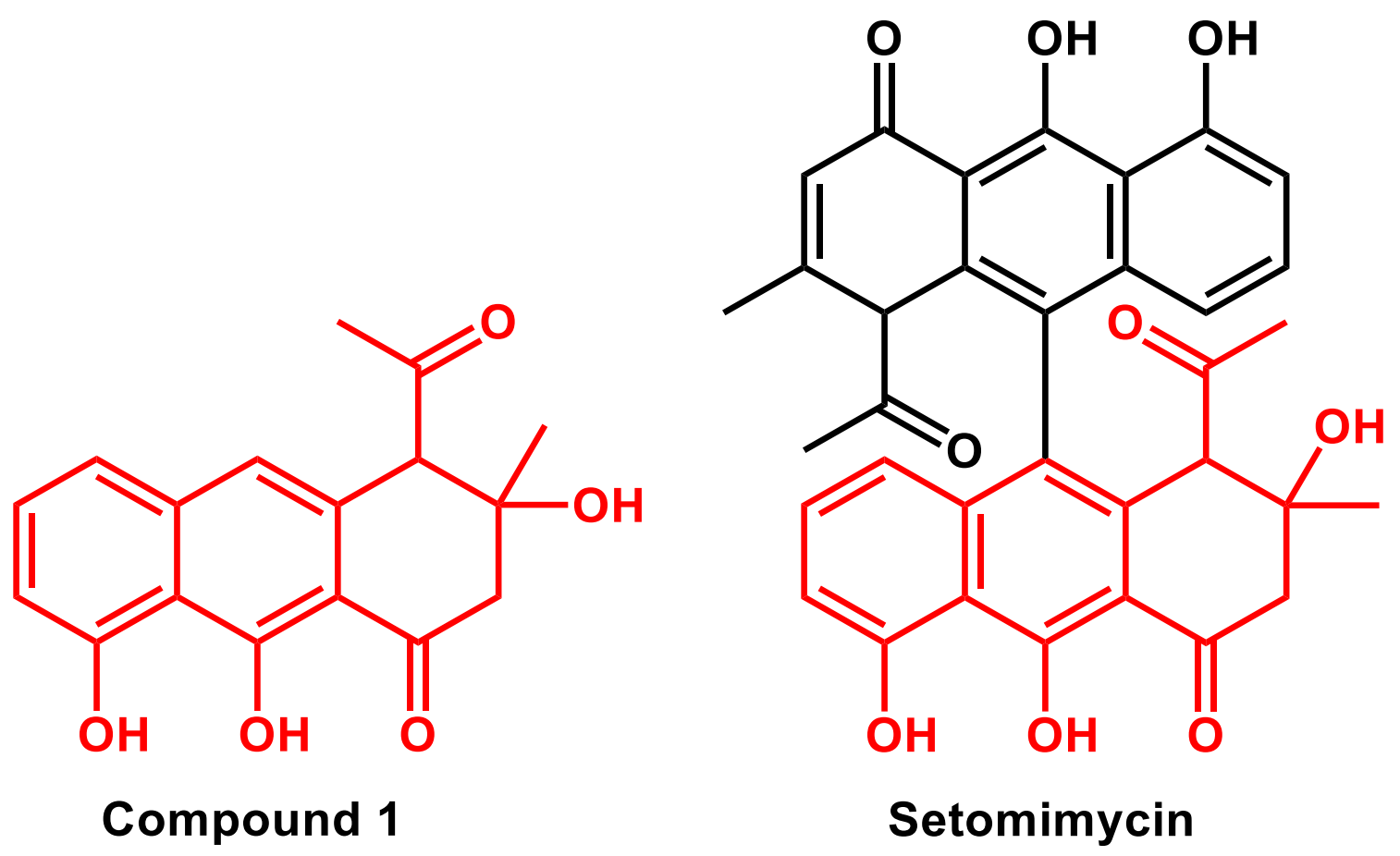

Figure 5.4 - Comparison of chemical structures of compound 1 and setomimycin. The identical regions have been coloured red for emphasis. Note setomimycin is a heterodimer, where one of the non-aromatic hydroxyls has seemingly been removed by dehydration.

Setomimycin is thought to be produced as two polyketide precursors that are dimerised in biosynthesis ${ }^{176}$. It is unclear whether it is formed as a heterodimer, or if the dehydration of one half of the molecule occurs post dimerization. The precursor setomimycin monomer has not been isolated ${ }^{175,176}$.

\subsection{Biosynthetic gene cluster annotation}

Resequencing of the cosmid by Illumina HiSeq 150 bp PE permitted full assembly and resolution of the cluster DNA sequence. The assembly of this sequencing data was similar in process to the assembly of the cosmid pools described in Chapter 4 . In brief, the raw Illumina reads were pre-processed to remove pWEB::tnc vector sequence and contaminating E. coli gDNA sequences (using bowtie2 ${ }^{132}$ ), followed by read quality trimming and sequencing adapter removal by Trimmomatic ${ }^{133}$. The resulting processed Illumina PE reads were then assembled using SPAdes genome assembler ${ }^{129}$. The resulting 
contig sequence was confirmed by mapping Sanger sequencing data from the isolated cosmid on the assembly. This single verified contig of length 36429 bp was then analysed by antiSMASH to detect ORFs and key biosynthesis genes ${ }^{22}$. antiSMASH determined that the contig contained a type II PKS gene cluster that was predicted to produce an angucycline product class compound.

I then further analysed the detected ORFs prior to biosynthetic gene cluster annotation. The antiSMASH-derived GenBank file was downloaded, and the translated amino acid sequences for each ORF collected from the file using a python script (gbk_to_faa.py, Appendix A). These amino acid sequences were then examined by BLASTp against two reference databases; the MIBiG database (v1.4) ${ }^{21}$ as a set of verified natural product biosynthesis genes, and the National Center for Biotechnology Information non-redundant protein sequences database (NCBI-nr). A combination of the antiSMASH and BLAST results (including conserved domain database search - CDD) were used to assign putative function to all full genes in the gene cluster (summarised in Figure 5.5 and Table 5.3).

As the structure of compound $\mathbf{1}$ bears resemblance to one-half of the setomimycin structure (Figure 5.4), I was interested whether the setomimycin biosynthetic pathway was similar to the biosynthetic gene cluster studied here. To evaluate this, I downloaded the genome sequence of the setomimycin producer strain (Streptomyces aurantiacus JA 4570, GenBank accession AOPZ00000000.1) and analysed the genome assembly with antiSMASH ${ }^{22}$. This detected 49 biosynthetic gene clusters, only one of which was a type II PKS cluster. The amino acid sequences from these genes were then aligned to the D9 metagenome cluster sequences using BLASTp as above. 


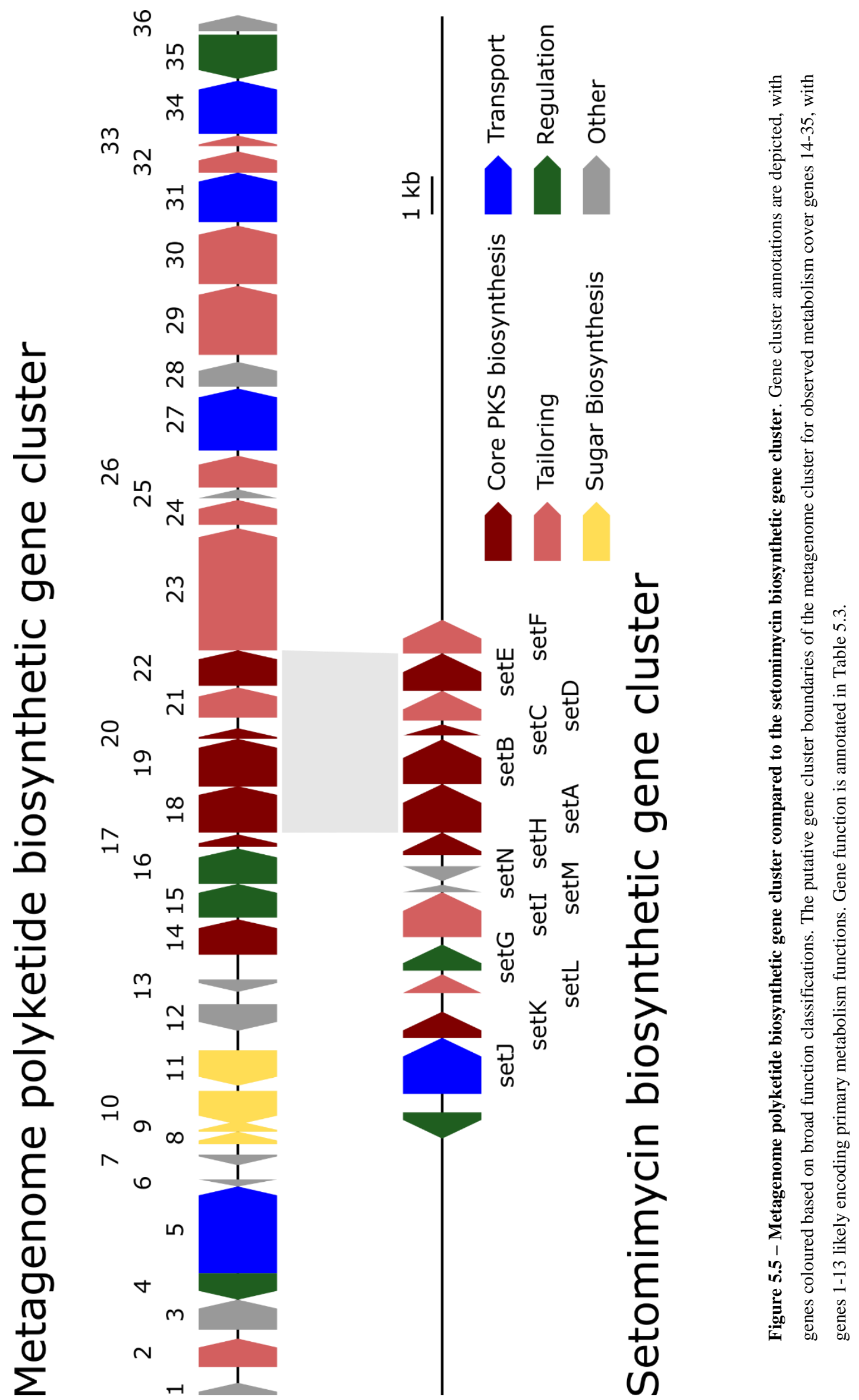




\begin{tabular}{|c|c|c|c|c|}
\hline Gene & Assigned function & $\begin{array}{l}\text { Setomimycin } \\
\text { homologue }\end{array}$ & Function & $\begin{array}{l}\% \text { aa } \\
\text { identity }\end{array}$ \\
\hline \multicolumn{5}{|l|}{1} \\
\hline 2 & Oxidoreductase & & & \\
\hline 3 & Hypothetical protein & & & \\
\hline 4 & Regulatory protein & & & \\
\hline 5 & MMPL family transporter & & & \\
\hline 6 & Hypothetical protein & & & \\
\hline 7 & Hypothetical protein & & & \\
\hline 8 & beta-galactosidase & & & \\
\hline 9 & beta-galactosidase & & & \\
\hline 10 & Fructose-biphosphate aldolase & & & \\
\hline 11 & Sugar isomerase & & & \\
\hline 12 & Hypothetical protein & & & \\
\hline 13 & Hypothetical protein & & & \\
\hline 14 & Acetyltransferase (AT) & & & \\
\hline 15 & Response regulator & SetG & Regulator & 64.574 \\
\hline 16 & Regulatory protein & & & \\
\hline 17 & Cyclase $(\mathbf{C} 4-\mathbf{C 1 7})$ & SetH & Cyclase & 64.545 \\
\hline 18 & Ketosynthase alpha $\left(\mathbf{K S}_{\alpha}\right)$ & SetA & $\mathbf{K S}_{\boldsymbol{\alpha}}$ & 78.537 \\
\hline 19 & Ketosynthase beta $\left(\mathrm{KS}_{\beta}\right)$ & SetB & $\mathbf{K} \mathbf{S}_{\beta}$ & 70.277 \\
\hline 20 & Acyl carrier protein (ACP) & SetC & $\mathbf{A C P}$ & 60.241 \\
\hline 21 & Ketoreductase (C9) & SetD & Ketoreductase & 76.628 \\
\hline 22 & Cyclase (C7-C12)/ dehydrase & SetE & Cyclase & 61.736 \\
\hline 23 & Hydroxylase/reductase & & & \\
\hline 24 & Oxidoreductase & & & \\
\hline 25 & Hypothetical protein & & & \\
\hline 26 & Hydrolase (dienelactone hydrolase) & & & \\
\hline 27 & Drug resistance transporter & & & \\
\hline 28 & Cation binding domain protein & & & \\
\hline 29 & Oxidoreductase & & & \\
\hline 30 & Dehydrogenase & & & \\
\hline 31 & Proton exchange transport & & & \\
\hline 32 & Oxidoreductase & & & \\
\hline 33 & AB monooxygenase & & & \\
\hline 34 & Proton exchange transport & & & \\
\hline 35 & Sigma factor & & & \\
\hline
\end{tabular}

$\begin{array}{ll}\text { SetJ } & \text { Resistance transporter } \\ \text { SetK } & \text { Cyclase } \\ \text { SetL } & \text { Unknown } \\ \text { SetI } & \text { Cytochrome P450 } \\ \text { SetM } & \text { Unknown } \\ \text { SetN } & \text { Hypothetical protein } \\ \text { SetF } & \text { Hydrolase/thioesterase }\end{array}$

Table 5.3 - Metagenome polyketide biosynthetic gene cluster gene annotations and alignment of homologous proteins from the setomimycin biosynthetic gene cluster. Metagenome genes 14-35, which comprise the putative biosynthetic gene cluster, have been made bold for emphasis. 
The metagenome cosmid contains 34 open reading frames (2-35), with genes 1 and 36 truncated by the cosmid boundaries. The biosynthetic gene cluster appears encoded as a single expression unit, with a unidirectional arrangement of genes 14-34 that is capped by an RNA polymerase sigma factor gene (35). The products of genes 18, 19 and 20 are predicted to form the central type II PKS system of $\mathrm{KS}_{\alpha}, \mathrm{KS}_{\beta}$, and $\mathrm{ACP}$, with subsequent cyclase modifications to the polyketide product by the products of genes 17 and 22 . A lone acyltransferase encoded by gene 14 may play a role in the core compound biosynthesis, or polyketide starter unit loading ${ }^{42,177}$. Reductase $(21,23,24,29$, and 32), hydrolase (26), and dehydrogenase (30) gene products likely play key roles in the biosynthesis. The three transport related genes $(27,31$, and 34$)$ in the core expression unit are likely involved in metabolite efflux, which may explain the ready recovery of compounds $\mathbf{1}$ and $\mathbf{2}$ from the clarified expression culture medium. Regulatory genes may have a role in controlling expression of the gene cluster, or specific genes, in response to specific stimuli $(15,16$, and $35)$.

Using the type II PKS module available on antiSMASH 5.0, more detailed gene product functions could be predicted ${ }^{178}$. This module predicted an acetyl-CoA starter unit, that the $\mathrm{KS}_{\beta}$ was selective for 8 or 9 malonyl extension units, and that cyclase 17 was specific for C4-C17 cyclisation (or C2-C19), cyclase 22 was specific for $\mathrm{C} 7-\mathrm{C} 12$ cyclisation and ketoreductase 21 reduces C9. Using these insights, a proposed biosynthesis scheme was devised (Figure 5.6). In this postulated scheme, a nonaketide precursor is synthesised by core type II PKS enzymes from 1 acetyl-CoA and 8 malonyl-CoA monomers. The initial ring is then cyclised and aromatised by the function of the C9-reductase and the C7-C12 di-domain cyclase, by established mechanisms ${ }^{179}$. The tricyclic structure is then formed by reactions catalysed by reductase enzymes, and the $\mathrm{C} 4-\mathrm{C} 17$ cyclase. Decarboxylation to liberate $\mathrm{C} 1$ would produce compound $\mathbf{1}$, which may be catalysed by hydrolase or other enzymes. As the recovered gene cluster is likely only a partial pathway, and as only one of the metabolite products has been recovered, this scheme is speculative and likely incomplete. 


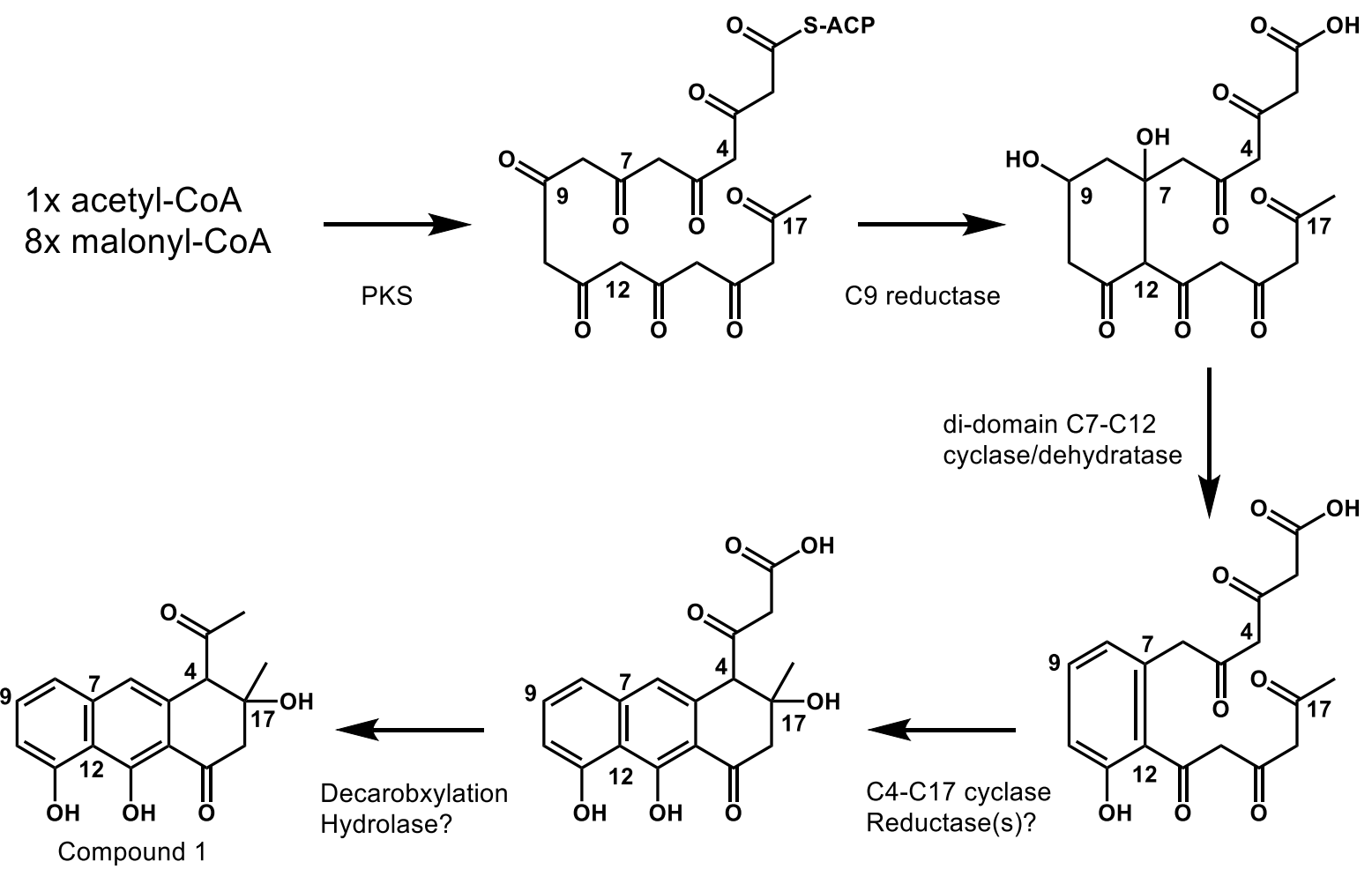

Figure 5.6 - Possible biosynthesis scheme for compound 1. The proposed scheme was devised based on type II PKS enzyme substrate specificities predicted by antiSMASH 5.0.

The only setomimycin genes that aligned to genes in the D9 metagenome cosmid insert sequence were within the putative cluster boundaries (genes 14-35). These seven genes consisted of the set $G$ regulator, and a cassette of genes with identical arrangement to the metagenome cluster: setA $\left(\mathrm{KS}_{\alpha}\right)$, setB $\left(\mathrm{KS}_{\beta}\right)$, setC $(\mathrm{ACP})$, setH and setE (cyclases), and setD (ketoreductase). However, orthologues of these seven genes are core to the majority of type II polyketide biosynthesis pathways, and often occur in these arrangements, therefore it is not possible to conclude a relatedness between the two gene clusters. The relatively low alignment identities (mean $68.1 \%$ identity) between the individual amino acid sequences suggests substantial divergence has occurred between them. Other genes in setomimycin biosynthesis did not contain homologues in the metagenome sequence: setJ (transport), setK (cyclase), setL (unknown), setM (putative ferredoxin), set $N$ (unknown), setF (hydrolase) and most importantly setI (cytochrome P450). In most cases (setJ, setK, $s e t F)$ the metagenome cluster has other genes with similar annotated function, however there is no gene that could complement SetI function. SetI homologues have been implicated in the dimerization of polyketide monomers in compounds similar to setomimycin ${ }^{176}$. 
A cassette of four genes involved in sugar metabolism may indicate a glycosylated product $\left(8,9,10\right.$, and 11), which is common in angucycline class compounds ${ }^{43}$. Genes 8 and 9 contain conserved domains for the $\mathrm{C}$-terminal and trimerisation domains $\beta$-galactosidase respectively. However, no glycosyltransferase genes are present that would catalyse the addition of a sugar to the core compound. These genes are also outside of the apparent biosynthetic transcriptional unit, and therefore may not be associated with this biosynthetic gene cluster. The genes also do not appear to be involved in the biosynthetic pathways for deoxy sugars commonly associated by glycosylated secondary metabolites ${ }^{46,47}$, and therefore may be associated with primary metabolism instead.

\subsection{Bioactivity testing}

Initial investigations into the bioactivity of metabolites from the D9 cosmid biosynthesis pathway had indicated some Gram-positive antibacterial activity with HPLC peak 2 metabolites (section 5.3). To investigate the potential bioactivities of the purified compound 1 sample, a panel of test strains were analysed for potential antibacterial, antifungal, or antitumor bioactivity. Minimum inhibitory concentration (MIC) analysis was performed against a panel of microbes: $E$. coli 7NT (Gram-negative bacterium with efflux pump gene tolC knocked out), B. subtilis 168 (Gram-positive bacterium), S. cerevisiae $\triangle \mathrm{PDR}$ (yeast with pleotropic drug resistance knockout). Positive control compounds included tetracycline (for E. coli and B. subtilis), nystatin (for S. cerevisiae) and mithramycin A (for all). Media for culture growth and MIC experiments were MH broth (E. coli and B. subtilis) and YPD (S. cerevisiae). In each case three biological replicates were performed over three consecutive days. In parallel with the MIC experiments, Dr Abigail Sharrock performed cytotoxicity assays of compound $\mathbf{1}$ on human colon carcinoma (HCT-116) cells, to investigate potential antitumor activity.

Compound $\mathbf{1}$ has weak antibacterial activity, although it was slightly more potent against the Gram-negative E. coli 7NT test strain than B. subtilis. This anti Gram-negative activity may be amplified by the deletion of tolC in the E. coli test strain, reducing Gram-negative drug-efflux capability. Compound 1 inhibited $S$. cerevisiae growth only at very high concentrations, and did not exhibit cancer cell cytotoxicity. 


\begin{tabular}{|c|c|c|c|c|c|c|}
\hline & \multicolumn{2}{|c|}{ Compound 1} & Tetracycline & Nystatin & \multicolumn{2}{|c|}{ Mithramycin A } \\
\hline Test strain & $\begin{array}{l}\text { MIC } \\
(\mu \mathrm{g} / \mathrm{mL})\end{array}$ & $\begin{array}{l}\mathrm{IC}_{50} \\
(\mu \mathrm{M})\end{array}$ & $\begin{array}{l}\text { MIC } \\
(\mu \mathrm{g} / \mathrm{mL})\end{array}$ & $\begin{array}{l}\text { MIC } \\
(\mu \mathrm{g} / \mathrm{mL})\end{array}$ & $\begin{array}{l}\text { MIC } \\
(\mu \mathrm{g} / \mathrm{mL})\end{array}$ & $\begin{array}{l}\mathrm{IC}_{50} \\
(\mu \mathrm{M})\end{array}$ \\
\hline E. coli 7NT & 32 & & 0.125 & & $>32$ & \\
\hline B. subtilis 168 & 64 & & 1 & & 0.004 & \\
\hline S. cerevisiae $\triangle \mathrm{PDR}$ & 128 & & & 0.5 & 32 & \\
\hline HCT-116 & & $>200$ & & & & 0.017 \\
\hline
\end{tabular}

Table 5.4 - Bioactivity assay results of antimicrobial and cytotoxicity assays. Antimicrobial minimum inhibitory concentration (MIC) assays were conducted for compound $\mathbf{1}$ with positive controls of tetracycline (antibacterial), nystatin (antifungal) and mithramycin A (Gram-positive antibacterial). Tumour cell cytotoxicity was performed with mithramycin A as a positive control agent. The data in the table were determined across at least three independent experiments.

This toxicity profile is markedly different to the reported figures for setomimycin, which exhibited Gram-positive MIC from 1.56-25 $\mu \mathrm{g} / \mathrm{mL}$, Gram-negative and fungal MIC >100 $\mu \mathrm{g} / \mathrm{mL}$, and inhibition of tumour growth in a mouse model ${ }^{174}$.

\subsection{Discussion}

In this chapter, a cosmid containing a type II PKS biosynthetic pathway was recovered from the Half Moon Bay metagenome library, and its gene content and metabolic output were studied. Heterologous expression of this pathway in $S$. albus resulted in the production of three pathway associated metabolites (compounds 1-3), however only one (compound 1) was stable, soluble, and produced in sufficient quantities for structure elucidation.

Compounds $\mathbf{1}$ and $\mathbf{2}$ were initially isolated as a mixture (eluting as a single peak in initial reversed-phase HPLC purification trials), which possessed Gram-positive bioactivity beyond that observed when compound 1 was eventually purified. It is possible this bioactivity was associated with compound 2 , however instability made characterising compound $\mathbf{2}$ intractable. It is also possible that compound $\mathbf{2}$ is a "pathway intermediate" compound, and that a complete biosynthetic pathway would quickly modify the unstable metabolite to a more stable form. Following this reasoning, it is unclear whether compound $\mathbf{1}$ or compound $\mathbf{2}$ represents a more "complete" metabolite along the metabolic pathway, or if the compounds exist as degradation/shunt products of the biosynthesis. 
The chemical structure of compound $\mathbf{1}$ is analogous to one half of the heterodimer antibiotic setomimycin. Setomimycin is thought to be biosynthesised as monomers, which are dimerised by SetI (cytochrome P450) into the active antibiotic. The biosynthetic gene cluster on cosmid D9 did not contain a SetI homologue, and no mass associated with dimerised compound $\mathbf{1}$ or setomimycin was observed in LC-MS analysis of the S. albus D9 fermentation extract. It may be that the biosynthetic gene cluster on cosmid D9 is incomplete, and if complete may include a setI homologue to produce an enzyme to catalyse a dimerization and produce a compound similar or identical to setomimycin. Alternatively, other tailoring functions may occur, such as glycosylation to increase metabolite stability or specificity/potency of bioactivity ${ }^{42,46}$.

The isolated compound 1 possessed weak Gram-positive and Gram-negative antibacterial activity, without inhibition of fungal or cancer cell growth. This is notably different to the previously characterised compound setomimycin, which possessed strong Gram-positive and cancer cell growth inhibition, but no Gram-negative activity. The bioactivity profile of compound 1 presents as a weak-moderately active general antibacterial toxin, whereas setomimycin has more potent and specific toxicity profile. This may indicate the SetI catalysed dimerization functions to activate setomimycin for a specific role or challenge response (e.g. Gram-positive bacterial competition).

Compound 1 represents a novel chemical structure that has not been previously observed or characterised. Similar structures have been implicated in the biosynthesis of not only setomimycin, but also other aromatic polyketide pathways including the julichromes and spectomycin 175,176,180,181. The compound 1 structure satisfies Lipinski's "rule of five" for drug-likeness without violation, with only 3 hydrogen bond donors, 5 acceptors, a low molecular weight of $300.31 \mathrm{~g} / \mathrm{mol}$, and a calculated $\operatorname{logP}$ of $0.48^{182}$. Compound 1 has general moderate-weak bioactivity against a range of targets, and has potential as a "leadlike" compound for medicinal chemistry modification. Future studies should focus on recovery of the remainder of the genes in this biosynthetic gene cluster from the Half Moon Bay library to complete the biosynthetic pathway and characterise additional compounds (these strategies are discussed in detail in section 7.3.2). 


\subsection{Discovery and reconstitution of novel aureolic acid biosynthesis gene cluster and characterisation of major metabolites}

\subsection{Summary}

This chapter describes how a biosynthetic gene cluster predicted to encode for an aureolic acid biosynthesis pathway was reconstructed from overlapping cosmids recovered from the Half Moon Bay cosmid metagenome library. PCR screening was used to recover a set of overlapping cosmid clones, and the genetic pathway was reconstituted using yeast transformation associated recombination to reform the entire $43 \mathrm{~kb}$ genetic pathway as a single contiguous Bacterial Artificial Chromosome (BAC).

Heterologous expression of this complete biosynthetic gene cluster in $S$. albus resulted in the production of a number of pathway specific metabolites, including at least one novel compound with potent bioactivity. The major metabolite of this biosynthetic pathway was isolated (compound 4), and structurally characterised by MS and NMR analysis. Compound 5 was identified by bioactivity-guided fractionation, and characterised by analysis of MS/MS fragmentation. Key features of the biosynthetic gene cluster also support our proposed structure for compound $\mathbf{5}$, which we propose is the product of a single oxidative ring opening enzyme acting on compound 4. Bioactivity studies of the major metabolite compound $\mathbf{4}$ indicated moderate Gram-positive antibacterial activity and anticancer cytotoxicity.<smiles></smiles>

Compound 4<smiles>COC(C(=O)C(O)C(C)O)C1Cc2cc3cc(OC4CC(C)(O)C(O)C(C)O4)c(C)c(O)c3c(O)c2C(=O)C2OC3CC(OC4CC(OC5CC(C)(O)C(O)C(C)O5)C(O)C(C)O4)C(C)OC3C1O2</smiles>

Compound 5 


\subsection{Introduction}

As briefly described in Chapter $5, \mathrm{KS}_{\alpha}$ degenerate PCR screening of the HM cosmid metagenome library resulted in the recovery of a cosmid containing a partial biosynthetic gene cluster with cluster level homology to the two known aureolic acid biosynthetic gene clusters - mithramycin from Streptomyces argillaceus ${ }^{49,183}$, and chromomycin $\mathrm{A}_{3}$ of Streptomyces griseus subsp. griseus ${ }^{48}$. Although mithramycin and chromomycin $\mathrm{A}_{3}$ are the only characterised aureolic acid biosynthetic pathways, multiple other members of the compound family have been characterised ${ }^{49}$, including the olivomycins produced by Streptoverticillum cinnamoneum ${ }^{184}$, durhamycin A from Actinoplanes durhamensis ${ }^{185}$, UCH9 by a Streptomyces sp. ${ }^{186}$, and chromocyclomycin from Streptomyces LA-7017 ${ }^{187}$. The aureolic acid structures are characterised by a tricyclic polyketide that contains an aliphatic sidechain, and two oligosaccharide chains composed of 2,6-dideoxysugars (Figure 6.1). The exception is chromocyclomycin, which has a tetracyclic aglycone. Structural differences between compounds in this family relate to the number and type of deoxy sugars in the oligosaccharide chains (combinations of D-olivose, D-oliose, Dmycarose, and L-chromose $\mathrm{B}$, with derivatives via O-methylation and O-acetylation), the glycosidic linkages to the deoxy sugars $(\alpha / \beta)$, and a small alkyl chain (either methyl, isobutyl, or absent in olivomycin A). 


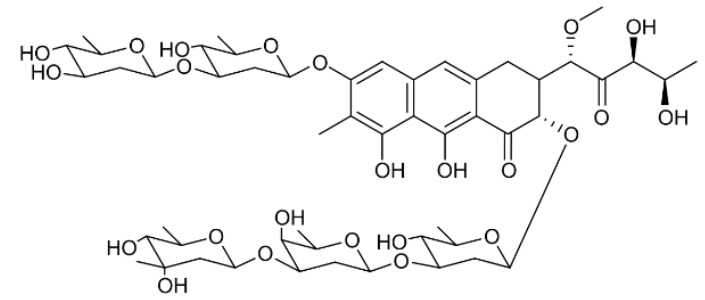

Mithramycin

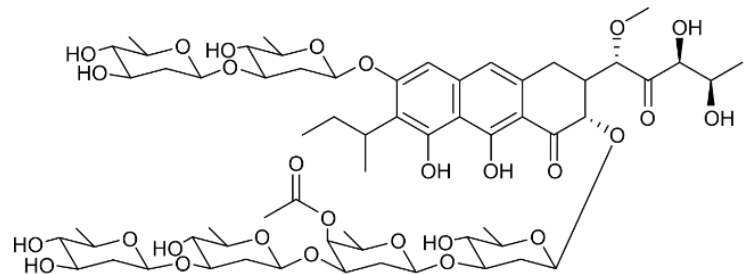

Durhamycin A

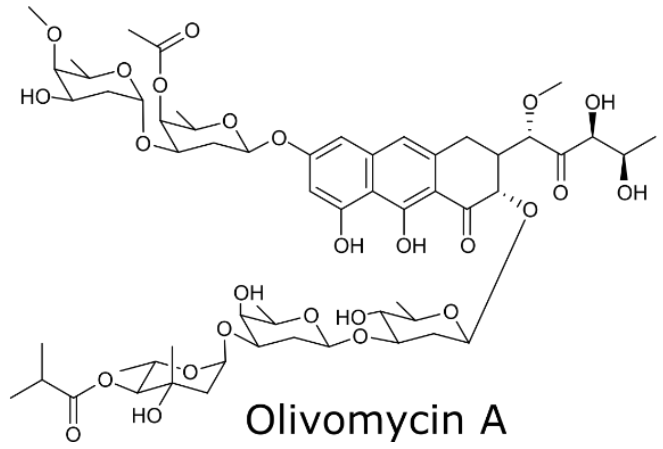

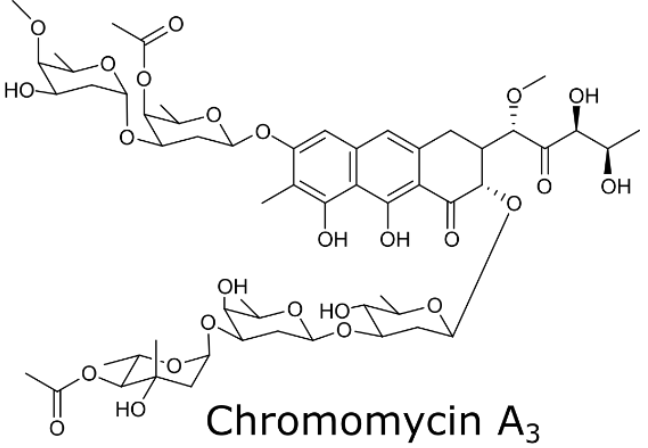
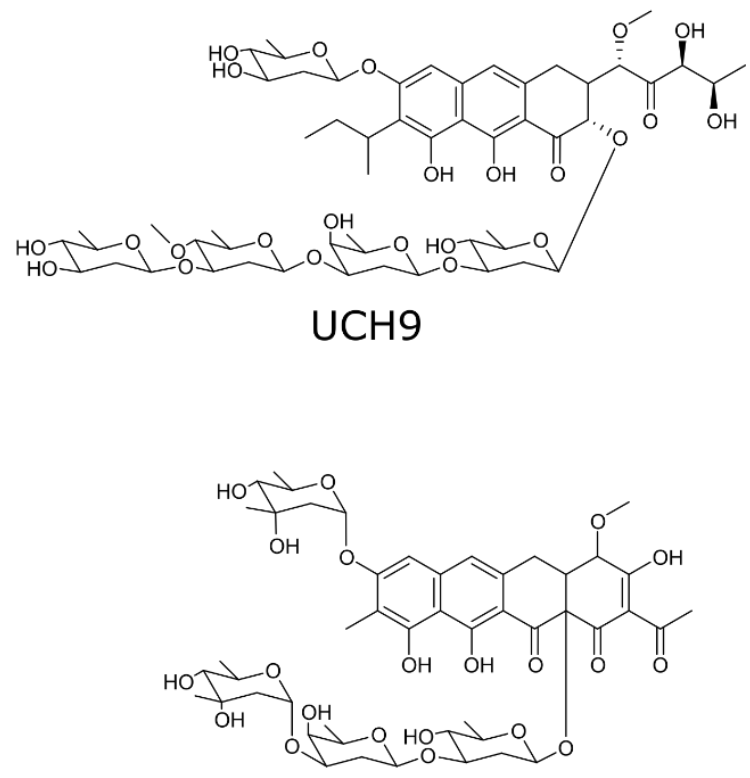

Chromocyclomycin

Figure 6.1 - Chemical structures of the aureolic acid family of compounds.

The aureolic acid family of compounds has received substantial attention from the scientific community, as evidenced by the growth in publications referencing aureolic acid, mithramycin, and/or chromomycin (Figure 6.2). The aureolic acids are potent Grampositive antibiotics ${ }^{49}$, with low $\mu \mathrm{g} / \mathrm{mL}$ MIC against VRE and sub $\mu \mathrm{g} / \mathrm{mL}$ MIC values against MRSA ${ }^{188}$. However, most interest in these compounds and their derivatives is a result of their potent anticancer activity, with $\mathrm{IC}_{50}$ values in the low $\mathrm{nM}$ range ${ }^{189}$. There is a desire to find congeners or methods of administration that can retain this desired effect while mitigating their significant toxicity profile ${ }^{170,171}$. The aureolic acids have been the subject of a number of reviews and perspectives, covering not only their biosynthesis ${ }^{49}$ and anticancer method of action ${ }^{170}$, but also the search for congeners/analogues of mithramycin like compounds - "mithralogs" ${ }^{171}$. Such compounds have been created through chemical modification of the compounds structure ${ }^{190-193}$, in the course of evaluating biosynthesis by 
pathway gene knockout ${ }^{48,189,194}$, and by way of combinatorial biosynthesis, i.e. inclusion of promiscuous tailoring genes from other polyketide pathways ${ }^{51,195-197}$.

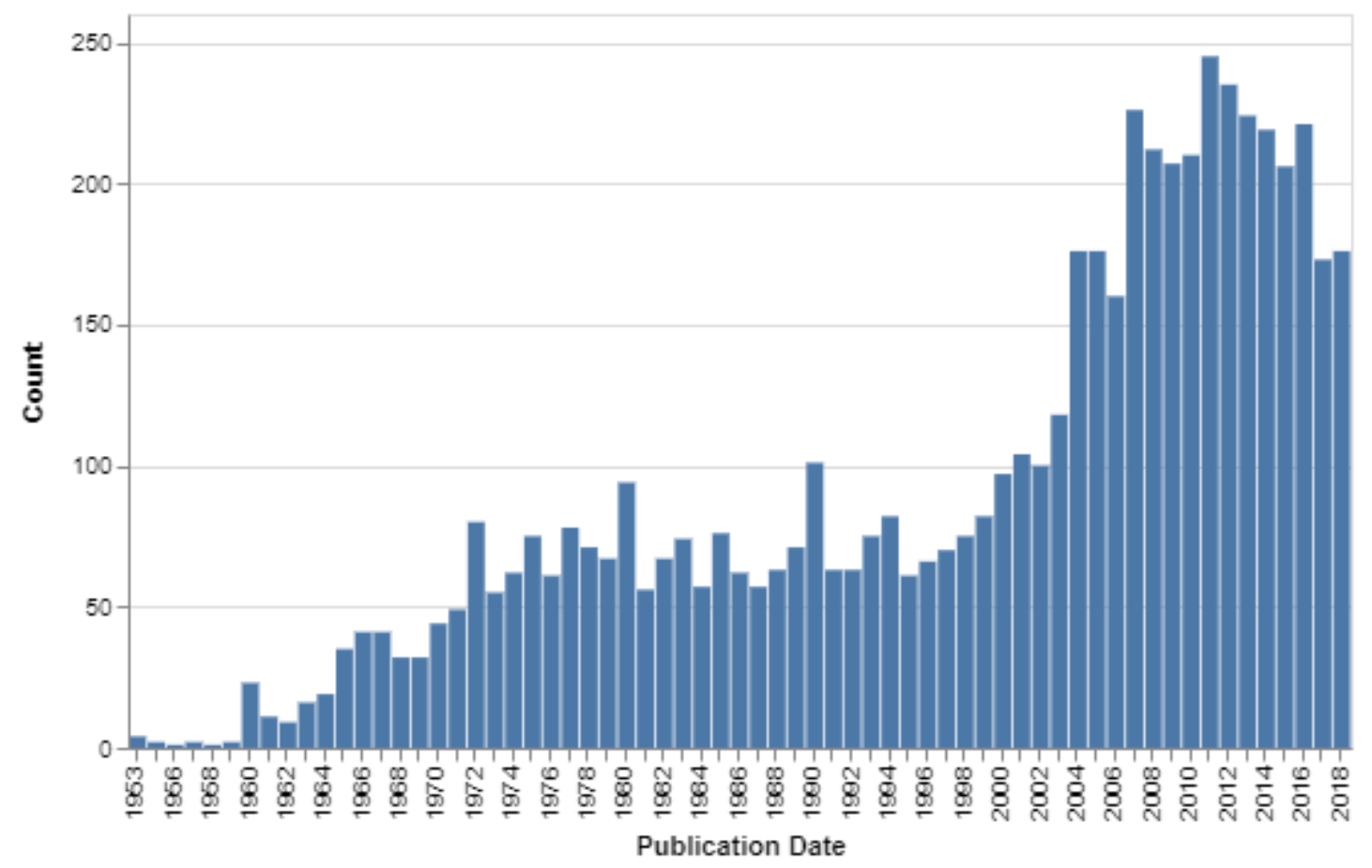

Figure 6.2 - Number of publications in SciFinder Scholar that make reference to aureolic acid, mithramycin or chromomycin by year up to 2018.

The complete biosynthetic pathways of mithramycin and chromomycin $\mathrm{A}_{3}$ have been characterised, with gene assignments validated by gene knockout studies, and isolation of many pathway intermediates ${ }^{49}$. In general, the two compounds follow very similar biosynthetic schemes, which are likely common to all known aureolic acids. 

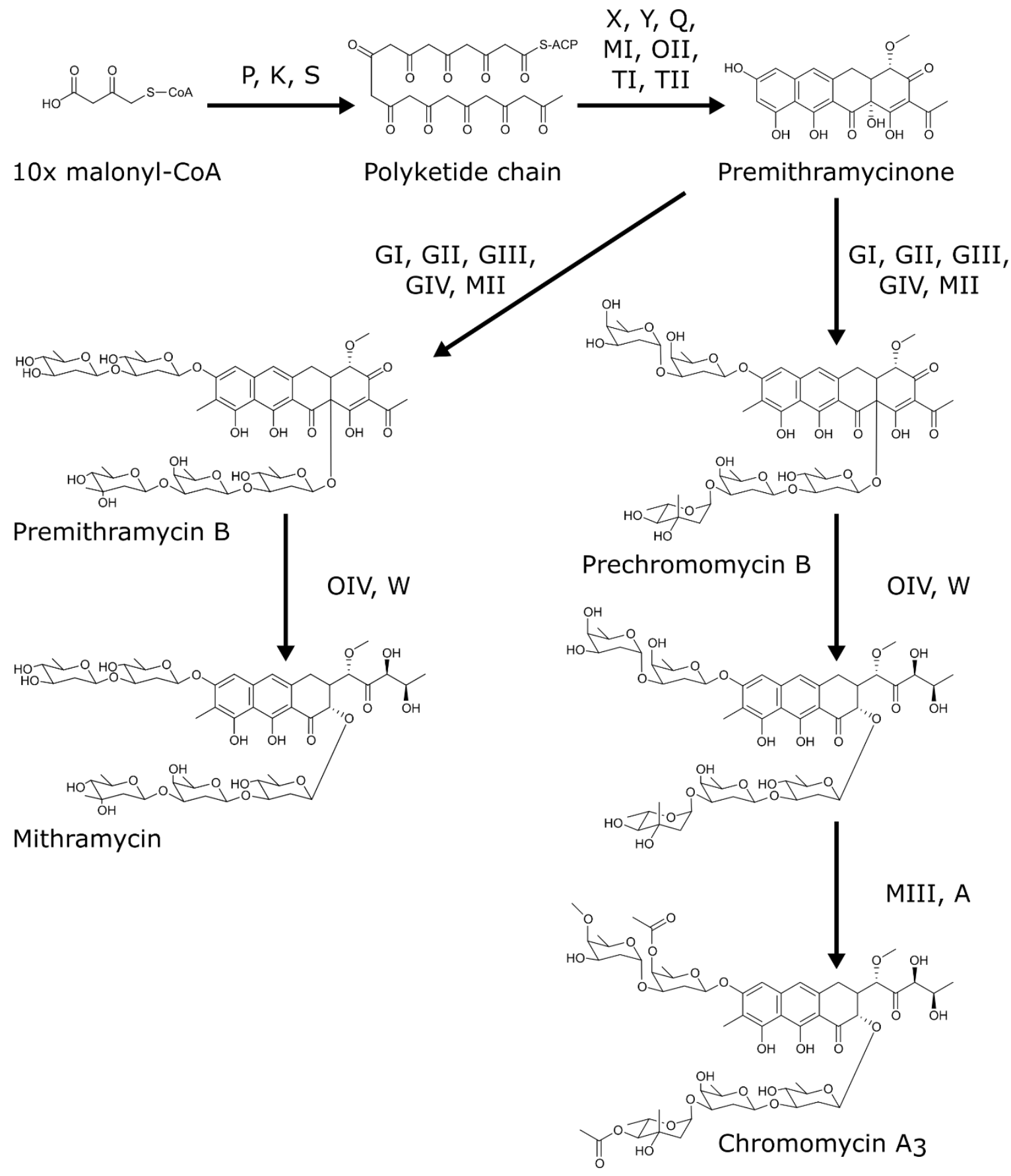

Figure 6.3 - Condensed biosynthesis scheme for aureolic acids mithramycin and chromomycin $\mathbf{A}_{3}$.

Type II polyketide biosynthesis initiates the pathway (Figure 6.3), with iterative condensation of 10 malonyl-CoA derived malonate residues into a decaketide chain (genes $\mathrm{P}, \mathrm{K}, \mathrm{S}$ encoding the $\mathrm{KS}_{\alpha} \mathrm{KS}_{\beta}$ and ACP respectively). The chain is then cyclised to form the initial tetracyclic core, by a combination of cyclisation events that are either spontaneous, or catalysed by cyclase $(X, Y)$, or aromatase $(\mathrm{Q})$ enzymes. Methyltransferase (MI), oxygenase (OII) and ketoreductase (TI, TII) enzymes modify this core to produce the 
primary aglycone, premithramycinone. The pathways for mithramycin and chromomycin $\mathrm{A}_{3}$ then diverge, as the respective glycosyltransferase enzymes (GI-GIV) catalyse addition of different deoxy sugars to the aglycone scaffold. Each gene cluster also contains a set of genes encoding sugar biosynthesis to produce the requisite deoxy sugars from a primary metabolism derived D-glucose-1-phosphate starting unit. Methyltransferase (MII) catalysed methylation also occurs in both pathways, to produce the biologically inactive glycosylated tetracyclic compounds, premithramycin B and prechromomycin B. In both pathways, the fourth ring is opened by an oxygenase enzyme (OIV), and the resulting carbon chain reduced by a ketoreductase (W) to produce the aliphatic tail that is required for biological activity of the aureolic acid compounds. In the chromomycin pathway, this compound is then further modified by O-acyltransferase (A) and O-methyltransferase (MIII) enzyme function to produce the final chromomycin $\mathrm{A}_{3}$ structure.

The partial biosynthetic gene cluster on the D3 cosmid was investigated as the basis for this chapter, with the aim characterising a third aureolic acid biosynthesis pathway, and potentially discovering a new metabolite.

\subsection{Reconstitution of the biosynthetic gene cluster}

Initial heterologous expression trials of the D3 cosmid in S. albus indicated low-level production of any pathway specific compounds. In addition to this, analysis of the DNA sequence of the single cosmid revealed a truncated gene with a predicted product that shares high homology with a DNA-repair enzyme critical for self-immunity to aureolic acid toxins (MtrX, CmrX) ${ }^{198}$. The cosmid also lacked homologues of the drug efflux pump genes that are present in the two known aureolic acid biosynthetic gene clusters and contribute to selfimmunity (MtrA, MtrB, CmmA, CmmB) ${ }^{198,199}$. In order to complete the full cluster, the remaining genes needed to be recovered from the HM metagenome library. PCR primers were designed against known sequences of the recovered cosmid (Figure 6.4). By screening successively diluted pools of the metagenome library using the three primer sets, cosmid clones were recovered that contained the same "overlapping" DNA sequences as the original cosmid. By recovering cosmid clones that contained the same DNA sequence as either one of the "edge" primer sets, but not the "middle" primer set, the remainder of the gene cluster sequence was then recovered from the HM cosmid metagenome library (Figure $6.4)$. 

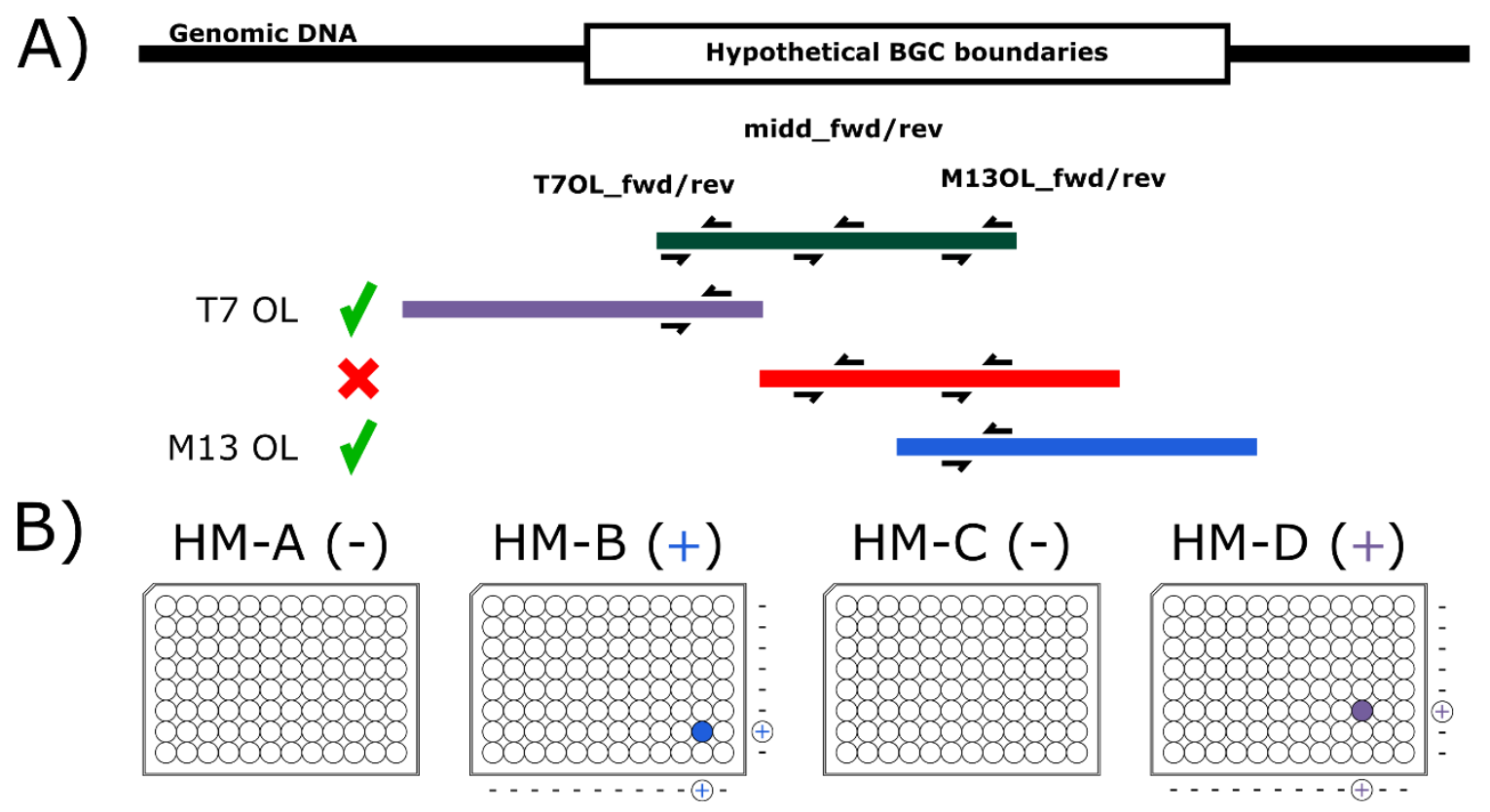

C)

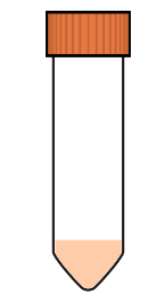

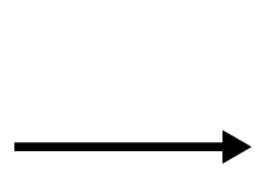

Dilute culture and titre across 384 well plate
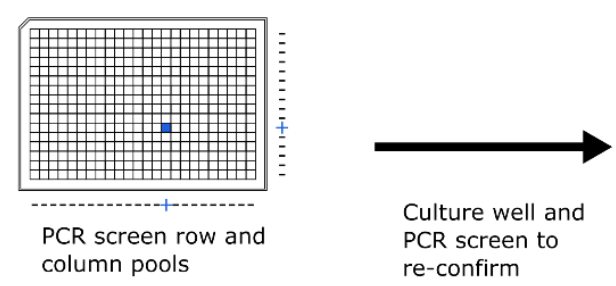

re-confirm
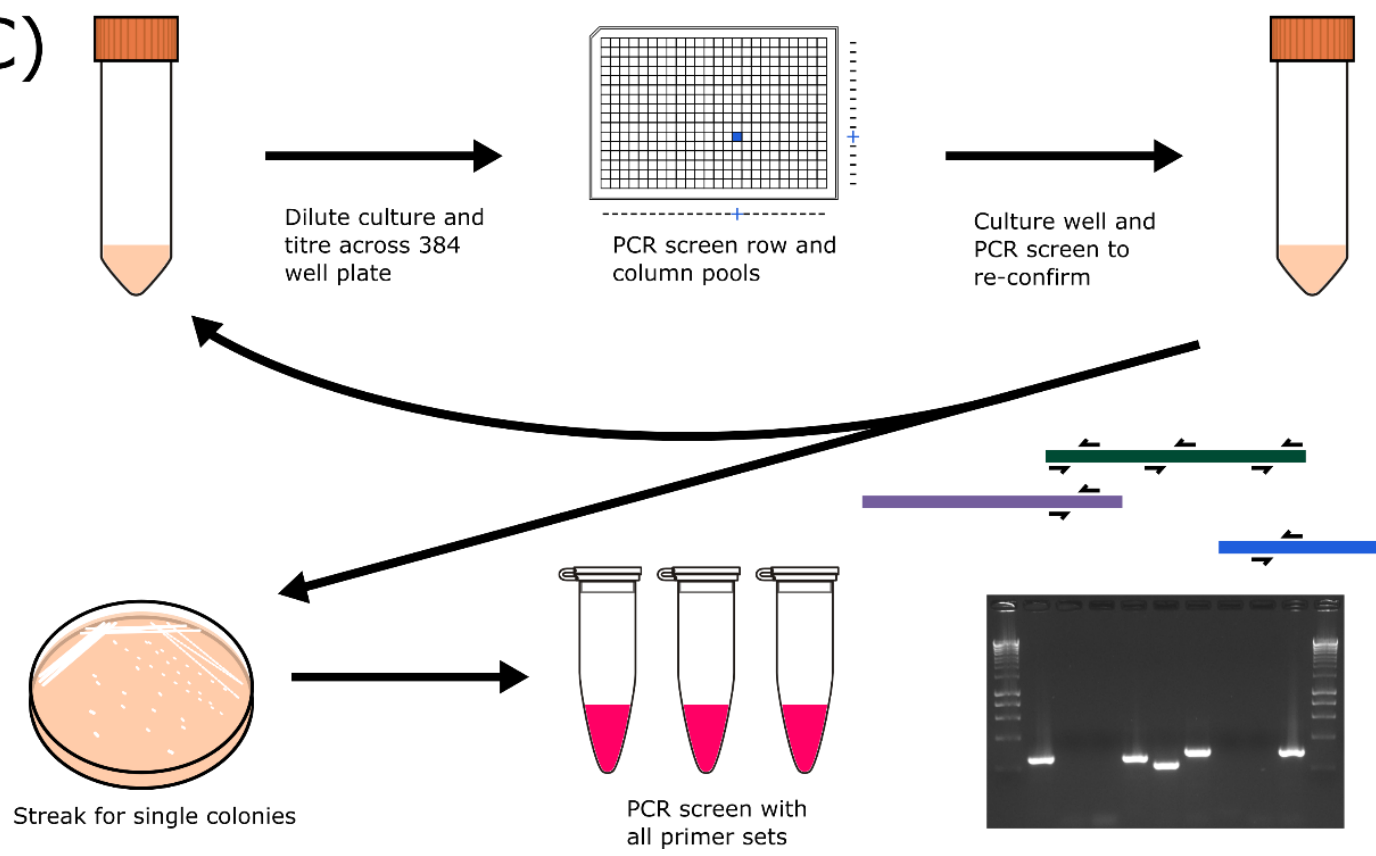

Figure 6.4 - Generic cosmid overlap PCR recovery process. A) An isolated cosmid (green) represents a section of a biosynthetic gene cluster that was originally on a single contiguous section of genomic DNA. Primers are designed from this original cosmid DNA sequence to screen for other cosmids in the metagenome library with matching sequences. By finding cosmids that have the "edge" sequences but not the "middle" sequences, the maximum amount of new information can be recovered (i.e. not the red sequence). B) The library plate row/column pools are screened by PCR, hit wells cultured, and over a series of culture, dilution and PCR screening steps (C) single cosmid sequences can be isolated from the library. Final isolated overlap cosmids PCR results (agarose gel image, bottom right), where the original cosmid (green) amplifies successfully with each primer set, while the recovered overlap cosmids each only hit with a single "edge" primer set. 
Doing this resulted in the successful isolation of cosmid overlaps for each end. The overlap at the T7 end was recovered from HM library plate D, well F10 (23,000 clones), over three rounds; first diluting to around 200 clones per pool, then to five clones per well before picking individual colonies. The overlap at the M13 end was recovered from HM plate B, well G11 (43,000 clones), over three rounds; first diluting to 825 clones per pool, then to seven clones per well before picking individual colonies.

The overlapping cosmids were confirmed by a combination of Sanger sequencing with vector end sequencing primers (T7promoter, M13F-pUC) and the overlap screening primers. The cosmids were then sequenced by Illumina HiSeq (100 bp PE), and assembled using SPADES, with the raw Illumina reads pre-processed to remove pWEB::tnc vector sequence and contaminating E. coli gDNA sequences (using bowtie ${ }^{132}$ ), followed by read quality trimming and sequencing adapter removal by Trimmomatic ${ }^{133}$. The resulting processed Illumina PE reads were then assembled using SPAdes genome assembler ${ }^{129}$. After assembly, the T7OL cosmid insert resolved to a single contig of 33,169 bp, and the M13OL cosmid insert resolved to a contig of $24,030 \mathrm{bp}$, as well as a number of smaller contigs. The M13OL is significantly shorter in length than expected, and examination of the SPADES generated De Bruijn graph revealed this was the result of a long series of repeat sequences that occurred in the cosmid insert outside of the biosynthetic gene cluster. These repeat sequences could not be resolved using the short read sequence data acquired here, however, as the full gene cluster had been sequenced at this point, no further effort was placed into resolving this sequencing artefact.

The three resulting overlapping cosmid sequences were then aligned using Geneious software to give a contiguous $83,941 \mathrm{bp}$ sequence. This was analysed using antiSMASH, revealing that the T7OL cosmid likely did not contain any genes relevant to the aureolic acid biosynthetic gene cluster. The M13OL major contig however, successfully reconstituted the DNA-repair $m t r X$ homologous gene, and contained four genes predicted to be involved in drug efflux, and two regulatory genes. A short gene that was predicted to function as an adenosylhomocysteinase marked the end of the gene cluster before a series of ORFs annotated as transposase genes followed, containing the repeat sequences that broke down the assembly. The assembly recovered thereafter, revealing more transposase genes leading into the end vector sequence of the cosmid. A full description of the genes and architecture of the biosynthetic gene cluster is provided in section 6.4. 

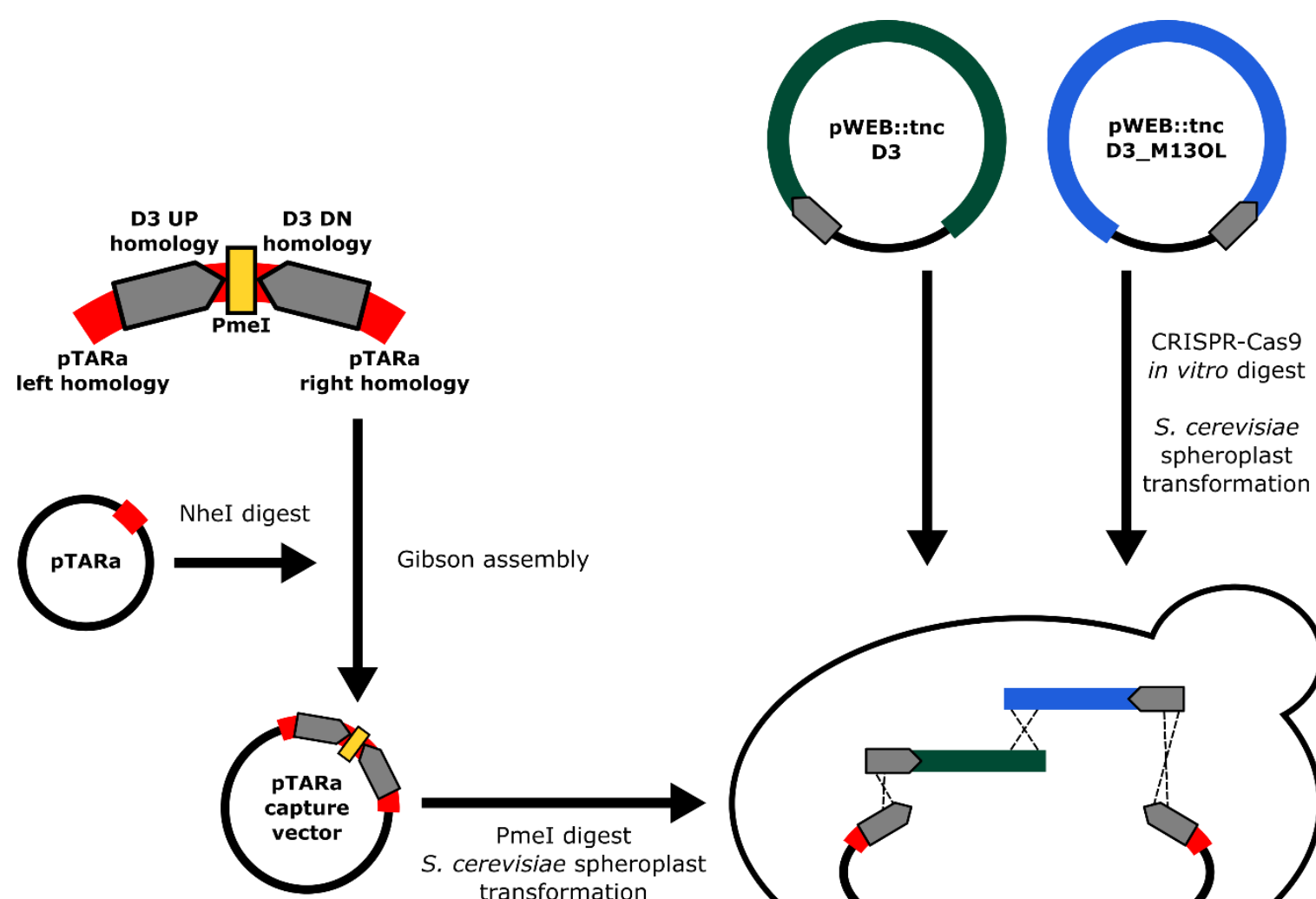

Gibson assembly
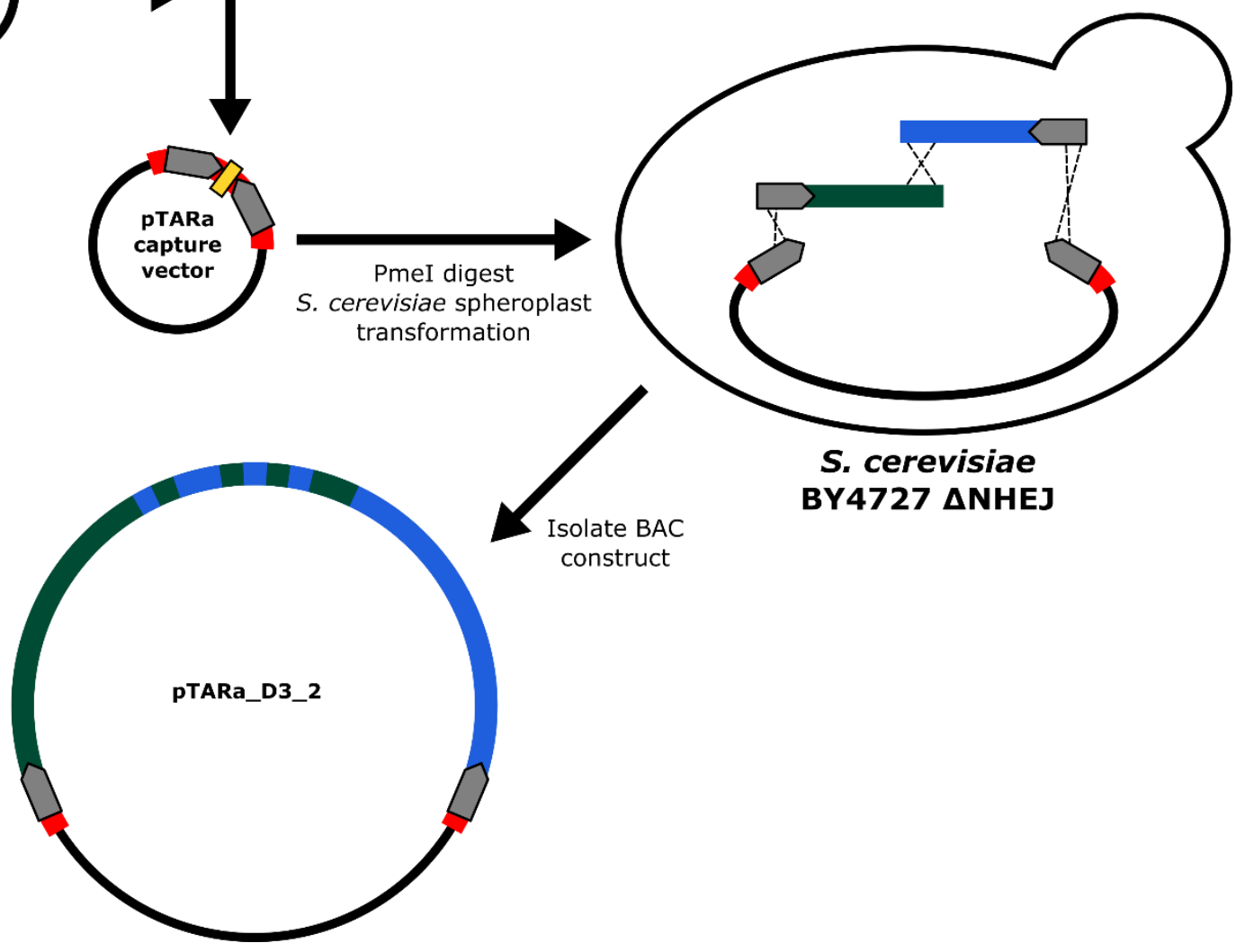

S. cerevisiae

BY4727 $\triangle$ NHEJ

Figure 6.5 - Transformation associated recombination scheme for reassembling the metagenomic aureolic acid biosynthetic gene cluster. Using the DNA sequence of cosmids pWEB::tnc_D3 and pWEB::tnc_D3_M13OL, homology "capture" arms (D3 UP and D3 DN) were designed, each having 712 bp of homology to the metagenome DNA sequence. These homology arms were synthesised in a DNA block with $35 \mathrm{nt}$ of homology to the pTARa vector (for Gibson assembly) and with a PmeI restriction site in between the capture arms to permit linearisation. The synthetic DNA block was Gibson assembled into pTARa at the NheI restriction site, and then linearised by PmeI digestion. The two pWEB::tnc cosmid inserts were liberated by Cas9 digestion, with CRISPR guide RNA oligos targeting the edge of the vector sequence. Linear pTARa capture vector and metagenome inserts were transformed into S. cerevisiae BY4727 $\triangle$ NHEJ spheroplasts, and assembled by in vivo homology recombination. The fully assembled BAC construct was then isolated from a $S$. cerevisiae culture. 
In order to assemble this gene cluster for heterologous expression, transformation associated recombination (TAR) cloning was employed (Figure 6.5). Broadly, the method described in Kim et al. 2010 and Kallifidas and Brady 2012 was followed ${ }^{111,114}$, with three major deviations. Instead of using the PCR TAR capture vector design strategy described in the previous works, I developed a new strategy using synthetic DNA blocks. This avoided complicated PCR steps, and has proven to be a cloning strategy amenable to construction of multiple capture vectors in parallel. Another deviation from the previous protocols was the use of in vitro CRISPR-Cas9 digestion of the cosmids rather than the type II restriction endonuclease digest strategy previously employed. This resulted in cutting the metagenome insert out of the cosmid vector very close to the TAR recombination sites, which likely increased the TAR efficiency. The final change was the use of a non-homologous-end-joining (NHEJ) knockout strain of S. cerevisiae. This reduced the number of "background" colonies that resulted from capture vector circularisation without cluster capture inside $S$. cerevisiae cells, again increasing the overall TAR cloning efficiency.

The TAR capture arm homology sites were designed from $712 \mathrm{bp}$ of the outside edges of the consensus sequences of the original D3 cosmid and the M13 overlap cosmid (Figure 6.5). These capture arm sequences were set around a PmeI restriction site (to linearise the capture vector prior to TAR assembly), and with $35 \mathrm{nt}$ of homology to the pTARa capture vector, for insertion via Gibson assembly. The designed DNA block was synthesised by Twist Biosciences. The two cosmids containing the biosynthetic gene cluster sequence were digested with Cas9 using custom CRISPR oligos designed to target the pWEB::tnc vector edge sequence. The linearised cosmid inserts, and pTARa capture vector were transformed into S. cerevisiae BY4727 $\triangle$ NHEJ spheroplasts, using the cited protocols 111,114 . Of the resulting $\sim 150$ colonies, 12 were initially patched onto fresh SC agar, and screened with a single PCR primer pair (D3_midd). All 12 restreaks tested positive, and four were then cultured in liquid SC media, with the BAC extracted by miniprep. This resulting construct was validated by PCR of five evenly spaced unique regions along the construct length, and with Sanger sequencing of various regions of the construct. All four preps were tested positive for correct assembly. Finally, the pTARa construct was fully sequenced by Illumina HiSeq 150bp PE, and this aligned perfectly to the theoretical assembly - including assembly breakdown in the repeat region outside of the gene cluster. 


\subsection{Aureolic acid gene cluster annotation}

The final confirmation of the biosynthetic gene cluster was based on the aligned sequences of the three recovered cosmid sequences, and the sequence of the resulting TAR construct, which were each sequenced separately and aligned in total agreement. The sequence was analysed using antiSMASH v4 and v5, with each encoded amino acid sequence then further analysed by BLASTp analysis against the aureolic acid gene clusters (mithramycin and chromomycin $\mathrm{A}_{3}$ ), the MIBiG database v1.4, and National Center for Biotechnology Information non-redundant protein sequences database (NCBI-nr) database (Figure 6.6 and Table 6.1). The amino acid identities for each gene, together with predicted biosynthetic functions, are listed in Table 6.1 relative to the corresponding mithramycin/chromomycin homologues.

The metagenome derived gene cluster has a length of $42,302 \mathrm{bp}$, similar to the mithramycin (42,374 bp) and chromomycin (42,074 bp) biosynthetic gene clusters. Of the genes that were assigned to homologous functions, the mean percentage amino acid identity was $60.7 \%$ to mithramycin and $52.7 \%$ to chromomycin. The metagenome pathway contains 5 open reading frames assigned by BLAST analysis as transposases. This is not uncommon for biosynthetic gene clusters, which are often mobile genetic elements propagated by horizontal gene transfer.

In general, the majority of genes in the metagenome pathway share the highest BLASTp identities with genes from the two aureolic acid gene clusters in the MIBiG database, however this is notably not the case for the first six enzyme functions in the biosynthetic pathway (core polyketide biosynthesis by $\mathrm{P}, \mathrm{K}, \mathrm{S}$, and cyclisation/aromatisation by X, Y, and Q). Only Q has its nearest homologue in chromomycin, while all other genes align to other, non aureolic acid biosynthesis. $\mathrm{P}$ and $\mathrm{S}$ align most strongly to genes involved in synthesis of urdamycin (angucycline), $\mathrm{K}$ to simocyclinone (angucycline), $\mathrm{X}$ to dutomycin (anthracycline), and Y to arimetamycin (anthracycline). 


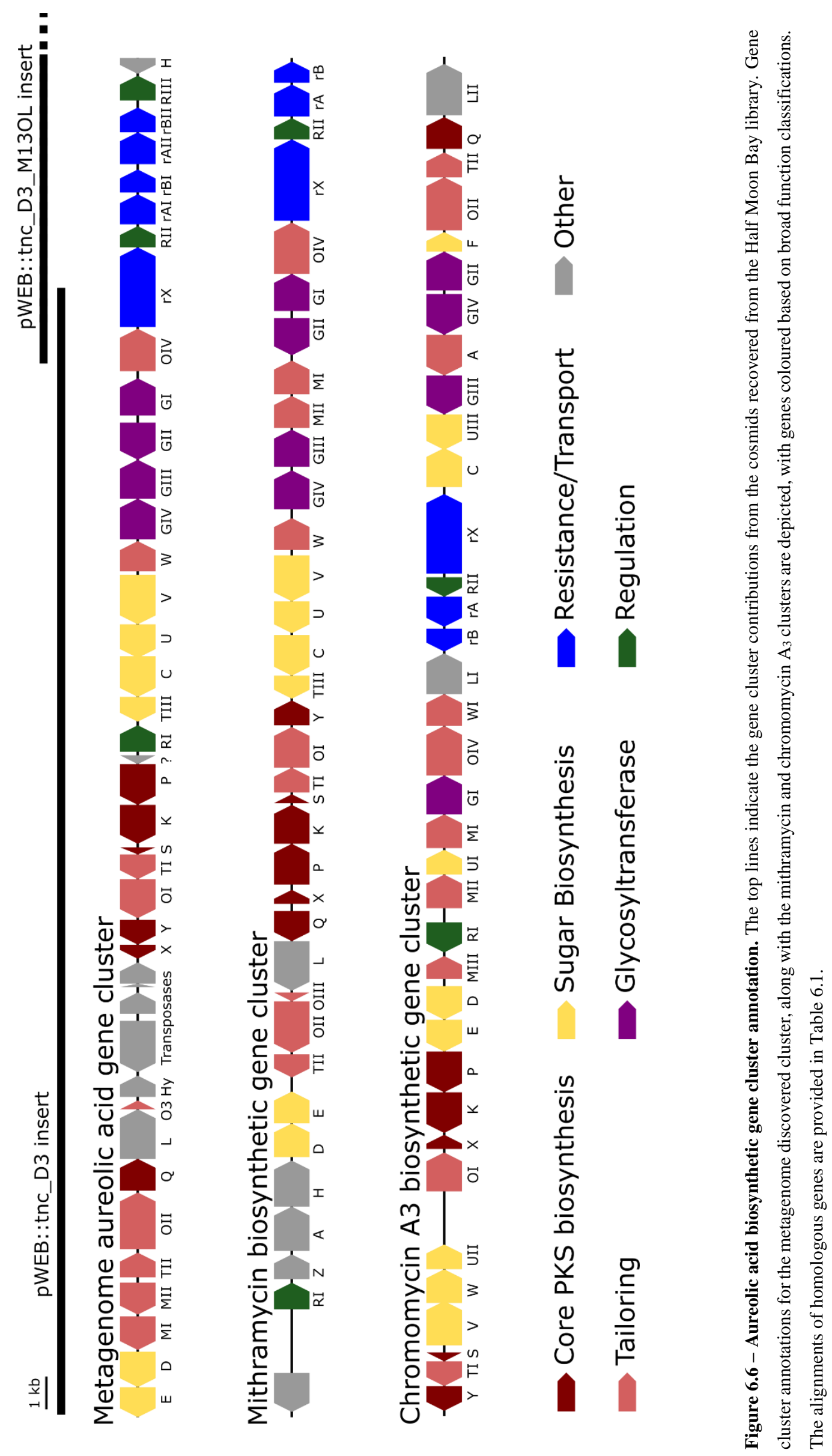




\begin{tabular}{|c|c|c|c|c|c|}
\hline & $\begin{array}{l}\text { Metagenome } \\
\text { pathway }\end{array}$ & $\begin{array}{l}\text { Mithramycin } \\
\text { pathway }\end{array}$ & $\begin{array}{l}\text { Chromomycin } \\
\text { A3 pathway }\end{array}$ & Proposed function & $\begin{array}{l}\text { \% aa identity } \\
\text { (D3:mithramycin } \\
\text { /chromomycin) }\end{array}$ \\
\hline A & & & $\mathrm{CmmA}$ & O-acyltransferase & \\
\hline A & & MtmA & & AdoMet synthetase & \\
\hline $\mathrm{C}$ & $\mathrm{C}$ & MtmC & $\mathrm{CmmC}$ & NDP-C-methyltransferase & $72.7 / 39.5$ \\
\hline $\mathrm{D}$ & $\mathrm{D}$ & MtmD & $\mathrm{CmmD}$ & NDP-glucose synthase & $53.8 / 53.0$ \\
\hline $\mathrm{E}$ & $\mathrm{E}$ & MtmE & $\mathrm{CmmE}$ & NDP-4,6-dehydratase & $63.4 / 65.8$ \\
\hline $\mathrm{F}$ & & & $\mathrm{CmmF}$ & NDP-5-epimerase & \\
\hline GI & GI & MtmGI & CmmGI & Glycosyltransferase & $57.1 / 38.9$ \\
\hline GII & GII & MtmGII & CmmGII & Glycosyltransferase & $58.4 / 42.2$ \\
\hline GIII & GIII & MtmGIII & CmmGIII & Glycosyltransferase & $66.9 / 46.0$ \\
\hline GIV & GIV & MtmGIV & CmmGIV & Glycosyltransferase & $67.6 / 47.7$ \\
\hline $\mathrm{H}$ & $\mathrm{H}$ & $\mathrm{MtmH}$ & & Adenosylhomocysteinase & $90.1 /$ \\
\hline Hy & Hy & & & Hydrolase & \\
\hline K & $\mathrm{K}$ & MtmK & $\mathrm{CmmK}$ & Ketosynthase $\beta$ & $63.5 / 64.4$ \\
\hline LI & & & CmmLI & Acyl-CoA ligase & \\
\hline LII & $\mathrm{L}$ & MtmL & CmmLII & Acyl-CoA ligase & $48.3 / 53.8$ \\
\hline MI & MI & MtmMI & CmmMI & O-methyltransferase & $56.1 / 55.1$ \\
\hline MII & MII & MtmMII & CmmMII & C-methyltransferase & $50.3 / 54.2$ \\
\hline MIII & & & CmmMIII & O-methyltransferase & \\
\hline OI & OI & MtmOI & CmmOI & Oxygenase & $54.1 / 56.8$ \\
\hline OII & OII & MtmOII & CmmOII & Oxygenase & $51.6 / 53.5$ \\
\hline OIII & & MtmOIII & & Oxygenase & \\
\hline $\mathrm{O} 3$ & $\mathrm{O} 3$ & & & Oxygenase & \\
\hline OIV & OIV & MtmOIV & CmmOIV & Oxygenase & $63.3 / 57.2$ \\
\hline $\mathrm{P}$ & $\mathrm{P}$ & $\mathrm{MtmP}$ & $\mathrm{CmmP}$ & Ketosynthase $\alpha$ & $67.6 / 71.9$ \\
\hline Q & $\mathrm{Q}$ & MtmQ & CmmQ & Aromatase & $50.2 / 55.9$ \\
\hline RI & RI & MtmR & CmmRI & Transcriptional activator & $41.7 / 41.6$ \\
\hline RII & RII & MtrY & CmmRII & Regulator & $67.4 / 23.7$ \\
\hline RIII & RIII & & & Regulator & \\
\hline S & S & MtmS & $\mathrm{CmmS}$ & Acyl carrier protein & $41.3 / 49.3$ \\
\hline TI & TI & MtmTI & CmmTI & Ketoreductase & $50.2 / 52.3$ \\
\hline TII & TII & MtmTII & CmmTII & Ketoreductase & $58.5 / 57.3$ \\
\hline TIII & TIII & MtmTIII & & NDP-4-ketoreductase & $63.4 /$ \\
\hline UI & & & CmmUI & NDP-4-ketoreductase & \\
\hline UII & & & CmmUII & NDP-4-ketoreductase & \\
\hline UIII & & & CmmUIII & NDP-4-ketoreductase & \\
\hline $\mathrm{V}$ & $\mathrm{V}$ & $\mathrm{MtmV}$ & $\mathrm{CmmV}$ & NDP-2,3-dehydratase & $61.3 / 46.2$ \\
\hline $\mathrm{U}$ & $\mathrm{U}$ & MtmU & $\mathrm{CmmW}$ & NDP-3-ketoreductase & $64.0 / 52.5$ \\
\hline $\mathrm{W}$ & $\mathrm{W}$ & MtmW & CmmWI & Ketoreductase & $75.1 / 65.4$ \\
\hline $\mathrm{X}$ & $\mathrm{X}$ & MtmX & $\mathrm{CmmX}$ & Cyclase & $52.6 / 57.0$ \\
\hline $\mathrm{Y}$ & $\mathrm{Y}$ & MtmY & $\mathrm{CmmY}$ & Cyclase & $67.7 / 70.4$ \\
\hline $\mathrm{Z}$ & & MtmZ & & Thioesterase & \\
\hline rAI & rAI & MtrA & $\mathrm{CmmA}$ & ATP-binding protein & $66.3 / 52.9$ \\
\hline rAII & rAII & & & ATP-binding protein & \\
\hline rBI & $\mathrm{rBI}$ & MtrB & $\mathrm{CmmB}$ & Membrane protein & $62.4 / 48.8$ \\
\hline rBII & rBII & & & Membrane protein & \\
\hline $\mathrm{rX}$ & $\mathrm{rX}$ & MtrX & $\mathrm{CmmX}$ & UV-repair system & $63.6 / 54.0$ \\
\hline
\end{tabular}


Annotation of the D3 biosynthetic pathway indicated that all of the genes were present necessary for biosynthesis of a tetracyclic aureolic acid aglycone that was similar or identical to premithramycinone (Figure 6.3). The other key genes implicated in biosynthesis (MII, GI-GIV, OIV and W) are also represented in the metagenome gene cluster, potentially facilitating the methylation and glycosylation of premithramycinone, followed by ring opening and sidechain reduction characteristic of aureolic acid biosynthesis. The genes that would be required for downstream modification to generate chromomycin $\mathrm{A}_{3}$ by $\mathrm{O}$-acylation (A) and O-methylation (MIII) are not present in the D3 metagenome cluster.

There are four glycosyltransferase genes present, indicating at least four sugars are likely to be present in the final molecule of the biosynthetic pathway. In both mithramycin and chromomycin $\mathrm{A}_{3}$, glycosyltransferase IV catalyses sugar transfer twice in compound biosynthesis, and all characterised aureolic acids have been four and six sugars over two oligosaccharide chains. The identity of these sugars represents a key difference between aureolic acid structures. All known aureolic acids contain combinations of D-olivose, Doliose and D-mycarose, with only chromomycin and olivomycin containing L-chromose B sugars. In mithramycin biosynthesis, enzymes MtmD, E, V, W, C, U and TIII are involved in the biosynthesis of D-olivose, D-oliose and D-mycarose. In chromomycin biosynthesis, enzymes CmmD, E, V, W, UI, UII, C, F and UIII are involved in the biosynthesis of Dolivose, D-oliose and L-chromose B. The metagenome gene cluster lacks the epimerase (F) and subsequent reductase (UIII) enzymes that would be required for the production of Lchromose B. The lack of a sugar epimerase gene indicates that all glycosylated metabolites of the metagenome pathway expression should contain only D-sugars. The metagenome cluster contains homologues indicative of a full sugar biosynthetic pathway for D-olivose, D-oliose and D-mycarose (Figure 6.7). 


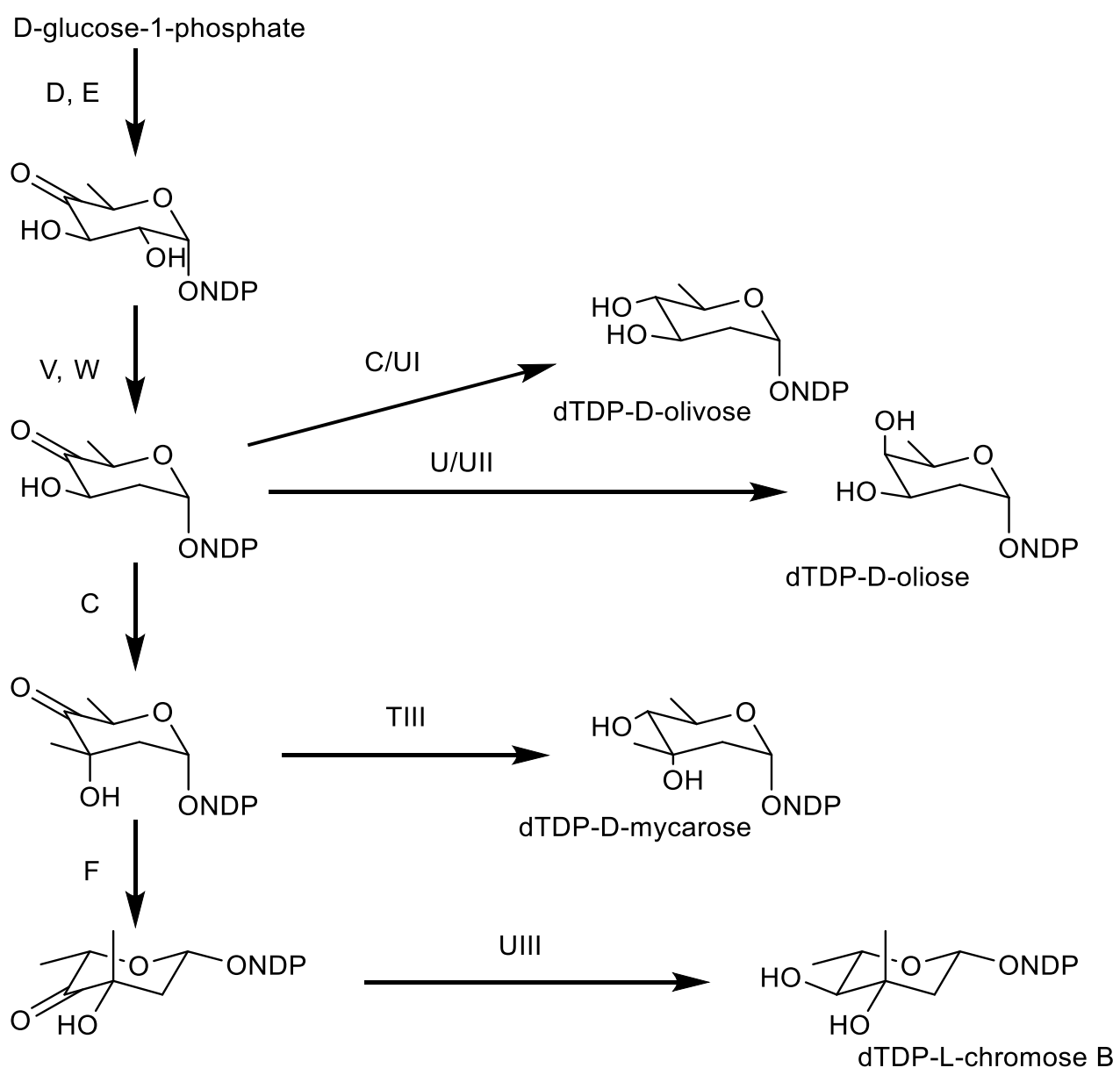

Figure 6.7 - Biosynthetic pathways for production of deoxy sugars in aureolic acid biosynthesis. The metagenome derived gene cluster in this study contains homologues for genes D, E, V, W, C, U and TIII. These genes may function to permit the biosynthesis of dTDP-D-olivose, oliose, and mycarose.

The metagenome cluster contains a few notable genes that differ from either of the known aureolic acid biosynthesis pathways, which may result in different metabolic output. The third oxygenase in the pathway (O3) does not have significant sequence homology to MtmOIII (chromomycin lacks a third oxygenase). The O3 gene has some homology to the DutO3 monoxygenase in dutomycin biosynthesis (51.6\% amino acid identity), as the nearest homologue in the MIBiG database. The metagenome gene cluster contains a HAD family hydrolase gene (Hy), which shares low-level identity with a putative hydrolase in streptolydigin biosynthesis (56.4\% amino acid identity). Also present is an extra regulatory protein (RIII) that aligns by BLASTp to TetR regulatory proteins. There are also twice as many drug efflux genes present in the metagenome pathway; whereas the mithramycin and chromomycin gene clusters both contain a pair of A/B genes, homologues of each of these genes are present twice in the metagenome pathway. However, these genes do not appear 
to be the result of a recent duplication event, as the two ATP-binding components of the $\mathrm{ABC}$ transporter (rAI/rAII) have only a $68.1 \%$ nucleotide pairwise alignment identity. Likewise the membrane protein components (rBI/rBII) have a $66.2 \%$ nucleotide pairwise identity.

\subsection{Heterologous expression studies}

The BAC clone containing the full genetic pathway was transferred to $S$. albus for heterologous expression. This process involved first transferring the BAC from $S$. cerevisiae to E. coli $\mathrm{EPI} 300$ for propagation, then to the E. coli S17.1 conjugation strain, then finally conjugating the BAC into $S$. albus, where the pathway was chromosomally integrated. Two strains of S. albus were used for conjugation - S. albus $\mathrm{J} 1074$ and S. albus B4 - a strain of S. albus J1074 in which all endogenous biosynthetic gene clusters have been knocked out and additional integration sites inserted ${ }^{77}$. At each stage in the process the integrity of the DNA construct was validated using the previously mentioned 5 PCR primer sets.

Once the construct had been transferred to the final recipient strains, culture spore stocks were made and expression trials were conducted. Cultures were initially grown in TSB medium, and after 24-48 hours of growth, used to seed R5a medium cultures (1\% inoculum), with or without the addition of $20 \mathrm{~g} / \mathrm{L}$ HP20 resin. The HP20 resin was included with the growth media to collect metabolites as the culture proceeded.

Cultures were grown for 7-10 days, after which HP20 resin was collected from mature and bound metabolites eluted in 100\% methanol. Cultures without added HP20 were extracted with an equal volume of ethyl acetate. A second ethyl acetate extraction was then performed by adding $\mathrm{HCl}$ to decrease the aqueous phase $\mathrm{pH}$ to $2-3$, to allow recovery of metabolites that require protonation to move into ethyl acetate.

Extracts from these expression trials were analysed by HPLC, with a DAD monitoring 200$800 \mathrm{~nm}$ spectrum range, and by LCMS-Qtof. The characteristic aureolic acid UV signature 187,200 was used to guide metabolite peak identification (Figure 6.8). The primary findings of these experiments were that the major metabolite of this pathway had an $\mathrm{m} / \mathrm{z}$ of 975.3848 $[\mathrm{M}-\mathrm{H}]^{-}$, (compound 4, corresponding to $\mathrm{C}_{48} \mathrm{H}_{64} \mathrm{O}_{21}[\mathrm{M}-\mathrm{H}]^{-}=975.3867$ ). This is the same molecular formula as the known aureolic acid chromocyclomycin. However, the HPLC traces had many other small peaks with the same distinctive aureolic acid UV spectrum, 
particularly in the HP20 extract (Figure 6.8). Fewer pathway associated metabolites were observed in the ethyl acetate extracts. The extracts from S. albus B4 contained far fewer pathway associated compounds, and the peaks were far smaller, therefore S. albus J1074 was used as the expression strain going forward.

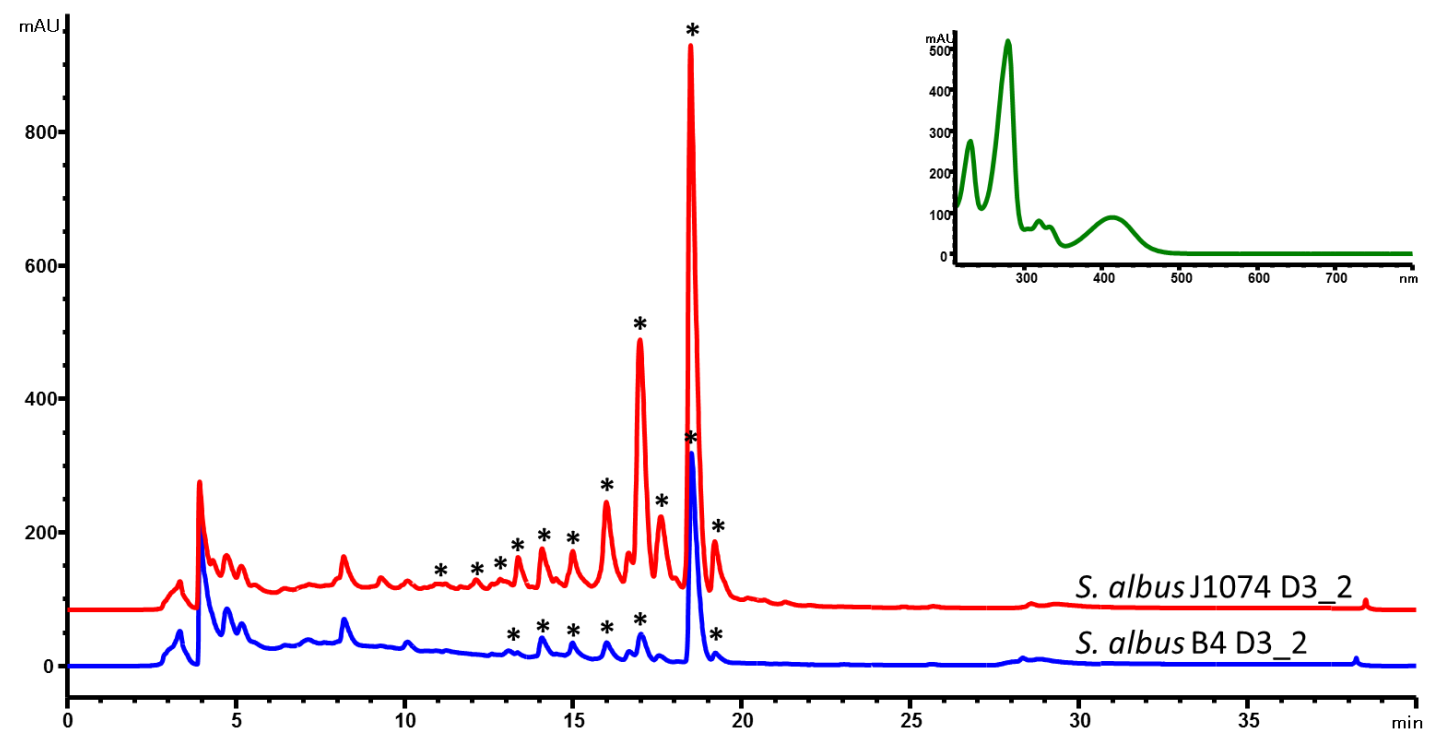

Figure 6.8 - Expression of complete D3_2 metagenome aureolic acid pathway. HPLC traces of HP20 extracts of mature expression cultures in S. albus J1074 (red) and S. albus B4 (blue). Metabolite peaks with the characteristic aureolic acid UV absorbance spectrum (inset, green) are indicated with an asterisk (*). Compound $\mathbf{4}$ is the major metabolite peak, with a retention time of 18.5 minutes. The retention time on the reversed-phase $\mathrm{C}_{18}$ column is shown on the $\mathrm{X}$-axis, and absorbance at $280 \mathrm{~nm}$ on the $\mathrm{Y}$-axis.

Zone of inhibition assays were conducted on the extracts, and I found that crude methanolic extract eluted from the HP20 resin of the producing strain possessed antibacterial activity against test strain B. subtilis 168 where wild type S. albus did not (Table 6.2). This antibacterial activity was not associated with the major product of the pathway (compound 4), as minimal purification of this peak by HPLC resulted in loss of antibacterial activity. I hypothesised the observed bioactivity was linked to one of the small peaks on the HPLC trace that had aureolic acid type UV absorbance. 
Zone of inhibition test strains

\begin{tabular}{|c|c|c|c|c|}
\hline Expression strain & Extract & $\begin{array}{l}\text { B. subtilis } \\
168\end{array}$ & E. coli 7NT & $\begin{array}{l}\text { S. } \\
\text { cerevisiae }\end{array}$ \\
\hline \multirow[t]{3}{*}{ S. albus J1074 } & Ethyl acetate & + & + & + \\
\hline & $\begin{array}{l}\text { Acidified ethyl } \\
\text { acetate }\end{array}$ & + & + & ++ \\
\hline & HP20 & - & + & ++ \\
\hline \multirow{3}{*}{$\begin{array}{l}\text { S. albus J1074 } \\
\text { pTARa_D3_2 }\end{array}$} & Ethyl acetate & + & + & + \\
\hline & $\begin{array}{l}\text { Acidified ethyl } \\
\text { acetate }\end{array}$ & ++ & + & ++ \\
\hline & HP20 & ++ & + & ++ \\
\hline Negative control & & - & - & - \\
\hline Positive control & & ++ & ++ & ++ \\
\hline
\end{tabular}

Table 6.2 - Preliminary zone of inhibition screening of crude culture extracts against three test strains. Bioactivity scoring was ranked into three categories, "-“ being a similar result to the negative control (no zone of inhibition), "++" being a similar result to the positive control (clear zone of growth inhibition), and "+" an intermediate result. The negative control was DMSO in all cases, and positive antibiotic controls were tetracycline for B. subtilis and E. coli test strains, and nystatin for S. cerevisiae.

The HP20 extract was then fractionated on an HPLC gradient from 10-100\% acetonitrile $/ \mathrm{H}_{2} \mathrm{O}(0.1 \%$ formic acid $)$ over 35 minutes, with automatic fraction collection at 1 minute intervals. Fractions were dried completely, resuspended in DMSO, and spotted onto LB agar infused with the antibiotic test strain B. subtilis 168 to observe potential antibacterial function. The fractions where bioactivity was observed were pooled and refractionated on a longer, shallower gradient. After three round of this process, culminating in a HPLC gradient of $30-65 \%$ acetonitrile $/ \mathrm{H}_{2} \mathrm{O}(0.1 \%$ formic acid $)$ over 70 minutes, a relatively pure sample containing an ion with $\mathrm{m} / \mathrm{z} 967.4186[\mathrm{M}-\mathrm{H}]^{-}$was isolated that possessed potent bioactivity (compound 5, corresponding to $\mathrm{C}_{47} \mathrm{H}_{68} \mathrm{O}_{21}[\mathrm{M}-\mathrm{H}]^{-}=$ 967.4180). Notably, the mass difference between compound 4 and compound 5 (976.3928 - $968.4256=7.9672$ ) is the same mass difference between premithramycin B and mithramycin $(1092.4414-1084.4727=7.9687)$, indicating a similar chemical transformation.

MS/MS analysis of compound $\mathbf{5}$ and the major biosynthesis product compound $\mathbf{4}$ against a purchased sample of authentic mithramycin was then conducted (Figure 6.9). Compound 5 fragmented similarly to the mithramycin standard, with some of the fragmented ions shifted by a mass corresponding to the loss of a deoxy sugar and the gain of a methyl group (consistent with the mass difference between mithramycin and compound 5). The observed 
fragmentation pattern in both cases initiated with the loss of the aliphatic sidechain (adduct ion $\mathrm{m} / \mathrm{z} 820$ or 935 ) at $20.0 \mathrm{eV}$, then successive loss of deoxy sugars up to $60 \mathrm{eV}$, leaving the tricyclic core at m/z 269 (extended MS/MS spectra in Appendix E). The observed mass and fragmentation patterns of compound $\mathbf{5}$ are consistent with a "ring opened" aureolic acid (Figure 6.9-B). This fragmentation pathway was consistent with the gene cluster analysis, where the presence of OIV and $\mathrm{W}$ homologues indicate a ring opening step should occur in the biosynthesis pathway (Figure 6.6, Table 6.1) as for mithramycin and chromomycin $\mathrm{A}_{3}$ (Figure 6.3). Compound 4 did not follow the same fragmentation pattern as the mithramycin standard, potentially due to the lack of an aliphatic tail where the initial fragmentation occurs (at $20.0 \mathrm{eV}$ onwards). Instead, the compound 4 ion remained unfragmented at $20.0 \mathrm{eV}$, with minor fragmentation at $40.0 \mathrm{eV}$, followed by seemingly disordered breakdown at $60.0 \mathrm{eV}$, including the conserved core masses observed in mithramycin/compound 5 MS/MS (Figure 6.9-C). This breakdown pattern is potentially indicative of a resistant cyclised structure for compound 4. 

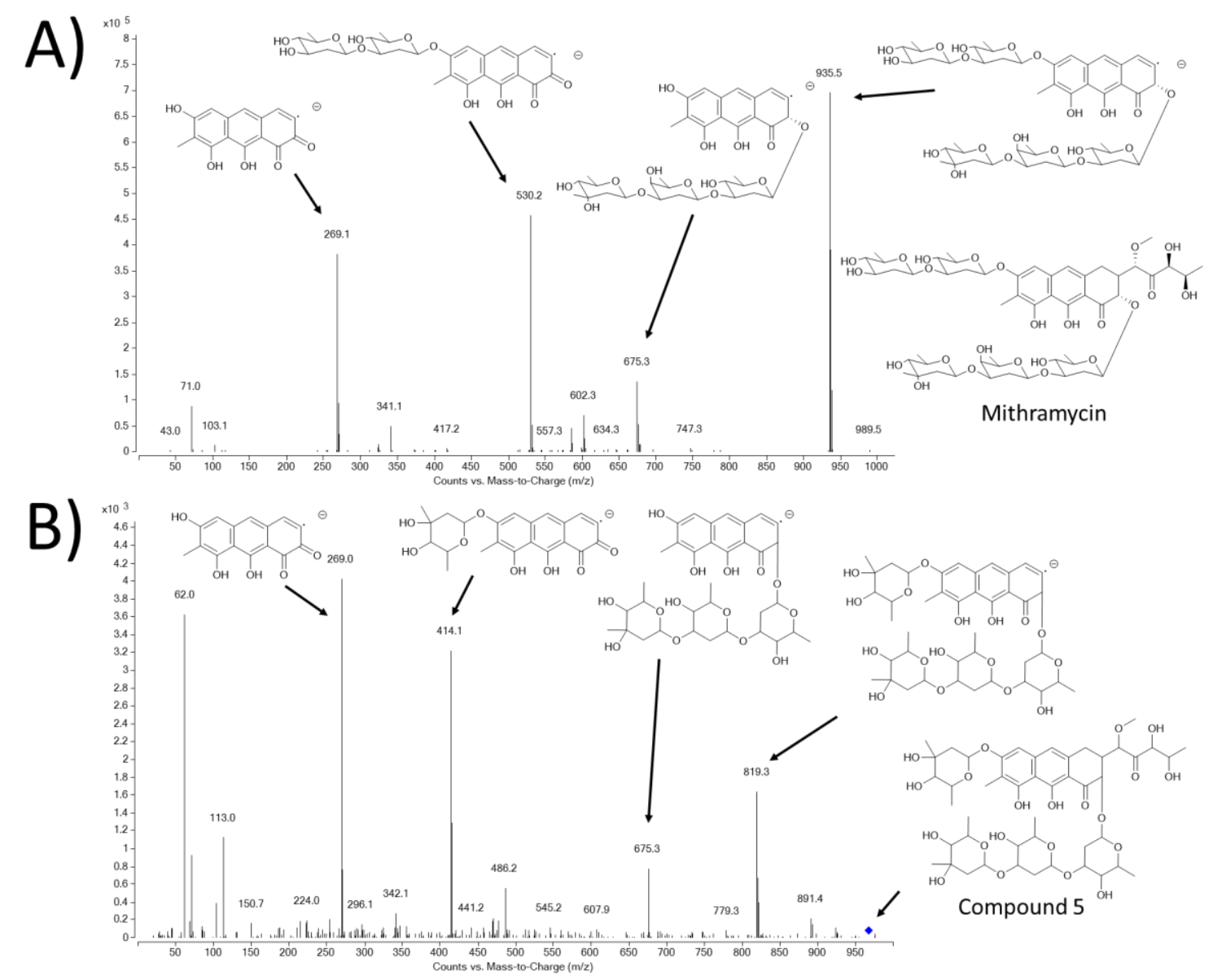

C)

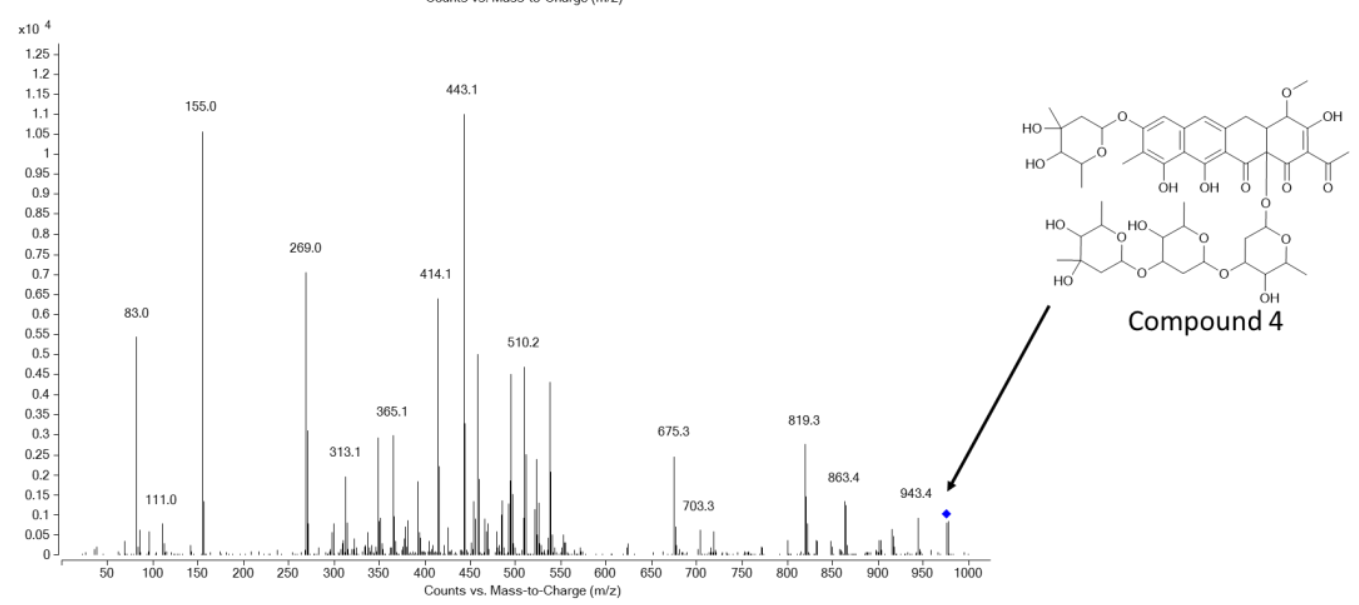

Figure 6.9 - MS/MS spectra of a mithramycin standard, compound 4 and compound 5. All the depicted MS/MS fragmentation analysis was conducted at $60.0 \mathrm{eV}$. A) Tandem MS showing a possible fragmentation patterns from a sample of mithramycin. Two of the observed adducts also occur in panel B. B) Tandem MS fragmentation pattern of compound 5. The non-conserved adduct $\mathrm{m} / \mathrm{z}$ differ by 116 (i.e. $935-819$ and 530-414), equivalent to the mass difference between mithramycin and compound 5. C) Tandem MS analysis for compound $\mathbf{4}$ does not follow a similar fragmentation pattern to mithramycin or compound $\mathbf{5}$, although some conserved adduct ion $\mathrm{m} / \mathrm{z}$ are present $(819,675,414$ and 269$)$ in amongst a disordered breakdown. The proposed structures for compound $\mathbf{4}$ and compound $\mathbf{5}$ are inset and further described in section 6.6. 
In order to provide further evidence that compound $\mathbf{5}$ is a "ring opened" form of compound 4, the two genes linked to ring opening (OIV and W) were amplified from the pathway using PCR, and Gibson cloned into pIJ10257 as a bi-cistronic unit under control of the constitutive expression promoter ermE (Figure 6.10). The PCR primers were designed to include a unique Shine-Dalgarno sequence ${ }^{201}$ as a point of homology between the two genes to complete the assembly and allow for expression from a single promoter sequence. The resulting construct was then re-integrated into the $S$. albus::D3_2 strain (i.e. in addition to the full pathway). LCMS-QTOF analysis of expression studies found the ermE_OIV_W cassette resulted in a 1.9 fold increase in compound $\mathbf{5}$ production compared to the base expression strain (Figure 6.10). This result lends more evidence to support the hypothesis that compound 4 is not the (sole) final product of the biosynthesis pathway, and that compound $\mathbf{4}$ may be converted to compound $\mathbf{5}$ by the same mechanism as premithramycin B conversion to mithramycin (Figure 6.10).

The increase in conversion to compound $\mathbf{5}$ did not provide sufficient compound to isolate for full structure elucidation and bioactivity testing, as it was still a minor metabolite of the S. albus expression culture. Compound $\mathbf{4}$ was isolated in large quantities however (as the major metabolite product of the pathway), and this process is discussed in the following section. 
A)
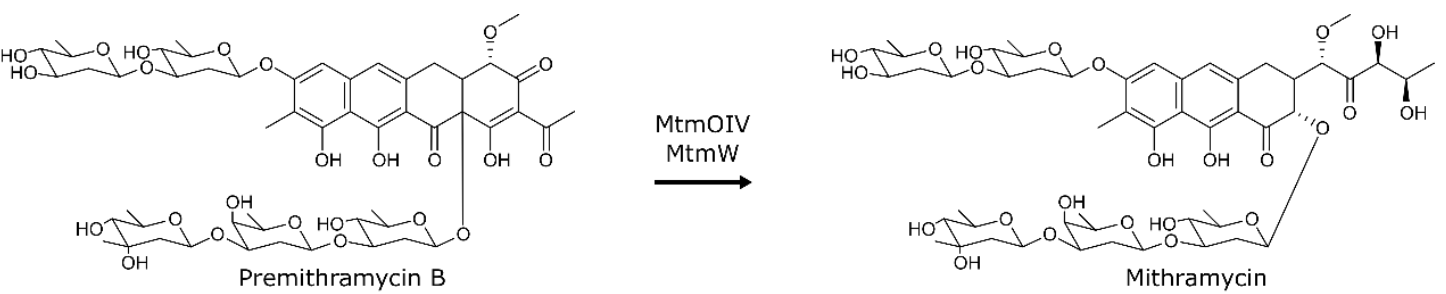

B)
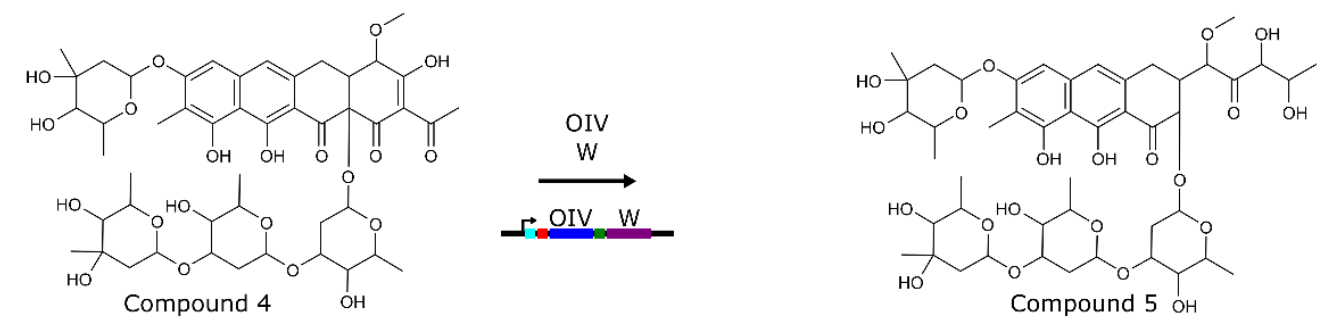

C)

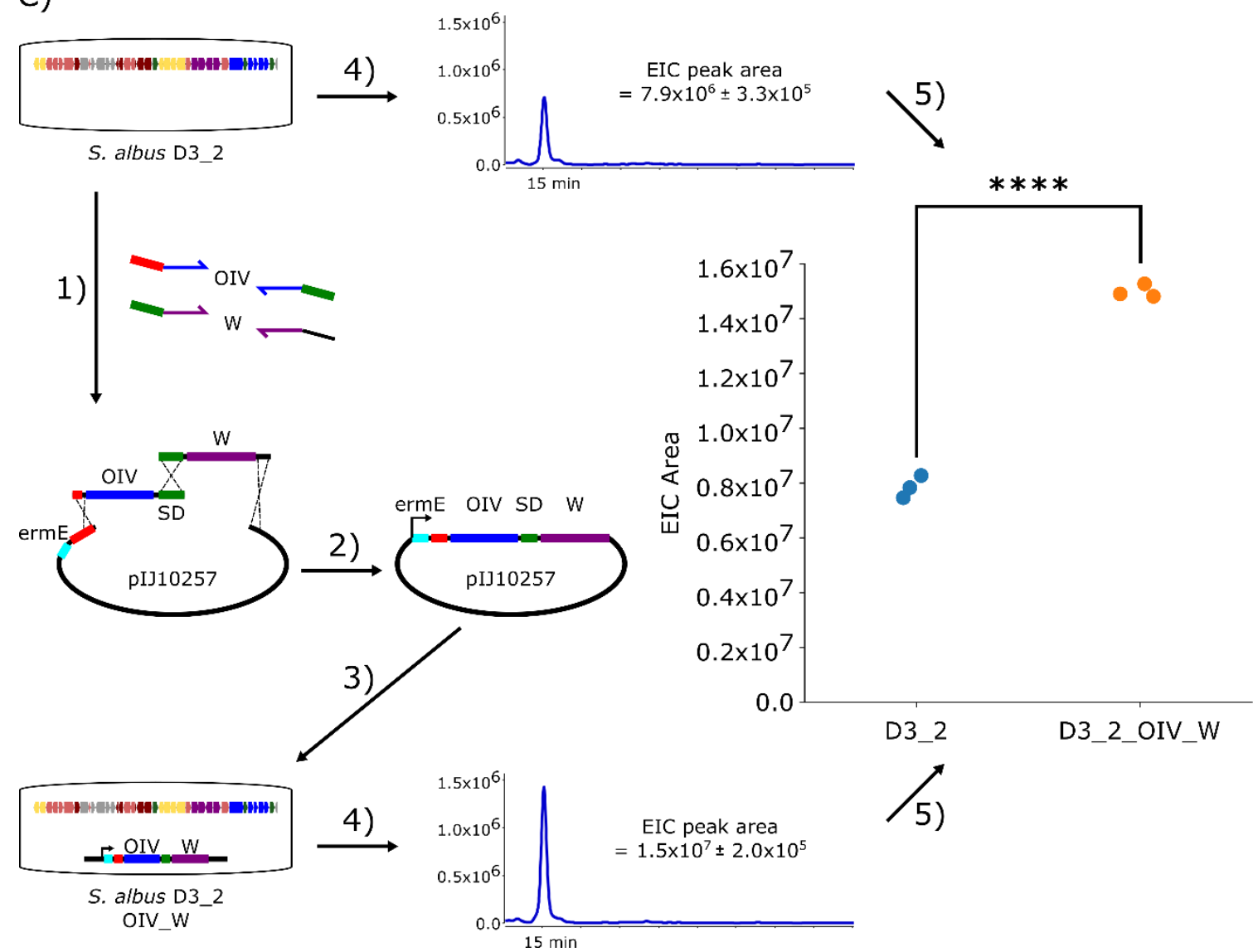

Figure 6.10 - Constitutive expression of ring OIV_W cassette for production of "ring opened" compound 5. Biosynthetic transformation by OIV, W enzymes in A) the mithramycin and B) the metagenome gene cluster. C1) Metagenomic pathway genes with sequence homology to mithramycin/chromomycin genes involved in ring opening were amplified from the pathway using PCR, with primers possessing homology to pIJ10257, and a unique ShineDalgarno sequence (green), for (2) Gibson cloning to form a bi-cistronic expression cassette under control of the constitutive expression promoter ermE (cyan). 3) This construct was integrated into the S. albus D3_2 expression strain, and LCMS analysis of the metabolites was conducted. 4) Extracted ion chromatograph (EIC) of the compound 5 [M-H] $\mathrm{m} / \mathrm{z}$ demonstrated an increase in expression of 1.9 fold over the D3_2 expression strain $(\mathrm{n}=3)$. 5) Student's t-test demonstrated a statistically significant difference of this metabolite production between the two strains $(\mathrm{p}=0.000013$ ). 


\subsection{Isolation and structural elucidation of compound 4}

To isolate sufficient quantities of compound 4 for structural elucidation studies, $7 \mathrm{~L}$ of $S$. albus::D3_2 expression culture in R5a with HP20 resin was established. The metabolite bound HP20 resin was collected from the culture medium at a final volume of $500 \mathrm{~mL}$, and loaded into a benchtop column. The HP20 was washed with $1 \mathrm{~L} \mathrm{of} \mathrm{H}_{2} \mathrm{O}$, and $1500 \mathrm{~mL}$ of $20 \%$ methanol $/ \mathrm{H}_{2} \mathrm{O}$, before elution in $1500 \mathrm{~mL} \mathrm{80 \%} \mathrm{methanol/} \mathrm{H}_{2} \mathrm{O}$. The $1500 \mathrm{~mL}$ elution was dried under reduced pressure, resuspended in $80 \mathrm{~mL}$ of methanol, and undissolved particulate removed via centrifugation. The clarified supernatant was dried, with a mass of $1091 \mathrm{mg}$. Half of this sample was then resuspended in methanol, syringe filtered, and partitioned on an LH20 column run in methanol, with $5 \mathrm{~mL}$ fractions collected and combined based on MS analysis for the presence of the compound 4 molecular ion. Four early fractions were combined, and were dried under reduced pressure to a dry mass of 262 mg. This sample was purified by semi-preparative $\mathrm{C}_{18}$ HPLC with a gradient of 10-100\% methanol $/ \mathrm{H}_{2} \mathrm{O}(0.2 \%$ formic acid $)$ over 60 minutes. Compound 4 had a retention time of 52.3 minutes, and $19.7 \mathrm{mg}$ was purified. A $10 \mathrm{mg}$ sample of this was then purified by analytical $\mathrm{C}_{18} \mathrm{HPLC}$ on an isocratic method of $75 \%$ methanol/ $\mathrm{H}_{2} \mathrm{O}(0.2 \%$ formic acid $)$, to give the final $7.9 \mathrm{mg}$ pure sample. This represents a recovery of $4.4 \mathrm{mg}$ per L of culture (when accounting for the unprocessed sample at each stage of purification).

While compound $\mathbf{4}$ had a predicted HR-MS derived mass value and molecular formula matching that of the known aureolic acid compound chromocyclomycin $\left(\mathrm{C}_{48} \mathrm{H}_{64} \mathrm{O}_{21}\right.$, monoisotopic mass $=976.39$ ), the MS/MS fragmentation analysis was uninformative as to this compound's structure (Figure 6.9). As there had been no recent studies into chromocyclomycin 187,202,203 we conducted our own NMR structure elucidation of this isolated compound. As we had evidence linking the structure of compound $\mathbf{4}$ to compound 5 (Figure 6.9, Figure 6.10), we sought to use the NMR validated structure of compound 4 to predict the structure of compound 5. The NMR studies were conducted, and the structure of compound 4 deduced by $\mathrm{PhD}$ candidate Joe Bracegirdle from Victoria University of Wellington School of Chemical and Physical Sciences.

The aglycone structure was confirmed by COSY and HMBC correlation as being consistent with the structure predicted by the biosynthesis (Figure 6.11). The aglycone structure of compound $\mathbf{4}$ is identical to the aglycone of chromocyclomycin, which is chromocyclin ${ }^{203}$. 
This structure is also consistent with the observed UV absorbance trace, MS, and MS/MS analysis.

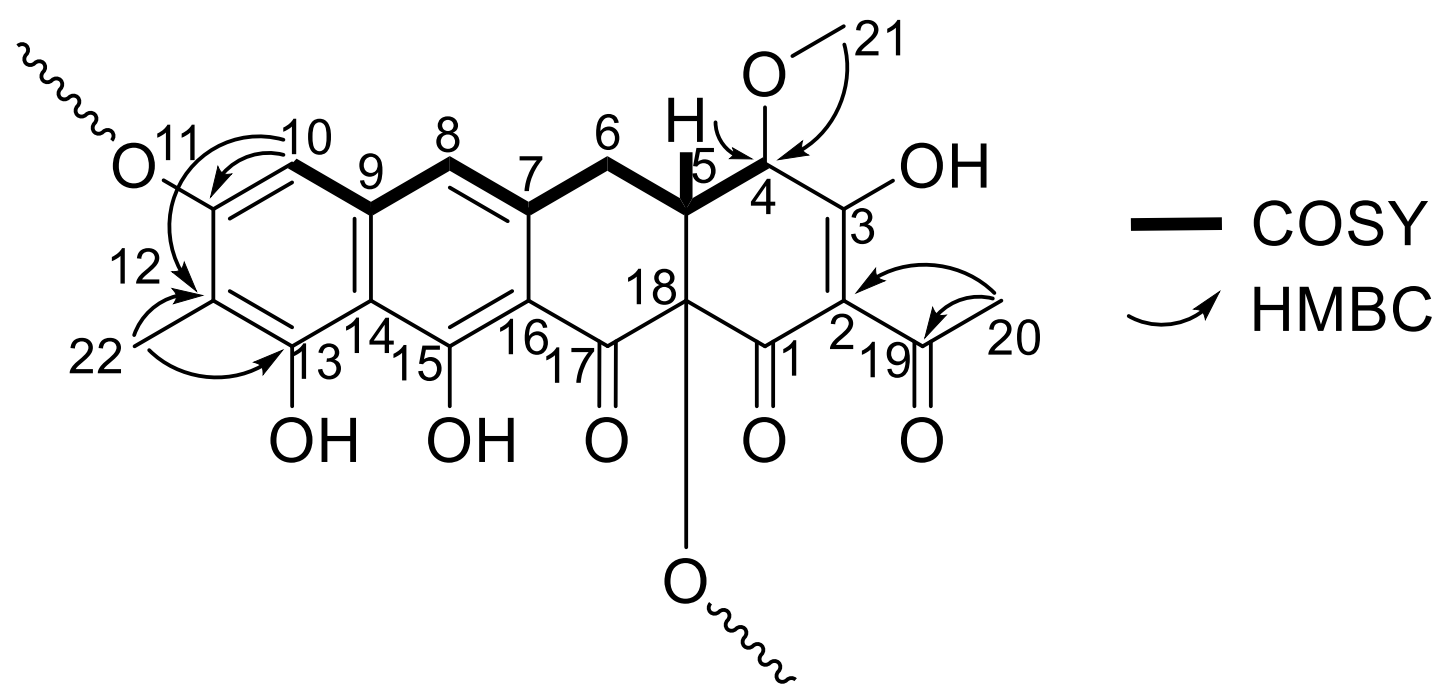

Figure 6.11 - Key NMR correlations by HMBC and COSY for the structure elucidation of the compound 4 aglycone.

Joe Bracegirdle then used more advanced NMR techniques to assign the sugar ${ }^{13} \mathrm{C}$ and ${ }^{1} \mathrm{H}$ resonances, determining the identity and arrangements of the four deoxy sugars and their glycosidic linkages (Figure 6.12). From this analysis he concluded that the sugars present are the same identity as for chromocyclomycin (a trisaccharide chain of D-olivose, Doliose, D-mycarose, and a monosaccharide D-mycarose), however in compound $\mathbf{4}$ these sugars are all $\beta$-glycosidically linked, whereas in chromocyclomycin both of the Dmycarose sugars are in the $\alpha$ configuration ${ }^{187,204}$. The trisaccharide chain is therefore identical to that of mithramycin ${ }^{200}$, while the monosaccharide is a $\beta$-linked mycarose, unlike any previously observed aureolic acid. This was confirmed by the large coupling constants $(J 9.6-9.7 \mathrm{~Hz})$ of the anomeric protons in each sugar, which is only observed in $\beta$-linked sugars as a result of the axial-axial relationship to one of the protons on the neighbouring methylene. This is consistent with both the published data on mithramycin $\beta$ deoxy sugar linkages ${ }^{200}$ and our independent analysis of a mithramycin standard. 


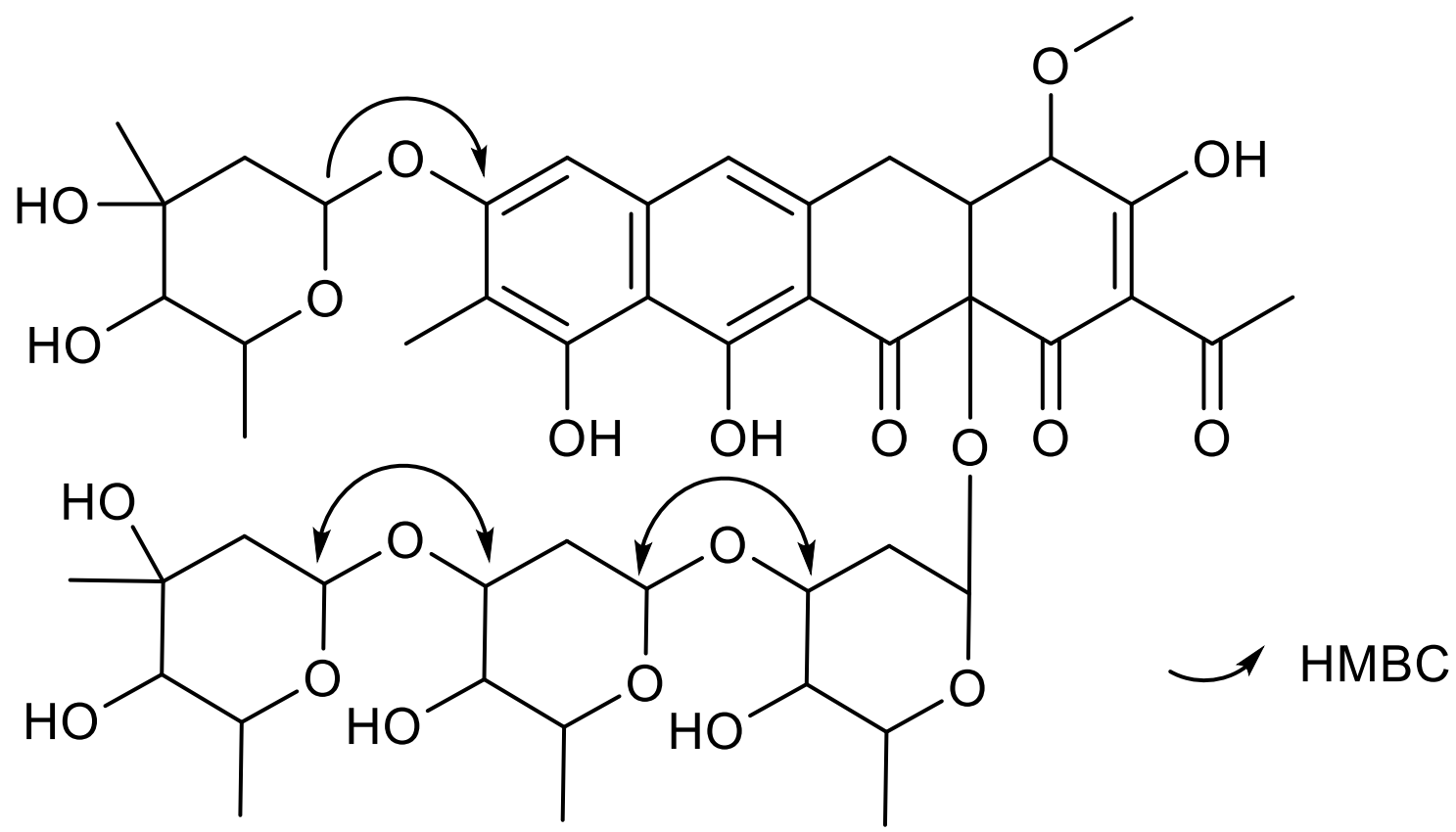

Figure 6.12 - Key NMR correlations and coupling relationships for determination of saccharide chain order in compound 4.

The proposed structure is consistent with the previously discussed data (biosynthetic gene cluster, established biosynthetic pathways, UV trace, MS, MS/MS, and enzyme overexpression studies), to confirm not only the structure of compound $\mathbf{4}$, but to indicate the observed glycosylation pattern is present in compound $\mathbf{5}$ also. The sugar chains on compound $\mathbf{4}$ and $\mathbf{5}$ are a monosaccharide D-mycarose, and a trisaccharide D-olivose, Doliose, D-mycarose, with all $\beta$-glycosidic linkages. In chromocyclomycin, the mono- and trisaccharide chains contain the same sugars, however the two D-mycarose sugars are $\alpha$ linked. Mithramycin contains the same trisaccharide chain as compounds 4 and 5 (Dolivose, D-oliose, D-mycarose), however it contains a disaccharide chain of two D-olivose sugars, where compounds $\mathbf{4}$ and $\mathbf{5}$ contain a single D-mycarose. Compounds $\mathbf{4}$ and $\mathbf{5}$ are both novel chemical entities, and represent new additions to the aureolic acid compound class. 
<smiles></smiles>

Compound 4

Chromocyclomycin<smiles>COC(C(=O)C(O)C(C)O)C1Cc2cc3cc(OC4CC(C)(O)C(O)C(C)C4C)c(C)c(O)c3c(O)c2C(=O)C1OC1CC(O)C(C)C(OC2CC(OC3CC(C)(O)C(O)C(C)C3O)C(C)O2)C1</smiles>

Compound 5

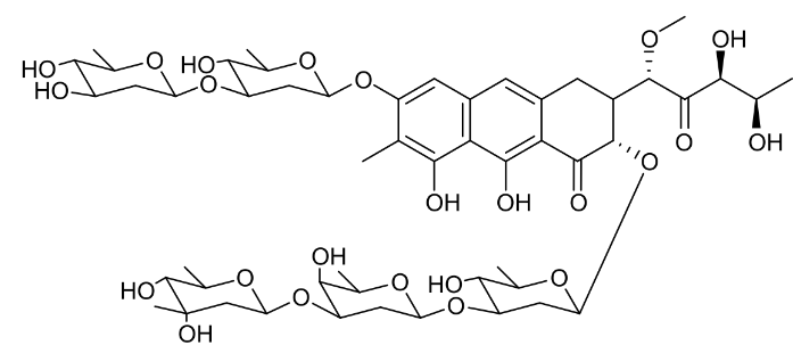

Mithramycin

Figure 6.13 - Structural comparison of compound 4 and 5 to the most closely related aureolic acids.

\subsection{Bioactivity assays}

While preliminary studies indicated that the major metabolite of the metagenome aureolic acid pathway (compound 4) did not possess antibacterial activity against the test strain $B$. subtilis 168, other studies into aureolic acids had found potent bioactivity against both Gram-positive bacterial strains and human cancer cell lines ${ }^{49,188,197,202}$. I investigated the biological activity of the isolated major metabolite (compound 4) against a panel of targets, including single representatives of Gram-positive bacteria (B. subtilis 168), Gram-negative bacteria (E. coli $7 \mathrm{NT})$, fungi (S. cerevisiae $\triangle P D R$ ), and human tumour cells (HCT-116). In all cases, a mithramycin A standard was used as a positive control antibacterial agent, along with tetracycline (for bacterial assays) and nystatin (for fungal testing). Dr Abigail Sharrock performed the human tumour cell bioactivity assays. 
Compound 4 Tetracycline Nystatin Mithramycin A

\begin{tabular}{l|ll|l|l|ll}
\hline Test strain & $\begin{array}{l}\text { MIC } \\
(\mu \mathrm{g} / \mathbf{m L})\end{array}$ & $\begin{array}{l}\mathbf{I C}_{50} \\
(\boldsymbol{\mu} \mathbf{M})\end{array}$ & $\begin{array}{l}\mathbf{M I C} \\
(\boldsymbol{\mu g} / \mathbf{m L})\end{array}$ & $\begin{array}{l}\text { MIC } \\
(\boldsymbol{\mu g} / \mathbf{m L})\end{array}$ & $\begin{array}{l}\text { MIC } \\
(\boldsymbol{\mu g} / \mathbf{m L})\end{array}$ & $\begin{array}{l}\mathbf{I C}_{50} \\
(\boldsymbol{\mu} \mathbf{M})\end{array}$ \\
\hline E. coli 7NT & $>128$ & & 0.125 & & $>32$ & \\
B. subtilis 168 & 2 & 1 & 0.5 & 0.004 & \\
S. cerevisiae $\Delta \mathrm{PDR}$ & 32 & & & 32 & \\
HCT-116 & & 1.91 & & & & 0.017 \\
\hline
\end{tabular}

Table 6.3 - Bioactivity assay results of antimicrobial and cytotoxicity assays. Antimicrobial minimum inhibitory concentration (MIC) assays were conducted for compound $\mathbf{4}$ with positive controls of tetracycline (antibacterial), nystatin (antifungal) and mithramycin A (Gram-positive antibacterial). Tumour cell cytotoxicity was performed with mithramycin A as a positive control agent. The data in the table were determined across at least three independent experiments.

Compound 4 was not found to possess any Gram-negative antibiotic activity, consistent with previously characterised aureolic acids. It does, however, possess moderate/strong Gram-positive antibiotic activity (B. subtilis 168 , MIC $2 \mu \mathrm{g} / \mathrm{mL}$ ). The MIC was twice that was observed for the positive control antibiotic tetracycline (B. subtilis $168 \mathrm{MIC} 1 \mu \mathrm{g} / \mathrm{mL}$ ), however on a molar basis the MIC values were similar; compound 4 inhibited growth at $2.05 \mu \mathrm{M}$, while tetracycline inhibited at $2.25 \mu \mathrm{M}$. The mithramycin A standard, however, had an MIC of $0.004 \mu \mathrm{g} / \mathrm{mL}$, indicating a 500-fold increase in Gram-positive antibacterial potency relative to compound 4 . This data may explain why compound $\mathbf{4}$ initially appeared to possess no significant Gram-positive antibacterial activity; prefractionated culture extracts containing compounds $\mathbf{4}$ and $\mathbf{5}$ produced large zones of inhibition on agar plates, whereas a crude separation of compound 4 did not, despite the greater abundance of compound $\mathbf{4}$ in those extracts. It is likely that compound $\mathbf{5}$ possesses Gram-positive antibacterial activity more comparable to mithramycin A than to compound 4 .

Compound 4 and mithramycin A both possessed weak-moderate antifungal bioactivity against the $S$. cerevisiae $\triangle \mathrm{PDR}$ strain. This is interesting, as the only previously reported antifungal activity of an aureolic acid was for durhamycin A, which was also reported to possess no activity against $B$. subtilis ${ }^{205}$. However, the sensitivity may be exacerbated by the PDR knockouts in the strain of S. cerevisiae that was used, and the associated growth inhibition was not strong.

Compound 4 also possessed moderate-strong anticancer activity against the HCT-116 cell line, with $\mathrm{IC}_{50}$ of $1.91 \mu \mathrm{M}$. However mithramycin A was far more potent, with an $\mathrm{IC}_{50}$ of 
0.017, suggesting that if compound $\mathbf{5}$ were isolated in suitable quantities for bioactivity assays, it too may exhibit cytotoxic effects to a higher degree than compound $\mathbf{4}$.

\subsection{Discussion}

As described in this chapter, multiple overlapping cosmids spanning a complete type II PKS biosynthetic pathway were recovered from the Half Moon Bay metagenome library, and reassembled by transformation-associated recombination to reconstitute a complete biosynthetic gene cluster on a single contiguous genetic construct. The genetic contents and metabolic output of the resulting construct were studied, showing that the encoded metabolites were new members of the aureolic acid family. Heterologous expression of this pathway in $S$. albus resulted in the production of many pathway-associated metabolites with aureolic acid like profiles, and two of these compounds were characterised (compounds $\mathbf{4}$ and $\mathbf{5}$ ).

The molecular formula of the major metabolite (compound 4) was determined by HR-MS to be $\mathrm{C}_{48} \mathrm{H}_{64} \mathrm{O}_{21}$, which is identical to a known aureolic acid, chromocyclomycin. Later isolation of compound $\mathbf{4}$ revealed that it was not chromocyclomycin, but instead a derivative structure where the glycosidic linkages were exclusively in the $\beta$ configuration; in contrast, in chromocyclomycin two mycarose sugars are $\alpha$-linked. Chromocyclomycin has only been described in three research publications, where the biological activity is either not described ${ }^{187,204}$ or it is stated to be "biologically inactive" ${ }^{202}$. Another analogous compound, the tetracyclic aureolic acid precursor premithramycin $\mathrm{B}$, has also been demonstrated to possess no detectable antibiotic activity (no inhibition of Micrococcus luteus growth by $50 \mu \mathrm{g}$ in bioassay) ${ }^{206}$. However, compound 4 has here been demonstrated to possess Gram-positive antibiotic activity comparable to that of tetracycline (Table 6.3). Likewise, compound 4 possessed moderate-strong anticancer cytotoxicity in the HCT-116 cell line. These results indicate that compound $\mathbf{4}$ possesses not only a novel chemical structure, but also a novel bioactivity profile.

In other known aureolic acids (with the exception of chromocyclomycin), glycosylated tetracyclic intermediates are metabolised by oxidative cleavage of the fourth ring to the tricyclic core found in the final bioactive compound ${ }^{48,206}$. Through bioactivity guided fractionation, a metabolite with a molecular formula matching that of a "ring-opened" metabolite of compound $\mathbf{4}$ was detected in the culture extract (compound 5). The tricyclic 
structure of this compound, with accompanying aliphatic tail, and mono and tri-saccharide deoxy sugar chains, was detected in the MS/MS spectrum (Figure 6.9). By overexpressing the genes responsible for catalysing this transformation, the production of the "ringopened" metabolite almost doubled in fermentation (Figure 6.10). While this increased production rate was a confirmation of the enzyme catalysed metabolic conversion, it was insufficient to permit full compound isolation. The bioactivity guided fractionation did however indicate a significant associated Gram-positive antibacterial activity, which is associated with the "ring-opening" reaction in other aureolic acid pathways (i.e. premithramycin $\mathrm{B} \rightarrow$ mithramycin) ${ }^{206}$. The linking of these two compounds by a specific biochemical transformation was also supporting evidence for determining the compound $\mathbf{5}$ structure based on fragmentation data and the structure of compound $\mathbf{4}$ as elucidated by 1D and 2D NMR experiments.

The gene content of the biosynthetic pathway is consistent with the two previously characterised aureolic acid biosynthesis schemes - mithramycin and chromomycin $\mathrm{A}_{3}-$ and matches the observed metabolic output (Figure 6.8). The biosynthetic pathway was predicted to encode enzymes with a relatively low average homology to those in mithramycin/chromomycin $\mathrm{A}_{3}$ biosynthesis, however the observations here indicate they encode similar biochemical functions. The major divergence between the biosynthetic output of mithramycin and the D3_2 biosynthesis pathway is in the disaccharide chain of mithramycin (D-olivose, D-oliose) where a single D-mycarose is substituted in compounds 4 and 5. In mithramycin these sugar additions are catalysed by MtmGI and MtmGII ${ }^{207}$, which are the two glycosyltransferases that share the lowest amino acid identities between the D3_2 pathway and mithramycin (Table 6.1 - GI 57.1\%, GII 58.4\% amino acid homology). The low amino acid identities likely explain the divergence in substrate specificity of these enzymes, and therefore the final pathway metabolic output.

With the continued hunt for new aureolic acids and derivatives (mithralogs), the discovery a new biosynthetic pathway and novel chemical structures is a relevant and useful contribution to the literature. This described biosynthetic gene cluster is only the third pathway characterised for aureolic acid production, and the two characterised metabolites are both of novel structure, and seemingly both active Gram-positive antibiotics. It is likely, on the weight of the presented evidence that if compound $\mathbf{5}$ were isolated in sufficient quantities to permit testing, it would also possess potent anticancer activity. 


\subsection{Summary, conclusions and future directions}

\subsection{Research motivation}

The study of natural product biosynthesis is of great scientific interest not only as it relates to intriguing genetic arrangements, biosynthetic pathways and chemical transformations, but also for the potential of medical impact. In the "race against time" to develop new antibiotics to combat widespread antibiotic resistance, as described by the World Health Organisation ${ }^{208}$, primary research into bacterial natural products is more important than ever. With the growing research interest into bacterial natural products, it is important to take a non-biased evaluative approach, to avoid simply applying more expensive tools and processes to the same workflows and arriving at the same outcomes.

The aim of this research was to apply an exploratory methodology to evaluate the biosynthetic potential of New Zealand soil microbiomes, and discover new bioactive compounds from this previously unexplored resource. This commenced with a large-scale functional screen in which cosmid metagenome libraries were constructed, and enriched them for secondary metabolism genes. The aim of this was primarily to establish a renewable "sequence bank" of biosynthetic gene cluster sequences, however it soon became apparent that an investigation of this scale could be used to provide global insights into secondary metabolic potential of metagenomes and non-cultivated bacteria.

I also employed sequence based screening to target biosynthetic gene clusters encoding aromatic polyketides and use heterologous expression platforms to evaluate the metabolites they might produce. Two gene clusters from this methodology were investigated, leading to the discovery of not only novel genetic pathways, but in each case new chemistry.

\subsection{Key findings}

\subsubsection{Sequence first biosynthetic survey}

The first stage of this research was the extraction of environmental DNA, and the construction of cosmid metagenome libraries. Large insert libraries of the scale constructed in the course of this research are not common, due to the labour involved in DNA isolation, purification and cloning. 
Using these libraries for a functional screening campaign, I was able to recover a large number of megasynth(et)ase biosynthetic gene clusters - 427 NRPS, PKS or hybrid gene clusters, and 503 clusters total. To my knowledge the current most extensive biosynthetic survey in the literature is Crits-Cristoph et al. 2018, which found 326 complete and partial NRPS, PKS and hybrid gene clusters ${ }^{16}$. The clusters reported in Chapter 4 of this thesis were novel by many metrics - with low percentage amino acid identity to known biosynthesis enzymes (below 40\% mean and median identity in each library), many gene clusters linked to phyla underrepresented in biosynthetic sequence databases (and often sequence databases in general), and also novel domain and gene arrangements.

By employing the recently developed biosynthetic gene cluster comparison tools BiGSCAPE and CORASON, I was able to identify families of gene clusters that were common in my metagenome data, but were not represented in any cultivated strains. Two major conclusions can be drawn from this. First, that metagenomic analysis techniques are able to access biosynthetic diversity that has eluded culture-based discovery efforts. And second, the abundance of these patterns across the different soil metagenomes indicates that these pathways are evolutionarily successful in the soil microbiome, which may link to specific bioactive functions of ecological/medical relevance.

\subsubsection{Gene cluster and metabolites of the D9 cosmid}

The remainder of the research in this thesis was based on cosmids recovered from the Half Moon Bay metagenome library using a sequence-based screen targeted toward aromatic polyketides. One of the cosmids recovered produced a large amount of pathway-associated metabolites by heterologous expression in S. albus, with extracts indicating antibiotic activity. However, gene cluster sequence analysis indicated the pathway was truncated. Three of the pathway metabolites were identified for analysis (compounds 1, 2, and 3). After many attempted extraction and purification techniques, only compounds $\mathbf{1}$ and $\mathbf{2}$ could be isolated in sufficient quantities for further analysis. However, stability issues with compound 2 made further study of this compound intractable.

The structure of compound 1 was elucidated using HR-MS, 1D and 2D NMR experiments. Compound $\mathbf{1}$ is a new chemical entity however, bioactivity assays of this compound did not indicate antibacterial function consistent with that previously observed in culture extracts, indicating other pathway-associated metabolites (possibly compound 2) were 
responsible for that activity. Nonetheless, compound $\mathbf{1}$ did possess weak general antibacterial activity, and conformed to Lipinski's rules for drug-likeness.

The structure of compound $\mathbf{1}$ appears to be a substructure in another antibiotic produced by a type II PKS system - setomimycin. Analysis of the biosynthetic gene clusters responsible for the production of both compound $\mathbf{1}$ and setomimycin indicated some similarities of gene function, however average amino acid homology was low (68.1\%), and these functions are common throughout type II PKS biosynthetic pathways. Therefore, even though the compounds appear structurally related, their gene clusters do not appear to share a close evolutionary relationship.

\subsubsection{Novel aureolic acid gene cluster and metabolites}

Another cosmid recovered from the Half Moon Bay metagenome library contained a partial biosynthetic gene cluster with similarities to the aureolic acid family. This was clearly a fragmented gene cluster, which lacked drug resistance or drug efflux genes. In order to reconstruct the complete pathway, overlapping cosmid clones were recovered from the metagenome library, and the pathway was reconstruction via transformation associated recombination in yeast. The resulting reconstituted biosynthetic gene cluster had similarities to the two known aureolic acid biosynthesis systems, however with some key differences in gene content and arrangement, and an overall low homology of gene products (60.7\%, and $52.7 \%$ average amino acid identity to mithramycin and chromomycin $\mathrm{A}_{3}$ respectively).

Heterologous expression studies of the reconstituted pathway led to the discovery of two new aureolic acid compounds (compound 4 and 5) using $S$. albus as a host. Compound 4 was similar in structure to the previously discovered aureolic acid, chromocyclomycin, with key differences in the stereochemistry of the glycosidic linkages of one D-mycarose to the polyketide core, and the other D-mycarose to the D-oliose sugar.

Compound 5 was discovered by bioactivity-guided fractionation of the fermentation extract, and was detected by MS analysis. MS/MS experiments were used to propose a structure consistent with the biosynthetic gene cluster, observed bioactivity, and comparison to fragmentation patterns observed for a known reference standard (mithramycin). Further confirmation was then established by overexpression of enzymes 
responsible for conversion of compound $\mathbf{4}$ to compound $\mathbf{5}$, linking the observed structure to the genetic and biosynthetic mechanisms with a resulting increase in metabolite production.

Beyond the bioactivity guided fractionation studies (which indicated potent Gram-positive bioactivity associated with compound $\mathbf{5}$, typical of aureolic acid compounds), compound $\mathbf{5}$ could not be isolated at sufficient quantities to perform in depth, quantitative bioactivity assays. Compound 4 was assayed however, and possessed moderate Gram-positive antibiotic activity and antitumor activity, beyond that previously reported for either chromocyclomycin or other "precursor" aureolic acids.

\subsection{Future directions}

\subsubsection{Biosynthetic gene clusters from functional screening}

The obvious next step for the biosynthetic gene clusters recovered in the PPTase functional screening study would be to heterologously express these pathways, and characterise the resulting metabolites. This will be easier for some clusters than others. The gene clusters which were taxonomically classified with the Actinobacteria ( 7\%) would be candidates for expression in S. albus systems (as was performed in Chapters 5 and 6), or in other well characterised Streptomyces expression hosts: S. coelicolor ${ }^{73,74}$, avermitilis ${ }^{75}$, or lividans 76. The Proteobacterial gene clusters ( 40\%) would be candidates for expression in a range of hosts facilitated by the pJWC expression platform within Agrobacterium tumefaciens, Burkholderia graminis, Caulobacter vibrioides, E. coli, Pseudomonas putida, and Ralstonia metallidurans ${ }^{93,94}$.

The largest remaining constituency are the Acidobacterial gene clusters $(\sim 30 \%)$. These are potentially the most interesting candidates, as Acidobacteria have long been known to comprise a large proportion of the soil microbiome ${ }^{163,169,209}$, though very little is known about their secondary metabolism ${ }^{210}$. As there are few cultivated examples of Acidobacteria, and no reliable heterologous expression platform, the first step would be to attempt expression within other Gram-negative hosts - using the same pJWC strategy as for the Proteobacteria gene clusters. There is precedence within this study for expression within Proteobacteria - the PPTase selection strategy relied on heterologous expression within the E. coli $\triangle e n t D$ screening strain. This will be explored as part of the doctoral 
research of Vincent Nowak, who will be investigating Acidobacteria secondary metabolism and will attempt to express some of these clusters in his studies.

Particular focus should be made on the gene cluster families identified by the BiG-SCAPE analysis in section 4.7. Representation of these families was common across multiple soil environments, and yet each gene cluster family was highly divergent to previously described biosynthesis. That these gene clusters are widely distributed indicates a particular evolutionary selection, which might indicate interesting bioactivity.

\subsubsection{D9 cluster metabolites}

The D9 cosmid contains truncated secondary metabolism genes at the borders of its insert region, indicating that the pathway is incomplete. Overlapping cosmid clones for the D9 cosmid were not recovered in the course of this study, therefore finding these overlapping clones and completing the pathway should be the next step. If there are more biosynthesis genes required to complete the pathway, it is likely those biosynthetic transformations will result in new metabolites to be recovered, with more stability and possibly more potency of bioactivity.

Although there was not any convincing evidence of genetic relationship between the D9 gene cluster and that of setomimycin, it is possible the structure of compound $\mathbf{1}$ may be a substrate for SetI catalysed dimerisation. The setI gene could be synthesised and coexpressed in S. albus in a pIJ10257 expression system (as was performed in Chapter 6 for OIV/W). This could be in effect a "combinatorial biosynthesis" experiment, which may result in the production of setomimycin, or a related metabolite.

Further combinatorial biosynthesis experiments could be undertaken to decorate the core structure of compound 1. Many tailoring enzymes in type II PKS biosynthesis are promiscuous, and co-expression of a library of tailoring functions alongside a core polyketide could be an avenue to producing novel "unnatural" natural products ${ }^{211}$.

It is possible compound $\mathbf{1}$ may have bioactivities not observed in the relatively simple bioassay panel applied in this study. As the compound can be relatively easily produced and purified in high quantities, it may be worthwhile to undertake a more extensive set of experiments. Specific molecular targets (e.g. a kinase inhibitor panel) may be more illuminating than exclusively relying on cell growth assays. 
Our medicinal chemistry collaborators may be able to use results of such molecular target assays for further development of the compound structure. The described structure satisfies Lipinski's "rule of five" for drug-likeness without violation, and has some associated weak bioactivity, therefore may be a candidate for further development.

\subsubsection{Aureolic acid biosynthetic gene cluster}

One major unachieved goal of Chapter 6 was the recovery of sufficient quantities of compound 5, both for NMR analysis and quantifiable bioactivity testing. Expression of compound 5 was demonstrated to be almost doubled by overexpression of the OIV and W genes. It is unlikely that further modulation of gene expression could yield significantly more compound production. Possibly, by scaling up the fermentation to $>50 \mathrm{~L}$, sufficient compound 5 could be isolated to enable these experiments.

The metagenome aureolic acid biosynthetic gene cluster contains three regulatory elements. RI is a Streptomyces antibiotic regulatory protein (SARP), while RII and RIII are PadR and TetR family transcriptional regulators respectively. In native $S$. argillaceus mithramycin biosynthesis, overexpression of RI (MtmR) resulted in a 16-fold increase in mithramycin production ${ }^{183}$, and overexpression of RII (MtrY) resulted in a $47 \%$ increase in mithramycin production ${ }^{212}$. Counter to this, insertional inactivation of RII (CmmRII) in native $S$. griseus subsp. griseus chromomycin $\mathrm{A}_{3}$ biosynthesis was found to increase metabolite production by $70 \%{ }^{198}$. Neither mithramycin nor chromomycin $\mathrm{A}_{3}$ have a homologue to RIII. Replicating this overexpression/inactivation strategy in the metagenome aureolic acid gene cluster may have similar results, permitting increased production of compound $\mathbf{5}$.

In 2018, an engineered strain of S. lividans TK24 was reported to be capable of producing mithramycin A "with yields close of $3 \mathrm{~g} / \mathrm{L}$ " ${ }^{76}$. If the engineered strain were acquired and found to be capable of expressing the metagenome aureolic acid gene cluster to levels even close to that of the mithramycin pathway, production problems would be solved.

If the large-scale production of compound $\mathbf{5}$ could be achieved, generation of derivatives based on previously successful strategies for mithramycin and chromomycin $\mathrm{A}_{3}$ could be attempted. In each case knockouts of the sidechain ketoreductase ( $\triangle \mathrm{mtm} W, \Delta \mathrm{cmmWI})$ resulted in derivatives with increased antitumor activity, and reduced off target toxicity, or 
both ${ }^{48,193,197}$. As the biosynthesis schemes are very similar, it is likely this derivatisation strategy would produce analogous compounds. Such a panel could then be tested against a wider range of cancer cell lines, to investigate any differences in activity relative to the mithramycin/chromomycin $\mathrm{A}_{3}$ derivatives.

The observed bioactivity of compound $\mathbf{4}$ may also deserve further exploration. Compound 4 was moderately active as both a Gram-positive antibiotic, and anticancer agent. These activities are not associated with either chromocyclomycin, or premithramycin B (the two closest compound structures) ${ }^{202,206}$. If these activities can be explored without the associated toxicity issues found with the other aureolic acids, this may be a lead worth exploring for drug development.

\subsection{Concluding remarks}

This thesis describes a series of investigations in the secondary metabolism of bacteria in New Zealand soil microbiomes. By first generating four large metagenome libraries, and then applying a large-scale functional screening and DNA sequencing program, new insights were gained into both abundance of biosynthetic gene clusters in our environment, and specific modes of biosynthesis which had not previously been observed. This will likely form the basis of future work into natural product drug discovery from soil bacteria and metagenomes. The gene clusters used for heterologous expression studies each resulted in the discovery of new metabolites. For the aureolic acid pathway, analytical chemistry and synthetic biology approaches were used in concert to associate gene cluster feature to metabolite structure and biosynthetic transformations. Both of the biosynthetic pathways studied here present opportunities for additional exploration and discovery of new bioactive metabolites. A similar pathway of investigation into the underrepresented clades of secondary metabolism identified in this thesis will likely lead to future discoveries. 


\section{References}

1. Cragg, G. M. \& Newman, D. J. Natural products: A continuing source of novel drug leads. Biochim. Biophys. Acta - Gen. Subj. 1830, 3670-3695 (2013).

2. Newman, D. J. \& Cragg, G. M. Natural Products as Sources of New Drugs from 1981 to 2014. J. Nat. Prod. 79, 629-661 (2016).

3. Lewis, K. Antibiotics: Recover the lost art of drug discovery. Nature 485, 439-440 (2012).

4. Lyddiard, D., Jones, G. L. \& Greatrex, B. W. Keeping it simple: Lessons from the golden era of antibiotic discovery. FEMS Microbiol. Lett. 363, fnw084 (2016).

5. Baltz, R. H. Natural product drug discovery in the genomic era: realities, conjectures, misconceptions, and opportunities. J. Ind. Microbiol. Biotechnol. 46, 281-299 (2019).

6. Katz, L. \& Baltz, R. H. Natural product discovery: past, present, and future. J. Ind. Microbiol. Biotechnol. 43, 155-176 (2016).

7. Torsvik, V., Goksoyr, J. \& Daae, F. L. High diversity in DNA of soil bacteria. Appl. Environ. Microbiol. 56, 782-787 (1990).

8. Torsvik, V., Daae, F. L., Sandaa, R. A. \& Øvreås, L. Novel techniques for analysing microbial diversity in natural and perturbed environments. J. Biotechnol. 64, 53-62 (1998).

9. Rappé, M. S. \& Giovannoni, S. J. The Uncultured Microbial Majority. Annu. Rev. Microbiol. 57, 369-394 (2003).

10. Daniel, R. The metagenomics of soil. Nat. Rev. Microbiol. 3, 470-478 (2005).

11. Bentley, S. D., Chater, K. F., Cerdeño-Tárraga, A.-M., Challis, G. L., Thomson, N. R., James, K. D., Harris, D. E., Quail, M. A., Kieser, H., Harper, D., Bateman, A., Brown, S., Chandra, G., Chen, C. W., Collins, M., Cronin, A., Fraser, A., Goble, A., Hidalgo, J., Hornsby, T., Howarth, S., Huang, C.-H., Kieser, T., Larke, L., Murphy, L., Oliver, K., O’Neil, S., Rabbinowitsch, E., Rajandream, M.-A., Rutherford, K., Rutter, S., Seeger, K., Saunders, D., Sharp, S., Squares, R., Squares, S., Taylor, K., Warren, T., Wietzorrek, A., Woodward, J., Barrell, B. G., Parkhill, J. \& Hopwood, 
D. A. Complete genome sequence of the model actinomycete Streptomyces coelicolor A3(2). Nature 417, 141-7 (2002).

12. Abrudan, M. I., Smakman, F., Grimbergen, A. J., Westhoff, S., Miller, E. L., Van Wezel, G. P. \& Rozen, D. E. Socially mediated induction and suppression of antibiosis during bacterial coexistence. Proc. Natl. Acad. Sci. U. S. A. 112, 11054 11059 (2015).

13. Mao, D., Okada, B. K., Wu, Y., Xu, F. \& Seyedsayamdost, M. R. Recent advances in activating silent biosynthetic gene clusters in bacteria. Curr. Opin. Microbiol. 45, 156-163 (2018).

14. Charlop-Powers, Z., Owen, J. G., Reddy, B. V. B., Ternei, M. A. \& Brady, S. F. Chemical-biogeographic survey of secondary metabolism in soil. Proc. Natl. Acad. Sci. 111, 3757-3762 (2014).

15. Charlop-Powers, Z., Owen, J. G., Reddy, B. V. B., Ternei, M., Guimaraes, D. O., De Frias, U. A., Pupo, M. T., Seepe, P., Feng, Z. \& Brady, S. F. Global biogeographic sampling of bacterial secondary metabolism. Elife 2015, e05048 (2015).

16. Crits-Christoph, A., Diamond, S., Butterfield, C. N., Thomas, B. C. \& Banfield, J. F. Novel soil bacteria possess diverse genes for secondary metabolite biosynthesis. Nature 558, 440-444 (2018).

17. Walsh, C. T. The chemical versatility of natural-product assembly lines. Acc. Chem. Res. 41, 4-10 (2008).

18. Hertweck, C. The biosynthetic logic of polyketide diversity. Angew. Chemie - Int. Ed. 48, 4688-4716 (2009).

19. Walsh, C. T. Insights into the chemical logic and enzymatic machinery of NRPS assembly lines. Nat. Prod. Rep. 33, 127-135 (2016).

20. Izoré, T. \& Cryle, M. J. The many faces and important roles of protein-protein interactions during non-ribosomal peptide synthesis. Nat. Prod. Rep. 35, 1120-1139 (2018).

21. Medema, M. H., Kottmann, R., Yilmaz, P., Cummings, M., Biggins, J. B., Blin, K., De Bruijn, I., Chooi, Y. H., Claesen, J., Coates, R. C., Cruz-Morales, P., Duddela, 
S., Düsterhus, S., Edwards, D. J., Fewer, D. P., Garg, N., Geiger, C., GomezEscribano, J. P., Greule, A., Hadjithomas, M., Haines, A. S., Helfrich, E. J. N., Hillwig, M. L., Ishida, K., Jones, A. C., Jones, C. S., Jungmann, K., Kegler, C., Kim, H. U., Kötter, P., Krug, D., Masschelein, J., Melnik, A. V, Mantovani, S. M., Monroe, E. A., Moore, M., Moss, N., Nützmann, H. W., Pan, G., Pati, A., Petras, D., Reen, F. J., Rosconi, F., Rui, Z., Tian, Z., Tobias, N. J., Tsunematsu, Y., Wiemann, P., Wyckoff, E., Yan, X., Yim, G., Yu, F., Xie, Y., Aigle, B., Apel, A. K., Balibar, C. J., Balskus, E. P., Barona-Gómez, F., Bechthold, A., Bode, H. B., Borriss, R., Brady, S. F., Brakhage, A. A., Caffrey, P., Cheng, Y. Q., Clardy, J., Cox, R. J., De Mot, R., Donadio, S., Donia, M. S., Van Der Donk, W. A., Dorrestein, P. C., Doyle, S., Driessen, A. J. M., Ehling-Schulz, M., Entian, K. D., Fischbach, M. A., Gerwick, L., Gerwick, W. H., Gross, H., Gust, B., Hertweck, C., Höfte, M., Jensen, S. E., Ju, J., Katz, L., Kaysser, L., Klassen, J. L., Keller, N. P., Kormanec, J., Kuipers, O. P., Kuzuyama, T., Kyrpides, N. C., Kwon, H. J., Lautru, S., Lavigne, R., Lee, C. Y., Linquan, B., Liu, X., Liu, W., Luzhetskyy, A., Mahmud, T., Mast, Y., Méndez, C., Metsä-Ketelä, M., Micklefield, J., Mitchell, D. A., Moore, B. S., Moreira, L. M., Müller, R., Neilan, B. A., Nett, M., Nielsen, J., O’Gara, F., Oikawa, H., Osbourn, A., Osburne, M. S., Ostash, B., Payne, S. M., Pernodet, J. L., Petricek, M., Piel, J., Ploux, O., Raaijmakers, J. M., Salas, J. A., Schmitt, E. K., Scott, B., Seipke, R. F., Shen, B., Sherman, D. H., Sivonen, K., Smanski, M. J., Sosio, M., Stegmann, E., Süssmuth, R. D., Tahlan, K., Thomas, C. M., Tang, Y., Truman, A. W., Viaud, M., Walton, J. D., Walsh, C. T., Weber, T., Van Wezel, G. P., Wilkinson, B., Willey, J. M., Wohlleben, W., Wright, G. D., Ziemert, N., Zhang, C., Zotchev, S. B., Breitling, R., Takano, E. \& Glöckner, F. O. Minimum Information about a Biosynthetic Gene cluster. Nat. Chem. Biol. 11, 625-631 (2015).

22. Blin, K., Wolf, T., Chevrette, M. G., Lu, X., Schwalen, C. J., Kautsar, S. A., Suarez Duran, H. G., De Los Santos, E. L. C., Kim, H. U., Nave, M., Dickschat, J. S., Mitchell, D. A., Shelest, E., Breitling, R., Takano, E., Lee, S. Y., Weber, T. \& Medema, M. H. AntiSMASH 4.0 - improvements in chemistry prediction and gene cluster boundary identification. Nucleic Acids Res. 45, W36-W41 (2017).

23. Skinnider, M. A., Merwin, N. J., Johnston, C. W. \& Magarvey, N. A. PRISM 3: Expanded prediction of natural product chemical structures from microbial genomes. Nucleic Acids Res. 45, W49-W54 (2017). 
24. Dejong, C. A., Chen, G. M., Li, H., Johnston, C. W., Edwards, M. R., Rees, P. N., Skinnider, M. A., Webster, A. L. H. \& Magarvey, N. A. Polyketide and nonribosomal peptide retro-biosynthesis and global gene cluster matching. Nat. Chem. Biol. 12, 1007-1014 (2016).

25. Ricart, E., Leclère, V., Flissi, A., Mueller, M., Pupin, M. \& Lisacek, F. RBAN: Retro-biosynthetic analysis of nonribosomal peptides. J. Cheminform. 11, 13 (2019).

26. Ikeda, H., Ishikawa, J., Hanamoto, A., Shinose, M., Kikuchi, H., Shiba, T., Sakaki, Y., Hattori, M. \& Omura, S. Complete genome sequence and comparative analysis of the industrial microorganism Streptomyces avermitilis. Nat. Biotechnol. 21, 526$531(2003)$.

27. Oliynyk, M., Samborskyy, M., Lester, J. B., Mironenko, T., Scott, N., Dickens, S., Haydock, S. F. \& Leadlay, P. F. Complete genome sequence of the erythromycinproducing bacterium Saccharopolyspora erythraea NRRL23338. Nat. Biotechnol. 25, 447-453 (2007).

28. Wang, X. J., Yan, Y. J., Zhang, B., An, J., Wang, J. J., Tian, J., Jiang, L., Chen, Y. H., Huang, S. X., Yin, M., Zhang, J., Gao, A. L., Liu, C. X., Zhu, Z. X. \& Xiang, W. S. Genome sequence of the milbemycin-producing bacterium Streptomyces bingchenggensis. J. Bacteriol. 192, 4526-4527 (2010).

29. Aigle, B., Lautru, S., Spiteller, D., Dickschat, J. S., Challis, G. L., Leblond, P. \& Pernodet, J. L. Genome mining of Streptomyces ambofaciens. J. Ind. Microbiol. Biotechnol. 41, 251-263 (2014).

30. Stevenson, L. J., Owen, J. G. \& Ackerley, D. F. Metagenome Driven Discovery of Nonribosomal Peptides. ACS Chem. Biol. 14, 2115-2126 (2019).

31. Walsh, C. T., O’Brien, R. V. \& Khosla, C. Nonproteinogenic amino acid building blocks for nonribosomal peptide and hybrid polyketide scaffolds. Angew. Chemie Int. Ed. 52, 7098-7124 (2013).

32. Walsh, C. T., Chen, H., Keating, T. A., Hubbard, B. K., Losey, H. C., Luo, L., Marshall, C. G., Miller, D. A. \& Patel, H. M. Tailoring enzymes that modify nonribosomal peptides during and after chain elongation on NRPS assembly lines. Curr. Opin. Chem. Biol. 5, 525-534 (2001). 
33. Brown, A. S., Calcott, M. J., Owen, J. G. \& Ackerley, D. F. Structural, functional and evolutionary perspectives on effective re-engineering of non-ribosomal peptide synthetase assembly lines. Nat. Prod. Rep. 35, 1210-1228 (2018).

34. Stachelhaus, T. \& Marahiel, M. A. Modular structure of genes encoding multifunctional peptide synthetases required for non-ribosomal peptide synthesis. FEMS Microbiol. Lett. 125, 3-14 (1995).

35. Kleinkauf, H. \& Von Döhren, H. A nonribosomal system of peptide biosynthesis. Eur. J. Biochem. 236, 335-351 (1996).

36. Baltz, R. H. Function of MbtH homologs in nonribosomal peptide biosynthesis and applications in secondary metabolite discovery. J. Ind. Microbiol. Biotechnol. 38, 1747-1760 (2011).

37. Fischbach, M. A. \& Walsh, C. T. Assembly-Line Enzymology for Polyketide and Nonribosomal Peptide Antibiotics: Logic, Machinery, and Mechanisms. Chem. Rev. 106, 3468-3496 (2006).

38. Scott, T. A. \& Piel, J. The 'hidden' enzymology of natural product biosynthesis Thomas. Nat. Rev. Chem. 1, 169-174 (2016).

39. Helfrich, E. J. N. \& Piel, J. Biosynthesis of polyketides by trans-AT polyketide synthases. Nat. Prod. Rep. 33, 231-316 (2016).

40. Yadav, G., Gokhale, R. S. \& Mohanty, D. Computational approach for prediction of domain organization and substrate specificity of modular polyketide synthases. $J$. Mol. Biol. 328, 335-363 (2003).

41. Chen, H., O’Connor, S., Cane, D. E. \& Walsh, C. T. Epothilone biosynthesis: Assembly of the methylthiazolylcarboxy starter unit on the EpoB subunit. Chem. Biol. 8, 899-912 (2001).

42. Hertweck, C., Luzhetskyy, A., Rebets, Y. \& Bechthold, A. Type II polyketide synthases: Gaining a deeper insight into enzymatic teamwork. Nat. Prod. Rep. 24, 162-190 (2007).

43. Kharel, M. K., Pahari, P., Shepherd, M. D., Tibrewal, N., Nybo, S. E., Shaaban, K. A. \& Rohr, J. Angucyclines: Biosynthesis, mode-of-action, new natural products, and synthesis. Nat. Prod. Rep. 29, 264-325 (2012). 
44. Singh, G. M., Fortin, P. D., Koglin, A. \& Walsh, C. T. $\beta$-hydroxylation of the aspartyl residue in the phytotoxin syringomycin E: Characterization of two candidate hydroxylases AspH and SyrP in Pseudomonas syringae. Biochemistry 47, 1131011320 (2008).

45. Ciabatti, R., Kettenring, J. K., Winters, G., Tuan, G., Zerilli, L. \& Cavalleri, B. Ramoplanin (A-16686), a new glycolipodepsipeptide antibiotic. III. Structure elucidation. J. Antibiot. (Tokyo). 42, 254-267 (2012).

46. Křen, V. \& Řezanka, T. Sweet antibiotics - The role of glycosidic residues in antibiotic and antitumor activity and their randomization. FEMS Microbiol. Rev. 32, $858-889$ (2008).

47. Lombó, F., Olano, C., Salas, J. A. \& Méndez, C. Chapter 11 Sugar Biosynthesis and Modification. in Methods in Enzymology 458, 277-308 (Academic Press, 2009).

48. Menéndez, N., Nur-e-Alam, M., Braña, A. F., Rohr, J., Salas, J. A. \& Méndez, C. Biosynthesis of the Antitumor Chromomycin A3 in Streptomyces griseus. Chem. Biol. 11, 21-32 (2009).

49. Lombó, F., Menéndez, N., Salas, J. A. \& Méndez, C. The aureolic acid family of antitumor compounds: Structure, mode of action, biosynthesis, and novel derivatives. Appl. Microbiol. Biotechnol. 73, 1-14 (2006).

50. Lazos, O., Tosin, M., Slusarczyk, A. L., Boakes, S., Cortés, J., Sidebottom, P. J. \& Leadlay, P. F. Biosynthesis of the Putative Siderophore Erythrochelin Requires Unprecedented Crosstalk between Separate Nonribosomal Peptide Gene Clusters. Chem. Biol. 17, 160-173 (2010).

51. García, B., González-Sabín, J., Menéndez, N., Brana, A. F., Nunez, L. E., Morís, F., Salas, J. A. \& Méndez, C. The chromomycin CmmA acetyltransferase: A membrane-bound enzyme as a tool for increasing structural diversity of the antitumour mithramycin. Microb. Biotechnol. 4, 226-238 (2011).

52. Feng, Z., Qi, J., Tsuge, T., Oba, Y., Kobayashi, T., Suzuki, Y., Sakagami, Y. \& Ojika, M. Construction of a Bacterial Artificial Chromosome Library for a Myxobacterium of the Genus Cystobacter and Characterization of an Antibiotic Biosynthetic Gene Cluster. Biosci. Biotechnol. Biochem. 69, 1372-1380 (2005). 
53. Buntin, K., Weissman, K. J. \& Müller, R. An unusual thioesterase promotes isochromanone ring formation in ajudazol biosynthesis. ChemBioChem 11, 1137$1146(2010)$.

54. Walsh, C. T. Nature Builds Macrocycles and Heterocycles into Its Antimicrobial Frameworks: Deciphering Biosynthetic Strategy. ACS Infect. Dis. 4, 1283-1299 (2018).

55. Stachelhaus, T., Mootz, H. D. \& Marahiel, M. A. The specificity-conferring code of adenylation domains in nonribosomal peptide synthetases. Chem. Biol. 6, 493-505 (1999).

56. Challis, G. L., Ravel, J. \& Townsend, C. A. Predictive, structure-based model of amino acid recognition by nonribosomal peptide synthetase adenylation domains. Chem. Biol. 7, 211-224 (2000).

57. Minowa, Y., Araki, M. \& Kanehisa, M. Comprehensive Analysis of Distinctive Polyketide and Nonribosomal Peptide Structural Motifs Encoded in Microbial Genomes. J. Mol. Biol. 368, 1500-1517 (2007).

58. Khayatt, B. I., Overmars, L., Siezen, R. J. \& Francke, C. Classification of the Adenylation and Acyl-Transferase Activity of NRPS and PKS Systems Using Ensembles of Substrate Specific Hidden Markov Models. PLoS One 8, e62136 (2013).

59. Rausch, C., Weber, T., Kohlbacher, O., Wohlleben, W. \& Huson, D. H. Specificity prediction of adenylation domains in nonribosomal peptide synthetases (NRPS) using transductive support vector machines (TSVMs). Nucleic Acids Res. 33, 57995808 (2005).

60. Röttig, M., Medema, M. H., Blin, K., Weber, T., Rausch, C. \& Kohlbacher, O. NRPSpredictor2 - A web server for predicting NRPS adenylation domain specificity. Nucleic Acids Res. 39, W362-W367 (2011).

61. Chevrette, M. G., Aicheler, F., Kohlbacher, O., Currie, C. R. \& Medema, M. H. SANDPUMA: Ensemble predictions of nonribosomal peptide chemistry reveal biosynthetic diversity across Actinobacteria. Bioinformatics 33, 3202-3210 (2017).

62. Yadav, G., Gokhale, R. S. \& Mohanty, D. SEARCHPKS: A program for detection 
and analysis of polyketide synthase domains. Nucleic Acids Res. 31, 3654-3658 (2003).

63. Medema, M. H., Blin, K., Cimermancic, P., De Jager, V., Zakrzewski, P., Fischbach, M. A., Weber, T., Takano, E. \& Breitling, R. AntiSMASH: Rapid identification, annotation and analysis of secondary metabolite biosynthesis gene clusters in bacterial and fungal genome sequences. Nucleic Acids Res. 39, W339-W346 (2011).

64. Blin, K., Medema, M. H., Kazempour, D., Fischbach, M. A., Breitling, R., Takano, E. \& Weber, T. antiSMASH 2.0--a versatile platform for genome mining of secondary metabolite producers. Nucleic Acids Res. 41, W204-W212 (2013).

65. Weber, T., Blin, K., Duddela, S., Krug, D., Kim, H. U., Bruccoleri, R., Lee, S. Y., Fischbach, M. A., Müller, R., Wohlleben, W., Breitling, R., Takano, E. \& Medema, M. H. AntiSMASH 3.0-A comprehensive resource for the genome mining of biosynthetic gene clusters. Nucleic Acids Res. 43, W237-W243 (2015).

66. Skinnider, M. A., Dejong, C. A., Rees, P. N., Johnston, C. W., Li, H., Webster, A. L. H., Wyatt, M. A. \& Magarvey, N. A. Genomes to natural products PRediction Informatics for Secondary Metabolomes (PRISM). Nucleic Acids Res. 43, 9645$9662(2015)$.

67. Lautru, S., Deeth, R. J., Bailey, L. M. \& Challis, G. L. Discovery of a new peptide natural product by streptomyces coelicolor genome mining. Nat. Chem. Biol. 1, 265$269(2005)$.

68. Gomez-Escribano, J. P., Song, L., Fox, D. J., Yeo, V., Bibb, M. J. \& Challis, G. L. Structure and biosynthesis of the unusual polyketide alkaloid coelimycin P1, a metabolic product of the cpk gene cluster of Streptomyces coelicolor M145. Chem. Sci. 3, 2716-2720 (2012).

69. Lucas, X., Senger, C., Erxleben, A., Grüning, B. A., Döring, K., Mosch, J., Flemming, S. \& Günther, S. StreptomeDB: A resource for natural compounds isolated from Streptomyces species. Nucleic Acids Res. 41, D1130-D1136 (2013).

70. Hoffmann, T., Krug, D., Bozkurt, N., Duddela, S., Jansen, R., Garcia, R., Gerth, K., Steinmetz, H. \& Müller, R. Correlating chemical diversity with taxonomic distance for discovery of natural products in myxobacteria. Nat. Commun. 9, 803 (2018). 
71. Dhakal, D., Rayamajhi, V., Mishra, R. \& Sohng, J. K. Bioactive molecules from Nocardia: diversity, bioactivities and biosynthesis. J. Ind. Microbiol. Biotechnol. 46, 385-407 (2019).

72. Chevrette, M. G. \& Currie, C. R. Emerging evolutionary paradigms in antibiotic discovery. J. Ind. Microbiol. Biotechnol. 46, 257-271 (2019).

73. Gomez-Escribano, J. P. \& Bibb, M. J. Engineering Streptomyces coelicolor for heterologous expression of secondary metabolite gene clusters. Microb. Biotechnol. 4, 207-215 (2011).

74. Thanapipatsiri, A., Claesen, J., Gomez-Escribano, J. P., Bibb, M. \& Thamchaipenet, A. A Streptomyces coelicolor host for the heterologous expression of Type III polyketide synthase genes. Microb. Cell Fact. 14, 145 (2015).

75. Komatsu, M., Komatsu, K., Koiwai, H., Yamada, Y., Kozone, I., Izumikawa, M., Hashimoto, J., Takagi, M., Omura, S., Shin-Ya, K., Cane, D. E. \& Ikeda, H. Engineered Streptomyces avermitilis host for heterologous expression of biosynthetic gene cluster for secondary metabolites. ACS Synth. Biol. 2, 384-396 (2013).

76. Novakova, R., Núñez, L. E., Homerova, D., Knirschova, R., Feckova, L., Rezuchova, B., Sevcikova, B., Menéndez, N., Morís, F., Cortés, J. \& Kormanec, J. Increased heterologous production of the antitumoral polyketide mithramycin A by engineered Streptomyces lividans TK24 strains. Appl. Microbiol. Biotechnol. 102, 857-869 (2018).

77. Myronovskyi, M., Rosenkränzer, B., Nadmid, S., Pujic, P., Normand, P. \& Luzhetskyy, A. Generation of a cluster-free Streptomyces albus chassis strains for improved heterologous expression of secondary metabolite clusters. Metab. Eng. 49, 316-324 (2018).

78. Owen, J. G., Reddy, B. V. B., Ternei, M. A., Charlop-Powers, Z., Calle, P. Y., Kim, J. H. \& Brady, S. F. Mapping gene clusters within arrayed metagenomic libraries to expand the structural diversity of biomedically relevant natural products. Proc. Natl. Acad. Sci. 110, 11797-11802 (2013).

79. Owen, J. G., Charlop-Powers, Z., Smith, A. G., Ternei, M. A., Calle, P. Y., Reddy, 
B. V. B., Montiel, D. \& Brady, S. F. Multiplexed metagenome mining using short DNA sequence tags facilitates targeted discovery of epoxyketone proteasome inhibitors. Proc. Natl. Acad. Sci. 112, 4221-4226 (2015).

80. Hover, B. M., Kim, S. H., Katz, M., Charlop-Powers, Z., Owen, J. G., Ternei, M. A., Maniko, J., Estrela, A. B., Molina, H., Park, S., Perlin, D. S. \& Brady, S. F. Culture-independent discovery of the malacidins as calcium-dependent antibiotics with activity against multidrug-resistant Gram-positive pathogens. Nat. Microbiol. 3, 415-422 (2018).

81. Wu, C., Shang, Z., Lemetre, C., Ternei, M. A. \& Brady, S. F. Cadasides, CalciumDependent Acidic Lipopeptides from the Soil Metagenome That Are Active against Multidrug-Resistant Bacteria. J. Am. Chem. Soc. 141, 3910-3919 (2019).

82. Feng, Z., Kallifidas, D. \& Brady, S. F. Functional analysis of environmental DNAderived type II polyketide synthases reveals structurally diverse secondary metabolites. Proc. Natl. Acad. Sci. U. S. A. 108, 12629-12634 (2011).

83. Reddy, B. V. B., Kallifidas, D., Kim, J. H., Charlop-Powers, Z., Feng, Z. \& Brady, S. F. Natural Product Biosynthetic Gene Diversity in Geographically Distinct Soil Microbiomes. Appl. Environ. Microbiol. 78, 3744-3752 (2012).

84. Seow, K. T., Meurer, G., Gerlitz, M., Wendt-Pienkowski, E., Hutchinson, C. R. \& Davies, J. A study of iterative type II polyketide synthases, using bacterial genes cloned from soil DNA: A means to access and use genes from uncultured microorganisms. J. Bacteriol. 179, 7360-7368 (1997).

85. Metsä-Ketelä, M., Salo, V., Halo, L., Hautala, A., Hakala, J., Mäntsälä, P. \& Ylihonko, K. An efficient approach for screening minimal PKS genes from Streptomyces. FEMS Microbiol. Lett. 180, 1-6 (1999).

86. King, R. W., Bauer, J. D. \& Brady, S. F. An environmental DNA-derived type II polyketide biosynthetic pathway encodes the biosynthesis of the pentacyclic polyketide erdacin. Angew. Chemie - Int. Ed. 48, 6257-6261 (2009).

87. Feng, Z., Chakraborty, D., Dewell, S. B., Reddy, B. V. B. \& Brady, S. F. Environmental DNA-encoded antibiotics fasamycins A and B inhibit FabF in type II fatty acid biosynthesis. J. Am. Chem. Soc. 134, 2981-2987 (2012). 
88. Lemetre, C., Maniko, J., Charlop-Powers, Z., Sparrow, B., Lowe, A. J. \& Brady, S. F. Bacterial natural product biosynthetic domain composition in soil correlates with changes in latitude on a continent-wide scale. Proc. Natl. Acad. Sci. 114, 1161511620 (2017).

89. Benaud, N., Zhang, E., Van Dorst, J., Brown, M. V, Kalaitzis, J. A., Neilan, B. A. \& Ferrari, B. C. Harnessing long-read amplicon sequencing to uncover NRPS and Type i PKS gene sequence diversity in polar desert soils. FEMS Microbiol. Ecol. 95, (2019).

90. Masschelein, J., Jenner, M. \& Challis, G. L. Antibiotics from Gram-negative bacteria: A comprehensive overview and selected biosynthetic highlights. Nat. Prod. Rep. 34, 712-783 (2017).

91. Zhang, J. J., Tang, X. \& Moore, B. S. Genetic platforms for heterologous expression of microbial natural products. Nat. Prod. Rep. (2019).

92. Brady, S. F. Construction of soil environmental DNA cosmid libraries and screening for clones that produce biologically active small molecules. Nat. Protoc. 2, 12971305 (2007).

93. Craig, J. W., Chang, F. Y. \& Brady, S. F. Natural products from environmental DNA hosted in Ralstonia metallidurans. ACS Chem. Biol. 4, 23-28 (2009).

94. Craig, J. W., Chang, F. Y., Kim, J. H., Obiajulu, S. C. \& Brady, S. F. Expanding small-molecule functional metagenomics through parallel screening of broad-hostrange cosmid environmental DNA libraries in diverse proteobacteria. Appl. Environ. Microbiol. 76, 1633-1641 (2010).

95. Iqbal, H. A., Low-Beinart, L., Obiajulu, J. U. \& Brady, S. F. Natural Product Discovery through Improved Functional Metagenomics in Streptomyces. J. Am. Chem. Soc. 138, 9341-9344 (2016).

96. Stevens, D. C., Conway, K. R., Pearce, N., Villegas-Peñaranda, L. R., Garza, A. G. \& Boddy, C. N. Alternative Sigma Factor Over-Expression Enables Heterologous Expression of a Type II Polyketide Biosynthetic Pathway in Escherichia coli. PLoS One 8, 7045 (2013).

97. Gaida, S. M., Sandoval, N. R., Nicolaou, S. A., Chen, Y., Venkataramanan, K. P. \& 
Papoutsakis, E. T. Expression of heterologous sigma factors enables functional screening of metagenomic and heterologous genomic libraries. Nat. Commun. 6, 7045 (2015).

98. Kallifidas, D., Kang, H. S. \& Brady, S. F. Tetarimycin A, an MRSA-active antibiotic identified through induced expression of environmental DNA gene clusters. J. Am. Chem. Soc. 134, 19552-19555 (2012).

99. Owen, J. G., Robins, K. J., Parachin, N. S. \& Ackerley, D. F. A functional screen for recovery of 4'-phosphopantetheinyl transferase and associated natural product biosynthesis genes from metagenome libraries. Environ. Microbiol. 14, 1198-1209 (2012).

100. Bitok, J. K., Lemetre, C., Ternei, M. A. \& Brady, S. F. Identification of biosynthetic gene clusters from metagenomic libraries using PPTase complementation in a Streptomyces host. FEMS Microbiol. Lett. 364, 233-237 (2017).

101. Charlop-Powers, Z., Banik, J. J., Owen, J. G., Craig, J. W. \& Brady, S. F. Selective enrichment of environmental DNA libraries for genes encoding nonribosomal peptides and polyketides by phosphopantetheine transferase- dependent complementation of siderophore biosynthesis. ACS Chem. Biol. 8, 138-143 (2013).

102. Simon, R., Priefer, U. \& Pühler, A. A broad host range mobilization system for in vivo genetic engineering: Transposon mutagenesis in gram negative bacteria. Bio/Technology 1, 784-791 (1983).

103. Gunther, E. J., Murray, N. E. \& Glazer, P. M. High efficiency, restriction-deficient in vitro packaging extracts for bacteriophage lambda DNA using a new E.coli lysogen. Nucleic Acids Res. 21, 3903-3904 (1993).

104. Hohn, B. In Vitro Packaging of $\lambda$ and Cosmid DNA. Methods Enzymol. 68, 299-309 (1979).

105. Copp, J. N., Williams, E. M., Rich, M. H., Patterson, A. V., Smaill, J. B., Ackerley, D. F. \& Ollis, D. Toward a high-throughput screening platform for directed evolution of enzymes that activate genotoxic prodrugs. Protein Eng. Des. Sel. 27, 399-403 (2014).

106. Chater, K. F. \& Wilde, L. C. Restriction of a bacteriophage of Streptomyces albus 
G involving endonuclease salI. J. Bacteriol. 128, 644-650 (1976).

107. Spizizen, J. Transformation of Biochemically Deficient Strains of Bacillus subtilis by Deoxyribonucleate. Proc. Natl. Acad. Sci. 44, 1072-1078 (1958).

108. Montiel, D., Kang, H. S., Chang, F. Y., Charlop-Powers, Z. \& Brady, S. F. Yeast homologous recombination-based promoter engineering for the activation of silent natural product biosynthetic gene clusters. Proc. Natl. Acad. Sci. U. S. A. 112, 89538958 (2015).

109. Coorey, N. V. C., Matthews, J. H., Bellows, D. S. \& Atkinson, P. H. Pleiotropic drug-resistance attenuated genomic library improves elucidation of drug mechanisms. Mol. Biosyst. 11, 3129-3136 (2015).

110. Bierman, M., Logan, R., O’Brien, K., Seno, E. T., Nagaraja Rao, R. \& Schoner, B. E. Plasmid cloning vectors for the conjugal transfer of DNA from Escherichia coli to Streptomyces spp. Gene 116, 43-49 (1992).

111. Kim, J. H., Feng, Z., Bauer, J. D., Kallifidas, D., Calle, P. Y. \& Brady, S. F. Cloning large natural product gene clusters from the environment: Piecing environmental DNA gene clusters back together with TAR. Biopolymers 93, 833-844 (2010).

112. Hong, H. J., Hutchings, M. I., Hill, L. M. \& Buttner, M. J. The role of the novel fem protein VanK in vancomycin resistance in Streptomyces coelicolor. J. Biol. Chem. 280, 13055-13061 (2005).

113. Winn, R. \& Norris, M. Analysis of mutations in $\lambda$ transgenic medaka using the cII mutation assay. in Techniques in Aquatic Toxicology, Volume 2 (CRC Press, 2010).

114. Kallifidas, D. \& Brady, S. F. Reassembly of functionally intact environmental DNAderived biosynthetic gene clusters. in Methods in Enzymology 517, 225-239 (Academic Press, 2012).

115. Wiegand, I., Hilpert, K. \& Hancock, R. E. W. Agar and broth dilution methods to determine the minimal inhibitory concentration (MIC) of antimicrobial substances. Nat. Protoc. 3, 163-175 (2008).

116. Buchfink, B., Xie, C. \& Huson, D. H. Fast and sensitive protein alignment using DIAMOND. Nat. Methods 12, 59-60 (2014). 
117. Navarro-Muñoz, J., Selem-Mojica, N., Mullowney, M., Kautsar, S., Tryon, J., Parkinson, E., Santos, E. D. L., Yeong, M., Cruz-Morales, P., Abubucker, S., Roeters, A., Lokhorst, W., Fernandez-Guerra, A., Cappelini, L. T. D., Thomson, R., Metcalf, W., Kelleher, N., Barona-Gomez, F. \& Medema, M. H. A computational framework for systematic exploration of biosynthetic diversity from large-scale genomic data. bioRxiv 445270 (2018).

118. medema-group / BiG-SCAPE - GitLab. Available at: https://git.wageningenur.nl/medema-group/BiG-SCAPE/. (Accessed: 8th July 2019)

119. Ghoul, M. \& Mitri, S. The Ecology and Evolution of Microbial Competition. Trends Microbiol. 24, 833-845 (2016).

120. Cornforth, D. M. \& Foster, K. R. Antibiotics and the art of bacterial war. Proc. Natl. Acad. Sci. 112, 10827-10828 (2015).

121. Waters, J. M. \& Craw, D. Goodbye Gondwana? New Zealand Biogeography, Geology, and the Problem of Circularity. Syst. Biol. 55, 351-356 (2006).

122. Wallis, G. P. \& Trewick, S. A. New Zealand phylogeography: Evolution on a small continent. Mol. Ecol. 18, 3548-3580 (2009).

123. Hewitt, A. E. \& Manaaki Whenua-Landcare Research New Zealand Ltd. New Zealand soil classification. (Manaaki Whenua Press, 2010).

124. diCenzo, G. C. \& Finan, T. M. The Divided Bacterial Genome: Structure, Function, and Evolution. Microbiol. Mol. Biol. Rev. 81, e00019-17 (2017).

125. Donadio, S., Monciardini, P. \& Sosio, M. Polyketide synthases and nonribosomal peptide synthetases: The emerging view from bacterial genomics. Nat. Prod. Rep. 24, 1073-1079 (2007).

126. Nett, M., Ikeda, H. \& Moore, B. S. Genomic basis for natural product biosynthetic diversity in the actinomycetes. Nat. Prod. Rep. 26, 1362-1384 (2009).

127. Hrvatin, S. \& Piel, J. Rapid isolation of rare clones from highly complex DNA libraries by PCR analysis of liquid gel pools. J. Microbiol. Methods 68, 434-436 (2007).

128. Beld, J., Sonnenschein, E. C., Vickery, C. R., Noel, J. P. \& Burkart, M. D. The 
phosphopantetheinyl transferases: Catalysis of a post-translational modification crucial for life. Nat. Prod. Rep. 31, 61-108 (2014).

129. Nurk, S., Bankevich, A., Antipov, D., Gurevich, A. A., Korobeynikov, A., Lapidus, A., Prjibelski, A. D., Pyshkin, A., Sirotkin, A., Sirotkin, Y., Stepanauskas, R., Clingenpeel, S. R., Woyke, T., Mclean, J. S., Lasken, R., Tesler, G., Alekseyev, M. A. \& Pevzner, P. A. Assembling Single-Cell Genomes and Mini-Metagenomes From Chimeric MDA Products. J. Comput. Biol. 20, 714-737 (2013).

130. Antipov, D., Korobeynikov, A., McLean, J. S. \& Pevzner, P. A. HybridSPAdes: An algorithm for hybrid assembly of short and long reads. Bioinformatics 32, 10091015 (2016).

131. Nurk, S., Meleshko, D., Korobeynikov, A. \& Pevzner, P. A. metaSPAdes: a new versatile metagenomic assembler. Genome Res. 27, 824-834 (2017).

132. Langmead, B. \& Salzberg, S. L. Fast gapped-read alignment with Bowtie 2. Nat. Methods 9, 357-9 (2012).

133. Bolger, A. M., Lohse, M. \& Usadel, B. Trimmomatic: a flexible trimmer for Illumina sequence data. Bioinformatics 30, 2114-2120 (2014).

134. Shen, W. \& Xiong, J. TaxonKit: a cross-platform and efficient NCBI taxonomy toolkit. bioRxiv 513523 (2019).

135. Papagianni, M. Recent advances in engineering the central carbon metabolism of industrially important bacteria. Microb. Cell Fact. 11, 50 (2012).

136. Komaki, H., Fudou, R., Iizuka, T., Nakajima, D., Okazaki, K., Shibata, D., Ojika, M. \& Harayama, S. PCR detection of type I polyketide synthase genes in myxobacteria. Appl. Environ. Microbiol. 74, 5571-5574 (2008).

137. Nolan, E. M. \& Walsh, C. T. How nature morphs peptide scaffolds into antibiotics. ChemBioChem 10, 34-53 (2009).

138. Caboche, S., Pupin, M., Leclère, V., Fontaine, A., Jacques, P. \& Kucherov, G. NORINE: A database of nonribosomal peptides. Nucleic Acids Res. 36, D326-D331 (2008).

139. Caboche, S., Leclère, V., Pupin, M., Kucherov, G. \& Jacques, P. Diversity of 
monomers in nonribosomal peptides: Towards the prediction of origin and biological activity. J. Bacteriol. 192, 5143-5150 (2010).

140. Flissi, A., Dufresne, Y., Michalik, J., Tonon, L., Janot, S., Noé, L., Jacques, P., Leclére, V. \& Pupin, M. Norine, the knowledgebase dedicated to non-ribosomal peptides, is now open to crowdsourcing. Nucleic Acids Res. 44, D1113-D1118 (2016).

141. Marchler-Bauer, A., Derbyshire, M. K., Gonzales, N. R., Lu, S., Chitsaz, F., Geer, L. Y., Geer, R. C., He, J., Gwadz, M., Hurwitz, D. I., Lanczycki, C. J., Lu, F., Marchler, G. H., Song, J. S., Thanki, N., Wang, Z., Yamashita, R. A., Zhang, D., Zheng, C. \& Bryant, S. H. CDD: NCBI's conserved domain database. Nucleic Acids Res. 43, D222-D226 (2015).

142. Barry, S. M., Kers, J. A., Johnson, E. G., Song, L., Aston, P. R., Patel, B., Krasnoff, S. B., Crane, B. R., Gibson, D. M., Loria, R. \& Challis, G. L. Cytochrome P450catalyzed L-tryptophan nitration in thaxtomin phytotoxin biosynthesis. Nat. Chem. Biol. 8, 814-816 (2012).

143. Moncrieffe, M. C., Fernandez, M. J., Spiteller, D., Matsumura, H., Gay, N. J., Luisi, B. F. \& Leadlay, P. F. Structure of the glycosyltransferase EryCIII in complex with its activating P450 homologue EryCII. J. Mol. Biol. 415, 92-101 (2012).

144. Borgman, P., Lopez, R. D. \& Lane, A. L. The expanding spectrum of diketopiperazine natural product biosynthetic pathways containing cyclodipeptide synthases. Org. Biomol. Chem. 17, 2305-2314 (2019).

145. Gu, B., He, S., Yan, X. \& Zhang, L. Tentative biosynthetic pathways of some microbial diketopiperazines. Appl. Microbiol. Biotechnol. 97, 8439-8453 (2013).

146. Skinnider, M. A., Johnston, C. W., Merwin, N. J., Dejong, C. A. \& Magarvey, N. A. Global analysis of prokaryotic tRNA-derived cyclodipeptide biosynthesis. BMC Genomics 19, 45 (2018).

147. Schultz, A. W., Oh, D. C., Carney, J. R., Williamson, R. T., Udwary, D. W., Jensen, P. R., Gould, S. J., Fenical, W. \& Moore, B. S. Biosynthesis and structures of cyclomarins and cyclomarazines, prenylated cyclic peptides of marine actinobacterial origin. J. Am. Chem. Soc. 130, 4507-4516 (2008). 
148. Loria, R., Bignell, D. R. D., Moll, S., Huguet-Tapia, J. C., Joshi, M. V., Johnson, E. G., Seipke, R. F. \& Gibson, D. M. Thaxtomin biosynthesis: The path to plant pathogenicity in the genus Streptomyces. Antonie van Leeuwenhoek, Int. J. Gen. Mol. Microbiol. 94, 3-10 (2008).

149. Schieferdecker, S., König, S., Weigel, C., Dahse, H. M., Werz, O. \& Nett, M. Structure and biosynthetic assembly of gulmirecins, macrolide antibiotics from the predatory bacterium Pyxidicoccus fallax. Chemistry 20, 15933-15940 (2014).

150. Baltz, R. H. MbtH homology codes to identify gifted microbes for genome mining. J. Ind. Microbiol. Biotechnol. 41, 357-369 (2014).

151. Farag, S., Bleich, R. M., Shank, E. A., Isayev, O., Bowers, A. A. \& Tropsha, A. Inter-Modular Linkers play a crucial role in governing the biosynthesis of nonribosomal peptides. Bioinformatics btz127 (2019).

152. McCusker, K. P. \& Klinman, J. P. Modular behavior of tauD provides insight into the origin of specificity in -ketoglutarate-dependent nonheme iron oxygenases. Proc. Natl. Acad. Sci. 106, 19791-19795 (2009).

153. Rosconi, F., Davyt, D., Martínez, V., Martínez, M., Abin-Carriquiry, J. A., Zane, H., Butler, A., de Souza, E. M. \& Fabiano, E. Identification and structural characterization of serobactins, a suite of lipopeptide siderophores produced by the grass endophyte Herbaspirillum seropedicae. Environ. Microbiol. 15, 916-927 (2013).

154. Strieker, M., Kopp, F., Mahlert, C., Essen, L. O. \& Marahiel, M. A. Mechanistic and structural basis of stereospecific $\mathrm{C} \beta$-hydroxylation in calcium-dependent antibiotic, a daptomycin-type lipopeptide. ACS Chem. Biol. 2, 187-196 (2007).

155. Hoffmann, T., Müller, S., Nadmid, S., Garcia, R. \& Müller, R. Microsclerodermins from terrestrial myxobacteria: An intriguing biosynthesis likely connected to a sponge symbiont. J. Am. Chem. Soc. 135, 16904-16911 (2013).

156. Micallef, M. L., D’Agostino, P. M., Sharma, D., Viswanathan, R. \& Moffitt, M. C. Genome mining for natural product biosynthetic gene clusters in the Subsection V cyanobacteria. BMC Genomics 16, 669 (2015).

157. Luesch, H., Hoffmann, D., Hevel, J. M., Becker, J. E., Golakoti, T. \& Moore, R. E. 
Biosynthesis of 4-methylproline in cyanobacteria: Cloning of nosE and nosF genes and biochemical characterization of the encoded dehydrogenase and reductase activities. J. Org. Chem. 68, 83-91 (2003).

158. Mareš, J., Jek, J. H., Urajová, P., Kopecký, J. \& Hrouzek, P. A hybrid non-ribosomal peptide/polyketide synthetase containing fatty-acyl ligase (Faal) synthesizes the bAmino fatty acid lipopeptides puwainaphycins in the cyanobacterium cylindrospermum alatosporum. PLoS One 9, e111904 (2014).

159. Yan, F., Auerbach, D., Chai, Y., Keller, L., Tu, Q., Hüttel, S., Glemser, A., Grab, H. A., Bach, T., Zhang, Y. \& Müller, R. Biosynthesis and Heterologous Production of Vioprolides: Rational Biosynthetic Engineering and Unprecedented 4Methylazetidinecarboxylic Acid Formation. Angew. Chemie - Int. Ed. 57, 87548759 (2018).

160. Miao, V., Coëffet-LeGal, M. F., Brian, P., Brost, R., Penn, J., Whiting, A., Martin, S., Ford, R., Parr, I., Bouchard, M., Silva, C. J., Wrigley, S. K. \& Baltz, R. H. Daptomycin biosynthesis in Streptomyces roseosporus: Cloning and analysis of the gene cluster and revision of peptide stereochemistry. Microbiology 151, 1507-1523 (2005).

161. Favrot, L., Blanchard, J. S. \& Vergnolle, O. Bacterial GCN5-Related NAcetyltransferases: From Resistance to Regulation. Biochemistry 55, 989-1002 (2016).

162. Forli, S. Epothilones: From discovery to clinical trials. Curr. Top. Med. Chem. 14, 2312-21 (2014).

163. Davis, K. E. R., Sangwan, P. \& Janssen, P. H. Acidobacteria, Rubrobacteridae and Chloroflexi are abundant among very slow-growing and mini-colony-forming soil bacteria. Environ. Microbiol. 13, 798-805 (2011).

164. Joseph, S. J., Hugenholtz, P., Sangwan, P., Osborne, C. A. \& Janssen, P. H. Laboratory cultivation of widespread and previously uncultured soil bacteria. Appl. Environ. Microbiol. 69, 7210-5 (2003).

165. Janssen, P. H., Yates, P. S., Grinton, B. E., Taylor, P. M. \& Sait, M. Improved culturability of soil bacteria and isolation in pure culture of novel members of the 
divisions Acidobacteria, Actinobacteria, Proteobacteria, and Verrucomicrobia. Appl. Environ. Microbiol. 68, 2391-6 (2002).

166. Li, Y., Li, Z., Yamanaka, K., Xu, Y., Zhang, W., Vlamakis, H., Kolter, R., Moore, B. S. \& Qian, P. Y. Directed natural product biosynthesis gene cluster capture and expression in the model bacterium Bacillus subtilis. Sci. Rep. 5, 9383 (2015).

167. Hu, Y., Nan, F., Maina, S. W., Guo, J., Wu, S. \& Xin, Z. Clone of plipastatin biosynthetic gene cluster by transformation-associated recombination technique and high efficient expression in model organism Bacillus subtilis. J. Biotechnol. 288, 18 (2018).

168. Roulet, J., Taton, A., Golden, J. W., Arabolaza, A., Burkart, M. D. \& Gramajo, H. Development of a cyanobacterial heterologous polyketide production platform. Metab. Eng. 49, 94-104 (2018).

169. Fierer, N., Bradford, M. A. \& Jackson, R. B. Toward an ecological classification of soil bacteria. Ecology 88, 1354-64 (2007).

170. Sabín, J. G. Exploring Novel Opportunities for Aureolic Acids as Anticancer Drugs. Biochem. Pharmacol. Open Access 02, 1-3 (2013).

171. Méndez, C., González-Sabín, J., Morís, F. \& Salas, J. A. Expanding the Chemical Diversity of the Antitumoral Compound Mithramycin by Combinatorial Biosynthesis and Biocatalysis: The Quest for Mithralogs with Improved Therapeutic Window. Planta Med. 81, 1326-1338 (2015).

172. Ralifo, P. \& Crews, P. A New Structural Theme in the Imidazole-Containing Alkaloids from a Calcareous Leucetta Sponge. J. Org. Chem. 69, 9025-9029 (2004).

173. Molinski, T. F. \& Morinaka, B. I. Integrated approaches to the configurational assignment of marine natural products. Tetrahedron 68, 9307-9343 (2012).

174. Omura, S., Tanaka, H., Iwai, Y., Nishigaki, K., Awaya, J., Takahashi, Y. \& Masuma, R. A new antibiotic, setomimycin, produced by a strain of Streptomyces. J. Antibiot. (Tokyo). 31, 1091-1098 (1978).

175. Kakinuma, K., Imamura, N., Ikekawa, N., Tanaka, H., Minami, S. \& Omura, S. Structure and Biosynthesis of Setomimycin. A Novel 9,9'-Bianthryl Antibiotic. $J$. Am. Chem. Soc. 102, 7493-7498 (1980). 
176. Präg, A., Grüning, B. A., Häckh, M., Lüdeke, S., Wilde, M., Luzhetskyy, A., Richter, M., Luzhetska, M., Günther, S. \& Müller, M. Regio- and stereoselective intermolecular oxidative phenol coupling in streptomyces. J. Am. Chem. Soc. 136, 6195-6198 (2014).

177. Piel, J., Hertweck, C., Shipley, P. R., Hunt, D. M., Newman, M. S. \& Moore, B. S. Cloning, sequencing and analysis of the enterocin biosynthesis gene cluster from the marine isolate 'Streptomyces maritimus': Evidence for the derailment of an aromatic polyketide synthase. Chem. Biol. 7, 943-955 (2000).

178. Villebro, R., Shaw, S., Blin, K. \& Weber, T. Sequence-based classification of type II polyketide synthase biosynthetic gene clusters for antiSMASH. J. Ind. Microbiol. Biotechnol. 46, 469-475 (2019).

179. Caldara-Festin, G., Jackson, D. R., Barajas, J. F., Valentic, T. R., Patel, A. B., Aguilar, S., Nguyen, M. C., Vo, M., Khanna, A., Sasaki, E., Liu, H. W., Tsai, S. C. \& Smith, J. L. Structural and functional analysis of two di-domain aromatase/cyclases from type II polyketide synthases. Proc. Natl. Acad. Sci. U. S. A. 112, E6844-E6851 (2015).

180. Shaaban, K. A., Shaaban, M., Grün-Wollny, I., Maier, A., Fiebig, H. H. \& Laatsch, H. Julichrome Q6 glucuronide, a monomeric subunit of the julimycin B-I complex from a terrestrial Streptomyces sp. J. Nat. Prod. 70, 1545-1550 (2007).

181. Staley, A. L. \& Rinehart, K. L. Spectomycins, new antibacterial compounds produced by Streptomyces spectabilis: isolation, structures, and biosynthesis. $J$. Antibiot. (Tokyo). 47, 1425-1433 (2012).

182. Lipinski, C. A., Lombardo, F., Dominy, B. W. \& Feeney, P. J. Experimental and computational approaches to estimate solubility and permeability in drug discovery and development settings. Adv. Drug Deliv. Rev. 64, 4-17 (2012).

183. Lombó, F., Braña, A. F., Méndez, C. \& Salas, J. A. The mithramycin gene cluster of Streptomyces argillaceus contains a positive regulatory gene and two repeated DNA sequences that are located at both ends of the cluster. J. Bacteriol. 181, 642-647 (1999).

184. Brazhnikova, M. G., Krugliak, E. B., Kovsharova, I. N., Konstantinova, N. V \& 
Proshilakova, V. V. Isolation, purification and study on some physico-chemical properties of a new antibiotic olivomycin. Antibiotiki 7, 39-44 (1962).

185. Jayasuriya, H., Lingham, R. B., Graham, P., Quamina, D., Herranz, L., Genilloud, O., Gagliardi, M., Danzeisen, R., Tomassini, J. E., Zink, D. L., Guan, Z. \& Singh, S. B. Durhamycin A, a potent inhibitor of HIV Tat transactivation. J. Nat. Prod. 65, 1091-1095 (2002).

186. Ogawa, H., Yamashita, Y., Katahira, R., Chiba, S., Iwasaki, T., Ashizawa, T. \& Nakano, H. UCH9, a New Antitumor Antibiotic Produced by Streptomyces: I. Producing Organism, Fermentation Isolation and Biological Activities. J. Antibiot. (Tokyo). 51, 261-266 (1998).

187. Berlin, Y. A., Kolosov, M. N., Vasina, I. V. \& Yartseva, I. V. The structure of chromocyclomycin. Chem. Commun. 762-763 (1968).

188. Bhave, S. V., Sawant, A. V., Shanbhag, P., Parab, R. R., Ranadive, P. V., Mishra, P. D. \& Mahajan, G. B. Fermentation, Isolation of Mithramycin from Streptomyces of Playa Region and its Novel Anti- MRSA and Anti-VRE Activity. SOJ Microbiol. Infect. Dis. 3, 01-08 (2015).

189. Remsing, L. L., González, A. M., Nur-e-Alam, M., Fernández-Lozano, M. J., Braña, A. F., Rix, U., Oliveira, M. A., Méndez, C., Salas, J. A. \& Rohr, J. Mithramycin SK, a novel antitumor drug with improved therapeutic index, mithramycin SA, and demycarosyl-mithramycin SK: Three new products generated in the mithramycin producer streptomyces argillaceus through combinatorial biosynthesis. J. Am. Chem. Soc. 125, 5745-5753 (2003).

190. Hou, M. H. \& Wang, A. H.-J. Mithramycin forms a stable dimeric complex by chelating with $\mathrm{Fe}(\mathrm{II})$ : DNA-interacting characteristics, cellular permeation and cytotoxicity. Nucleic Acids Res. 33, 1352-1361 (2005).

191. Tevyashova, A. N., Shtil, A. A., Olsufyeva, E. N., Luzikov, Y. N., Reznikova, M. I., Dezhenkova, L. G., Isakova, E. B., Bukhman, V. M., Durandin, N. A., Vinogradov, A. M., Kuzmin, V. A. \& Preobrazhenskaya, M. N. Modification of olivomycin A at the side chain of the aglycon yields the derivative with perspective antitumor characteristics. Bioorganic Med. Chem. 19, 7387-7393 (2011). 
192. Hsu, C. W., Chuang, S. M., Wu, W. L. \& Hou, M. H. The Crucial Role of Divalent Metal Ions in the DNA-Acting Efficacy and Inhibition of the Transcription of Dimeric Chromomycin A3. PLoS One 7, e43792 (2012).

193. Scott, D., Chen, J. M., Bae, Y. \& Rohr, J. Semi-synthetic mithramycin SA derivatives with improved anticancer activity. Chem. Biol. Drug Des. 81, 615-624 (2013).

194. Abdelfattah, M. S. \& Rohr, J. Premithramycinone G, an early shunt product of the mithramycin biosynthetic pathway accumulated upon inactivation of oxygenase MtmOII. Angew. Chemie - Int. Ed. 45, 5685-5689 (2006).

195. Kunnari, T., Klika, K. D., Blanco, G., Méndez, C., Mäntsälä, P., Hakala, J., Sillanpää, R., Tähtinen, P., Salas, J. \& Ylihonko, K. Hybrid compounds generated by the introduction of a nogalamycin-producing plasmid into Streptomyces argillaceus. J. Chem. Soc., Perkin Trans. 1 1818-1825 (2002).

196. Baig, I., Perez, M., Braña, A. F., Gomathinayagam, R., Damodaran, C., Salas, J. A., Méndez, C. \& Rohr, J. Mithramycin Analogues Generated by Combinatorial Biosynthesis Show Improved Bioactivity. J. Nat. Prod. 71, 199-207 (2008).

197. Núñez, L. E., Nybo, S. E., González-Sabín, J., Pérez, M., Menéndez, N., Braña, A. F., Shaaban, K. A., He, M., Morís, F., Salas, J. A., Rohr, J. \& Méndez, C. A Novel Mithramycin Analogue with High Antitumor Activity and Less Toxicity Generated by Combinatorial Biosynthesis. J. Med. Chem. 55, 5813-5825 (2012).

198. Menéndez, N., Braña, A. F., Salas, J. A. \& Méndez, C. Involvement of a chromomycin $\mathrm{ABC}$ transporter system in secretion of a deacetylated precursor during chromomycin biosynthesis. Microbiology 153, 3061-3070 (2007).

199. Fernández, E., Lombó, F., Méndez, C. \& Salas, J. A. An ABC transporter is essential for resistance to the antitumor agent mithramycin in the producer Streptomyces argillaceus. Mol. Gen. Genet. 251, 692-698 (1996).

200. Wohlert, S. E., Künzel, E., Machinek, R., Méndez, C., Salas, J. A. \& Rohr, J. The structure of mithramycin reinvestigated. J. Nat. Prod. 62, 119-121 (1999).

201. Herai, S., Hashimoto, Y., Higashibata, H., Maseda, H., Ikeda, H., Omura, S. \& Kobayashi, M. Hyper-inducible expression system for streptomycetes. Proc. Natl. 
Acad. Sci. 101, 14031-14035 (2004).

202. Stajner, K., Blumauerová, M., Callieri, D. A. S. \& Vaněk, Z. The study of variability and strain selection in Streptomyces atroolivaceus - II. Chromatographic analysis of mithramycin-producing and non-producing strains. Folia Microbiol. (Praha). 19, 498-506 (1974).

203. Berlin, Y. A., Kolosov, M. N. \& Severtsova, I. V. Olivomycin and related antibiotics XXX. The structure of chromocyclin. Chem. Nat. Compd. 9, 492-497 (1975).

204. Berlin, Y. A., Kolosov, M. N. \& Yartseva, I. V. Olivomycin and related antibiotics XXXII. The structure of chromocyclomycin. Chem. Nat. Compd. 9, 504-508 (1973).

205. Gordon, M. A. \& Lapa, E. W. Durhamycin, a Pentaene Antifungal Antibiotic from Streptomyces durhamensis sp. n. Appl. Microbiol. 14, 754-75460 (1966).

206. Prado, L., Fernández, E., Weißbach, U., Blanco, G., Quirós, L. M., Braña, A. F., Méndez, C., Rohr, J. \& Salas, J. A. Oxidative cleavage of premithramycin B is one of the last steps in the biosynthesis of the antitumor drug mithramycin. Chem. Biol. 6, 19-30 (1999).

207. Fernández, E., Weissbach, U., Sánchez Reillo, C., Braña, A. F., Méndez, C., Rohr, J. \& Salas, J. A. Identification of two genes from Streptomyces argillaceus encoding glycosyltransferases involved in transfer of a disaccharide during biosynthesis of the antitumor drug mithramycin. J. Bacteriol. 180, 4929-37 (1998).

208. Braine, T. Race against time to develop new antibiotics. Bull. World Health Organ. 89, 88-89 (2011).

209. Fierer, N. Embracing the unknown: Disentangling the complexities of the soil microbiome. Nat. Rev. Microbiol. 15, 579-590 (2017).

210. Parsley, L. C., Linneman, J., Goode, A. M., Becklund, K., George, I., Goodman, R. M., Lopanik, N. B. \& Liles, M. R. Polyketide synthase pathways identified from a metagenomic library are derived from soil Acidobacteria. FEMS Microbiol. Ecol. 78, 176-187 (2011).

211. Cummings, M., Peters, A. D., Whitehead, G. F. S., Menon, B. R. K., Micklefield, J., Webb, S. J. \& Takano, E. Assembling a plug-and-play production line for combinatorial biosynthesis of aromatic polyketides in Escherichia coli. PLOS Biol. 
17, e3000347 (2019).

212. Garcia-Bernardo, J., Braña, A. F., Méndez, C. \& Salas, J. A. Insertional inactivation of mtrX and mtrY genes from the mithramycin gene cluster affects production and growth of the producer organism Streptomyces argillaceus. FEMS Microbiol. Lett. 186, 61-65 (2000). 


\section{Appendix A. Computational scripts}

\section{decontam.sh}

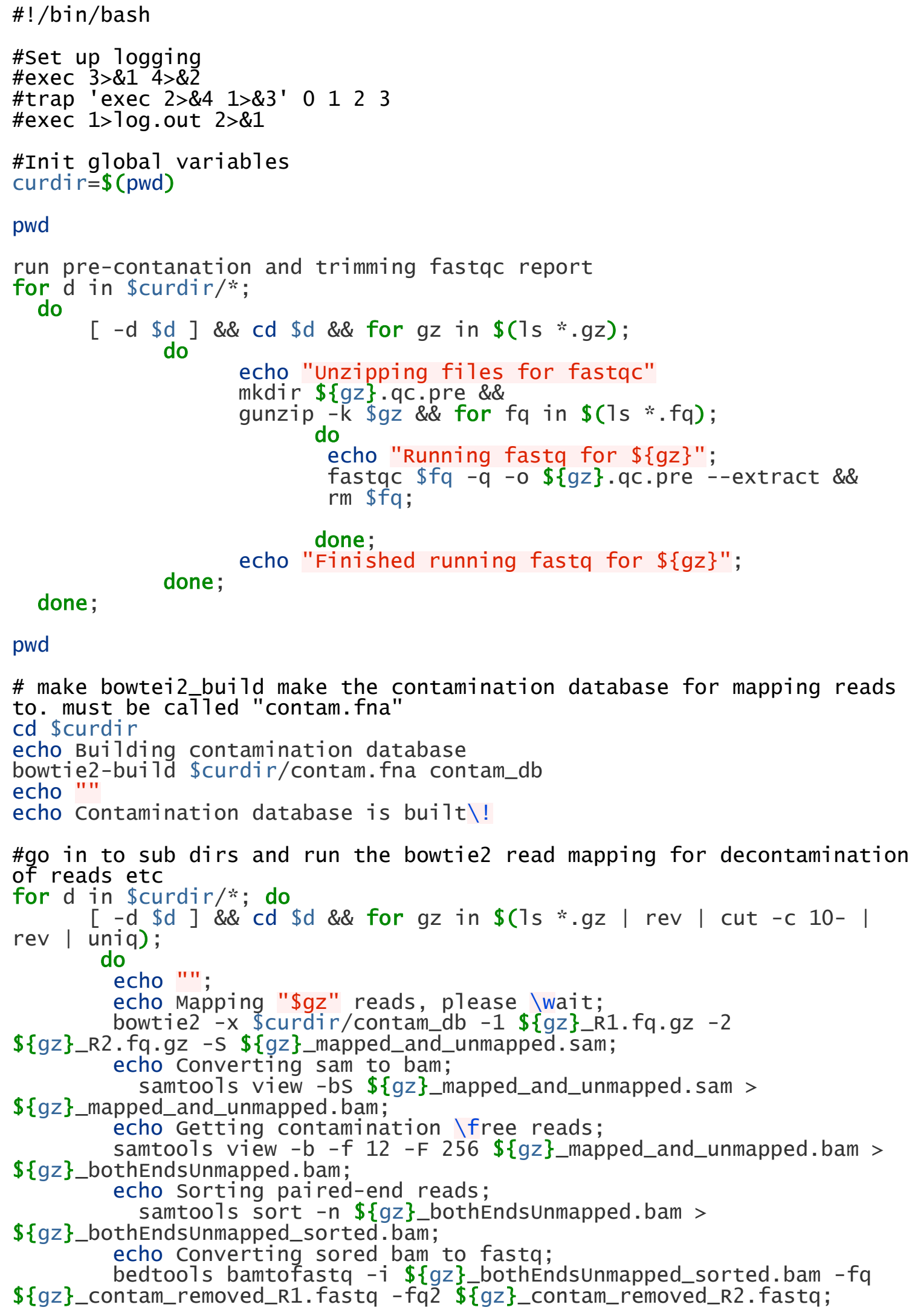


done;

echo cleaning up alignments to save disk space;

$\mathrm{rm} *$ bam \&\& rm *.sam;

done;

pwd

\#in curdir, clean up bowtie2 index

cd \$curdir

pwd

echo "cleaning up contamination database."

rm \$curdir/*.bt2

\#\#\#Run trimming on decontaninated reads with trimmomatic for $d$ in \$curdir/*;

do

[ - d $\$ d$ ] \&\& cd $\$ d \& \&$ for fq in $\$(1 s *$ fastq $\mid$ rev | cut $-c 10-1$

rev ( uniq); do

echo "Running Trimmomatic"

sleep 2

trimmomatic PE -threads $4 \$\{f q\}$ R1.fastq $\$\{f q\}$ R2.fastq -baseout

$\$\{\mathrm{fq}\}$. trimmed.fq ILLUMINACLIP:/home/miniconda3/pkgs/trimmomatic-0.38-

0/share/trimmomatic-0.38-0/adapters/TruSeq3-PE.fa:2:30:10:4:4: \true

LEADING: 9 TRAILING: 9 SLIDINGWINDOW: $4: 15$ MINLEN: 36 ;

rm $\$\{f q\} \_R 1$. fastq $\$\{f q\} \_R 2$. fastq

$\mathrm{rm} * \mathrm{U} . \mathrm{fq}$

done;

done;

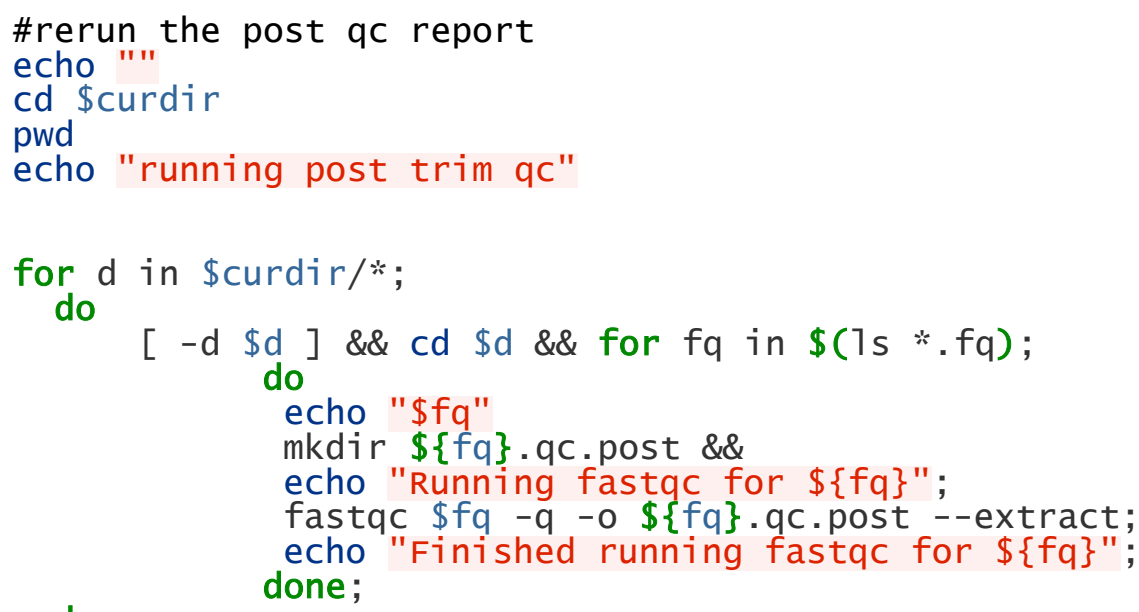

\#Clean up the qc reports and read files

cd \$curdir

echo "

echo "cleaning up and moving files around"

echo "

for d in \$curdir/*;

do

$[-d \$ d] \& \& c d \$ d \& \&$

mkdir qc_reports assemb7y \&\& mv *.pre qc_reports \&\& mv*.post qc_reports $\& \& \mathrm{mv} *$. fq assemb7y \&\& cd assemb7y \&\&

for files in $\$(1 \mathrm{~s} * \mathrm{fq}|\mathrm{rev}|$ cut $-\mathrm{c} 7-\mid$ rev $\mid$ uniq ); do

done;

fq2fa--merge $\$\{f i\rceil e s\} \_1 P . f q \$\{f i 1 e s\} \_2 P . f q ~ \$ f i 1 e s . R 1-R 2 . f a ;$

done; 


\section{gbk_to_faa.py}

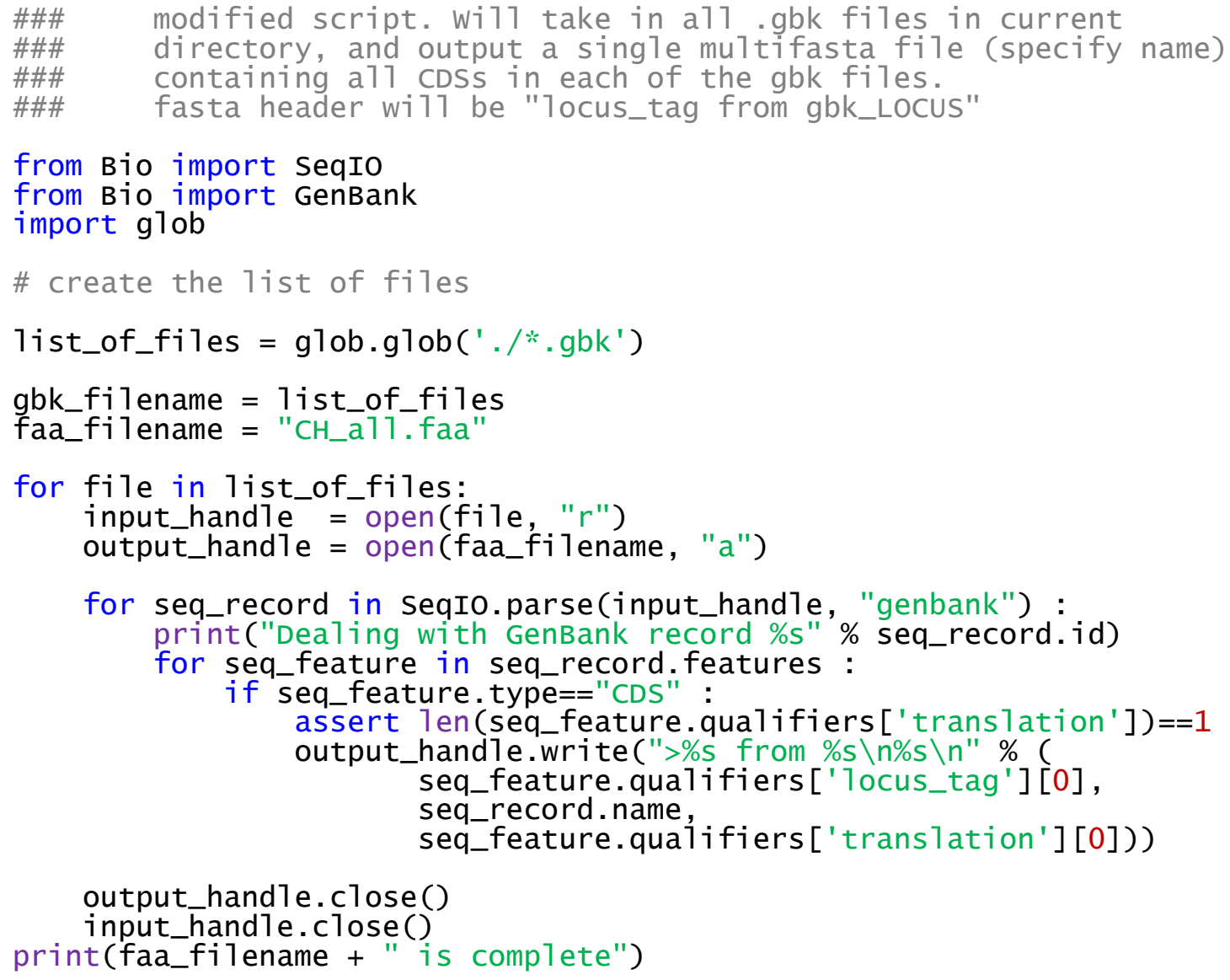

\section{allsoil_taxa.ipynb}

import pandas as pd
import numpy as np
import altair as alt

a1t.data_transformers.enable('defau1t', max_rows=None)

DataTransformerRegistry.enable('defau1t')

\#Loading the diamond blast taxonomy.tsv files

$\mathrm{CH}=$ pd.read_csv('CH_taxa.tsv', sep $=$ '\t')

$\mathrm{HM}=$ pd.read_csv ('HM_taxa.tsv', sep $\left.={ }^{\prime} \backslash \mathrm{t}^{\prime}\right)$

$\mathrm{RX}=$ pd.read_csv('RX_taxa.tsv', sep $\left.={ }^{\prime} \backslash \mathrm{t}^{\prime}\right)$

$\mathrm{SL}=$ pd.read_csv('SL_taxa.tsv', sep $=$ '\t')

$\mathrm{RU}=$ pd.read_csv('RU_taxa.tsv', sep='\t')

MIBiG = pd.read_csv('MIBiG_taxa.tsv', sep='\t')

NCBI $=$ pd.read_csv('NCBI_taxa.tsv', sep=' $\left.\backslash t^{\prime}\right)$

\#Loading in 7ists of taxIDs associated with each phy7um (and other note worthy divisions) from.txt files generated by Taxonkit \#Defining 7abe7_phy7a() function to classify a taxID to a particular ph yla/division, or $x$ if unknown

Rokubacteria $=[2053607]$

PVC $=[1783257,641407]$

$\mathrm{FCB}=[1783270]$

Terrabacteria $=[1783272,2219043,187137]$

Generic $=[0,1,2,77133,131567,1869227,2035431,1855372,1037453$, 
$1091571,1652106,312019,1091567,155900,176282,48479,684592]$ with open ('acidobacteria.txt') as $f$ : Acidobacteria $=[($ line.strip()) for line in $f$ if 1ine.strip() $]$ Acidobacteria $=$ [int( $i$ ) for $i$ in Acidobacteria]

with open('actinobacteria.txt') as $f$ : Actinobacteria $=[(1$ ine.strip()) for line in $f$ if 1ine.strip()] Actinobacteria $=$ [int( $i$ ) for $i$ in Actinobacteria]

with open ('bacteroidetes.txt') as $f$ : Bacteroidetes $=[(1$ ine.strip()) for line in $f$ if 1ine.strip()] Bacteroidetes $=$ [int $(i)$ for $i$ in Bacteroidetes]

with open ('chloroflexi.txt') as $f$ : Chloroflexi $=[(1$ ine.strip()) for line in $f$ if line.strip() $]$ chloroflexi $=$ [int( $i$ ) for $i$ in chloroflexi]

with open ('cyanobacteria.txt') as $f$ : Cyanobacteria $=[(1$ ine.strip()) for 1ine in f if 1ine.strip() $]$ cyanobacteria $=$ [int( $i)$ for $i$ in Cyanobacteria]

with open('firmicutes.txt') as $f$ : Firmicutes $=[(1$ ine.strip()) for line in $f$ if 1ine.strip()] Firmicutes $=$ [int $(i)$ for $i$ in Firmicutes $]$

with open ('gemmatimonadetes.txt') as $f$ : Gemmatimonadetes $=[(1$ ine.strip()) for line in $f$ if 1ine.strip()] Gemmatimonadetes $=$ [int( $i$ ) for $i$ in Gemmatimonadetes]

with open('nitrospirae.txt') as $f$ : Nitrospirae $=[($ line.strip()) for line in $f$ if line.strip() $]$ Nitrospirae $=$ [int( $i$ ) for $i$ in Nitrospirae]

with open('planctomycetes. txt') as $f$ : Planctomycetes $=[(1$ ine.strip()) for line in $f$ if line.strip() $]$ $\mathrm{Pl}$ anctomycetes $=[$ int $(i)$ for $i$ in Planctomycetes $]$

with open('proteobacteria.txt') as $f$ : Proteobacteria $=[(1$ ine.strip()) for line in $f$ if line.strip()] Proteobacteria $=$ [int $(i)$ for $i$ in Proteobacteria]

with open ('verrucomicrobja.txt') as $f$ : verrucomicrobia $=[(1$ ine.strip()) for line in $f$ if line.strip()) verrucomicrobia $=[$ int $(i)$ for $i$ in verrucomicrobia $]$

with open ('fungi.txt') as $f$ : Fungi $=[(1 i n e \cdot s t r i p())$ for line in $f$ if 1ine.strip() $]$ Fungi $=$ [int( $i$ ) for $i$ in Fungi $]$

def 1abe1_phyla (row):

if row ['taxID'] in Acidobacteria: return 'Acidobacteria'

if row ['taxID'] in verrucomicrobia: return 'Verrucomicrobia'

if row['taxID'] in Gemmatimonadetes: return 'Gemmatimonadetes'

if row['taxid'] in Rokubacteria: return 'Rokubacteria'

if row['taxID'] in Actinobacteria: return 'Actinobacteria'

if row ['taxID'] in Proteobacteria: return 'Proteobacteria'

if row ['taxID'] in Planctomycetes: return 'Planctomycetes'

if row['taxID'] in chloroflexi: return 'chloroflexi'

if row ['taxID'] in Cyanobacteria: return 'Cyanobacteria'

if row ['taxID'] in Bacteroidetes: return 'Bacteroidetes'

if row ['taxID'] in Nitrospirae: return 'Nitrospirae

if row ['taxID'] in PVC: return 'PVC'

if row ['taxID'] in Fungi: return 'Fungi'

if row['taxid'] in FCB: return 'FCB'

if row['taxID'] in Firmicutes: 


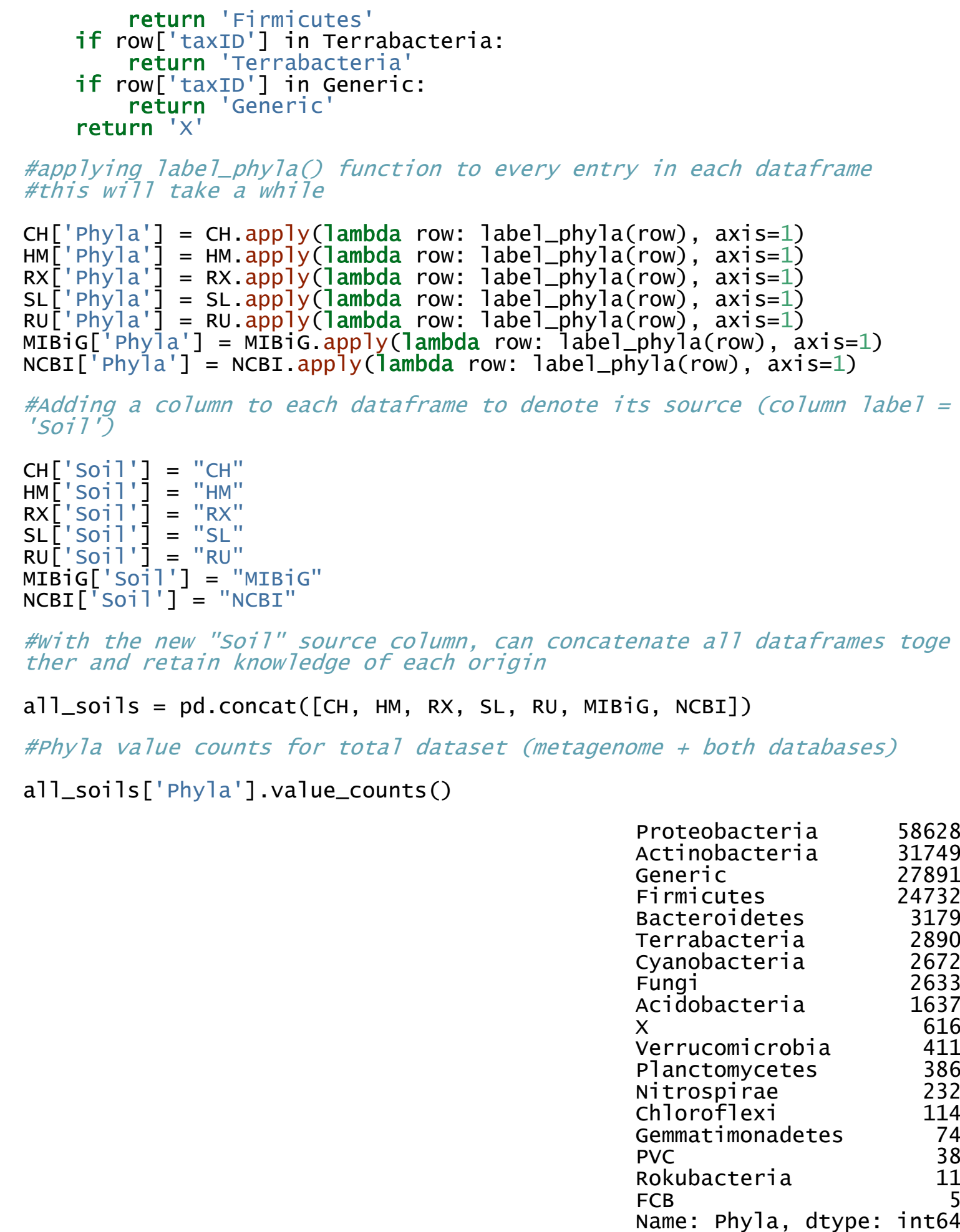

\#deleting the above phyla 7eve7 classifications (X, Generic, PVC, Terra bacteria) and 7ow abundance (Rokubacteria)

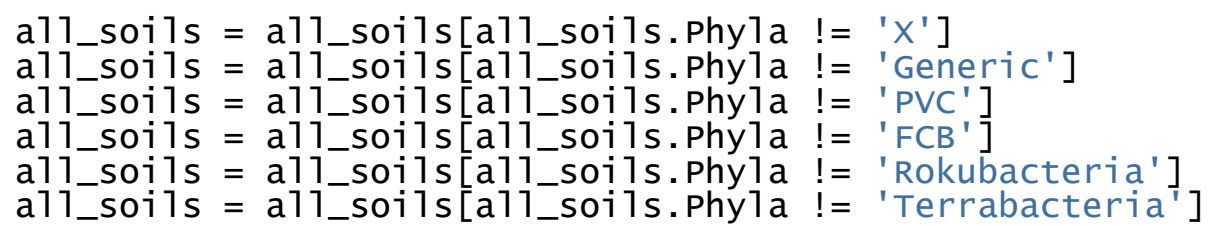


\#Phy7a value counts for total dataset after pruning

a11_soi1s['Phyla'].value_counts()

$\begin{array}{lr}\text { Proteobacteria } & 58628 \\ \text { Actinobacteria } & 31749 \\ \text { Firmicutes } & 24732 \\ \text { Bacteroidetes } & 3179 \\ \text { Cyanobacteria } & 2672 \\ \text { Fungi } & 2633 \\ \text { Acidobacteria } & 1637 \\ \text { Verrucomicrobia } & 411 \\ \text { Planctomycetes } & 386 \\ \text { Nitrospirae } & 232 \\ \text { Chloroflexi } & 114 \\ \text { Gemmatimonadetes } & 74 \\ \text { Name: Phyla, dtype: int64 }\end{array}$

\section{\#P7otting}

a1t.Chart(a11_soi1s).mark_bar().encode(

$x=a 7 t . x($ 'count (Phyla) ', stack="norma7ize"),

]),

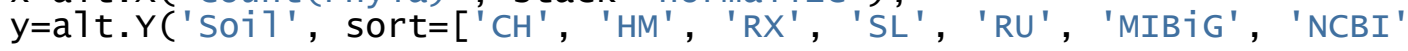

color=alt. Color ('Phyla', scale=alt. Scale $($ scheme $=$ ' tableau20' $)$ ),

order=alt.order('Phyla', sort='ascending')

). properties (height $=150$, width=300)

png

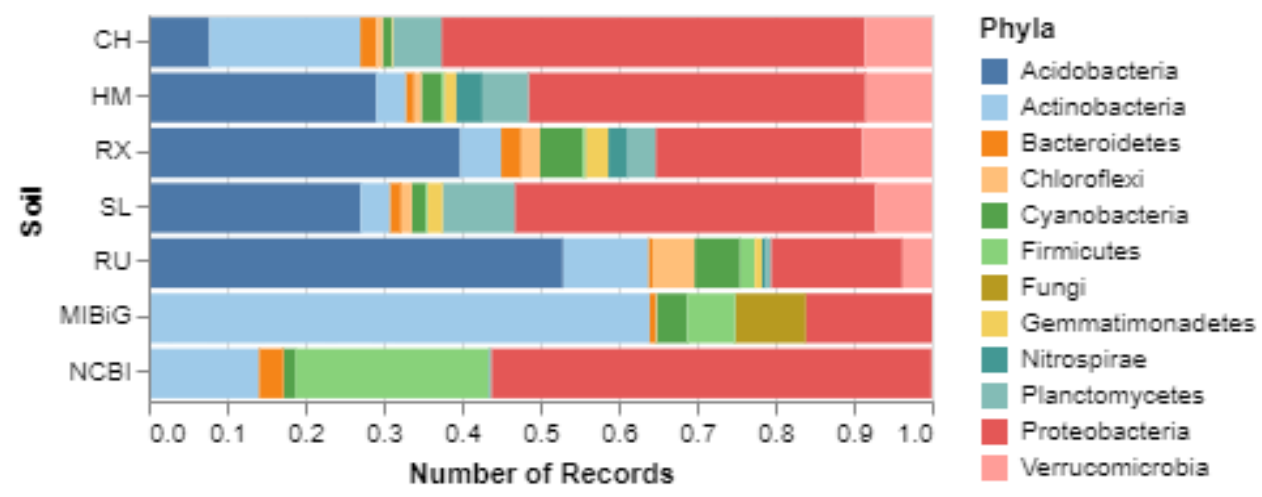


Appendix B. NMR spectra for compound 1

${ }^{1} \mathrm{H}$ spectrum of compound $1\left(600 \mathrm{MHz}, \mathrm{CDCl}_{3}\right)$

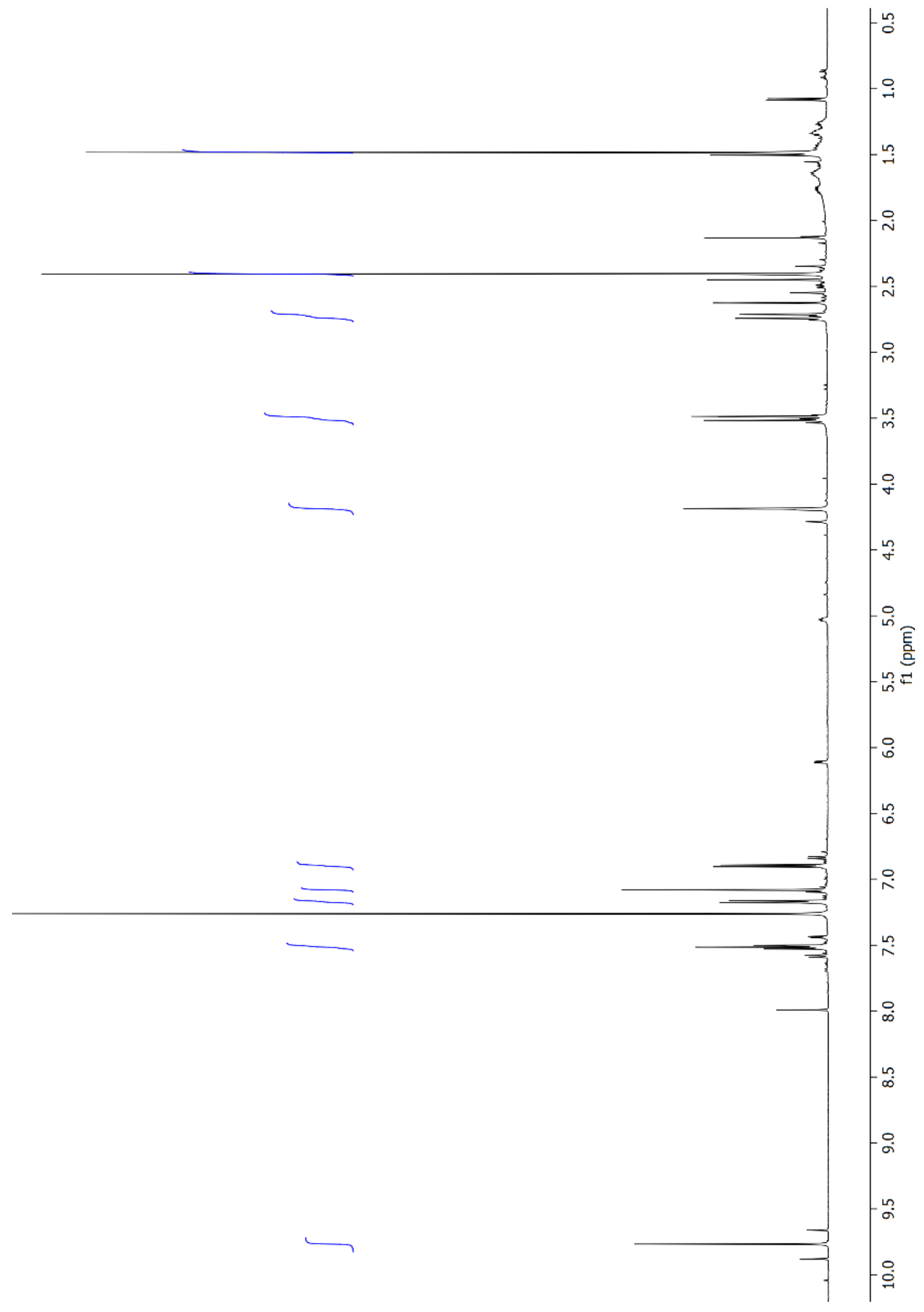




\section{${ }^{13} \mathrm{C}$ spectrum of compound $1\left(150 \mathrm{MHz}, \mathrm{CDCl}_{3}\right)$}

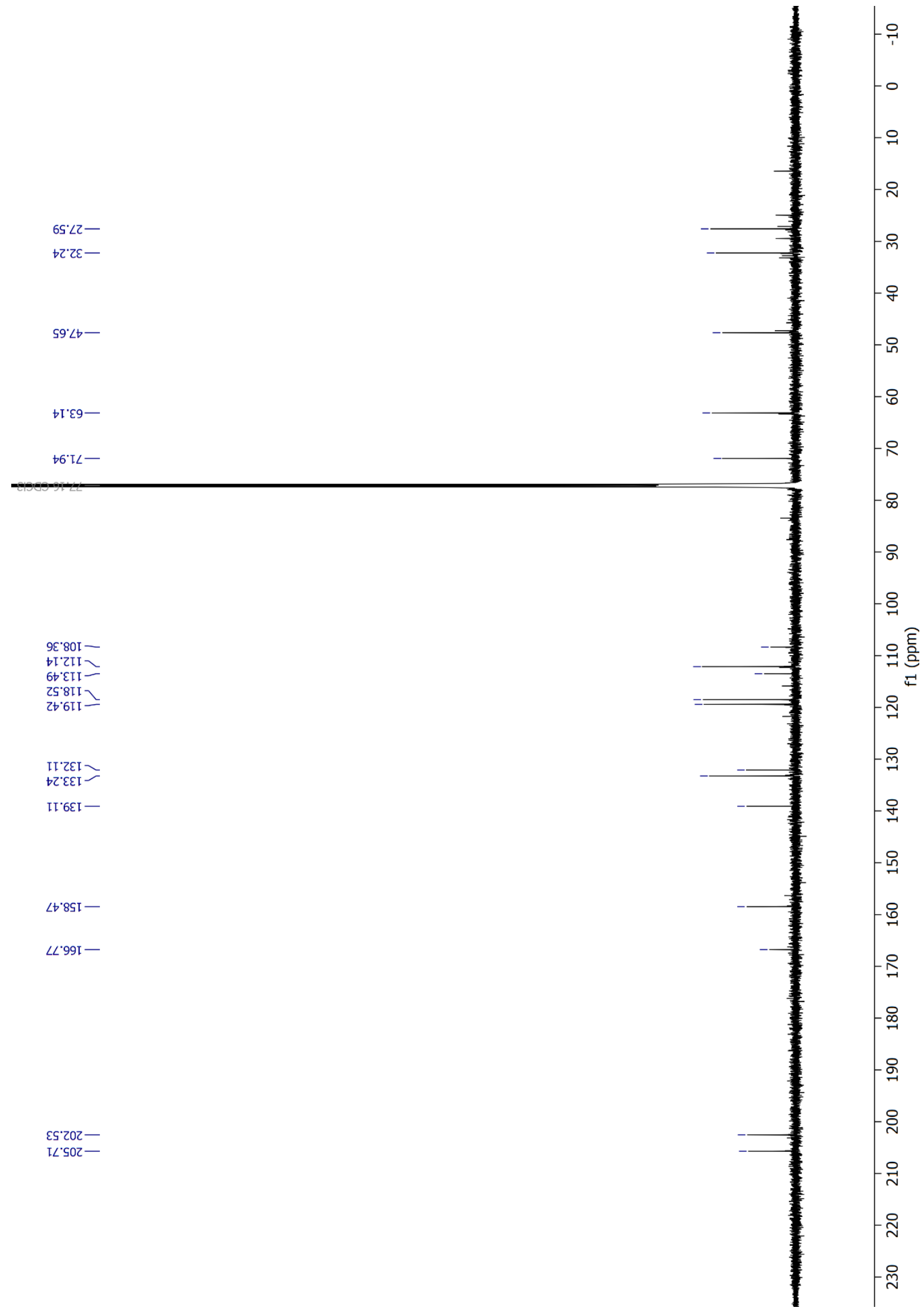


COSY spectrum of compound $1\left(600 \mathrm{MHz}, \mathrm{CDCl}_{3}\right)$

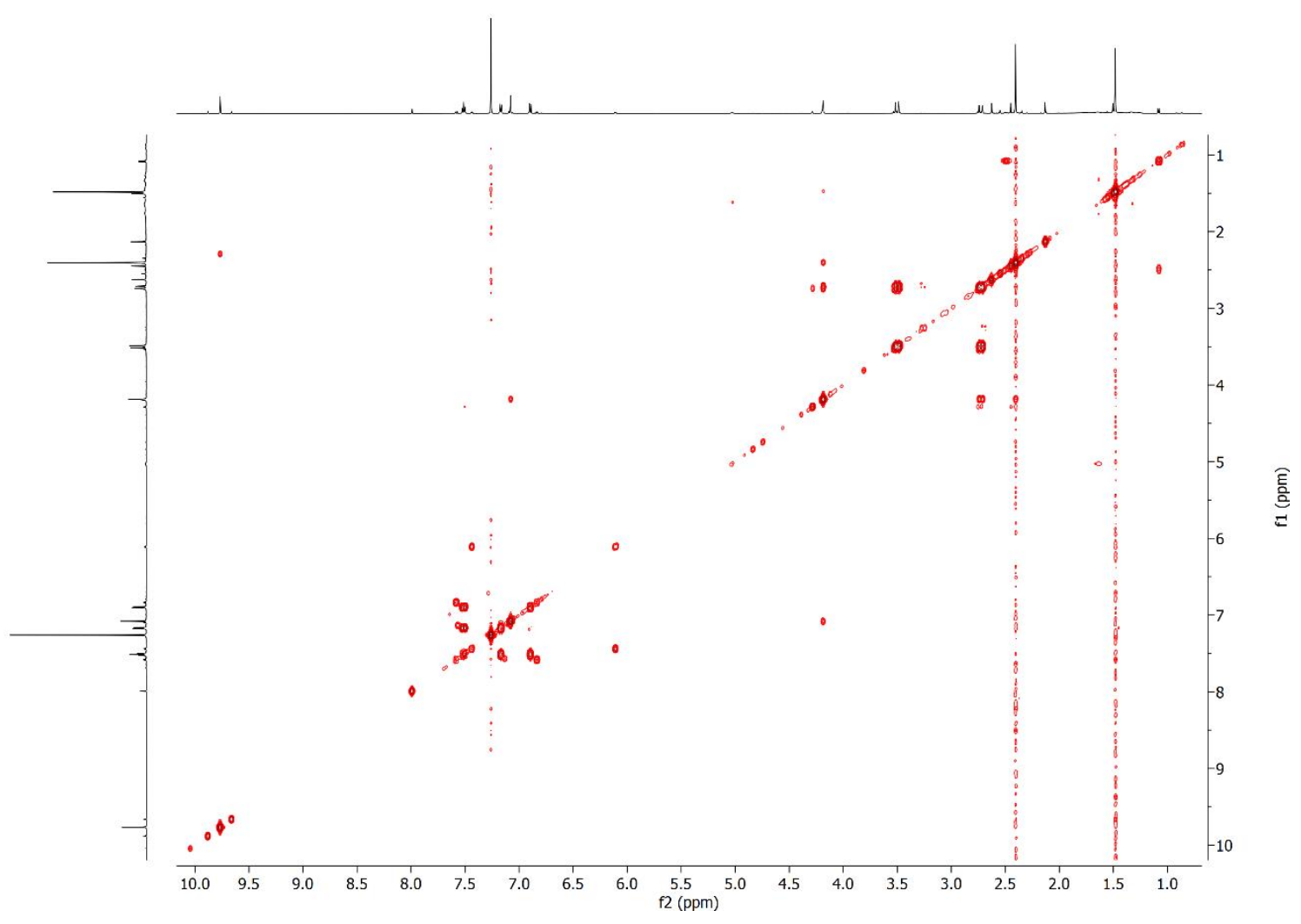

HSQC spectrum of compound $1\left(600 \mathrm{MHz}, \mathrm{CDCl}_{3}\right)$

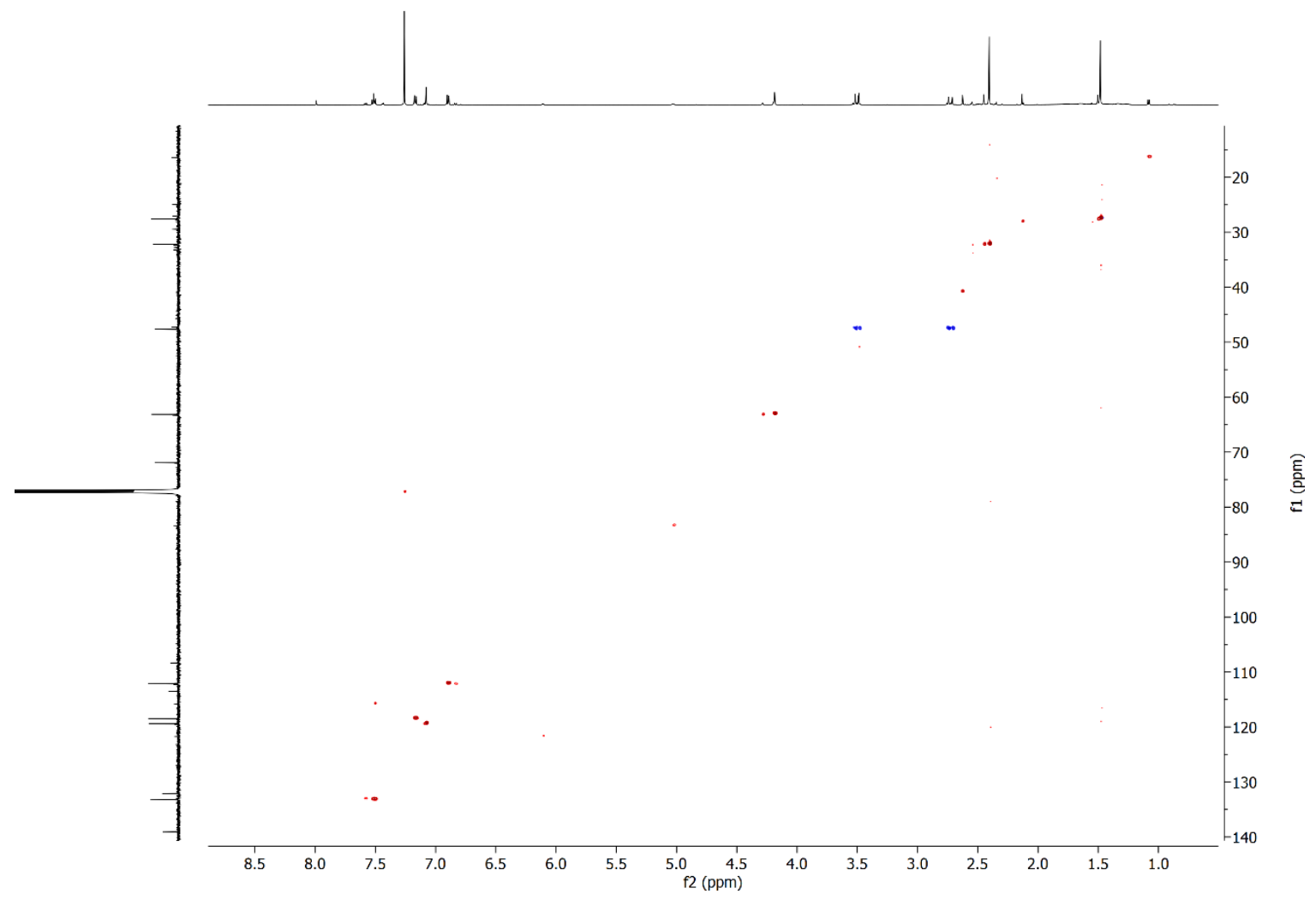


HMBC spectrum of compound $1\left(600 \mathrm{MHz}, \mathrm{CDCl}_{3}\right)$

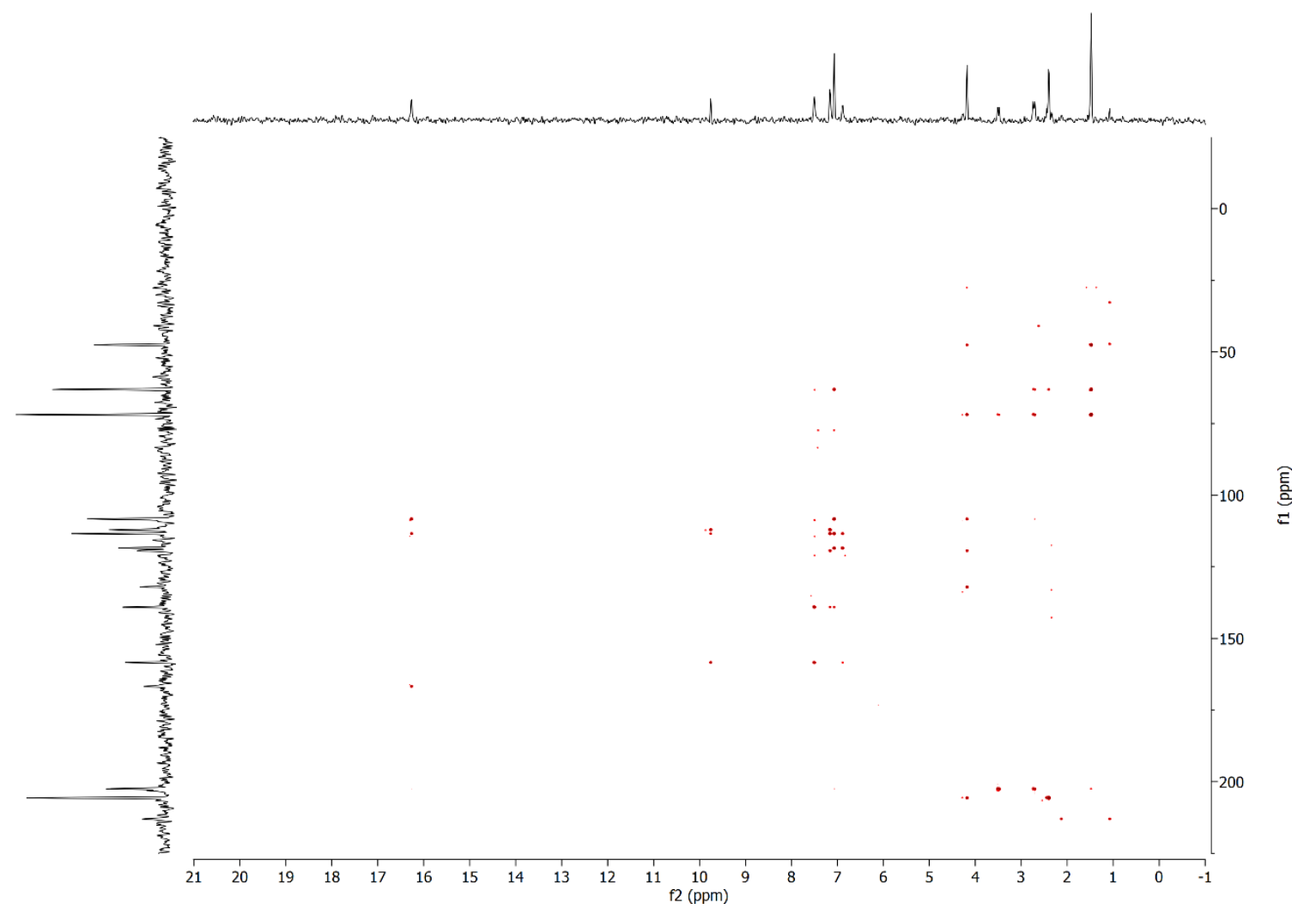




\section{Appendix C. NMR spectra of compound 2}

(note incomplete spectra/degraded compound)

${ }^{1} \mathrm{H}$ spectrum of compound $2\left(600 \mathrm{MHz}, \mathrm{CDCl}_{3}\right)$

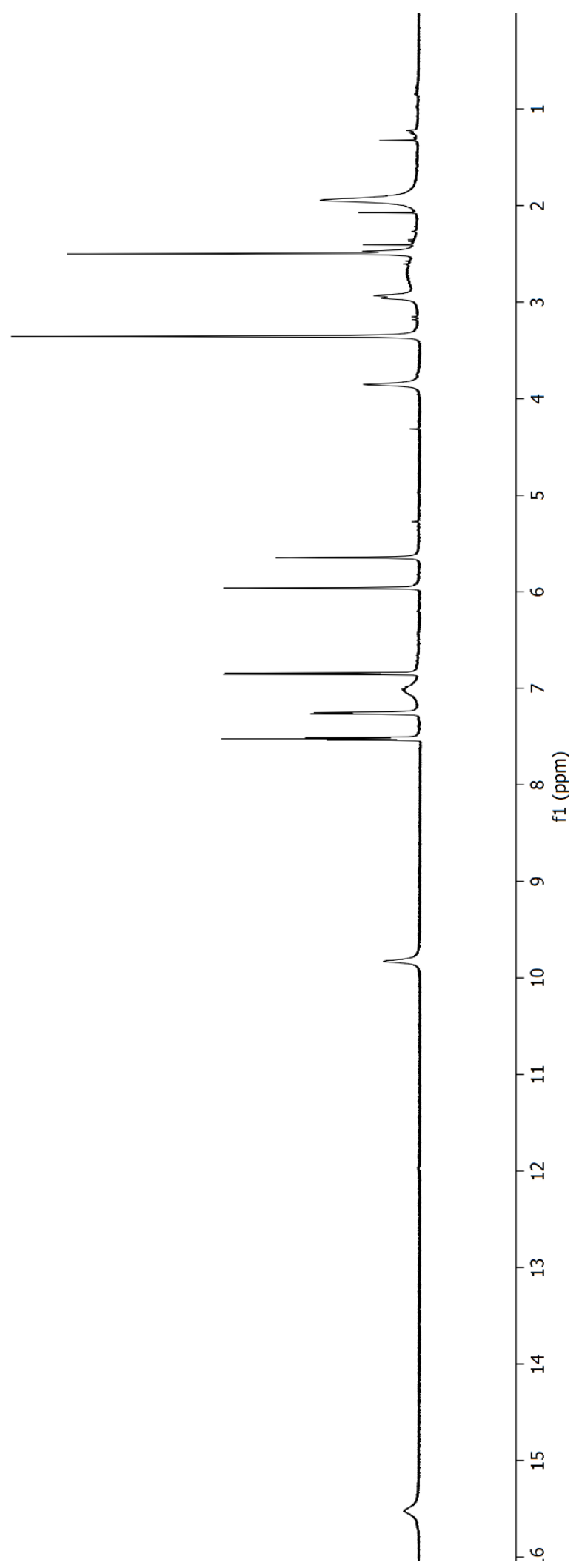


${ }^{13} \mathrm{C}$ spectrum of compound $2\left(150 \mathrm{MHz}, \mathrm{CDCl}_{3}\right)$

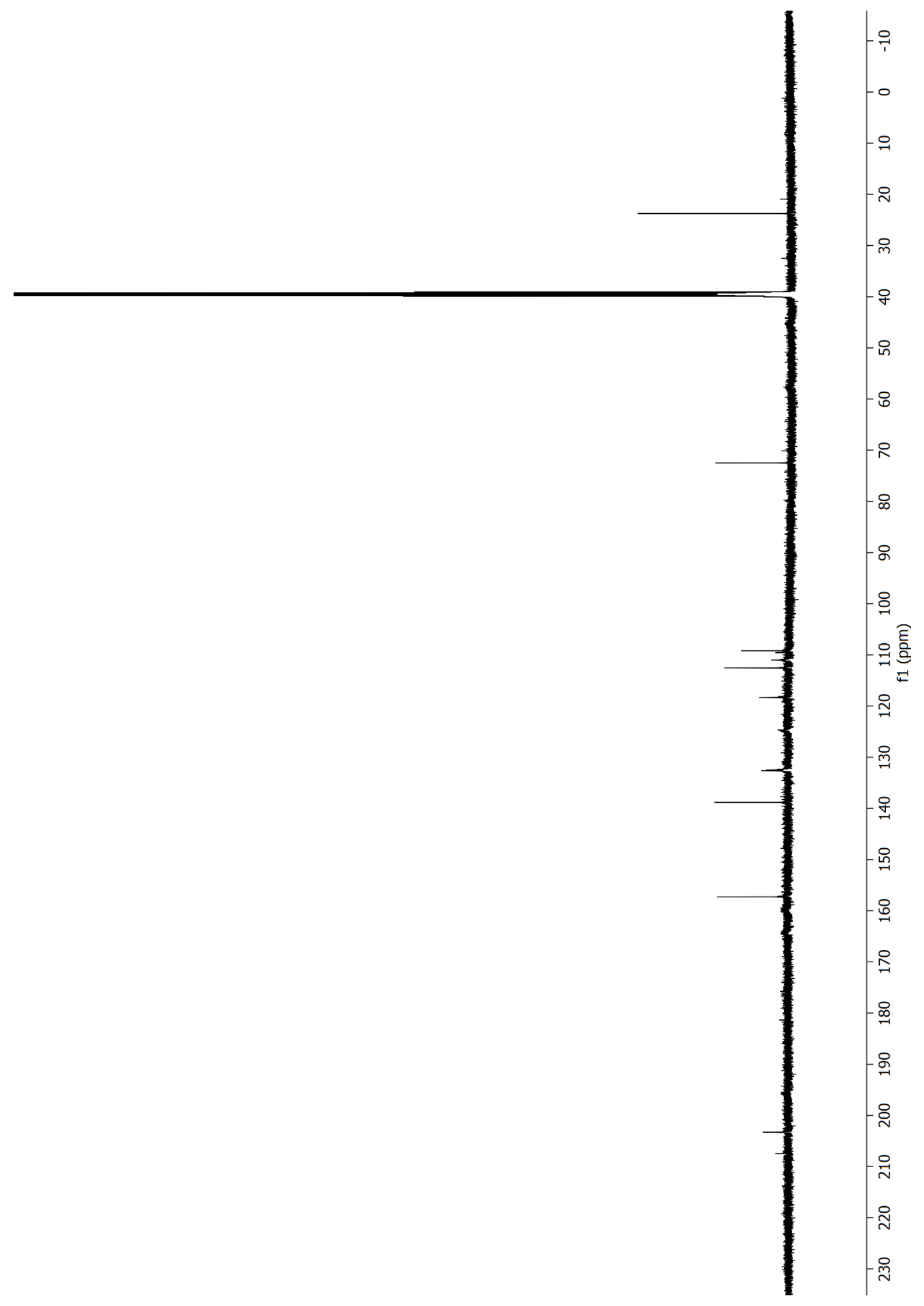


COSY spectrum of compound $2\left(600 \mathrm{MHz}, \mathrm{CDCl}_{3}\right)$

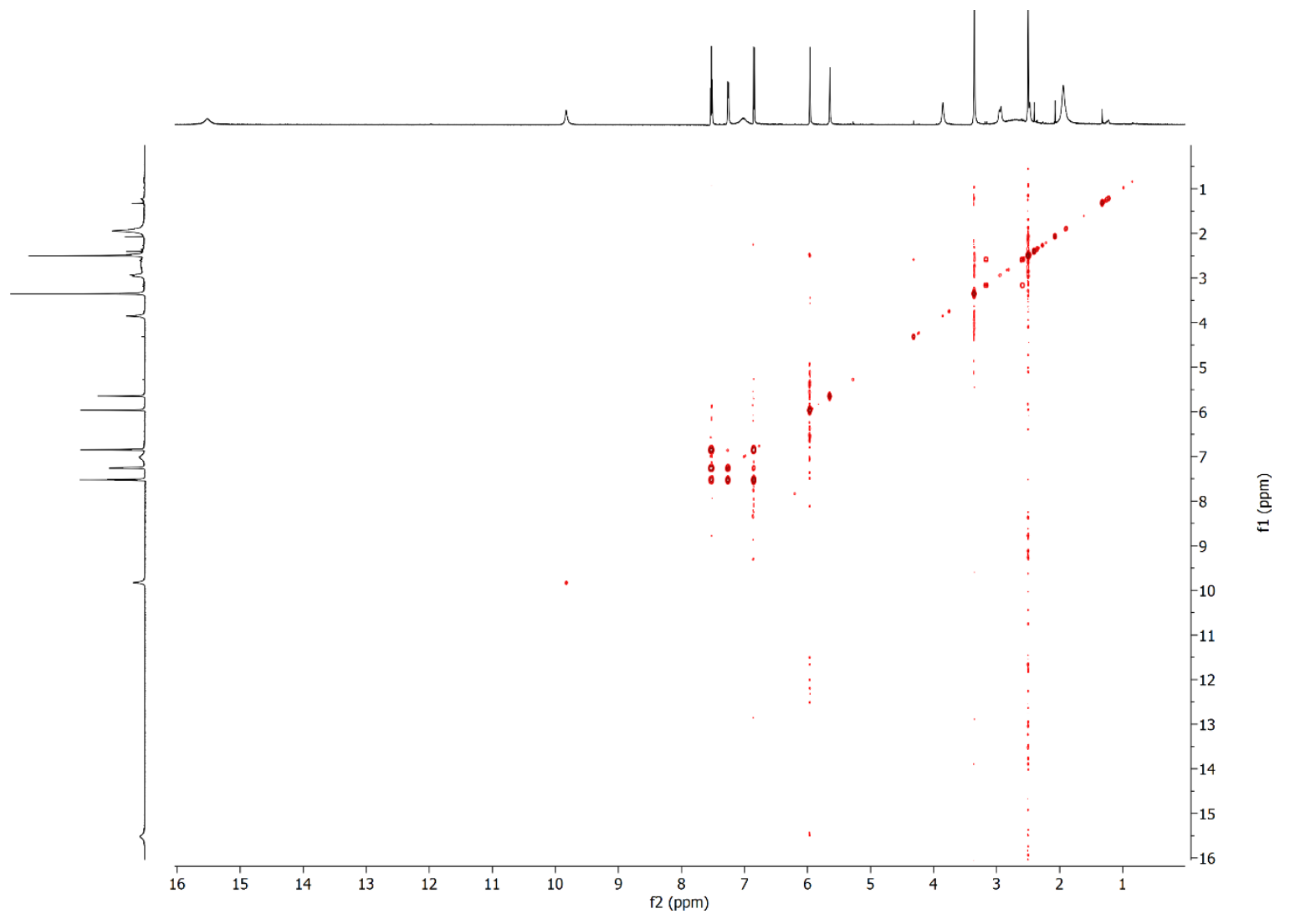

HSQC spectrum of compound $2\left(600 \mathrm{MHz}, \mathrm{CDCl}_{3}\right)$

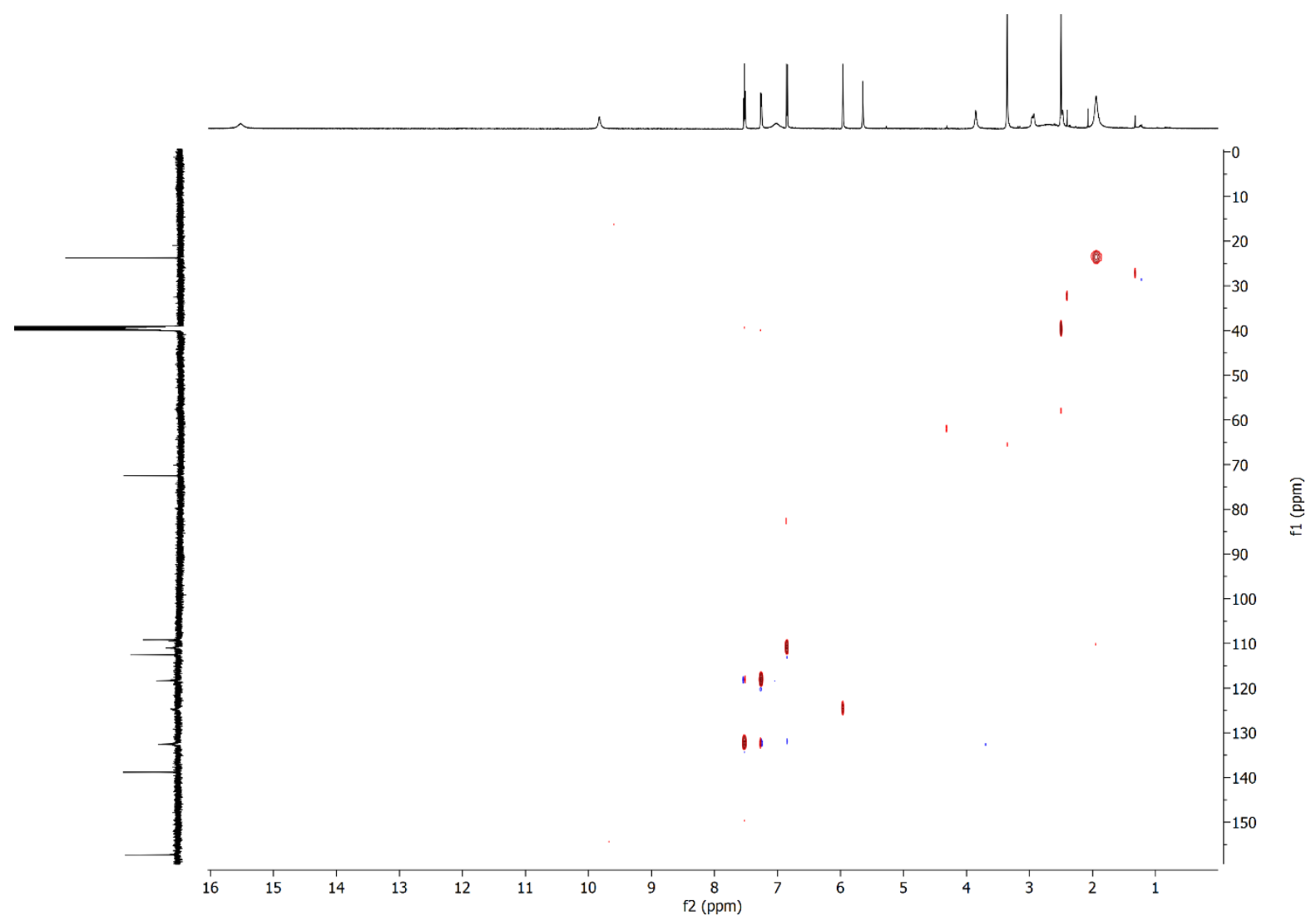


HMBC spectrum of compound $2\left(600 \mathrm{MHz}, \mathrm{CDCl}_{3}\right)$

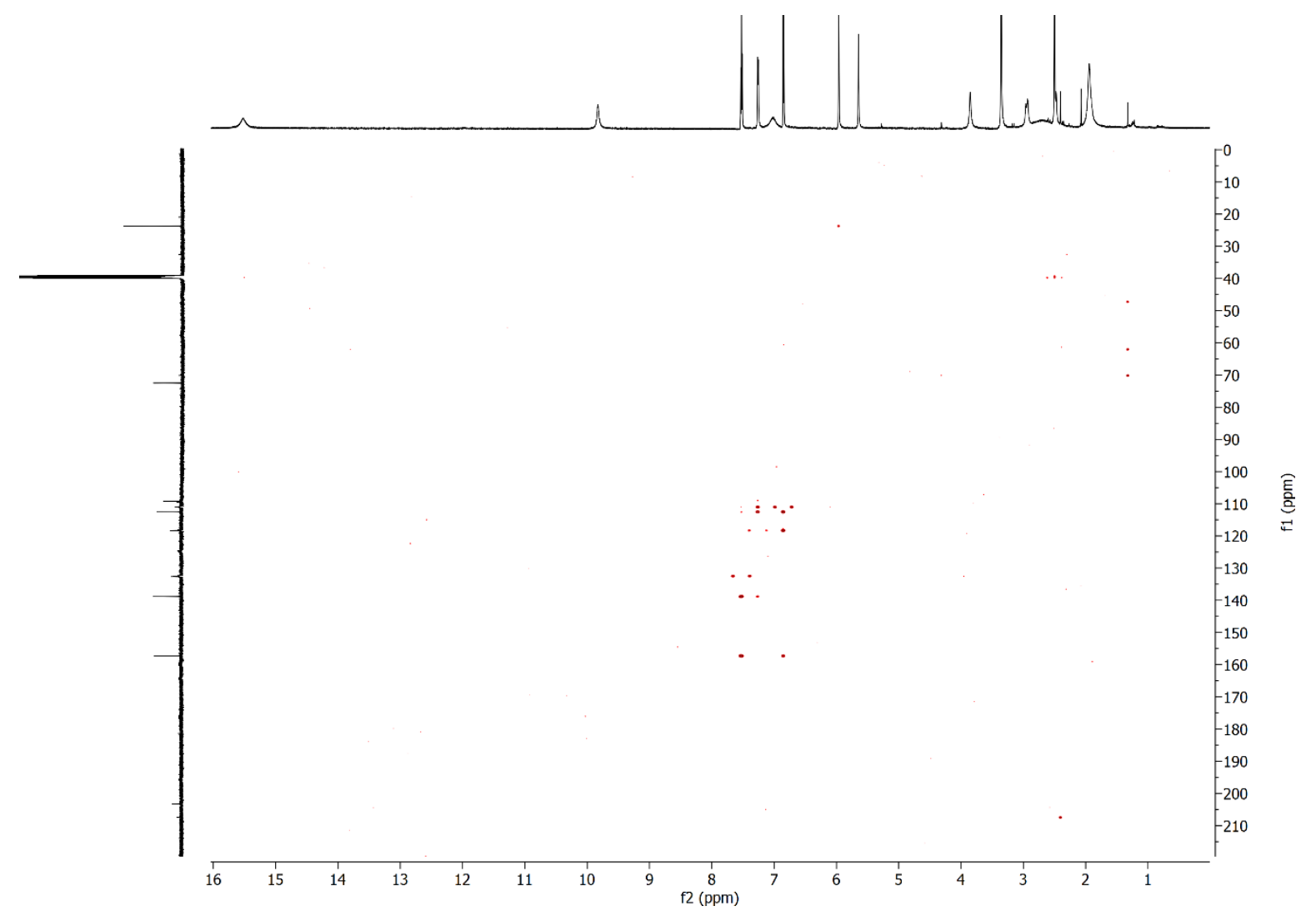


Appendix D. NMR spectra of compound 4

${ }^{1} \mathrm{H}$ spectrum of compound $4\left(600 \mathrm{MHz}, 1: 1 \mathrm{CDCl}_{3}: \mathrm{MeOD}\right)$

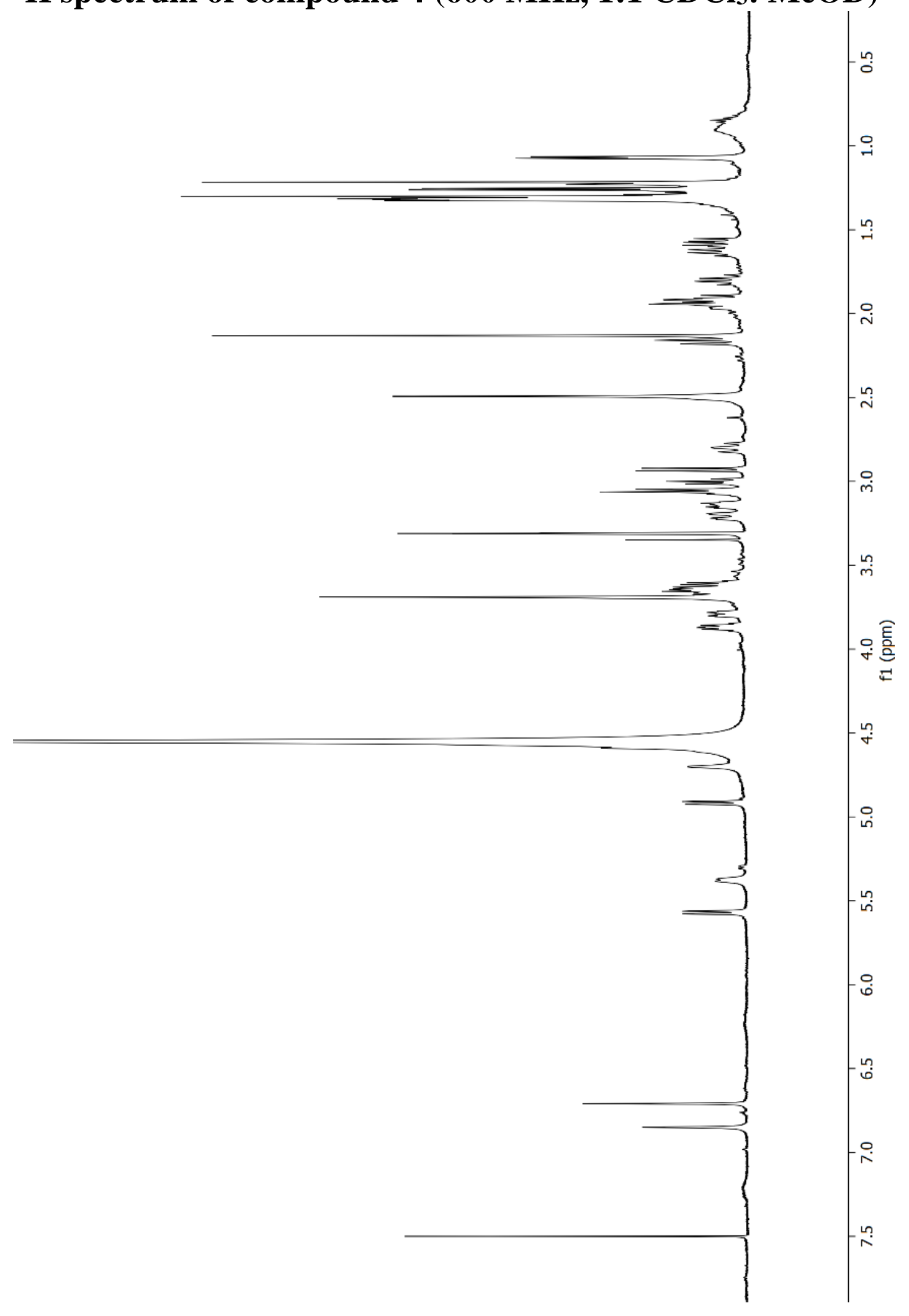




\section{${ }^{13} \mathrm{C}$ spectrum of compound $4\left(150 \mathrm{MHz}, 1: 1 \mathrm{CDCl}_{3}: \mathrm{MeOD}\right)$}

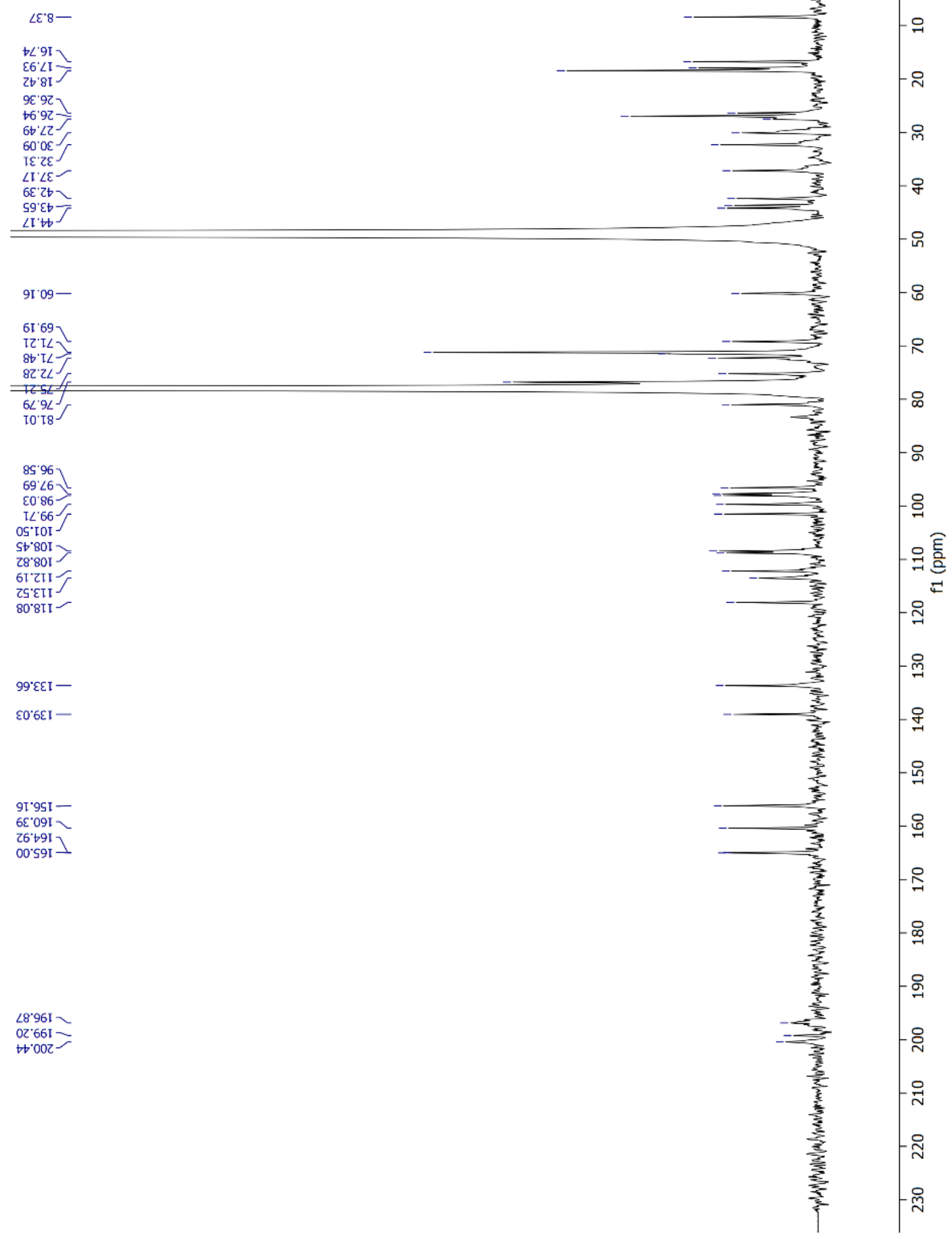


COSY spectrum of compound $4\left(600 \mathrm{MHz}, 1: 1 \mathrm{CDCl}_{3}: \mathrm{MeOD}\right)$

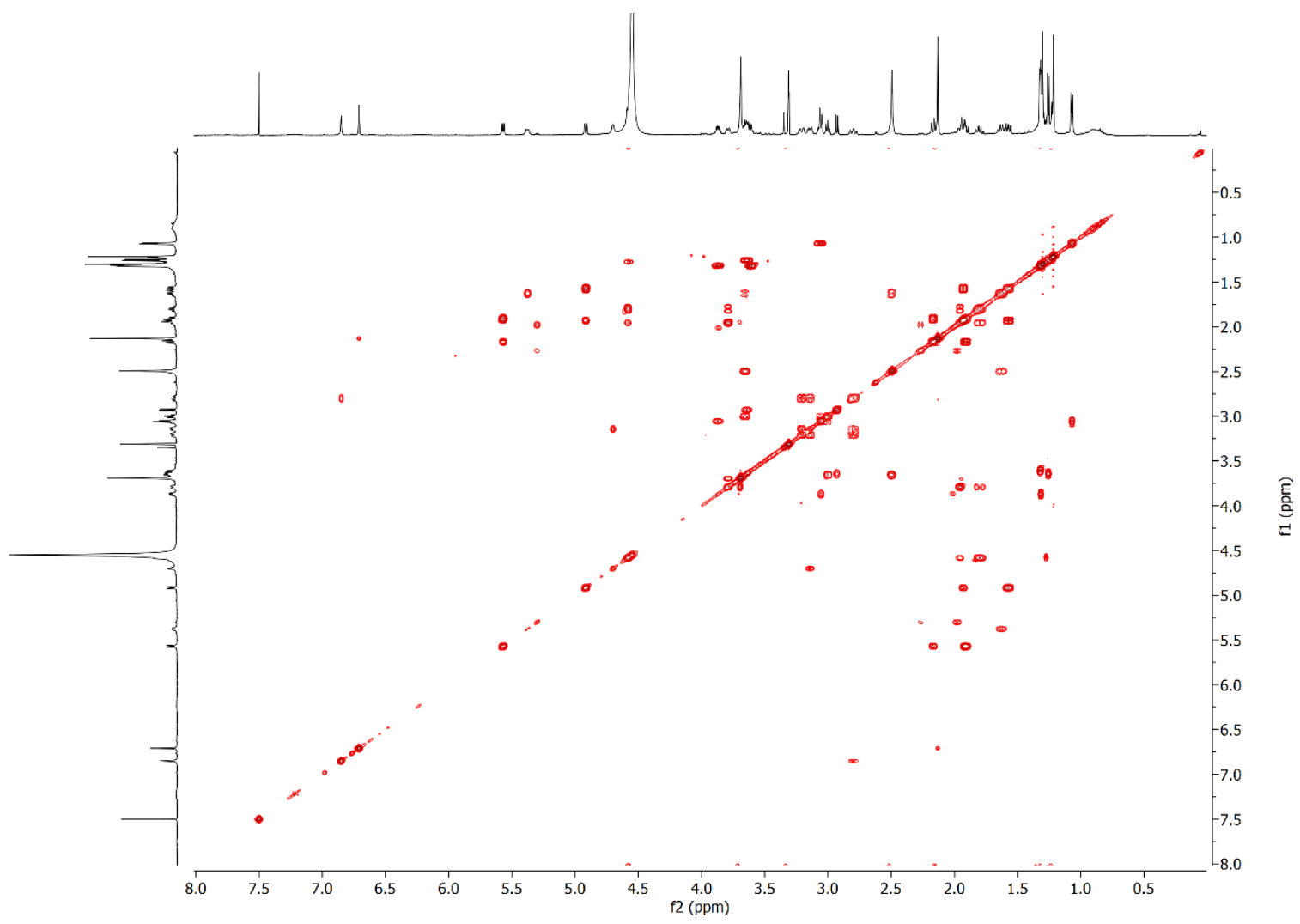

HSQC spectrum of compound $4\left(600 \mathrm{MHz}, 1: 1 \mathrm{CDCl}_{3}: \mathrm{MeOD}\right)$

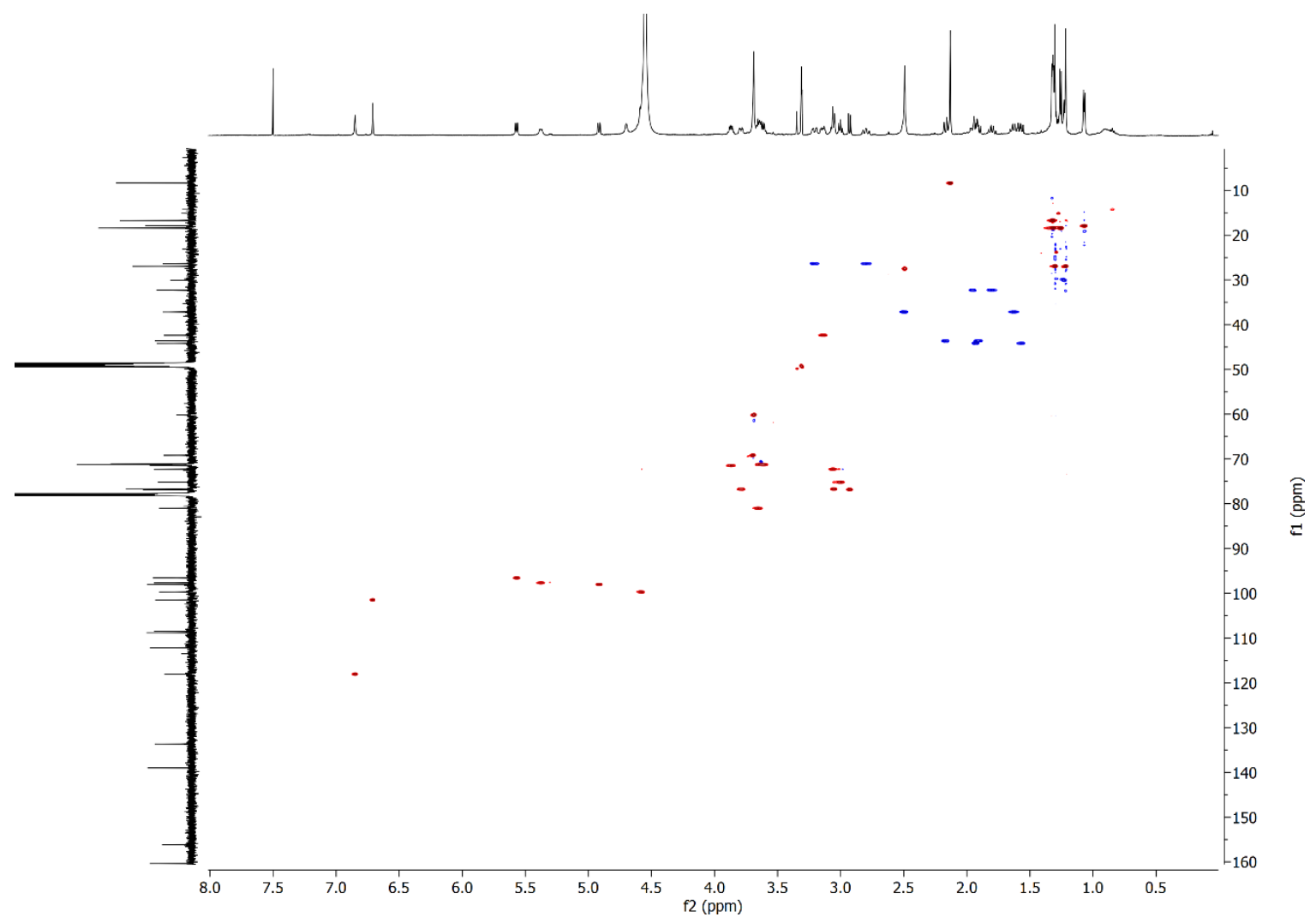


HMBC spectrum of compound $4\left(600 \mathrm{MHz}, 1: 1 \mathrm{CDCl}_{3}: \mathrm{MeOD}\right)$

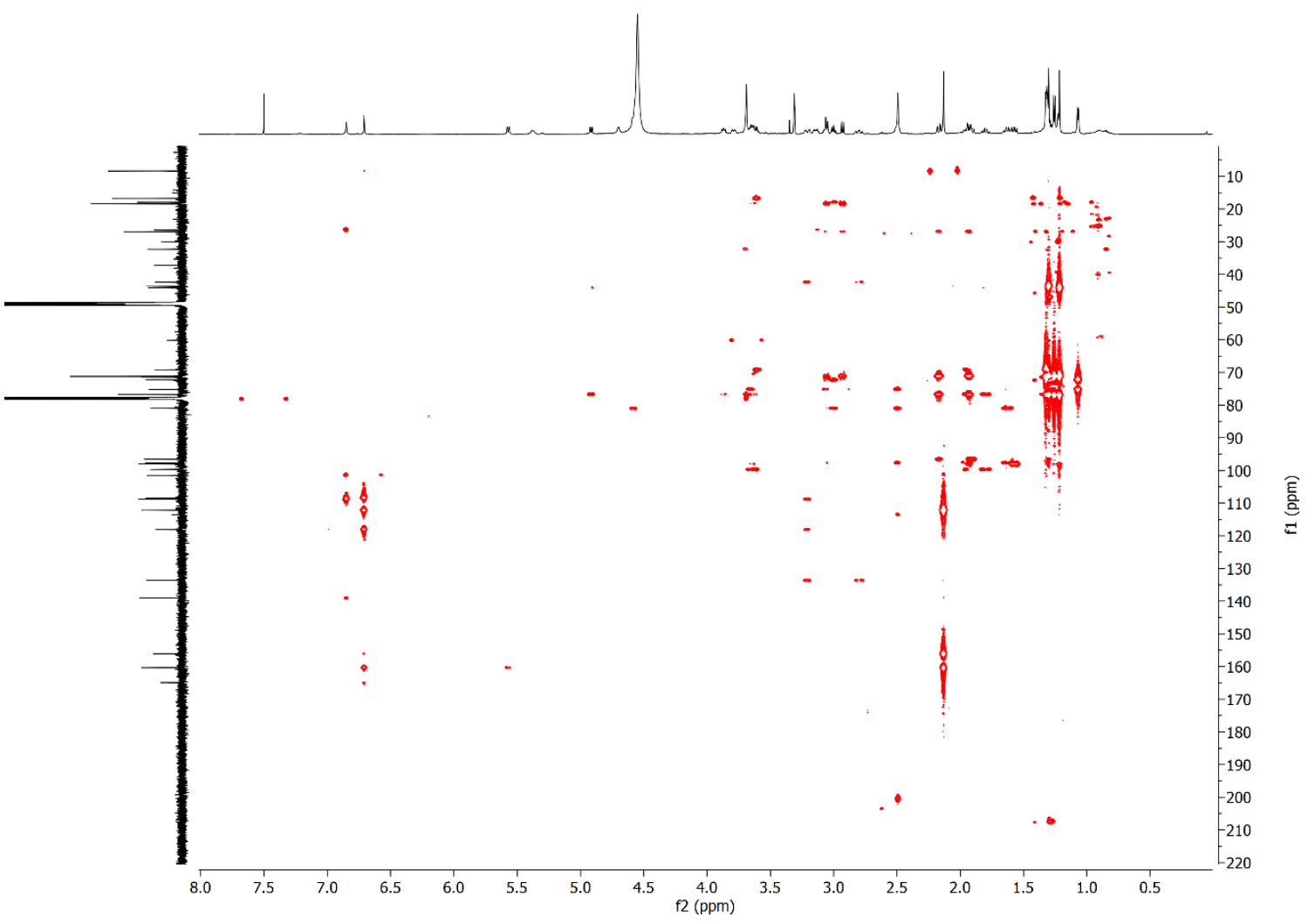

HSQC-TOCSY spectrum of compound $4\left(600 \mathrm{MHz}, 1: 1 \mathrm{CDCl}_{3}\right.$ : MeOD)

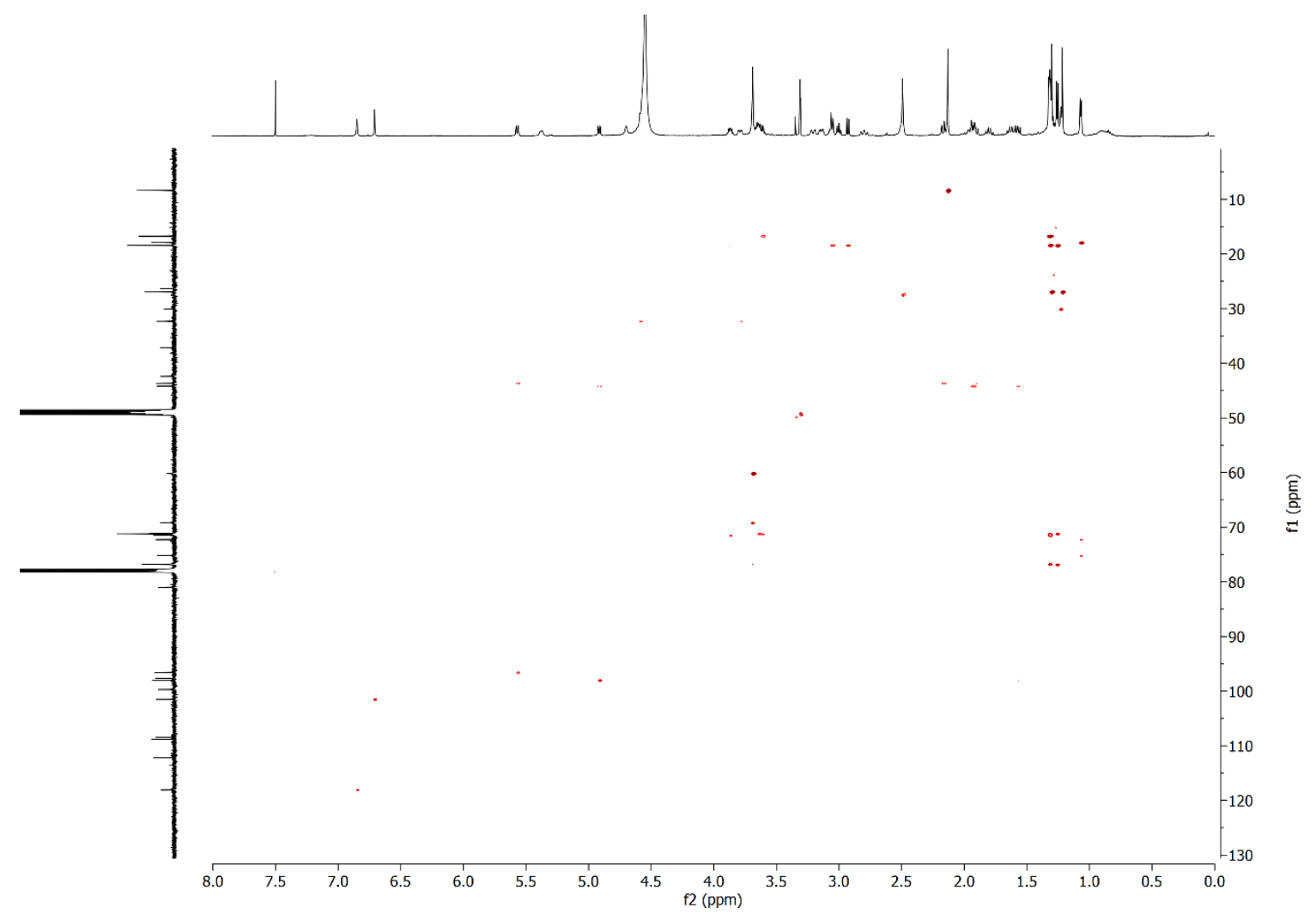


ROSEY spectrum of compound 4 (600 MHz, 1:1 CDCl 3 : MeOD)

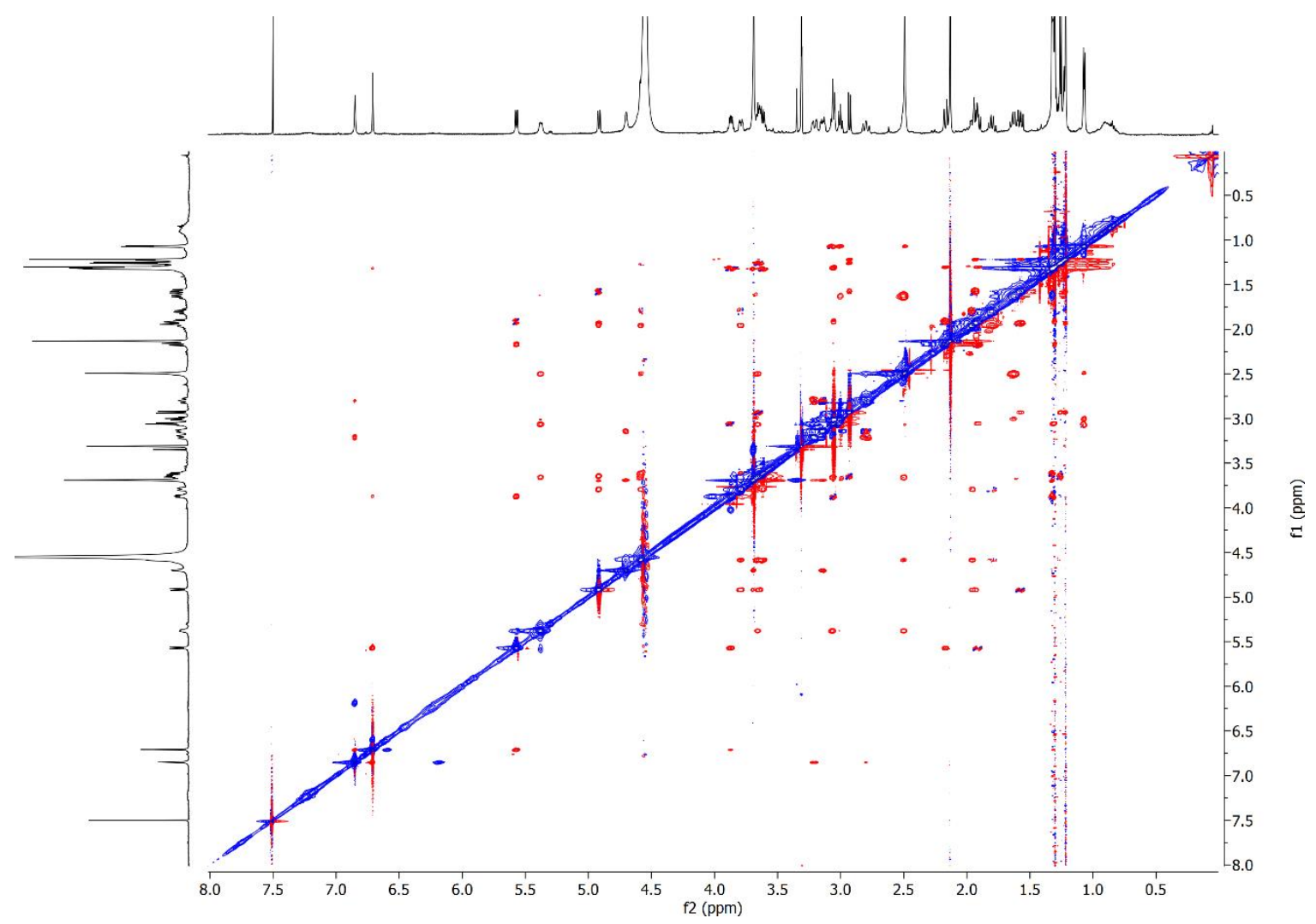




\section{Appendix E. Extended MS/MS spectra of compounds 4,}

\section{5 , and mithramycin}

\section{Mithramycin standard $\left([\mathrm{M}-\mathrm{H}]^{-}=1083.4647\right)$}

$20.0 \mathrm{eV}$

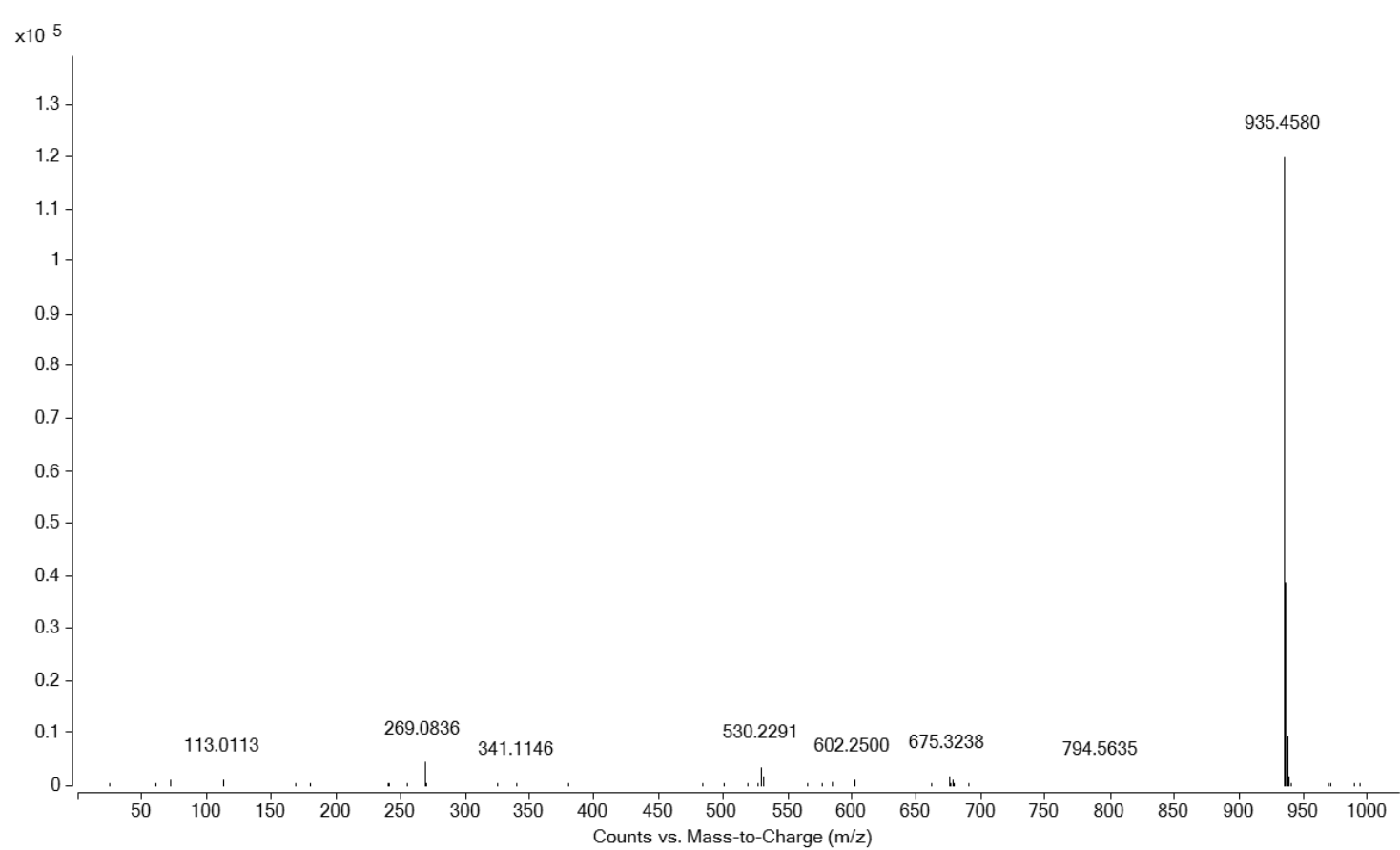

$60.0 \mathrm{eV}$

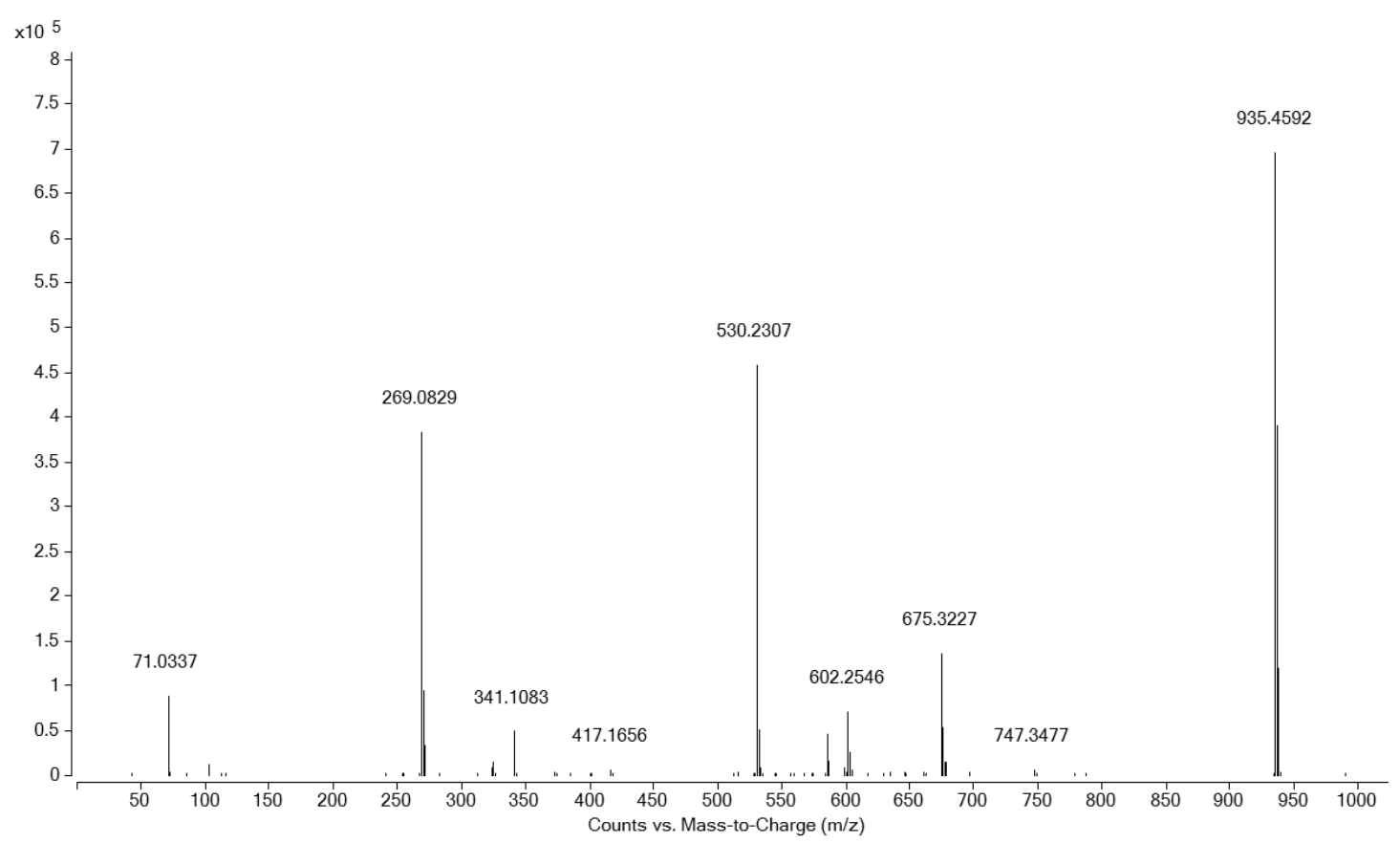


Compound $4\left([\mathrm{M}-\mathrm{H}]^{-}=\mathbf{9 7 5 . 3 8 4 8}\right)$

$20.0 \mathrm{eV}$

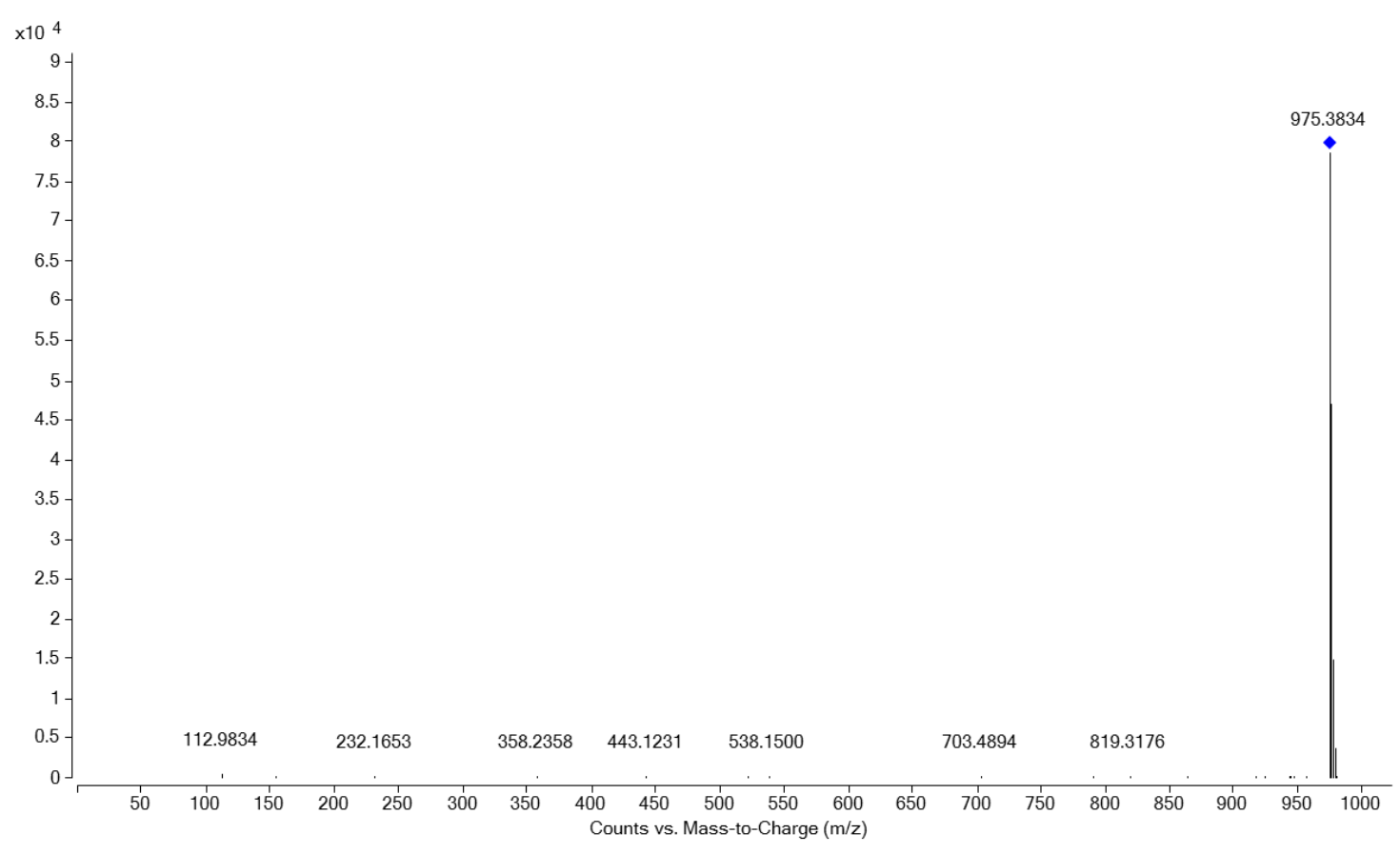

$60.0 \mathrm{eV}$

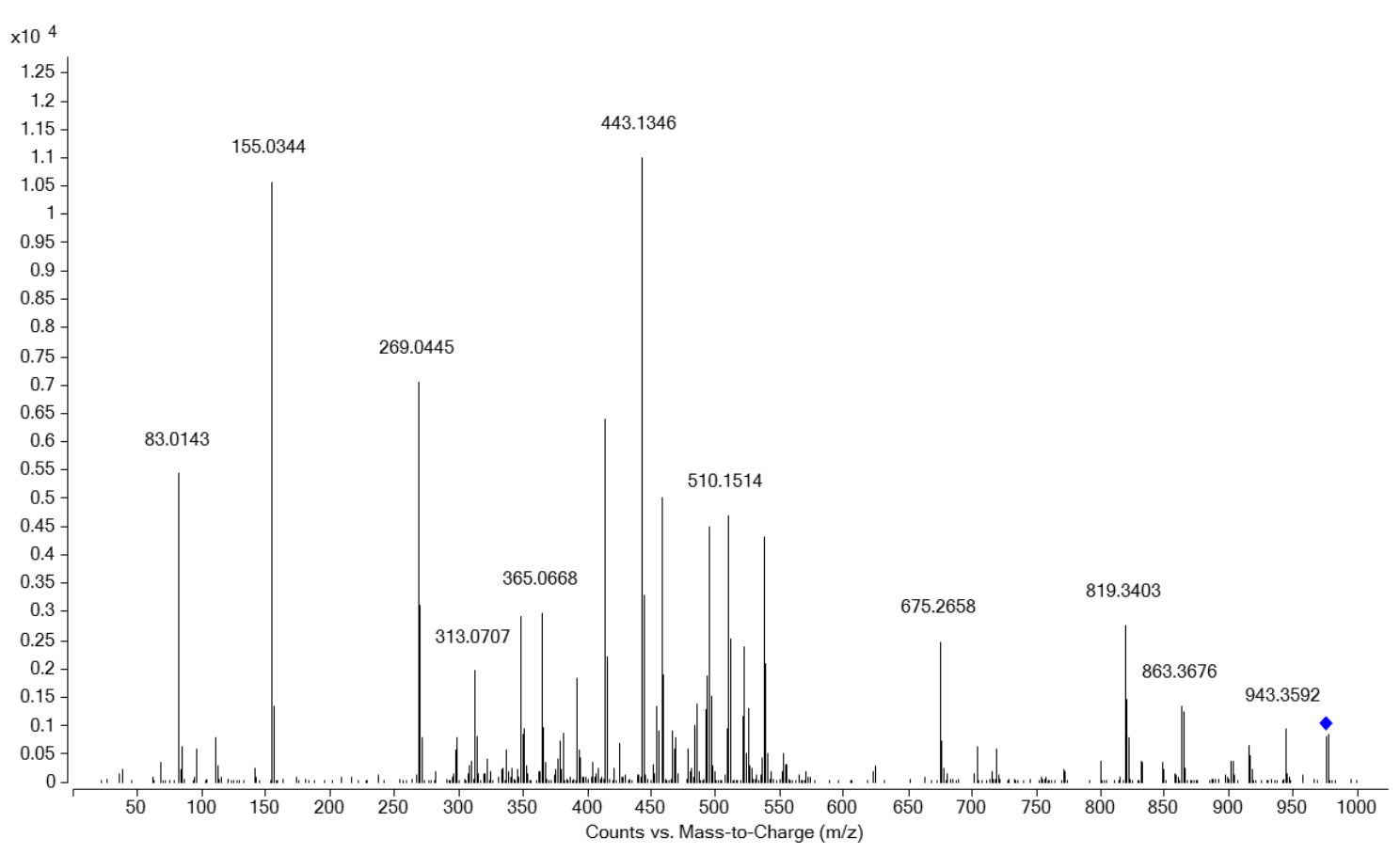




\section{Compound $5\left([\mathrm{M}-\mathrm{H}]^{-}=\mathbf{9 6 7 . 4 1 8 6}\right)$}

$20.0 \mathrm{eV}$

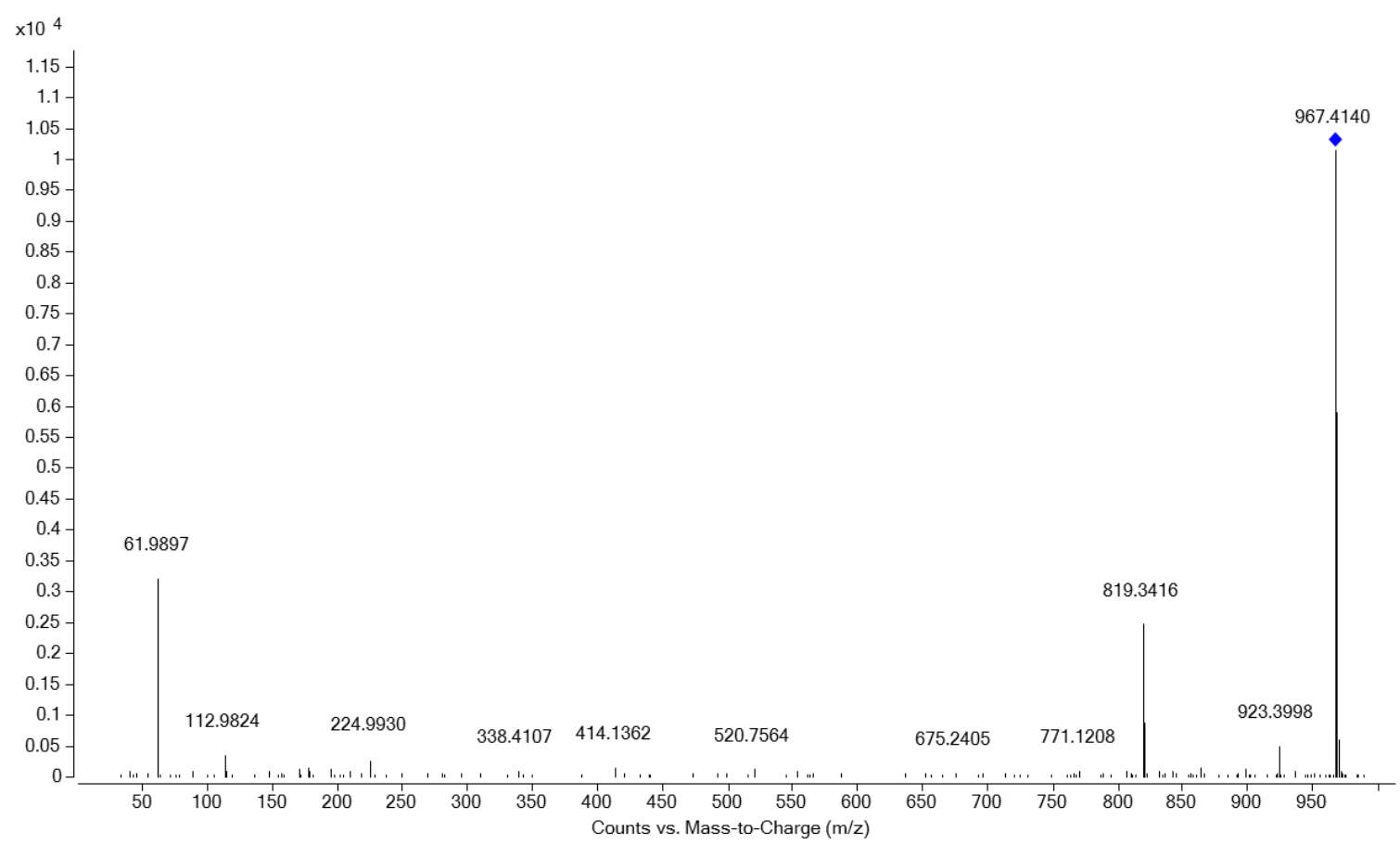

$60.0 \mathrm{eV}$

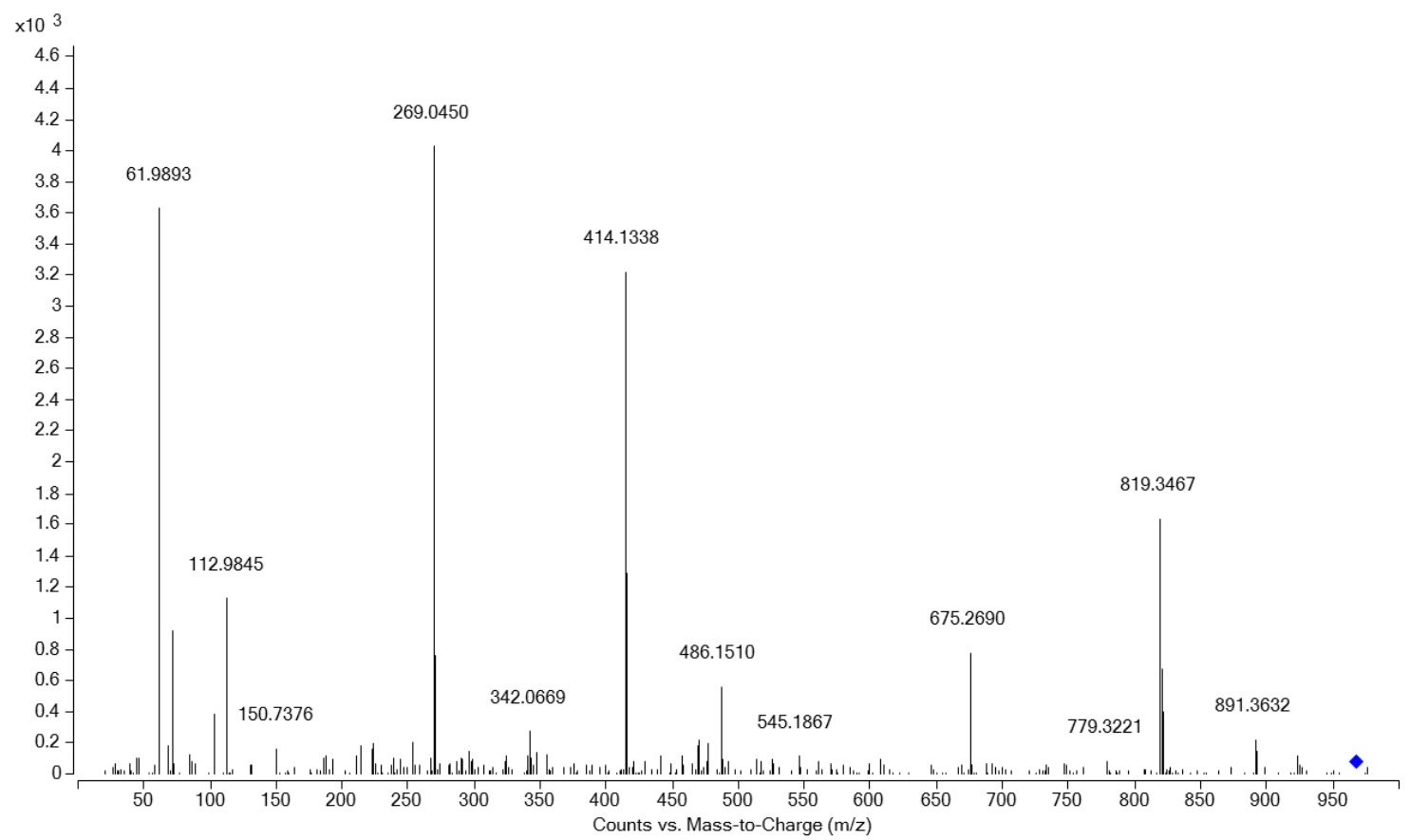


